\title{
20. CRETACEOUS CALCAREOUS NANNOFOSSIL BIOSTRATIGRAPHY OF SEDIMENTS RECOVERED FROM THE GALICIA MARGIN, ODP LEG 103 1
}

\author{
Joseph L. Applegate and James A. Bergen, Department of Geology, Florida State University, Tallahassee, Florida
}

\begin{abstract}
Ocean Drilling Program Leg 103 recovered Lower Cretaceous sediments from the Galicia margin off the coast of Iberia. The high diversity and abundance of assemblages makes this excellent material for the study of Early Cretaceous calcareous nannofossils.

With the exception of a hiatus between the upper Hauterivian and lower Barremian, nannofossil distributions form a continuous composite section from the lower Valanginian to lower Cenomanian sediments recovered at the four sites. The sedimentation history of this rifted continental margin is complex, and careful examination of the nannofossil content and lithology is necessary in order to obtain optimum biostratigraphic resolution.

The Lower Cretaceous sequence consists of a lower Valanginian calpionellid marlstone overlain by terrigenous sandstone turbidites deposited in the Valanginian and Hauterivian during initial rifting of this part of the margin. Interbedded calcareous marl and claystone microturbidites overlie the sandstone turbidites. Rifting processes culminated in the late Aptian-early Albian, resulting in the deposition of a calcareous, clastic turbidite sequence. The subsequent deposition of dark carbonaceous claystones (black shales) represents the beginning of seafloor spreading, as the margin continued to subside to depths near or below the CCD. The diversity, abundance, and preservation of nannofossils within these varied lithologies differ, and an attempt to distinguish between nearshore and open-marine assemblages is made. Genera used for this purpose include Nannoconus, Micrantholithus, Pickelhaube, and Lithraphidites.

In this study, six new species and one new subspecies are described and documented. Ranges of other species are extended, and an attempt is made to clarify existing, yet poorly understood, taxonomic concepts. A technique in which a single specimen is viewed with both light and scanning electron microscopes was used extensively to aid in this task. In addition, further subdivisions of the Sissingh (1977) zonation are suggested in order to increase biostratigraphic resolution.
\end{abstract}

\section{INTRODUCTION}

Leg 103 of the Ocean Drilling Program (ODP) drilled a total of 15 holes at five sites on the Galicia margin (Fig. 1). From four of the sites drilled, a nearly continuous, composite section ranging in age from early Valanginian to early Cenomanian is recognized based on the recovered nannofossil assemblages. The main goals of the cruise were to record and document the timing of rifting, subsidence, and sedimentation on the Galicia margin and to determine when rifting processes ceased and seafloor spreading began between Iberia and its conjugate margin, Newfoundland.

Sites 638,639 , and 641 were drilled on a tilted fault block in an attempt to recover pre-rift, syn-rift, and post-rift sediments (Fig. 2). These sites yielded a continuous, lithologically complex, composite section with abundant and well-preserved Lower Cretaceous nannofossils. The pre-rift sediments, which overlie continental basement, consist of crystalline limestones and dolomites, essentially devoid of nannofossils, that were deposited in shallow water in the Late Jurassic and earliest Cretaceous. As rifting began, terrigenous material was shed onto the rapidly subsiding margin in the form of turbidites. Episodic rifting continued up to the late Aptian/early Albian, when rifting ceased and seafloor spreading began. The corresponding change in sedimentation has been termed the break-up unconformity (Deep Sea Drilling Project (DSDP) Leg 47B; Sibuet, Ryan, et al., 1979 ) and is denoted by a change from shallow-water, clastic calcareous turbidites to deep-water dark claystones deposited near or below the carbonate compensation depth (CCD).

This study documents the occurrence, abundance, and preservation of Lower Cretaceous nannofossils recovered during ODP

\footnotetext{
${ }^{1}$ Boillot, G., Winterer, E. L., et al., 1988. Proc. ODP, Sci. Results, 103: College Station, TX (Ocean Drilling Program).
}

Leg 103. In addition, the relationship between lithology and nannofossil assemblages is discussed, and new taxa are described and documented. Six new species and one new subspecies are described from the Galicia margin Lower Cretaceous sequence. The ranges of numerous species, which are restricted in other studies because of ecologic, geographic, and/or preservational effects, have been extended. Several subdivisions of the Sissingh (1977) zonation are suggested and formally presented in this report.

Species considered in this report (Appendix A) are listed alphabetically by generic and specific epithets in Appendices B and $C$, respectively. Most of the bibliographic references for these taxa are given by Loeblich and Tappen $(1966,1968,1969$, 1970a, 1970b, 1971, 1973), Van Heck (1979a, 1979b, 1980a, 1980b, 1981a, 1981b, 1982a, 1982b, 1983), and Steinmetz (1984a, $1984 b, 1985,1986)$. Entries not found therein are given in the references.

\section{PREVIOUS LOWER CRETACEOUS NANNOFOSSIL STUDIES IN THE NORTH ATLANTIC BASIN}

Lower Cretaceous nannofossils were reported by Wind and Cepek from DSDP Site 397 (Sibuet, Ryan, et al., 1979) off the northwest coast of Africa. Moderately well-preserved Hauterivian(?) nannofossils were recovered and many new taxa were described from the site. However, problems with taxonomic concepts, preservation, and the limited section containing nannofossils made precise dating of the sediment recovered difficult.

Site 398 (Sibuet, Ryan, et al., 1979), south of Galicia Bank on the Vigo Seamount, recovered upper Hauterivian to Cenomanian sediments containing poorly preserved nannofossils (Blechschmidt, 1979), making this site a poor section for the study of Lower Cretaceous calcareous nannofossils.

Roth $(1978,1983)$ studied calcareous nannofossils from Lower Cretaceous sediment recovered at Sites 391 (DSDP Leg 44) and 


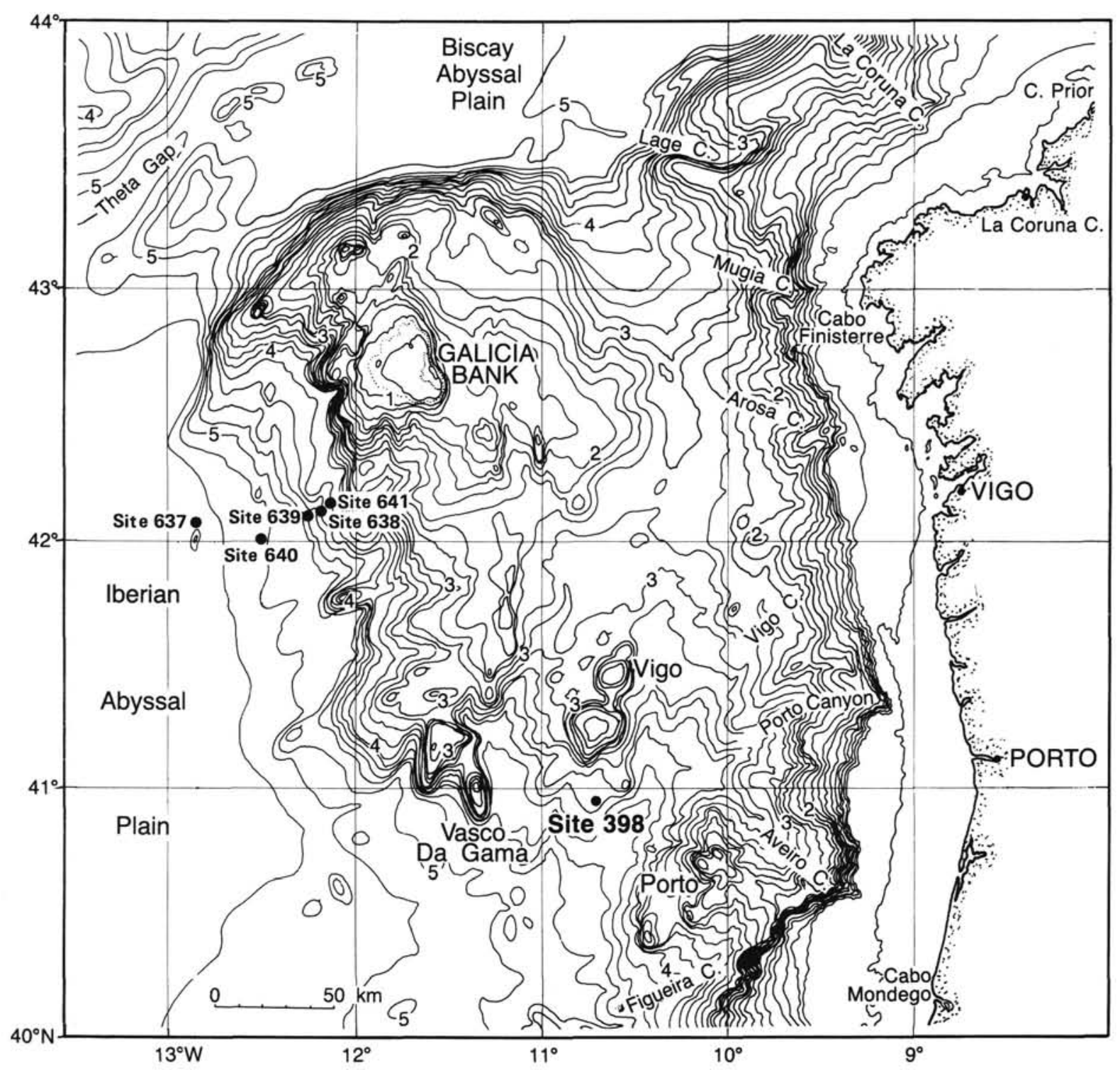

Figure 1. ODP Leg 103 site location map of the Galica margin. Bathymetry in kilometers.

534 (DSDP Leg 76) in the Blake-Bahama Basin, which provided him with the foundation for his coded number zonations.

Covington and Wise (1987) reported the nannofossil biostratigraphy of a Neocomian deep-sea fan complex at Site 603 off the eastern continental margin of North America. They noted variations in the composition of nannofossil assemblages with lithology at this site, where reworking by turbidites complicated the biostratigraphy. They attributed the nearshore nannofossil species present in the deep-sea environment to a process in which sediment is shed from within the oxygen-minimum zone along the upper slope to the outer shelf by turbidity currents. This phenomenon was widespread during the Neocomian in the North Atlantic-Tethyan realm.

\section{METHODS}

For the construction of range charts, standard smear slides were prepared from raw sediment using a method described by Hay (1970). Smear slides were then analyzed using a Zeiss Photomicroscope III under $1560 \times$ magnification. Abundances for each sample for both the total number of nannofossils and for individual species were estimated. Letters used on the range charts are keyed to the ${ }^{10} \log$ of the number of specimens likely to be observed in any one field of view. These and the corresponding logarithms are as follows:

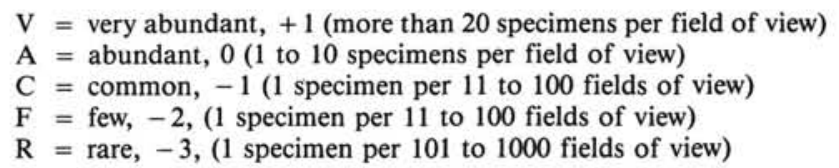

Reworked taxa are indicated on range charts by lowercase letters.

Nannofossil preservation in each sample is denoted as follows:

$\mathrm{G}=$ good (specimens show little or no dissolution and/or overgrowth)

$\mathrm{M}=$ moderate (specimens show some dissolution and/or overgrowth; identification of species not impaired)

$P=$ poor (specimens show significant dissolution and/or overgrowth; identification of species is impaired, but some still possible)

Selected samples containing well-preserved nannofossils were studied using a method devised by F. Wind (pers. comm., 1985) in which a single specimen is photographed with both light and scanning electron (SEM) microscopes. Wind used the method in several publications (Wise and Wind, 1977; Cepek and Wind, 1979) but never described the procedure. The method involves using a rubber stamp and rapidograph ink to 


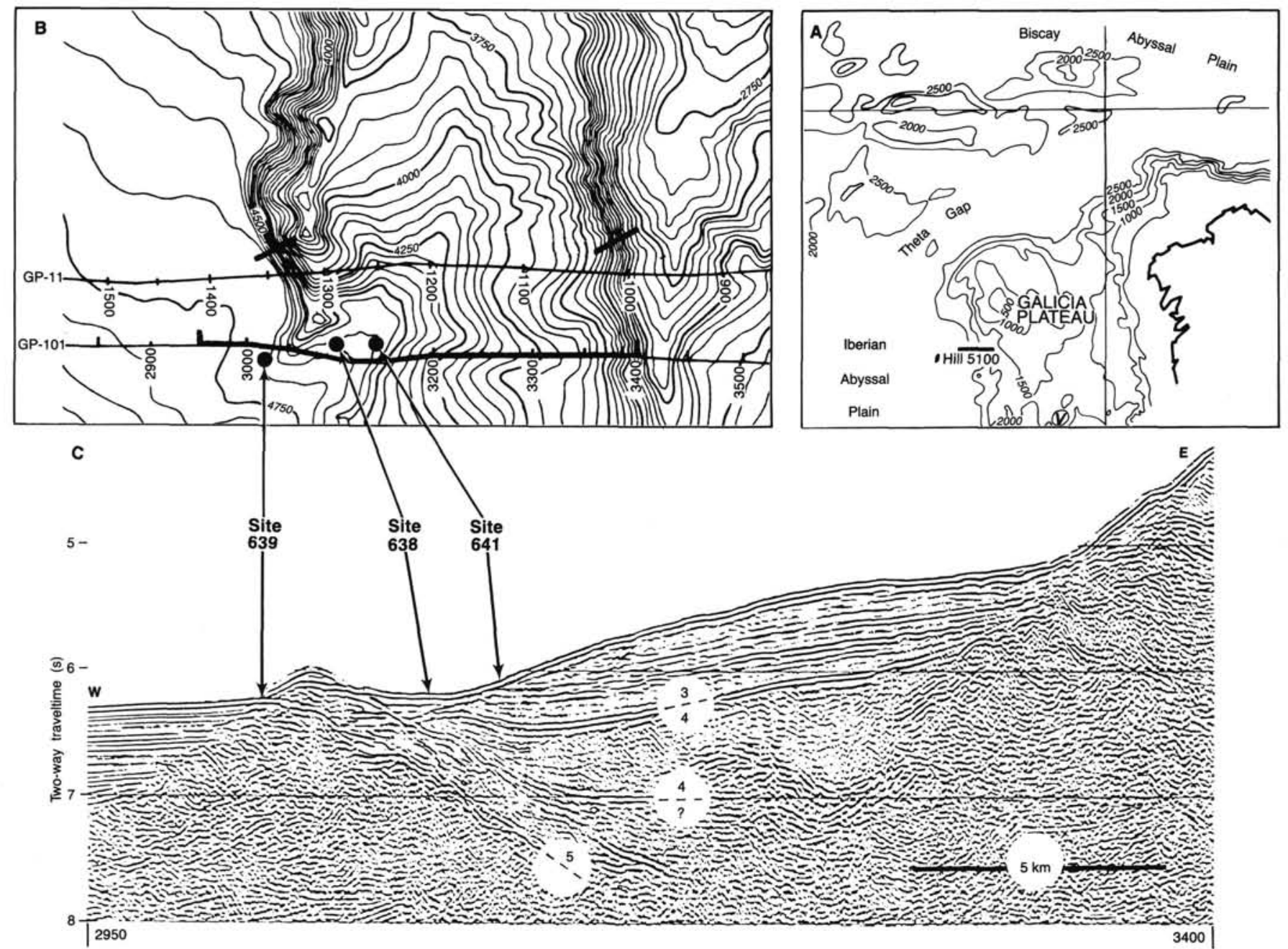

Figure 2. A. Galicia margin. B. Sea Beam map of the area near Sites 638, 639, and 641. C. Multichannel seismic profile GP-101, showing the location of Sites 638,639 , and $641.3=$ post-rift strata; 4 and $5=$ syn-rift strata. Bathymetry in meters. Courtesy of L. Montadert.

make a grid on a round SEM coverslip. A drop of solution containing concentrated nannofossils is placed on the coverslip and allowed to dry. The coverslip is then attached to a glass slide with the concentrate facing up, and immersion oil is placed directly onto the concentrate. Specimens are photographed under the light microscope, and their location with respect to letters and other objects on the grid is recorded. The coverslip is submerged in a xylene bath three times and washed in isopropyl alcohol until all oil is removed and the sample on the coverslip appears dry, which only takes a few minutes. The coverslip is glued to an SEM stub and coated with a gold palladium alloy. The sample is then viewed under the SEM, and the specimens are relocated and photographed. This technique was used extensively in this study to document new species and to add more information for resolving existing taxonomic problems of several Lower Cretaceous nannofossils.

\section{ZONATION AND SPECIES CONSIDERED}

Past studies of Lower to middle Cretaceous nannofossils have produced many zonations. Different biogeographic settings, poor preservation of assemblages, and a general lack of studied Lower Cretaceous sections are several reasons why there is no widely accepted zonation. The majority of Lower Cretaceous zonations are based on Thierstein (1971, 1973, 1976), who studied stratotype and paratype sections in Europe and correlated the nannofossil biostratigraphy to ammonite, calpionellid, and foraminiferal biostratigraphy in the framework of the Lower Cretaceous Stages. Sissingh (1977) first applied a coded numbering system to the Cretaceous, modifying many of the existing Lower to middle Cretaceous zonations of Thierstein (1971, 1973) and Manivit et al. (1977). Perch-Nielsen (1979, 1985) further refined the zonation of Sissingh (1977) by correlating a significant number of nannofossil events to his numbercoded (CC) zones. This zonation is closely followed in this report. It is also compared with the number-coded (NC) zonation of Roth $(1978,1983)$, based on the Lower Cretaceous of the western North Atlantic. A summary chart of both zonations along with modifications is shown in Figure 3 . The following is a summary of the zonation scheme used in this report.

Retecapsa angustiforata Zone (CC2)

Definition. Interval from the first occurrence of Retecapsa angustiforata to the first occurrence of Calcicalathina oblongata.

Authors. Thierstein, 1971.

Age. Late Berriasian to early Valanginian.

\section{Tubodiscus verenae Subzone (CC3a) of the Calcicalathina oblongata Zone (CC3)}

Definition. Interval from the first occurrence of $C$. oblongata to the first occurrence of Eiffellithus windii.

Authors. CC3: Thierstein (1971), modified by Sissingh (1977) and Perch-Nielsen (1979). CC3a: this paper.

Age. Early Valanginian.

Remarks. The subdivision of $\mathrm{CC} 3$ is suggested here based on the first occurrence of $E$. windii $\mathrm{n}$. sp. Although described here as new, it is illustrated as Eiffellithus sp. in earlier reports (see Appendix A). Al- 


\begin{tabular}{|c|c|c|c|c|c|c|}
\hline \multirow[b]{2}{*}{ Stage } & \multirow{2}{*}{$\begin{array}{l}\text { Age (m.y.) } \\
\text { Van Hinte } \\
(1976)\end{array}$} & \multicolumn{2}{|c|}{ Zone } & \multicolumn{2}{|r|}{ Subzone } & \multirow[t]{2}{*}{ Datum level } \\
\hline & & $\begin{array}{c}\text { Roth (1978, } \\
\text { 1983) }\end{array}$ & $\begin{array}{l}\text { Sissingh (1977), } \\
\text { modified }\end{array}$ & & & \\
\hline \multirow{3}{*}{$\begin{array}{c}\text { early } \\
\text { Cenomanian }\end{array}$} & & \multirow{2}{*}{$\mathrm{NCl1}$} & \multirow{2}{*}{ CC10 Microrhabdulus decoratus } & $\mathrm{CCl} 10 \mathrm{~b}$ & Gartnerago oblipuum & \multirow{2}{*}{$\frac{\text { FAD Quadrum gartneri }}{\text { LAD Microstaurus chiastius }}$} \\
\hline & & & & $\mathrm{CC} 10 \mathrm{a}$ & Microstaurus chiastius & \\
\hline & $100-$ & \multirow{2}{*}{$\mathrm{NC10}$} & \multirow{2}{*}{ CC9 Eiffellithus turriseiffeli } & $\mathrm{CC} 9 \mathrm{~b}$ & Prediscophaera spinosa & \multirow{2}{*}{$\begin{array}{l}\text { FAD Lithraphidites acutus } \\
\text { LAD Hayesites albiensis }\end{array}$} \\
\hline \multirow{3}{*}{ Albian } & & & & $\mathrm{CC} 9 \mathrm{a}$ & Hayesites albiensis & \\
\hline & & NC9 & CC8 Prediscosnhaera columnata & $\mathrm{CC} 8 \mathrm{~b}$ & Tranolithus phacelosus & FAD Eiffellithus turriseiffeli \\
\hline & & NC8 & & $\mathrm{CC} 8 \mathrm{a}$ & Braarudosphaera africana & FAD Tranolithus phacelosus \\
\hline \multirow{2}{*}{ Aptian } & & NC7 & \multirow{2}{*}{ CC7 Chiastozygus litterarius } & $\mathrm{CC} 7 \mathrm{~b}$ & Rhagodiscus angustus & FAD Prediscosphaera columnata \\
\hline & & NC6 & & $\mathrm{CC7a}$ & Hayesites irregularis & FAD Eprolithus floralis \\
\hline \multirow{2}{*}{ Barremian } & \multirow{4}{*}{121} & NC5b & CC6 Micrantholithus hoschulzii & & & FAD Hayesites irregularis \\
\hline & & NC5a & CC5 Lithraphidites bollii & & & LAD Calcicalathina oblongata \\
\hline \multirow{2}{*}{ Hauterivian } & & $\mathrm{NC} 4 \mathrm{~b}$ & \multirow{2}{*}{ CC4 Cretarhabdus loriei } & $\mathrm{CC} 4 \mathrm{~b}$ & Speetonia colligata & LAD Speetonia colligata \\
\hline & & $\mathrm{NC} 4 \mathrm{a}$ & & $\mathrm{CC} 4 \mathrm{a}$ & Eiffellithus striatus & FAD Lithraphidites bollii \\
\hline \multirow{2}{*}{ Valanginian } & 120 & \multirow{2}{*}{$\mathrm{NC} 3$} & \multirow{2}{*}{ CC3 Calcicalathina oblongata } & $\mathrm{CC} 3 \mathrm{~b}$ & Eiffellithus windii & FAD Eiffellithus striatus \\
\hline & & & & $\mathrm{CC} 3 \mathrm{a}$ & Tubodiscus verenae & FAD Eiffellithus windii \\
\hline \multirow{2}{*}{ Berriasian } & . & $\mathrm{NC2}$ & CC2 Retacapsa angustiforata & & & FAD Calcicalathina oblongata \\
\hline & & $\mathrm{NCl}$ & CC1 Nannoconus steinmannii & & & FAD Retacapsa angustiforata \\
\hline
\end{tabular}

Figure 3. Summary figure of zonations considered and used in this study.

though rare to few in abundance in the study material, $E$. windii is taxonomically distinct and stratigraphically consistent in the early part of its range, making it a useful datum.

\section{Eiffellithus windii Subzone (CC3b) of the Calcicalathina oblongata Zone (CC3)}

Definition. Interval from the first occurrence of $E$. windii to the first occurrence of Eiffellithus striatus.

Authors. CC3: Thierstein (1971), modified by Sissingh (1977) and Perch-Nielsen (1979). CC3b: this paper.

Age. CC3b: late Valanginian.

Remarks. The $C$. oblongata Zone was originally defined by Thierstein (1971) and extended from the first occurrence of $C$. oblongata to the first occurrence of Lithraphidites bollii. Sissingh (1977) later modified the top of the zone to coincide with the first occurrence of Cretarhabdus loriei. Perch-Nielsen (1979) suggested the substitution of the first occurrence of $E$. striatus for the first occurrence of $C$. loriei in the Boreal region, a datum followed in this study.

As previously mentioned, Sissingh (1977) used the first occurrence of C. loriei (Gartner) to mark the top of this zone. However, some workers restrict this species concept to forms that occur only in Aptian and younger strata and, thus, do not recognize this species in older sediments. The top of Roth's (1978) NC3 Zone at the Valanginian/Hauterivian boundary is defined by the last occurrence of $T$. verenae and Diadorhombus rectus. In the study material, $T$. verenae ranges well up into the Hauterivian above the first occurrence of $L$. bollii, whereas $D$. rectus ranges into the upper Barremian above the last occurrence of $C . o b$ longata, L. bollii, and Speetonia colligata. Therefore, the last occurrence of $T$. verenae and $D$. rectus appear diachronous and cannot be used to define the Valanginian/Hauterivian boundary. The last occurrence of an additional form used by Roth (1983), Cyclagelosphaera deflandrei, seems to be controlled by preservation and is not considered trustworthy (Covington and Wise, 1987). The rare but consistent occurrence of the distinctive form $E$. striatus makes it a useful datum for the top of $\mathrm{CC} 3$ in this study.

\section{Eiffellithus striatus Subzone (CC4a) of the Cretarhabdus loriei Zone (CC4)}

Definition. CC4a: interval from the first occurrence of $E$. striatus to the first occurrence of $L$. bollii.

Authors. CC4: Sissingh (1977), modified by Perch-Nielsen (1979). $\mathrm{CC} 4 \mathrm{a}:$ this paper.
Age. CC4a: early Hauterivian.

Remarks. In this region, the occurrence of certain nannofossil species such as $L$. bollii and $C$. oblongata suggest a Tethyan or tropical environment. However, other forms previously reported only from Boreal regions, such as Nannoconus abundans and E. striatus, are also present, suggesting that the Galicia margin occupied a region intermediate between the two realms.

\section{Speetonia colligata (CC4b) Subzone of the Cretarhabdus loriei Zone (CC4)}

Definition. Interval from the first occurrence of $L$. bollii to the last occurrence of $S$. colligata.

Authors. CC4: Sissingh (1977) and Perch-Nielsen (1979). CC4b: this paper.

Age. CC4b: late early Hauterivian to late Hauterivian.

\section{Lithraphidites bollii Zone (CC5)}

Definition. Interval from the last occurrence of $S$. colligata to the last occurrence of $C$. oblongata.

Authors. Thierstein (1971), emended by Sissingh (1977).

Age. Latest Hauterivian to early Barremian.

\section{Micrantholithus hoschulzii Zone (CC6)}

Definition. Interval from the last occurrence of $C$. oblongata to the first occurrence of Hayesites irregularis.

Authors. Thierstein (1971), emended by Thierstein (1973) and Sissingh (1977).

Age. Late Barremian.

Remarks. Thierstein (1976) noted that the first occurrence of $H$. irregularis is right before the first occurrence of Chiastozygus litterarius (or Chiastozygus platyrhethus in Perch-Nielsen, 1979). However, the first occurrence of $C$. literarius has been clearly shown in this study and by other authors to extend into the Hauterivian, rendering its first occurrence useless as a Barremian-Aptian datum. The last occurrence of Nannoconus steinmannii/colomii, which Thierstein $(1971,1973)$ placed at the top of CC6, extends into the lower Aptian and cannot be used to recognize the Barremian/Aptian boundary. Therefore, the first occurrence of the distinctive form $H$. irregularis (see Appendix A) is used in this study to mark the nannofossil Barremian/Aptian boundary. 
Hayesites irregularis Subzone (CC7a) of the Chiastozygus litterarius Zone (CC7)

Definition. Interval from the first occurrence of $H$. irregularis to the first occurrence of Eprolithus floralis.

Authors. CC7a: Thierstein (1971), emended by Manivit et al. (1977). Age. CC7a: early Aptian.

Remarks. Thierstein (1971) suggested that the first occurrence of Rhagodiscus angustus coincides with that of $E$. floralis, but it is likely that $R$. angustus has its first occurrence higher than $E$. floralis. The last occurrences of Micrantholithus hoschulzii/obtusus, N. steinmannii, and Conusphaera mexicana all occur near the top of $\mathrm{CC} 7 \mathrm{a}$ but are difficult to use where reworking exists.

\section{Rhagodiscus angustus Subzone (CC7b) of the Chiastozygus litterarius Zone (CC7)}

Definition. First occurrence of $E$. floralis to the first occurrence of Prediscosphaera columnata.

Authors. CC7b: Thierstein (1971), emended by Manivit et al. (1977).

Age. Late Aptian to earliest Albian.

Remarks. This subzone corresponds to Thierstein's (1971) Parhabdolithus angustus Zone.

\section{Braarudosphaera africana Subzone (CC8a) of the Prediscosphaera columnata Zone (CC8)}

Definition. Interval from the first occurrence of $P$. columnata to the first occurrence of Tranolithus phacelosus (= Tranolithus exiguus $=$ Tranolithus orionatus).

Authors. CC8: Thierstein (1971), emended by Manivit et al. (1977). CC8a: suggested by Perch-Nielsen (1979), formally presented in this paper.

Age. CC8a: early Albian.

Remarks. Perch-Nielsen (1979) first suggested the subdivision of CC 8 into two subzones by using the first occurrences of the co-markers T. phacelosus and Corollithion signum, and the genus Cribrosphaerella. The subdivision is formally presented here.

\section{Tranolithus phacelosus Subzone (CC8b) of the Prediscosphaera columnata Zone (CC8)}

Definition. Interval from the first occurrence of $T$. phacelosus to the first occurrence of Eiffellithus turriseiffeli.

Authors. CC8: Thierstein (1971), emended by Manivit et al. (1977). CC8b: suggested by Perch-Nielsen (1979), formally presented in this paper.

Age. Late Albian.

Hayesites albiensis Subzone (CC9a) and Prediscosphaera spinosa Subzone (CC9b) of the Eiffellithus turriseiffeli Zone (CC9)

Definition. CC9a: the interval from the first occurrence of $E$. turriseiffeli to the last occurrence of Hayesites albiensis. CC9b: the interval from the last occurrence of $H$. albiensis to the first occurrence of Lithraphidites acutus.

Age: CC9a: late Albian. CC9b: latest Albian to early Cenomanian.

Remarks. The subzones of $\mathrm{CC} 9$ are basically those of Manivit et al. (1977), which have been correlated to the number-coded scheme of Sissingh (1977).

Microstaurus chiastius (CC10a) of the Microrhabdulus decoratus Zone ( $\mathrm{CC10})$

Definition: Interval from the first occurrence of $L$. acutus to the last occurrence of Microstaurus chiastius.

Age. Late early Cenomanian.

Gartherago obliquum Subzone (CC10b) of the Microrhabdulus decoratus Zone (CC10)

Definition. Interval from the last occurrence of $M$. chiastus to the lowest occurrence of Quadrum gartneri.

Age. Late Cenomanian.

Remarks. Subzones $\mathrm{CC} 10 \mathrm{a}$ and $\mathrm{CC} 10 \mathrm{~b}$ are based on those of Manivit et al. (1977), which are assigned to Sissingh's (1977) number-coded scheme. Perch-Nielsen (1979) shows the first occurrence of Microrhab- dulus decoratus and $L$. acutus at the base of CC10. However, Verbeek (1977) and others show the first occurrence of $M$. decoratus in the upper middle Cenomanian to upper Cenomanian, above the first occurrence of L. acutus.

\section{LITHOSTRATIGRAPHY}

Because of drilling difficulties during Leg 103, a series of holes were drilled rather than the initially planned single reentry hole. As a result, we recovered sections that combine to form a complete, composite section with a complex history of sedimentation. As pointed out by Covington and Wise (1987), it has become increasingly important to pay close attention to lithologies and sedimentary environments in working with Lower Cretaceous nannofossils of the North Atlantic and Tethyan regions. Ranges of many species are not well known because of preservation, ecologic restriction, and/or a general lack of Lower Cretaceous sequences that contain diverse, well-preserved nannofossil assemblages. In addition, many of the sites drilled on the North Atlantic-Tethyan continental margins have recovered Neocomian, Wealden-type sequences characterized by deep-sea fan complexes consisting of nearshore sediments redeposited as turbidites. Reworking is common in these varied lithologies, and careful interpretations of the nannofossil biostratigraphy must be made. For this reason, the use of highest occurrences as datums, which are difficult to determine and apply, has been avoided as much as possible.

Figure 4 is a compilation of the composite sections from Sites 638 through 641 . Lithology, age, and depth are shown, along with general correlations among the local lithologic units and the oceanic formations proposed by Jansa et al. (1979) for the North Atlantic, where possible. A detailed description of the nannofossil assemblages is given in the next section of this paper (see "Biostratigraphy"), but a few general comments on the occurrence of key taxa in relation with lithology are included here.

Lithologic Unit III (Holes $641 \mathrm{~A}$ and $641 \mathrm{C}$ ): $149 \mathrm{~m}$ of middle Cretaceous (Aptian to lower Cenomanian) greenish gray and black claystone and calcareous clay and marl (53.9-202.6 m below seafloor (mbsf): Samples 103-641A-6X, CC (20 cm) to 103641C-6R-3, $58 \mathrm{~cm}$ [Hatteras Formation]).

The upper part of this unit, consisting of greenish calcareous clay and marl, contains a highly diverse and moderately wellpreserved nannofossil assemblage (latest Albian to early Cenomanian in age). The lower part of the unit, composed of dark, greenish gray and black claystones, contains assemblages in which diversity is greatly reduced and preservation is poorer. This indicates deposition either near or below the CCD, because the black claystones are generally barren whereas the lighter greenish claystones contain variable abundances of nannofossils.

Lithologic Unit IV (Hole 641C): $16 \mathrm{~m}$ of upper Aptian greenish gray marlstone and limestone conglomerate (calcareous turbidite) (202.6-218.3 mbsf: Samples 103-641C-6R-3, $58 \mathrm{~cm}$, to $103-641 \mathrm{C}-7 \mathrm{R}, \mathrm{CC}[11 \mathrm{~cm}])$.

The marlstone and limestone conglomerate contain well-preserved, diverse nannofossil assemblages that were redeposited as turbidites, as shown by the presence of early Barremian species in samples of late Aptian age from Section 103-641C-6R-3. One sample taken from inside a shell in a shell lag layer contains common Pickelhaube furtiva, a shallow-water form that is usually rare in other samples. It should be noted that the excellent preservation of the nannofossils in this redeposited material clearly demonstrates the minimal damage that nannofossils experience during transportation by turbidites. Therefore, nanno- 


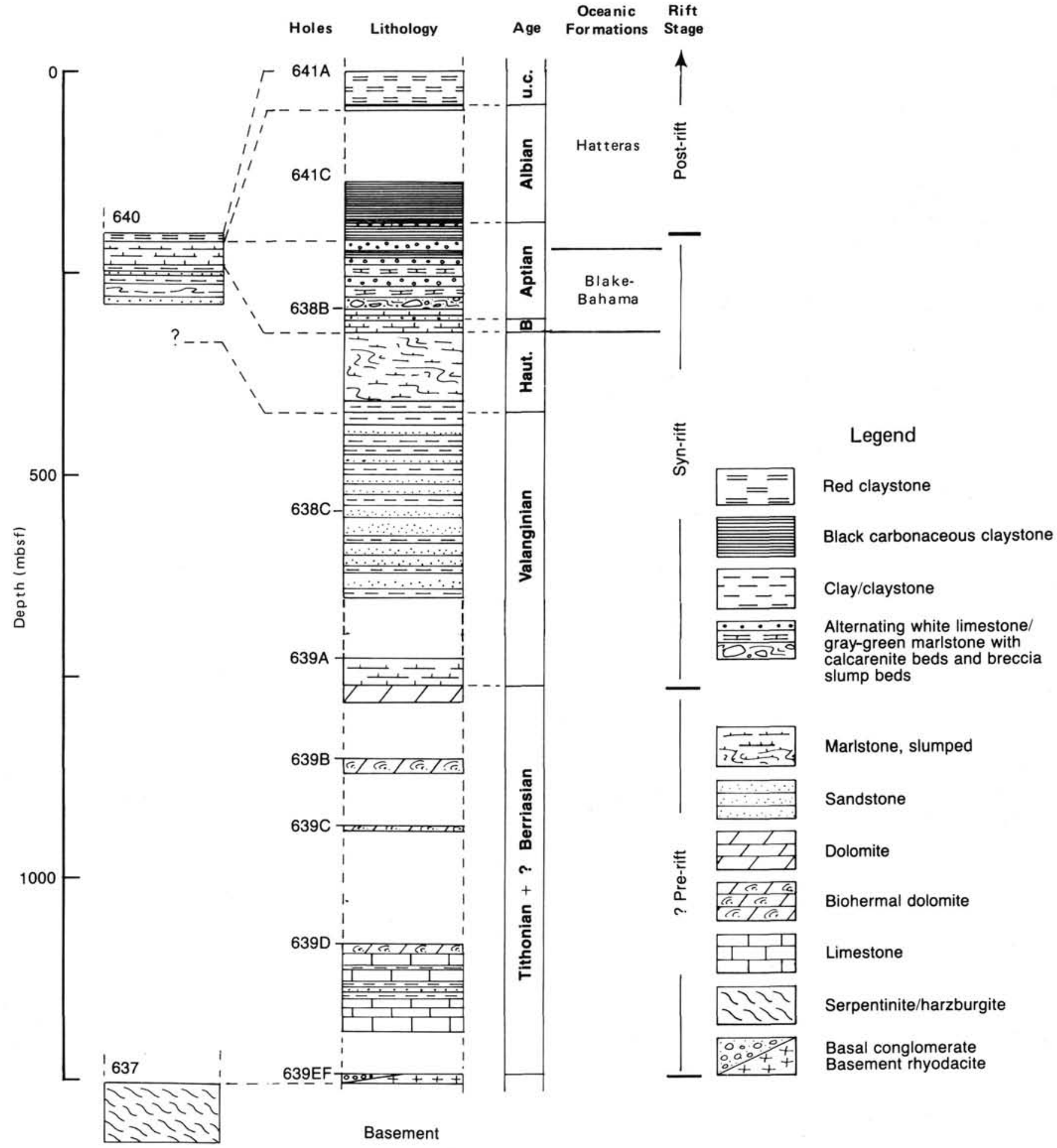

Figure 4. Compilation of composite sections including lithology, age, depth, and general correlations of lithologic units to oceanic formations.

fossil preservation generally should not be used as a factor in considering whether sediment has been redeposited.

Lithologic Unit V (Hole 641C): $32 \mathrm{~m}$ of lower Aptian, greenish gray calcareous microturbidites and marlstone (similar to part of the Hatteras Formation) (218.4-250.6 mbsf: Samples 103-641C-8R-1, $0 \mathrm{~cm}$, to $103-641 \mathrm{C}-11 \mathrm{R}-3,36 \mathrm{~cm}$ ).

Unit V consists of alternations of highly varied lithologies on a scale of 10 to $50 \mathrm{~cm}$. The upper part is less calcareous and contains more black carbonaceous claystone. Minor interbedded lithologies include calcarenite turbidites and debris flows containing limestone and marlstone clasts. At the top of this unit, the genera Micrantholithus, Nannoconus, and Lithraphidites dominate the nannofossil assemblages, in another demonstration of the introduction of neritic forms into the deep-sea environment. These shallow-water forms are found in high abundance in the lighter, more calcareous laminae of marl and limestone, where preservation is poor to moderate. The claystone 
and calcareous claystones contain better preserved nannofossils and fewer neritic forms, reflecting deposition in a more pelagic, open-ocean setting.

Lithologic Units VI (Hole 64IC) and IIA (Hole 638B): Up to $50 \mathrm{~m}$ of upper Barremian to lower Aptian alternating light gray bioturbated limestone and clayey limestone, laminated marlstone, and calcareous microturbidites (upper part similar to the Blake-Bahama Formation, overall intermediate between Hatteras and Blake-Bahama lithologies) (250.6-305.2 mbsf: Samples 103-641C-11R-3, $26 \mathrm{~cm}$, to 103-641C-16R, CC [22 cm]; 183.6212.6 mbsf: Section 103-638B-20R, CC, to Sample 103-638B$23 \mathrm{R}-3,3 \mathrm{~cm})$.

These units are interpreted as distal turbidite deposits interbedded with extremely bioturbated limestones and marlstones (see "Sedimentology" section of "Site 641C" chapter; Boillot, Winterer, et al., 1987). The nannofossil preservation within the bioturbated limestones is very poor owing to an extensive dissolution and reprecipitation process whereby nannofossil-bearing sediment is converted to limestone (Schlanger and Douglas, 1974; Wise, 1977). This process is even more accelerated within bioturbated sediment. The nannofossil assemblage is similar to the overlying unit.

Lithologic Subunit IIB (Hole 638B): $86 \mathrm{~m}$ of lower Hauterivian to lower Barremian alternations of light bioturbated nannofossil marlstone and darker gray, more calcareous-rich nannofossil marlstone, deformed in places by slumping (212.6-298.4 mbsf: Samples 103-638B-23R-3, $27 \mathrm{~cm}$, to 103-638B-32R-2, 95 $\mathrm{cm})$.

This subunit was deposited predominantly in a pelagic setting and contains a highly diverse, moderately well-preserved nannofossil assemblage. At the top of the unit the assemblage is dominated by the genera Nannoconus and Micrantholithus and L. bollii (thought to be shallow-water forms; Thierstein, 1976). The abundance of these genera increase upward from the bottom of Subunit IIB, which indicates either a decrease in water depth or an increase in the amount of shallow-water material shed into deep water.

\section{Lithologic Unit III (Site 638)}

Up to $140 \mathrm{~m}$ of lower Valanginian to lower Hauterivian alternations of thin-bedded calcareous claystone and nannofossil marlstone laminae interbedded with terrigenous sandstone (298.4547.2 mbsf: Samples 103-638B-32R-2, $9 \mathrm{~cm}$, to $103-638 \mathrm{C}-14 \mathrm{R}$, $\mathrm{CC}[30 \mathrm{~cm}])$.

The lower Subunit IIIB is distinguished from the overlying Subunit IIIA by numerous, thick beds of terrigenous sandstone. The top of the highest thick sandstone bed is at $55 \mathrm{~cm}$ in Section 103-638B-35R-4. The nannofossil marlstone directly above Subunit IIIB contains the most diverse and well-preserved nannofossil assemblage in the Neocomian section. The diversity and overall species composition of the nannofossil assemblages change drastically in the lower sandstone turbidite subunit, where the nannoconids and micrantholiths are greatly reduced in numbers. The sandstone and claystone in Subunit IIIB are interpreted as turbidites, whereas the laminae of nannofossil marlstone are interpreted as representing background pelagic sedimentation (see "Sedimentology" section of "Site 638" chapter; Boillot, Winterer, et al., 1987). The marked decrease of nannofossils in the sandstone turbidites can be attributed to a high sedimentation rate for terrigenous material and a relatively small amount of pelagic deposition.

\section{Lithologic Unit III (Hole 639A)}

$49 \mathrm{~m}$ of lower Valanginian pale yellow nannofossil marl/ marlstone (21.6-70.3 mbsf: Samples 103-639A-4R-1, $10 \mathrm{~cm}$, to 103-639A-8R, CC [7 cm]).

Nannoconids dominate assemblages in the nannofossil marl/ marlstone. Change in the degree of lithification, and thus preservation, is observed downhole. A transition from soft marl to dominantly hard marlstone in Core 103-639A-7R is accompanied by a change in nannofossil preservation from poor to very poor. The triad of early Valanginian markers $C$. oblongata, $D$. rectus, and $T$. verenae is absent below this horizon, probably as a result of the changes in lithification and preservation.

A direct relationship between nannofossil content and lithology is observed in the varied and complex Lower Cretaceous sequence. Besides preservational changes, which are related to the higher calcite content and lithification, increased numbers of shallow-water forms redeposited from the upper slope-outer shelf to deeper water by way of turbidity flows, creeps, and slumps are observed. Despite the large amount of redeposited material, a nearly continuous stratigraphic section can be pieced together. The only significant hiatus detected in this sequence is where the upper Hauterivian-lower Barremian is missing at Holes 638B and 640A. These holes are approximately $31 \mathrm{~km}$ apart, demonstrating that this event may have occurred on a regional scale.

A close analogy to this sequence is that of Hole 637A (Wei et al., this volume), which contains redeposited material but is stratigraphically continuous. In Hole 637A, although upper Miocene to Recent siltstones, claystones, and nannofossil marls and oozes were redeposited into very deep water $(\sim 5000 \mathrm{~m})$ by turbidity flows, a stratigraphically intact and nearly complete sequence is present with only one well-documented hiatus. Much of the material in these sections was redeposited, but the redeposition appears consistent and continuous, making use of the nannofossil biostratigraphy possible.

\section{BIOSTRATIGRAPHY}

As stated previously, a composite Lower Cretaceous sequence can be constructed from Holes 639A, 638B, 638C, 640A, 641A, and $641 \mathrm{C}$. The range charts for each of these holes are in Tables 1-5 and include the total and individual species abundances, as well as the zonal assignments and ages for all samples examined. In the following biostratigraphic discussion, the five holes that make up this composite section are treated as one continuous section. Figure 5 shows the ranges of index nannofossils and other important species.

The oldest Early Cretaceous nannofossils recovered on ODP Leg 103 were from the calpionellid, nannofossil marlstone of Hole 639A. The occurrence of $C$. oblongata, $T$. verenae, and $D$. rectus in Sample 103-639A-7R-3, 101-102 cm, indicates an age no older than early Valanginian. Cyclagelosphaera brezae n. sp. and Eiffellithus primus n. sp. are present in many of the marlstone samples. Below Core 103-639A-7R, preservation worsens and assemblages are dominated by nannoconids and Watznaueria barnesae. The early Valanginian age determined from the nannofossils in this section is in good agreement with that given by the calpionellid and foraminiferal data (see "Biostratigraphy" section of "Site 639" chapter; Boillot, Winterer, et al., 1987).

Above the calpionellid-nannofossil marlstone of Hole 639A lies the sand turbidite sequence recovered in Holes 638B and $638 \mathrm{C}$. The contact between the strikingly different lithologies was not recovered (the hole caved in and was abandoned just above this contact in Hole $638 \mathrm{C}$ ). The diversity of nannofossils decreases and preservation worsens from the bottom of Hole 
Table 1. Relative abundance of nannofossils, Hole 639A.

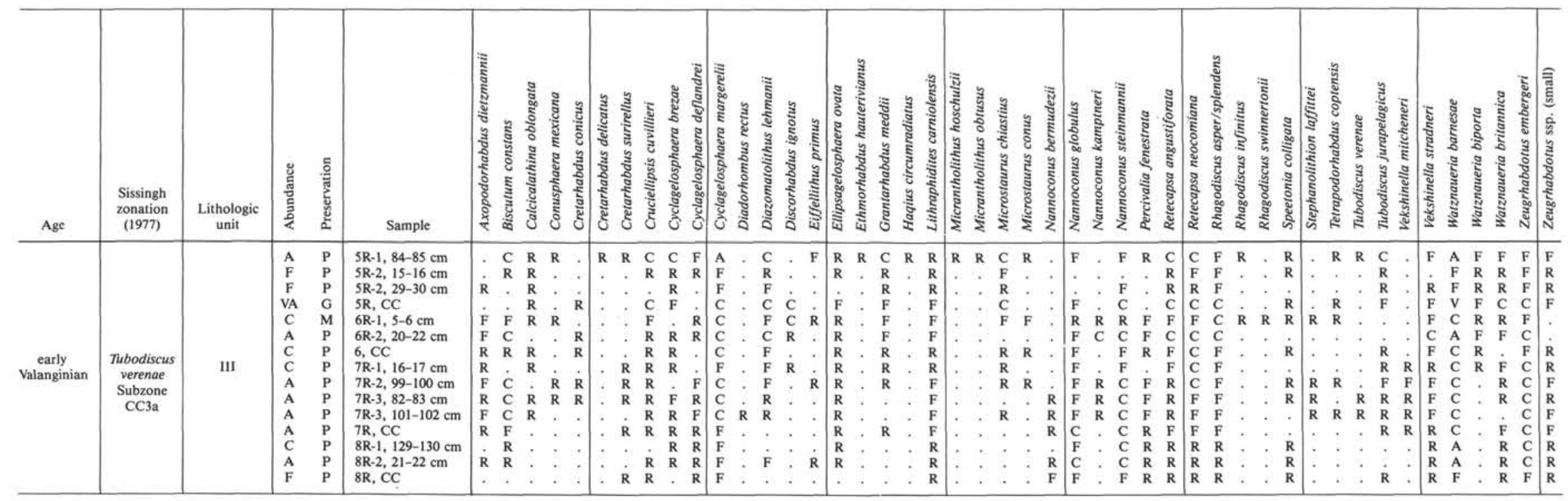

$\mathrm{R}=$ Rare; $\mathrm{F}=$ Few; $\mathrm{C}=$ Common; $\mathrm{A}=$ Abundant; $\mathrm{VA}=$ Very abundant; ? = Questionably present;.$=$ Not present. 
$638 \mathrm{C}$ to the top of Hole 639A. The vertical distance between the two holes, calculated aboard ship from seismic sections, could be as little as $10 \mathrm{~m}$. No missing section is evident from the distribution of the marker nannofossil species, although many other species first appear at the bottom of Hole $638 \mathrm{C}$, including: $\mathrm{No}$ dosella silvaradion, Discorhabdus biradiatus, Glaukolithus diplogrammus, Retecapsa radiata, Octopodorhabdus plethotretus, P. furtiva, Vekshinella parallela, and Zeugrhabdotus pseudoangustus. The first appearance of these taxa could be due to a small hiatus or a change in preservation.

Thierstein (1976) illustrated the first occurrences of $R$. radiata and $G$. diplogrammus near the middle of the Valanginian. Rucinolithus wisei, a species that has its last occurrence in the middle Valanginian, is present at the bottom of Hole 638B but is not a useful datum here because of its rare occurrence within the turbidite sequence. Roth (1983) placed the first occurrence of Rhagodiscus infinitus above the last occurrence of $R$. wisei; however, in this section the two species clearly overlap in occurrence as they also do at DSDP Site 603 (Covington and Wise, 1987). Bralower (1986) observed $R$. infinitus below the Valanginian, which makes its first occurrence a doubtful upper Valanginian marker.

A mid-Valanginian marker proposed here is the first occurrence of $E$. windii n. sp. This small distinctive Eiffellithus was first illustrated by Wind and Cepek (1979) for the Hauterivian at Site 397 and later by Covington and Wise (1987) from the Hauterivian at Site 603. Although it is rare to few in abundance in our material, $E$. windii n. sp. has a consistent occurrence and is a highly distinct form that birefringes strongly (bright white) in polarized light.

The first occurrence of Tegumentum stradneri in Zone CC3b extends the lower range of this species into the upper Valanginian. The last occurrence of C. brezae n. sp. in Sample 103638B-33R-1, 75-76 cm, corresponds with the lowest occurrence of E. striatus, the datum used here to mark the Valanginian/ Hauterivian boundary. $C$. brezae has its highest occurrence three cores above that of $C$. deflandrei.

In previous studies of Lower Cretaceous nannofossils many different datums were used to delineate the Valanginian/Hauterivian boundary. The last occurrences of $T$. verenae and $D$. rectus in Roth's (1978) zonal scheme cannot be used because of their occurrence in the upper Hauterivian and Barremian elsewhere. It is interesting to note that the highest common occurrence of $T$. verenae in this section (Sample 103-638B-32R-2, 8-9 $\mathrm{cm})$ is roughly coincident with the lowest occurrence of $E$. striatus. Last-appearance datums must be used with caution in areas where the possibility of reworking is great. It is for this reason that the lowest occurrence of $E$. striatus is used to approximate the Valanginian/Hauterivian boundary.

The first occurrence of C. loriei was used by Sissingh (1977) for the Valanginian/Hauterivian boundary. A form that resembles $C$. loriei was observed in Hauterivian material from this study (Cretarhabdus cf. loriel); however, the central area and overall size of this form are not as large as those of the holotype, and the parallel laths in each of the four quadrants are not nearly as well defined as in $C$. loriei. Forms closely resembling $C$. loriei have been observed by one of the authors from the Upper Jurassic of Portugal. Unfortunately, Sissingh (1977) did not illustrate the form he identified as $C$. loriei. Thus, until further taxonomic clarification is made, this is considered an unreliable datum for the $\mathrm{CC} 3 / \mathrm{CC} 4$ boundary.

The first occurrence of $L$. bollii marks the base of $C C 4 \mathrm{~b}$ in Sample 103-638B-29-4, 18-19 cm. Our specimens are clearly synonymous with the species described by Thierstein (1971) and not the form discussed as L. bollii? by Wind and Cepek (1979). The upper limit of $\mathrm{CC} 4 \mathrm{~b}$, based on the last occurrence of $S$. col- ligata, extends up to Sample 103-638B-23R-4, 55-56 cm. An unconformity is recognized between lithologic Subunits IIA and IIB at 278 mbsf in Section 103-638B-23R-3, where part of the upper Hauterivian and most of the lower Barremian are not detected. $L$. bollii and $C$. oblongata both appear to have their highest occurrences truncated in this section and occur in Sample 103-638B-23R-3, 16-17 cm, one core section above the highest occurrence of $S$. colligata. Species that have their lowest occurrence in CC4b include Flabellites oblongus and Nannoconus liqius $\mathrm{n}$. sp. Cruciellipsis cuvillieri has its highest occurrence approximately $10 \mathrm{~m}$ below the highest occurrence of $S$. colligata in Sample 103-638B-25R-1, 86-87 cm.

Nannofossil assemblages in $\mathrm{CC} 4 \mathrm{~b}$ are dominated by the genera Nannoconus, Micrantholithus, Lithraphidites, and Watznaueria. Preservation is good, except in the bioturbated intervals, and many samples contain a background carbonate hash composed of broken micrantholith elements. Although generally good, preservation is often variable within each sample, a phenomenon also noted by Wind and Cepek (1979). Above the unconformity between lithologic Subunits IIA and IIB, upper Barremian nannofossil assemblages are present to the top of the Lower Cretaceous sequence of Hole 638B. The lower part of Hole 641C (Sample 103-641C-11R-2, $108 \mathrm{~cm}$, to Section 103$641 \mathrm{C}-16 \mathrm{R}, \mathrm{CC}$ ) contains very similar assemblages in a lithology resembling that of the upper Barremian in Hole 638B. In the absence of both $C$. oblongata and $H$. irregularis, these intervals are assigned to the $M$. hoschulzii Zone (CC6). Species present in this interval include Tubodiscus jurapelagicus, C. litterarius, rare $D$. rectus, $N$. abundans, and $R$. radiata. Rucinolithus terebrodentarius, a form that could be confused under the light microscope with $H$. irregularis, has its lowest occurrence within this zone.

Recognition of the Barremian/Aptian boundary has long been a problem for nannofossil biostratigraphers. Thierstein (1971) first used the first occurrence of $C$. litterarius, a form described from the Maestrichtian by Gorka (1957). Forms observed in the Hauterivian and Barremian of the Leg 103 material easily fit within Thierstein's (1971) species concept of C. litterarius. The last occurrence of Nannoconus steinmannii/colomii, also proposed by Thierstein (1971) to mark the boundary, ranges up to the middle Aptian in this section.

The first occurrence of $H$. irregularis is used in this study to define the Barremian/Aptian boundary. Roth (1983) extended the range of this species down into the upper Barremian. However, under the light microscope, it can be confused with $R$. terebrodentarius, which can resemble an overgrown $H$. irregularis (see taxonomic notes). Therefore, we do not agree that $H$. irregularis occurs below the $\mathrm{CC} 6 / \mathrm{CC} 7$ boundary. The $H$. irregularis Subzone (CC7a), is assigned to the interval from Samples 103$641 \mathrm{C}-11 \mathrm{R}-2,108 \mathrm{~cm}$, to $103-641 \mathrm{C}-8 \mathrm{R}-4,76-77 \mathrm{~cm}$. Flabellites biforaminis, a form previously restricted to the Aptian, has its first occurrence one sample below this interval in Sample 103$641 \mathrm{C}-12 \mathrm{R}-3,107 \mathrm{~cm}$. Many taxa disappear near the top of CC7a, including $M$. hoschulzii, M. obtusus, N. steinmannii, Nannoconus globulus, and $C$. mexicana. Some of these are found reworked with Barremian forms in a few samples zoned within CC7b.

The interval from Samples 103-641C-8R-4, 76-77 $\mathrm{cm}$, to 103-641C-6R-3, $77 \mathrm{~cm}$, is assigned to the $R$. angustus Subzone (CC7b). Layers of coarse shell debris in a carbonate turbidite sequence are found at the top of this interval. The common occurrence of $P$. furtiva within a shell lag layer indicates that this species is a nearshore form. The presence of definitive lower Barremian nannofossils in this lag layer indicate that $P$. furtiva is also reworked, as it is otherwise restricted to Barremian age or older sediment. 
Table 2. Relative abundance of nannofossils, Site 638.

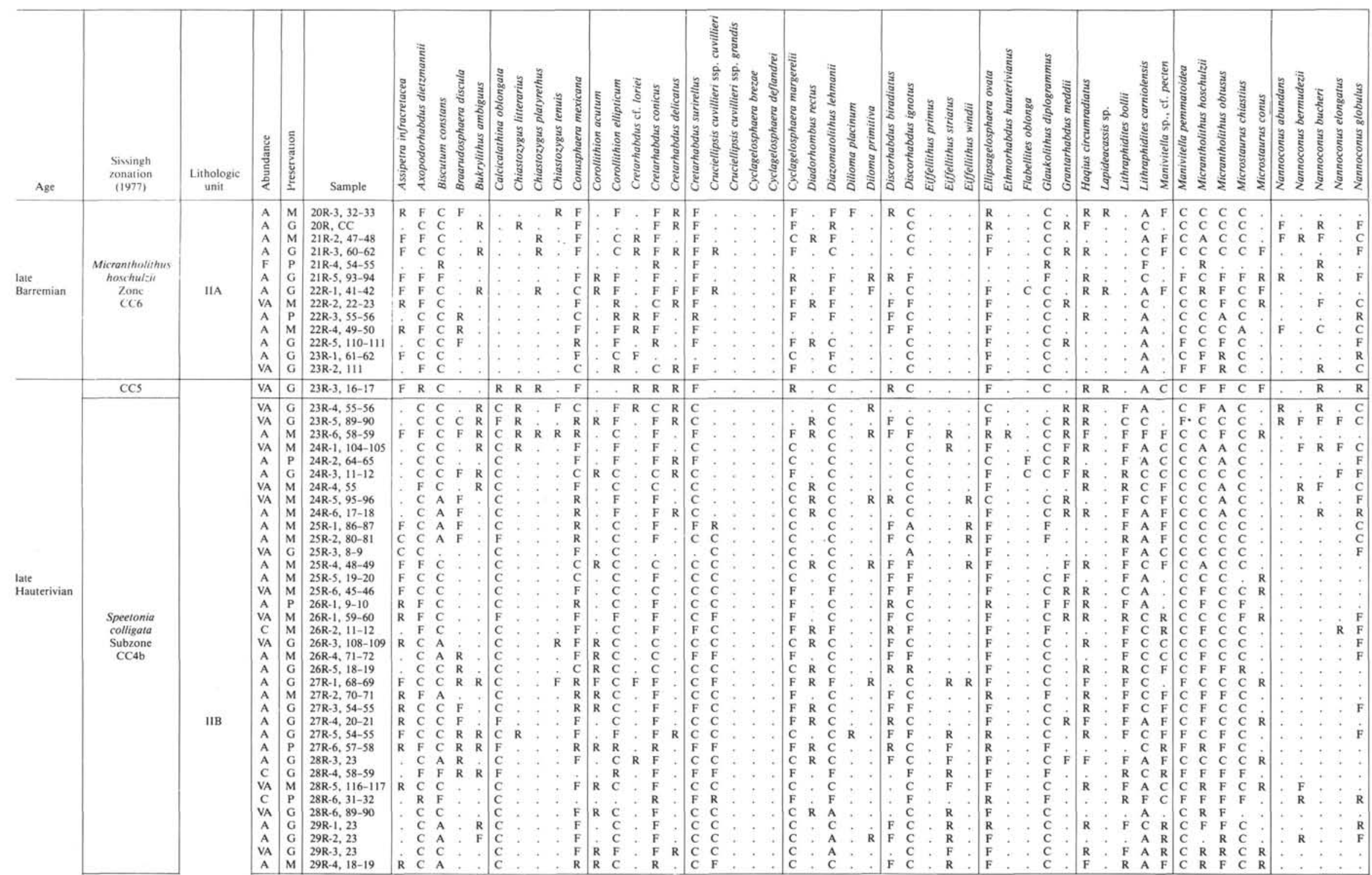


Table 2 (continued).

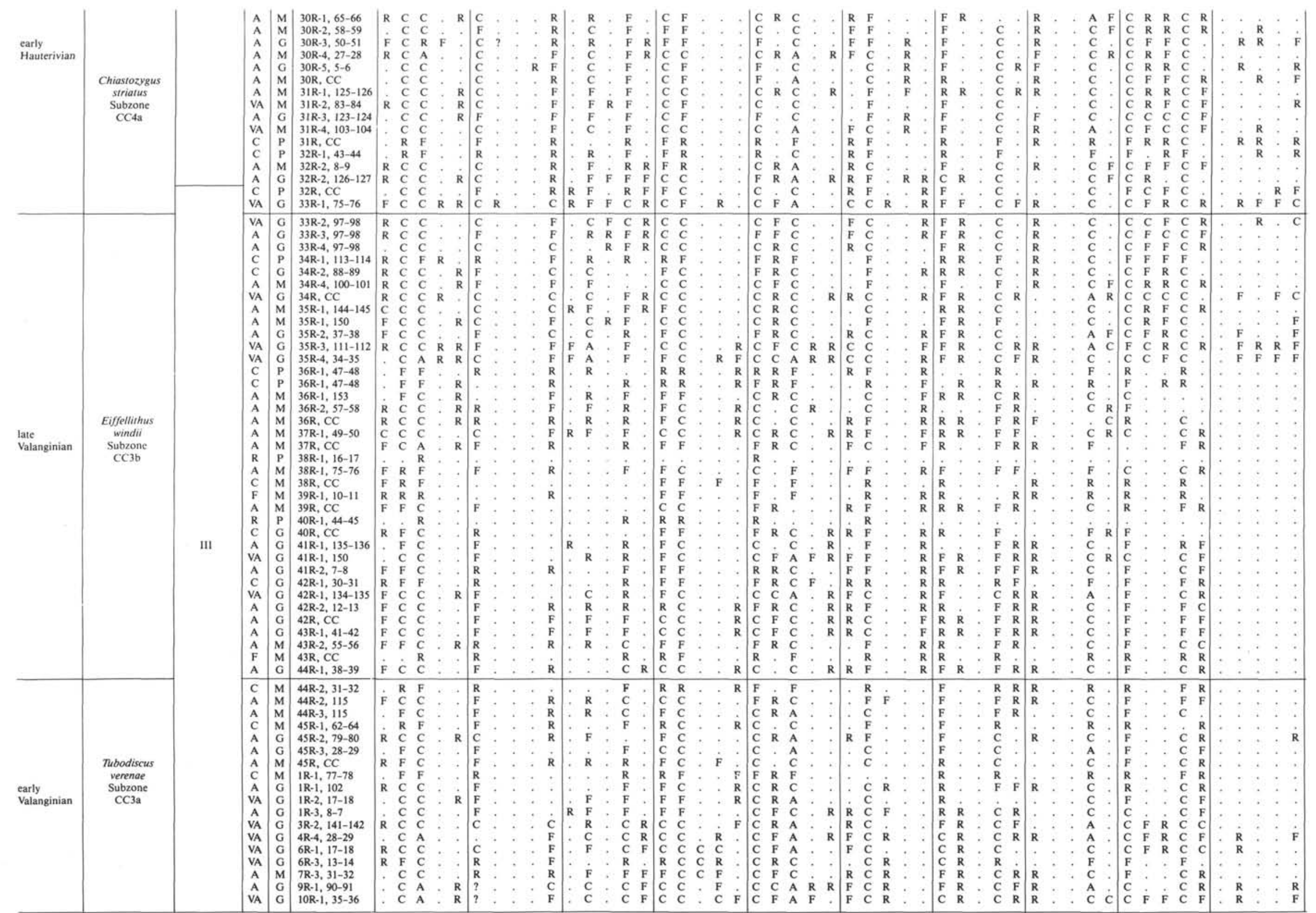


Table 2 (continued).

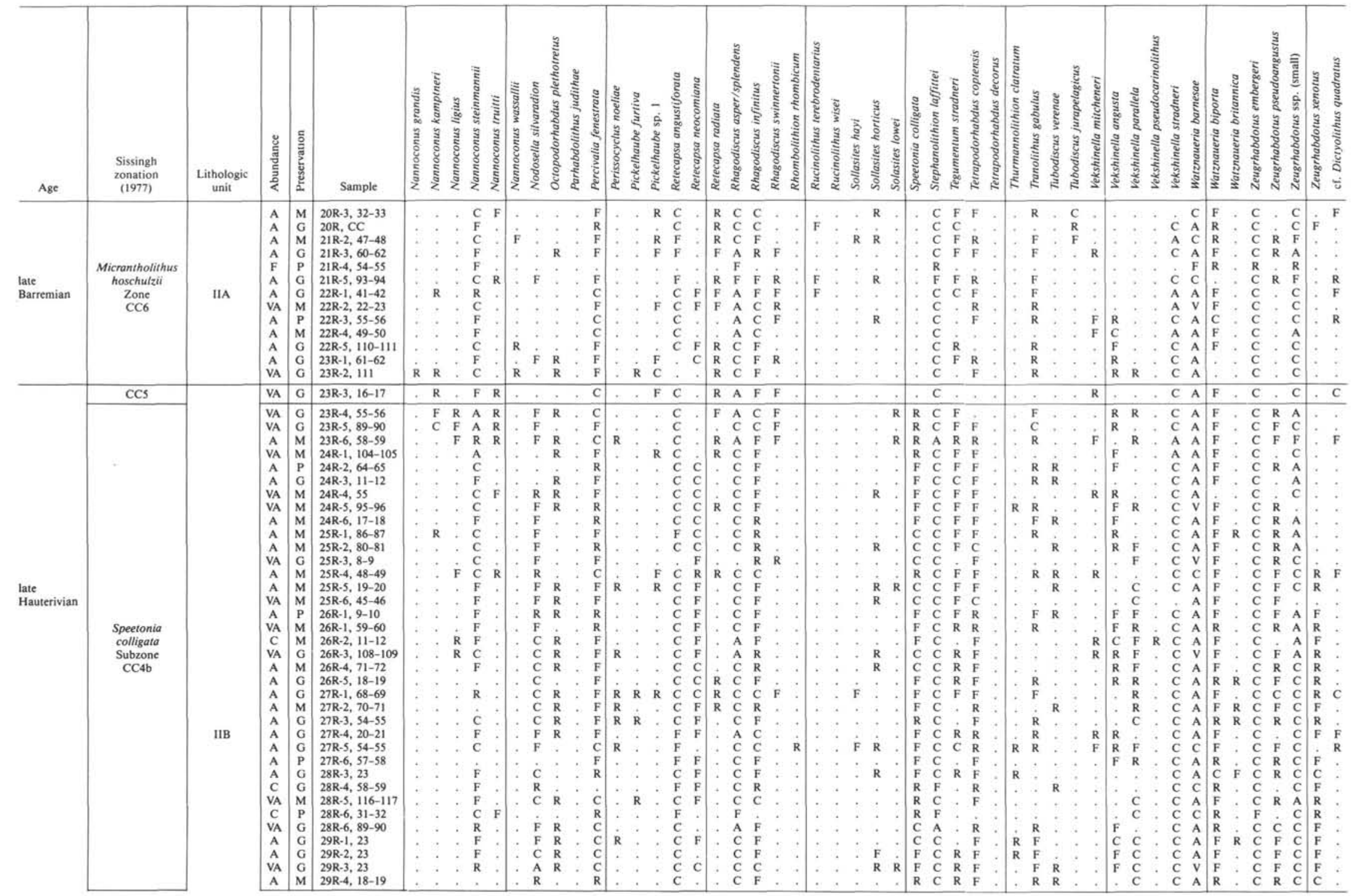


Table 2 (continued).

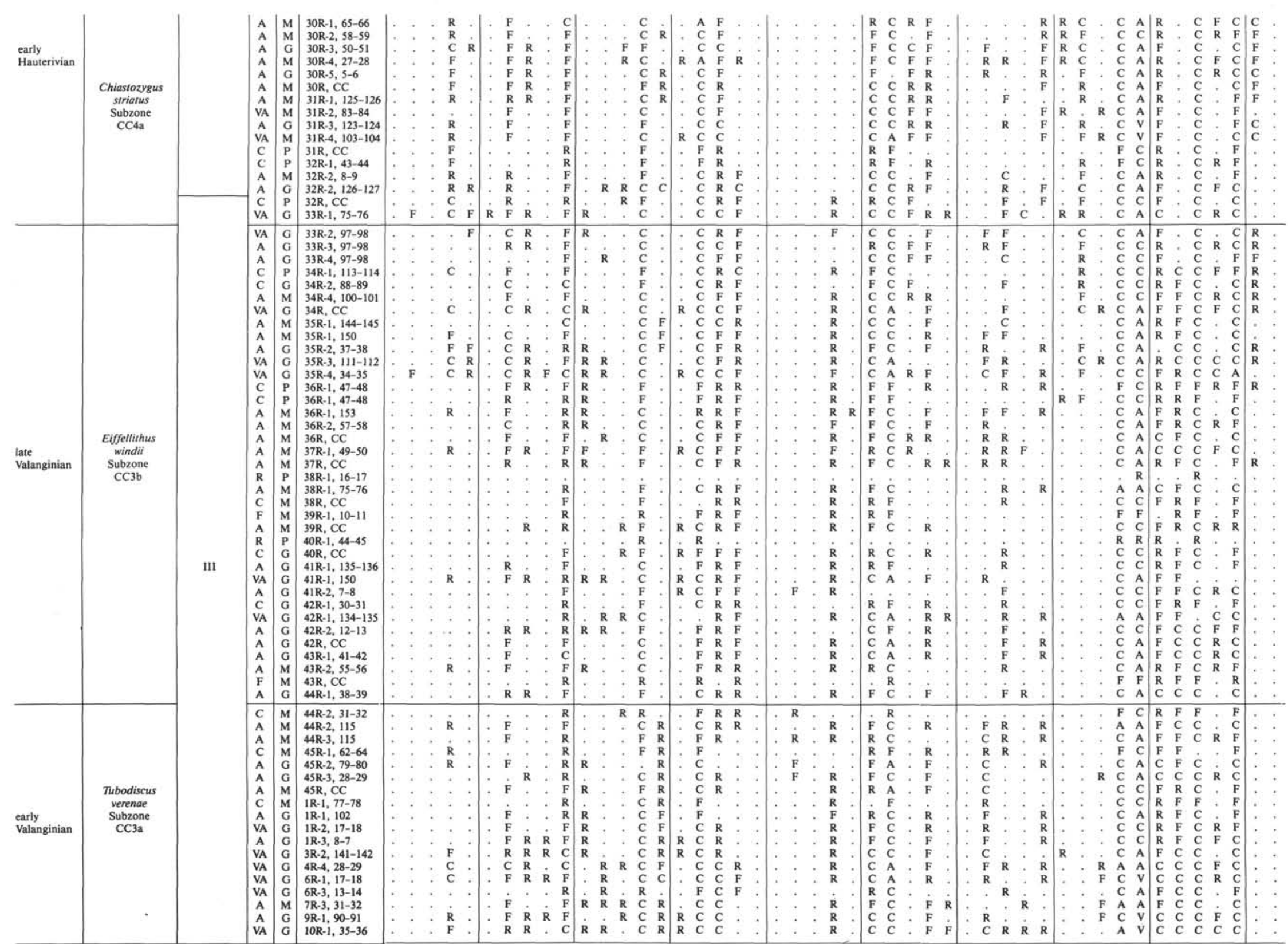


Table 3. Relative abundance of nannofossils, Site 640A.

\begin{tabular}{|c|c|c|c|c|c|c|c|c|c|c|c|c|c|c|c|c|c|c|c|c|c|c|c|c|c|c|c|c|c|c|c|c|c|c|c|c|}
\hline Age & $\begin{array}{c}\text { Sissingh } \\
\text { zonation } \\
\text { (1977) }\end{array}$ & $\begin{array}{l}\text { Lithologic } \\
\text { unit }\end{array}$ & 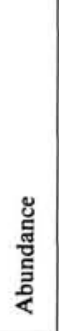 & 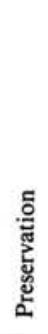 & Sample &  & & $\frac{5}{3}$ & & 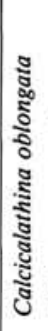 & 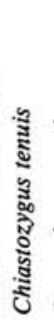 & &  & 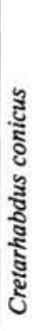 & 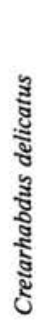 & $\begin{array}{l}\text { है } \\
\text { हैँ } \\
\text { हूँ } \\
\text { हूँ }\end{array}$ & 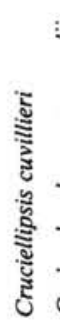 & 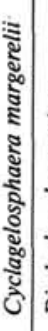 & 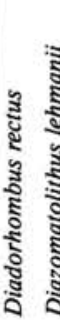 & 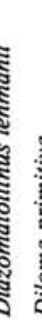 & 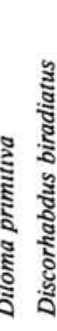 & 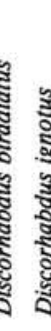 & & 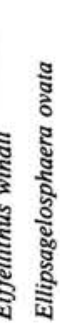 & 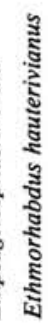 & 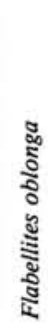 & 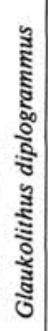 & 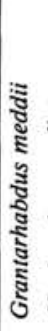 & $\begin{array}{l}5 \\
3 \\
3\end{array}$ & 厸: & 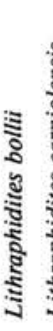 & 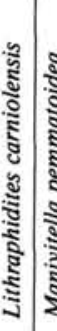 & 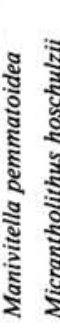 & 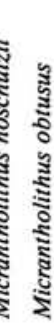 & 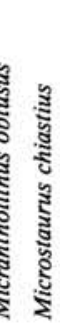 & 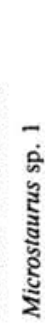 \\
\hline $\begin{array}{c}\text { late } \\
\text { Barremian }\end{array}$ & $\begin{array}{c}\text { Micrantho- } \\
\text { lithus } \\
\text { hoschulzii } \\
\text { Zone } \\
\text { CC6 }\end{array}$ & \multirow[b]{3}{*}{ II } & $\begin{array}{l}\text { A } \\
\text { A } \\
\text { A } \\
\text { A } \\
\text { A } \\
\text { A }\end{array}$ & $\begin{array}{l}\mathrm{P} \\
\mathrm{M} \\
\mathrm{M} \\
\mathrm{G} \\
\mathrm{G} \\
\mathrm{G}\end{array}$ & $\begin{array}{l}2 \mathrm{R}-2,60 \mathrm{~cm} \\
2 \mathrm{R}, \mathrm{CC} \\
2 \mathrm{R}, \mathrm{CC}(29 \mathrm{~cm}) \\
3 \mathrm{R}-2,68 \mathrm{~cm} \\
3 \mathrm{R}-3,56-57 \mathrm{~cm} \\
3 \mathrm{R}, \mathrm{CC}\end{array}$ & $\begin{array}{ll}\mathrm{R} & \mathrm{R} \\
\dot{\mathrm{F}} & \mathrm{R} \\
\text {. } & \mathrm{F} \\
: & \mathrm{C} \\
. & \mathrm{C} \\
\end{array}$ & $\begin{array}{l}\mathrm{F} \\
\mathrm{C} \\
\mathrm{C} \\
\mathrm{C} \\
\mathrm{C}\end{array}$ & : & $\begin{array}{l}\mathrm{R} \\
\dot{\mathrm{R}} \\
\mathrm{R} \\
.\end{array}$ & : & $\begin{array}{l}\mathrm{R} \\
\mathrm{R} \\
\dot{\mathrm{R}} \\
\dot{.}\end{array}$ & $\begin{array}{l}\mathrm{C} \\
\mathrm{F} \\
\mathrm{F}\end{array}$ & $\begin{array}{ll}: & : \\
: & \mathrm{F} \\
\dot{\mathrm{F}} & \mathrm{R} \\
\end{array}$ & $\begin{array}{l}\text { F } \\
\text { F } \\
\text { C } \\
\text { F } \\
\text { F } \\
\text { C }\end{array}$ & : & $\dot{:}$ & $: 5$ & & $\begin{array}{l}\mathrm{F} \\
: \quad \mathrm{C} \\
: \quad \mathrm{F} \\
: \quad \mathrm{F} \\
\end{array}$ & &  & $\begin{array}{ll} & F \\
. & C \\
. & C \\
F & C \\
F & C\end{array}$ & & $:$ & : & $\begin{array}{l}\dot{\mathrm{R}} \\
\vdots\end{array}$ & $\begin{array}{l}\dot{R} \\
\mathrm{R} \\
\mathrm{C} \\
\mathrm{C} \\
\mathrm{C}\end{array}$ & $\begin{array}{l}\mathrm{R} \\
\mathrm{R} \\
\mathrm{R} \\
\cdot \\
\end{array}$ & $\begin{array}{l}\dot{2} \\
\dot{\mathrm{R}} \\
\mathrm{R} \\
\mathrm{R} \\
\text {. }\end{array}$ & i. & $: 5$ & \begin{tabular}{l|l}
$\mathrm{C}$ & \\
$\mathrm{C}$ & \\
$\mathrm{C}$ & \\
$\mathrm{C}$ & \\
$\mathrm{C}$ & \\
$\mathrm{C}$ & $\mathbf{c}$ \\
\end{tabular} & $\begin{array}{ll}\mathrm{C} & \mathrm{F} \\
\mathrm{C} & \mathrm{C} \\
\mathrm{C} & \mathrm{F} \\
\mathrm{C} & \mathrm{C} \\
\mathrm{C} & \mathrm{C} \\
\mathrm{C} & \mathrm{C} \\
\end{array}$ & $\begin{array}{ll}\mathrm{F} & \mathrm{F} \\
\mathrm{R} & \mathrm{R} \\
\mathrm{F} & \mathrm{C} \\
\mathrm{C} & \mathrm{C} \\
\mathrm{C} & \mathrm{C} \\
\mathrm{C} & \mathrm{C} \\
\end{array}$ & $\begin{array}{ll}\mathrm{F} & \mathrm{R} \\
\mathrm{R} & \mathrm{F} \\
\mathrm{C} & \mathrm{C} \\
\mathrm{C} & \mathrm{C} \\
\mathrm{C} & \mathrm{C} \\
\mathrm{C} & \mathrm{C} \\
\end{array}$ & : \\
\hline \multirow[b]{2}{*}{$\begin{array}{c}\text { late } \\
\text { Hauterivian }\end{array}$} & $\begin{array}{c}\text { Lithraphidites } \\
\text { bollii } \\
\text { Zone } \\
\text { CC5 }\end{array}$ & & $\begin{array}{l}\text { VA } \\
\text { A } \\
\text { VA } \\
\text { VA } \\
\text { A } \\
\end{array}$ & $\begin{array}{l}\text { G } \\
\text { M } \\
\text { G } \\
\text { G } \\
\text { G }\end{array}$ & $\begin{array}{l}4 \mathrm{R}-1,104-105 \mathrm{~cm} \\
4 \mathrm{R}-2,9-10 \mathrm{~cm} \\
4 \mathrm{R}, \mathrm{CC} \\
5 \mathrm{R}-1,3 \mathrm{~cm} \\
5 \mathrm{R}-1,7 \mathrm{~cm}\end{array}$ & $\begin{array}{ll}: & \mathrm{C} \\
\mathrm{R} & \mathrm{F} \\
: & \mathrm{F} \\
: & \mathrm{C} \\
: & \mathrm{F} \\
\end{array}$ & $\begin{array}{l}\mathrm{C} \\
\mathrm{C} \\
\mathrm{C} \\
\mathrm{C} \\
\mathrm{C}\end{array}$ & $\begin{array}{l}\mathrm{R} \\
\dot{\mathrm{R}} \\
\dot{\mathrm{R}}\end{array}$ & $\begin{array}{r}\mathrm{R} \\
\dot{\mathrm{R}} \\
\dot{\mathrm{R}} \\
\mathrm{R} \\
\end{array}$ & $\begin{array}{l}\mathrm{F} \\
\mathrm{R} \\
\mathrm{F} \\
\mathrm{R} \\
\mathrm{C} \\
\end{array}$ & $\begin{array}{l}\dot{2} \\
\dot{R} \\
\mathrm{R} \\
\mathrm{R}\end{array}$ & $\begin{array}{l}\mathrm{R} \\
\mathrm{R} \\
\mathrm{F} \\
\mathrm{R} \\
\mathrm{F}\end{array}$ & $\begin{array}{ll}\dot{1} & \dot{\mathrm{C}} \\
\dot{5} & \mathrm{~F} \\
\dot{\mathrm{R}} & \mathrm{F} \\
\end{array}$ & $\begin{array}{l}\mathrm{C} \\
\mathrm{C} \\
\mathrm{F} \\
\mathrm{C} \\
\mathrm{F}\end{array}$ & $\begin{array}{l}\dot{2} \\
\dot{\mathrm{R}} \\
\mathrm{R} \\
\mathrm{F}\end{array}$ & $\begin{array}{l}\mathrm{C} \\
\mathrm{F} \\
\mathrm{F} \\
\mathrm{R} \\
\mathrm{C}\end{array}$ & : & \begin{tabular}{l|l}
$C$ & \\
$C$ & \\
$C$ & \\
$C$ & \\
$C$ & \\
\end{tabular} & $\begin{array}{ll}\dot{R} & \mathrm{C} \\
\mathrm{R} & \mathrm{C} \\
\mathrm{R} & \mathrm{C} \\
\dot{\mathrm{F}} & \mathrm{C} \\
\dot{\mathrm{F}} & \mathrm{A} \\
\end{array}$ & $\begin{array}{lll}C & & \\
c & 1 \\
C & 1 & \\
C & \\
\text { A } & & \\
& & \end{array}$ & $\begin{array}{ll}\dot{R_{R}} & \mathrm{~F} \\
\mathrm{R} & \mathrm{F} \\
\mathrm{R} & \mathrm{F} \\
\dot{\mathrm{R}} & \mathrm{F} \\
\mathrm{R} & \mathrm{C} \\
\end{array}$ & $\begin{array}{ll}F & C \\
F & c \\
F & C \\
F & C \\
C & C \\
\end{array}$ & $\begin{array}{l}R \\
R\end{array}$ & $\begin{array}{l:}\cdot \\
\dot{R} \\
\mathrm{R}\end{array}:$ & $\dot{\mathrm{R}}$ & : & $\begin{array}{l}\mathrm{C} \\
\mathrm{C} \\
\mathrm{C} \\
\mathrm{C} \\
\mathrm{C} \\
\end{array}$ & $\begin{array}{l}\mathrm{R} \\
\mathrm{R} \\
\mathrm{F} \\
\mathrm{R} \\
\mathrm{F} \\
\end{array}$ & $\begin{array}{l}\dot{R} \\
\mathrm{R} \\
\mathrm{F} \\
\mathrm{R}\end{array}$ & $\dot{\mathrm{R}}$ & : & \begin{tabular}{l|l}
$c$ & \\
C & \\
C & \\
c & \\
c & \multicolumn{1}{c}{} \\
\end{tabular} & $\begin{array}{ll}\mathrm{C} & \mathrm{A} \\
\mathrm{C} & \mathrm{F} \\
\mathrm{C} & \mathrm{F} \\
\mathrm{C} & \mathrm{R} \\
\mathrm{C} & \mathrm{F} \\
\end{array}$ & $\begin{array}{ll}\mathrm{A} & \mathrm{C} \\
\mathrm{F} & \mathrm{R} \\
\mathrm{F} & \mathrm{F} \\
\mathrm{R} & \mathrm{F} \\
\mathrm{F} & \mathrm{C}\end{array}$ & $\begin{array}{ll}C & C \\
R & C \\
F & C \\
. & C \\
C & F \\
\end{array}$ & \\
\hline & $\begin{array}{c}\text { Speetonia } \\
\text { colligata } \\
\text { Subzone } \\
\text { CC4b }\end{array}$ & & $\begin{array}{l}\text { A } \\
\text { A } \\
\text { A } \\
\text { A } \\
\text { A } \\
\text { A } \\
\text { A } \\
\text { A } \\
\text { A } \\
\text { A } \\
\text { VA } \\
\text { C }\end{array}$ & $\begin{array}{l}\text { G } \\
\text { G } \\
\text { G } \\
\text { G } \\
\text { M } \\
\text { M } \\
\text { P } \\
\text { G } \\
\text { M } \\
\text { M } \\
\text { G } \\
\text { P }\end{array}$ & $\begin{array}{l}5 \mathrm{R}-1,22 \mathrm{~cm} \\
5 \mathrm{R}, \mathrm{CC} \\
6 \mathrm{R}-1,50 \mathrm{~cm} \\
6 \mathrm{R}-1,120 \mathrm{~cm} \\
6 \mathrm{R}, \mathrm{CC} \\
7 \mathrm{R}-1,115-116 \mathrm{~cm} \\
7 \mathrm{R}-2,10-11 \mathrm{~cm} \\
7 \mathrm{R}, \mathrm{CC} \\
7 \mathrm{R}, \mathrm{CC} \\
8 \mathrm{R}-1,8-9 \mathrm{~cm} \\
9 \mathrm{R}-1,1 \mathrm{~cm} \\
8 \mathrm{R}, \mathrm{CC}(5-6 \mathrm{~cm})\end{array}$ & $\begin{array}{ll}: & \mathrm{C} \\
: & \mathrm{C} \\
: & \mathrm{C} \\
: & \mathrm{F} \\
: & \mathrm{F} \\
: & \mathrm{F} \\
: & \mathrm{F} \\
: & \mathrm{F} \\
: & \mathrm{C} \\
\mathrm{R} & \mathrm{R} \\
\mathrm{R} & \mathrm{C}\end{array}$ & $\begin{array}{l}\text { C } \\
\text { C } \\
\text { A } \\
\text { C } \\
\text { C } \\
\text { C } \\
\text { C } \\
\text { C } \\
\text { C } \\
\text { C } \\
\text { F } \\
\text { C }\end{array}$ & $\begin{array}{l}\mathrm{R} \\
. \\
\text {. } \\
\text {. }\end{array}$ & $\begin{array}{l}\mathrm{R} \\
\mathrm{R} \\
\mathrm{R} \\
\mathrm{R} \\
\mathrm{F} \\
\mathrm{R}\end{array}$ & $\begin{array}{l}\mathrm{F} \\
\mathrm{R} \\
\mathrm{F} \\
\mathrm{C} \\
\mathrm{F} \\
\mathrm{F} \\
\mathrm{C} \\
\mathrm{C} \\
\mathrm{C} \\
\mathrm{C} \\
\mathrm{F} \\
\mathrm{C}\end{array}$ & $\begin{array}{l}\mathrm{R} \\
\mathrm{R} \\
\mathrm{R} \\
\mathrm{F} \\
\vdots \\
\vdots \\
\vdots \\
\vdots \\
:\end{array}$ & $\begin{array}{l}\text { R } \\
F \\
F \\
C \\
R \\
F \\
F \\
R \\
F \\
R \\
R \\
R\end{array}$ & $\begin{array}{ll}. & \mathrm{C} \\
: & \mathrm{C} \\
\dot{0} & \mathrm{C} \\
\mathrm{R} & \mathrm{C} \\
. & \mathrm{F} \\
: & \mathrm{C} \\
: & \mathrm{R} \\
& \mathrm{F} \\
. & \mathrm{R} \\
: & \mathrm{C} \\
. & \mathrm{R} \\
. & \mathrm{C}\end{array}$ & $\begin{array}{l}\text { C } \\
\text { C } \\
\text { C } \\
\text { C } \\
\text { F } \\
\text { C } \\
\text { F } \\
\text { C } \\
\text { F } \\
\text { C } \\
\text { F } \\
\text { C }\end{array}$ & $\begin{array}{l}\mathrm{R} \\
\mathrm{F} \\
\mathrm{F} \\
\mathrm{R} \\
\dot{\mathrm{R}} \\
\dot{\mathrm{R}} \\
\mathrm{R} \\
\dot{2} \\
\dot{\mathrm{R}}\end{array}$ & $\begin{array}{l}\mathrm{C} \\
\mathrm{F} \\
\mathrm{F} \\
\mathrm{F} \\
\mathrm{F} \\
\mathrm{F} \\
\mathrm{R} \\
\mathrm{F} \\
\mathrm{F} \\
\mathrm{F} \\
\mathrm{F} \\
\mathrm{F}\end{array}$ & $\begin{array}{ll}\mathrm{R} \\
\dot{\mathrm{R}} \\
\mathrm{R} \\
\mathrm{R} \\
\mathrm{F} \\
\mathrm{F} \\
\mathrm{R} \\
\mathrm{F} \\
\mathrm{R} \\
\mathrm{R} \\
\mathrm{R} \\
\mathrm{F} & \\
& \end{array}$ & $\begin{array}{l}\mathrm{F} \\
\mathrm{F} \\
\mathrm{C} \\
\mathrm{C} \\
\mathrm{C} \\
\mathrm{C} \\
\mathrm{C} \\
\mathrm{C} \\
\mathrm{C} \\
\mathrm{C} \\
\mathrm{F} \\
\mathrm{C}\end{array}$ & $\begin{array}{ll}F & \\
R & \\
R & C \\
C & C \\
F & C \\
F & C \\
R & C \\
R & C \\
R & C \\
R & C \\
R & C \\
F & C\end{array}$ & $\begin{array}{l}c \\
c \\
c \\
c \\
c \\
c \\
c \\
c \\
c \\
c \\
c \\
c\end{array}$ & $\begin{array}{ll}. & \mathrm{F} \\
\mathrm{R} & \mathrm{F} \\
\mathrm{R} & \mathrm{R} \\
\cdot & \mathrm{F} \\
\cdot & \mathrm{R} \\
& \mathrm{F}\end{array}$ & $\begin{array}{ll}\mathrm{F} & \\
\mathrm{F} & \mathrm{C} \\
\mathrm{R} & \mathrm{C} \\
\mathrm{F} & \mathrm{C} \\
\mathrm{R} & \mathrm{C} \\
\mathrm{F} & \mathrm{C} \\
\mathrm{i} & \mathrm{C} \\
\mathrm{R} & \mathrm{C} \\
\mathrm{R} & \mathrm{F} \\
\mathrm{R} & \mathrm{C} \\
\mathrm{R} & \mathrm{F} \\
\mathrm{F} & \mathrm{C}\end{array}$ & 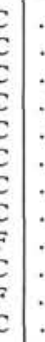 & $\begin{array}{l}\mathrm{R} \\
\\
\vdots \\
\vdots \\
\vdots \\
\vdots \\
\\
\\
\mathrm{R} \\
\mathrm{R} \\
\mathrm{R}\end{array}$ & : & $\begin{array}{l}\dot{C} \\
\text { C } \\
\text { C } \\
\text { C } \\
\text { R } \\
\vdots \\
\vdots \\
\vdots\end{array}$ & $\begin{array}{l}\mathrm{C} \\
\mathrm{F} \\
\mathrm{C} \\
\mathrm{C} \\
\mathrm{C} \\
\mathrm{C} \\
\mathrm{C} \\
\mathrm{F} \\
\mathrm{C} \\
\mathrm{C} \\
\mathrm{C} \\
\mathrm{C}\end{array}$ & $\begin{array}{l}\mathrm{F} \\
\mathrm{F} \\
\mathrm{R} \\
\mathrm{R} \\
\mathrm{R} \\
\dot{\mathrm{R}} \\
\mathrm{R} \\
\mathrm{R} \\
\mathrm{R} \\
\dot{\mathrm{R}}\end{array}$ & $\begin{array}{l}\mathrm{F} \\
\mathrm{F} \\
\mathrm{R} \\
\mathrm{R} \\
\mathrm{R} \\
\mathrm{R} \\
\dot{\mathrm{R}} \\
\mathrm{R} \\
\mathrm{R} \\
\mathrm{R} \\
\mathrm{R}\end{array}$ & $\dot{\mathrm{R}}$ & $\begin{array}{l}\mathrm{R} \\
\dot{\mathrm{R}} \\
\mathrm{F} \\
\dot{\mathrm{F}} \\
\dot{\mathrm{F}} \\
\dot{\mathrm{F}} \\
\mathrm{F} \\
\mathrm{R} \\
\mathrm{F}\end{array}$ & \begin{tabular}{l|l}
$\mathrm{C}$ & \\
$\mathrm{C}$ & \\
$\mathrm{C}$ & \\
$\mathrm{C}$ & \\
$\mathrm{C}$ & \\
$\mathrm{C}$ & \\
$\mathrm{C}$ & \\
$\mathrm{C}$ & \\
$\mathrm{C}$ & \\
$\mathrm{C}$ & \\
$\mathrm{F}$ & \\
$\mathrm{C}$ &
\end{tabular} & $\begin{array}{ll}\mathrm{C} & R \\
\mathrm{C} & \mathrm{R} \\
\mathrm{C} & \mathrm{R} \\
\mathrm{C} & \mathrm{C} \\
\mathrm{C} & \mathrm{R} \\
\mathrm{C} & \mathrm{R} \\
\mathrm{F} & \mathrm{C} \\
\mathrm{C} & \mathrm{R} \\
\mathrm{C} & \mathrm{C} \\
\mathrm{C} & \mathrm{R} \\
\mathrm{F} & \mathrm{R} \\
\mathrm{C} & \mathrm{C}\end{array}$ & $\begin{array}{ll}\mathrm{R} & \mathrm{R} \\
\mathrm{R} & \mathbf{R} \\
\mathrm{R} & \mathrm{R} \\
\mathrm{C} & \mathrm{C} \\
\mathrm{R} & \mathrm{R} \\
\mathrm{R} & \mathrm{R} \\
\mathrm{C} & \mathrm{C} \\
\mathrm{R} & \\
\mathrm{C} & \mathbf{C} \\
\mathrm{R} & \mathrm{F} \\
\mathrm{R} & \mathrm{F} \\
\mathrm{C} & \mathrm{C}\end{array}$ & $\begin{array}{ll}\mathrm{R} & \mathrm{C} \\
\mathrm{i} & \mathrm{C} \\
\mathrm{R} & \mathrm{C} \\
\mathrm{C} & \mathrm{C} \\
\mathrm{R} & \mathrm{C} \\
\mathrm{R} & \mathrm{F} \\
\mathrm{C} & \mathrm{C} \\
\dot{\mathrm{C}} & \mathrm{C} \\
\mathrm{C} & \mathrm{C} \\
\mathrm{F} & \mathrm{C} \\
\mathrm{F} & \mathrm{F}\end{array}$ & $\begin{array}{l}\mathrm{R} \\
\mathrm{R} \\
\text { : }\end{array}$ \\
\hline
\end{tabular}

$\mathbf{R}=$ Rare; $\mathbf{F}=$ Few; $\mathbf{C}=$ Common; $\mathbf{A}=$ Abundant; $\mathrm{VA}=$ Very abundant; ? = Questionably present; . = Not present. 
Table 3 (continued).

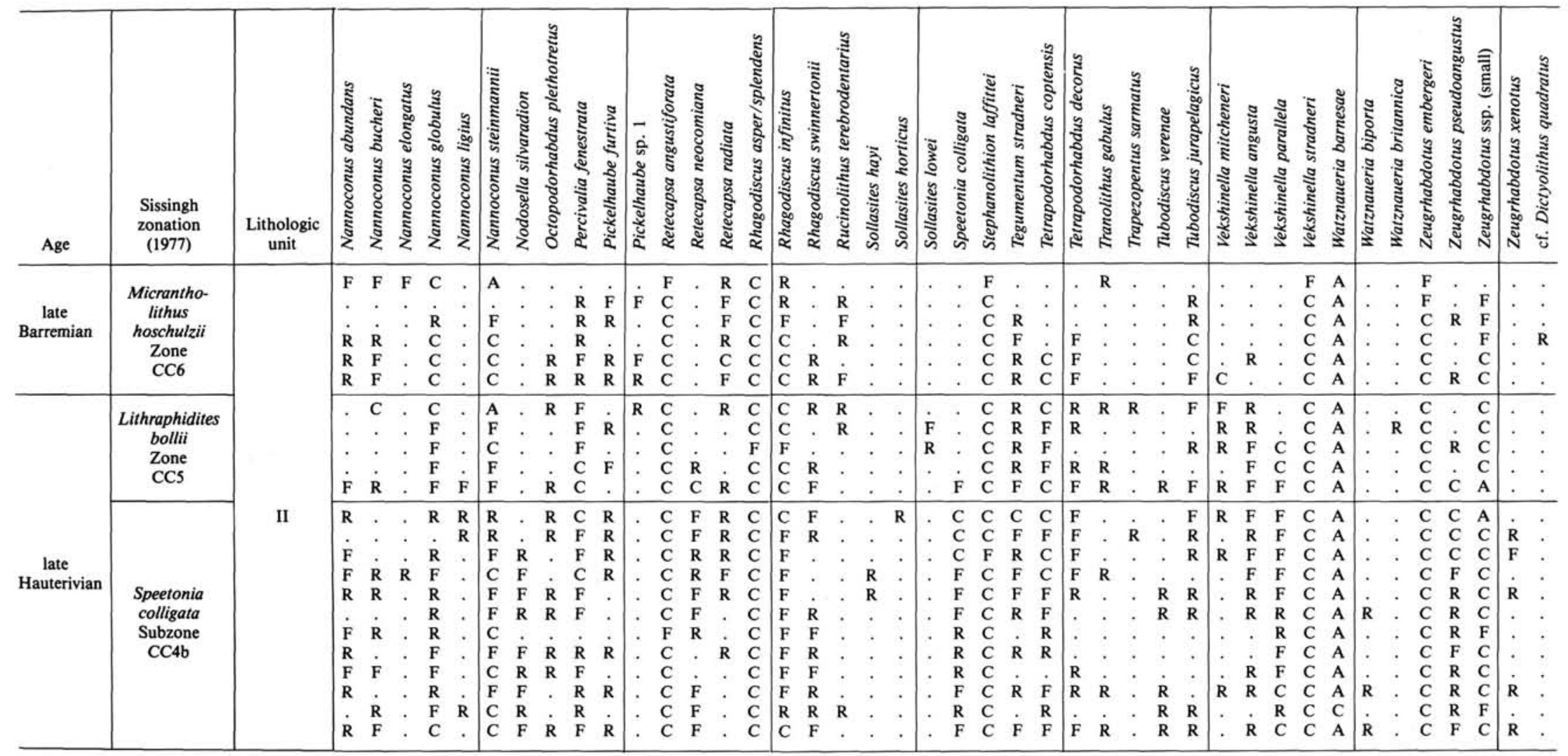


Table 4. Relative abundance of nannofossils, Hole 641C.

\begin{tabular}{|c|c|c|c|c|c|c|c|c|c|c|c|c|c|c|c|c|c|c|c|c|c|}
\hline Age & $\begin{array}{c}\text { Sissingh } \\
\text { zonation } \\
\text { (1977) }\end{array}$ & $\begin{array}{c}\text { Lithologic } \\
\text { unit }\end{array}$ & 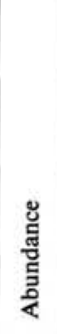 & 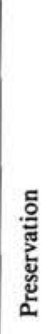 & Sample & 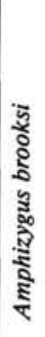 & 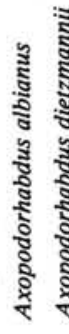 & 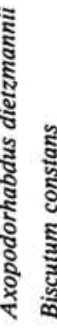 & 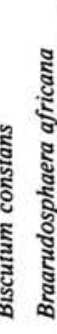 & 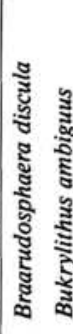 & 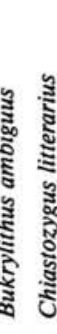 & 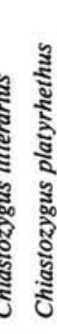 & 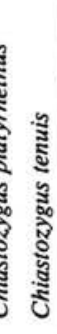 & 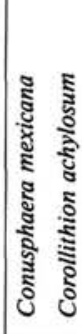 &  & 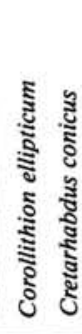 & 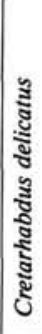 & 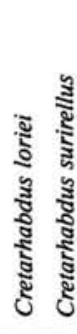 & 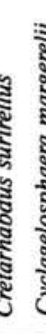 & 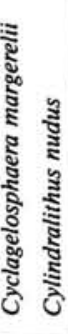 & 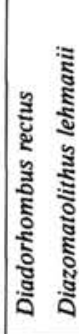 \\
\hline $\begin{array}{c}\text { early } \\
\text { Albian }\end{array}$ & $\begin{array}{c}\text { Braarudosphaera } \\
\text { Subzone } \\
\text { CC8a }\end{array}$ & III & $\begin{array}{c}\text { A } \\
\text { A } \\
\text { C } \\
\text { C } \\
\text { VA } \\
\text { F } \\
\text { F } \\
\text { C } \\
\text { C } \\
\text { A } \\
\text { C } \\
\text { F } \\
\text { A } \\
\text { A } \\
\text { A }\end{array}$ & $\begin{array}{l}\text { G } \\
\text { G } \\
\text { G } \\
\text { G } \\
\text { G } \\
\text { P } \\
\text { M } \\
\text { M } \\
\text { P } \\
\text { M } \\
\text { M } \\
\text { P } \\
\text { G } \\
\text { G } \\
\text { G }\end{array}$ & $\begin{array}{l}1 \mathrm{R}-1,52-53 \mathrm{~cm} \\
1 \mathrm{R}-1,120-121 \mathrm{~cm} \\
1 \mathrm{R}-2,85-86 \mathrm{~cm} \\
1 \mathrm{R}-3,81-82 \mathrm{~cm} \\
1 \mathrm{R}-4,17-18 \mathrm{~cm} \\
1 \mathrm{R}-6,39-40 \mathrm{~cm} \\
1 \mathrm{R}, \mathrm{CC} \\
2 \mathrm{R}-1,25-26 \mathrm{~cm} \\
2 \mathrm{R}-1,118-119 \mathrm{~cm} \\
3 \mathrm{R}-2,138 \mathrm{~cm} \\
3 \mathrm{R}-3,110 \mathrm{~cm} \\
6 \mathrm{R}-2,67 \mathrm{~cm} \\
6 \mathrm{R}-3,43 \mathrm{~cm} \\
6 \mathrm{R}-3,51 \mathrm{~cm} \\
6 \mathrm{R}-3,55 \mathrm{~cm}\end{array}$ & \begin{tabular}{|c}
$\mathbf{R}$ \\
$\mathbf{R}$ \\
$\mathbf{R}$ \\
$\mathbf{R}$ \\
$\mathbf{R}$ \\
$\vdots$ \\
$\vdots$ \\
$\dot{\mathrm{R}}$ \\
\\
\\
\\
$\mathbf{R}$ \\
\end{tabular} & $\begin{array}{ll}\mathrm{R} & \text { : } \\
: & \mathrm{R} \\
: & \mathrm{R} \\
: & \mathrm{R} \\
: & \mathrm{R} \\
: & : \\
: & : \\
: & : \\
: & : \\
: & : \\
: & \mathrm{R} \\
: & \mathrm{R} \\
& \mathrm{R} \\
\end{array}$ & $\begin{array}{ll}\dot{\text { R }} & \mathrm{C} \\
\mathrm{R} & \mathrm{C} \\
\mathrm{R} & \mathrm{C} \\
\mathrm{R} & \mathrm{C} \\
\text {. } & \mathrm{R} \\
\text {. } & \mathrm{R} \\
\text {. } & \mathrm{F} \\
\text {. } & \mathrm{F} \\
. & \mathrm{F} \\
. & \mathrm{F} \\
\dot{0} & \mathrm{R} \\
\mathrm{R} & \mathrm{C} \\
\mathrm{R} & \mathrm{C} \\
\mathrm{R} & \mathrm{C} \\
\end{array}$ & $\begin{array}{ll}C & \mathrm{R} \\
\mathrm{C} & : \\
\mathrm{C} & : \\
\mathrm{C} & : \\
\mathrm{C} & : \\
\mathrm{R} & : \\
\mathrm{R} & : \\
\mathrm{F} & : \\
\mathrm{F} & : \\
\mathrm{F} & : \\
\mathrm{F} & : \\
\mathrm{R} & : \\
\mathrm{C} & : \\
\mathrm{C} & :\end{array}$ & 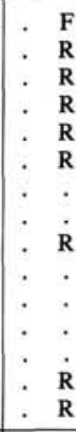 & 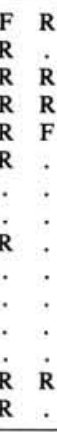 & $\begin{array}{l}\mathrm{R} \\
\dot{\mathrm{R}} \\
\mathrm{R}\end{array}$ & $\therefore$ & $\begin{array}{ll}. & \mathbf{R} \\
: & \mathbf{R} \\
: & \mathbf{R} \\
: & \mathbf{R} \\
: & \mathbf{F} \\
: & \mathbf{R} \\
: & : \\
: & : \\
: & \mathbf{R} \\
: & \mathbf{R} \\
: & . \\
: & \text { R } \\
: & \mathbf{R} \\
\end{array}$ & $\begin{array}{l}: \\
: \\
: \\
: \\
: \\
: \\
:\end{array}$ & 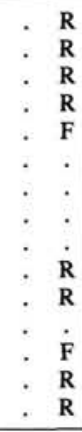 & $\begin{array}{l}: \\
: \\
: \\
: \\
: \\
: \\
: \\
.\end{array}$ & $\begin{array}{ll}\mathrm{R} & \mathrm{F} \\
: & \mathrm{F} \\
\mathrm{R} & \mathrm{F} \\
\mathrm{R} & \mathrm{F} \\
\mathrm{R} & \mathrm{F} \\
: & \mathrm{R} \\
: & \dot{\mathrm{R}} \\
: & \mathrm{R} \\
: & \mathrm{F} \\
: & \mathrm{F} \\
: & \dot{\mathrm{F}} \\
: & \mathrm{C} \\
: & \mathrm{C} \\
\end{array}$ & 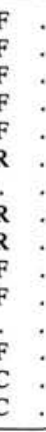 & 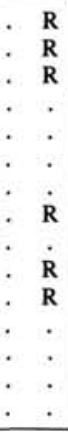 & $\begin{array}{ll}. & : \\
: & : \\
: & : \\
: & : \\
: & : \\
: & : \\
: & : \\
: & : \\
: & : \\
: & : \\
\end{array}$ \\
\hline $\begin{array}{c}\text { early } \\
\text { Albian } \\
\text { to } \\
\text { late } \\
\text { Aptian }\end{array}$ & $\begin{array}{c}\text { Rhagodiscus } \\
\text { angustus } \\
\text { Subzone } \\
\text { CC7b }\end{array}$ & IV & $\begin{array}{l}\text { B } \\
\text { C } \\
\text { A } \\
\text { A } \\
\text { A } \\
\text { VA } \\
\text { VA } \\
\text { VA } \\
\text { A } \\
\text { A } \\
\text { C } \\
\text { A } \\
\end{array}$ & $\begin{array}{l}\text { M } \\
\text { G } \\
\text { G } \\
\text { G } \\
\text { G } \\
\text { G } \\
\text { G } \\
\text { G } \\
\text { G } \\
M \\
M\end{array}$ & $\begin{array}{l}6 \mathrm{R}-3,77 \mathrm{~cm} \\
6 \mathrm{R}-3,78 \mathrm{~cm} \\
6 \mathrm{R}-3,91 \mathrm{~cm} \\
6 \mathrm{R}-3,123 \mathrm{~cm} \\
6 \mathrm{R}-4,8-9 \mathrm{~cm} \\
6 \mathrm{R}, \mathrm{CC} \\
7 \mathrm{R}-1,115-116 \mathrm{~cm} \\
7 \mathrm{R}-2,114-115 \mathrm{~cm} \\
8 \mathrm{R}-1,59-60 \mathrm{~cm} \\
8 \mathrm{R}-2,13-14 \mathrm{~cm} \\
8 \mathrm{R}-3,90-91 \mathrm{~cm} \\
8 \mathrm{R}-4,76-77 \mathrm{~cm} \\
\end{array}$ & $\begin{array}{l} \\
\dot{\mathrm{R}} \\
\dot{\mathrm{R}} \\
\dot{\mathrm{R}} \\
\mathrm{R} \\
\mathrm{R} \\
\mathrm{R} \\
\mathrm{R}\end{array}$ & 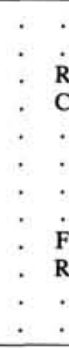 & $\begin{array}{ll}\cdot & \dot{C} \\
\dot{R} & \mathrm{C} \\
\mathrm{C} & \mathrm{C} \\
\cdot & \mathrm{C} \\
\cdot & \mathrm{C} \\
\cdot & \mathrm{C} \\
\dot{0} & \mathrm{C} \\
\mathrm{F} & \mathrm{A} \\
\mathrm{R} & \mathrm{C} \\
\cdot & \mathrm{C} \\
\cdot & \mathrm{F} \\
\end{array}$ & $\begin{array}{l:}\dot{C} \\
\mathrm{C}\end{array}:$ & 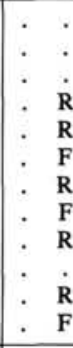 & $\begin{array}{ll}\cdot & \text {. } \\
\cdot & \text {. } \\
\dot{R} & \dot{\mathrm{C}} \\
\mathrm{R} & \\
\mathrm{F} & \mathrm{R} \\
\mathrm{R} & \mathrm{R} \\
\mathrm{F} & \mathrm{F} \\
\mathrm{R} & \mathrm{F} \\
\dot{\mathrm{R}} & \mathrm{R} \\
\mathrm{R} & \mathrm{R} \\
\mathrm{F} & \mathrm{R} \\
\end{array}$ & 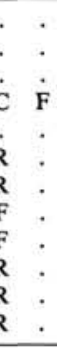 & $\therefore$. & 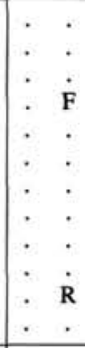 & $\begin{array}{l}: \\
: \\
: \\
: \\
: \\
: \\
.\end{array}$ & $\begin{array}{ll}: & \text { R } \\
: & \mathrm{F} \\
: & \mathrm{C} \\
: & \mathrm{F} \\
: & \mathrm{F} \\
: & \mathrm{F} \\
: & \mathrm{C} \\
: & \mathrm{C} \\
: & \mathrm{R} \\
: & \text {. } \\
\end{array}$ & $\begin{array}{l}: \\
: \\
\dot{.} \\
\dot{.} \\
: \\
. \\
. \\
.\end{array}$ & $\begin{array}{ll}: & \dot{\mathrm{F}} \\
\dot{0} & \mathrm{~F} \\
\mathrm{~F} & \mathrm{C} \\
: & \mathrm{F} \\
\dot{\mathrm{R}} & \mathrm{F} \\
. & \mathrm{F} \\
\dot{0} & \mathrm{~F} \\
. & \mathrm{F} \\
. & \mathrm{R} \\
. & \mathrm{R} \\
\end{array}$ & 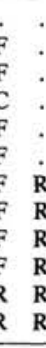 & $\begin{array}{ll}: & : \\
: & : \\
: & : \\
: & : \\
\\
\end{array}$ & 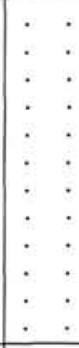 \\
\hline $\begin{array}{c}\text { early } \\
\text { Aptian }\end{array}$ & $\begin{array}{c}\text { Hayesites } \\
\text { irregularis } \\
\text { Subzone } \\
\text { CC7a }\end{array}$ & v & $\begin{array}{l}\text { C } \\
\text { VA } \\
\text { VA } \\
\text { A } \\
\text { A } \\
\text { A } \\
\text { A } \\
\text { A } \\
\text { A } \\
\text { A } \\
\text { A } \\
\text { A } \\
\text { A } \\
\text { A }\end{array}$ & $\begin{array}{l}\text { M } \\
\text { G } \\
\text { G } \\
\text { M } \\
\text { M } \\
\text { M } \\
\text { G } \\
\text { M } \\
\text { P } \\
\text { G } \\
\text { G } \\
\text { G } \\
\text { M } \\
\text { P }\end{array}$ & $\begin{array}{l}8 \mathrm{R}-4,121-122 \mathrm{~cm} \\
9 \mathrm{R}-1,57-58 \mathrm{~cm} \\
9 \mathrm{R}-3,139 \mathrm{~cm} \\
9 \mathrm{R}-4,37-38 \mathrm{~cm} \\
9 \mathrm{R}, \mathrm{CC} \\
10 \mathrm{R}-2,56 \mathrm{~cm} \\
10 \mathrm{R}-3,133 \mathrm{~cm} \\
10 \mathrm{R}-4,15-16 \mathrm{~cm} \\
10 \mathrm{R}-4,46 \mathrm{~cm} \\
10 \mathrm{R}, \mathrm{CC} \\
11 \mathrm{R}-2,55-56 \mathrm{~cm} \\
11 \mathrm{R}-2,60 \mathrm{~cm} \\
11 \mathrm{R}-2,108 \mathrm{~cm} \\
11 \mathrm{R}-3,76 \mathrm{~cm} \\
\end{array}$ & $\begin{array}{l}\mathrm{R} \\
\dot{\mathrm{F}} \\
\mathrm{F} \\
\mathrm{R} \\
\mathrm{R} \\
\dot{\mathrm{R}} \\
\dot{\mathrm{R}} \\
\mathrm{R} \\
\mathrm{F} \\
\end{array}$ & 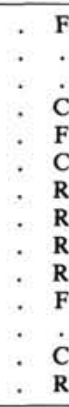 & $\begin{array}{ll}\mathrm{F} & \text {. } \\
\cdot & \mathrm{C} \\
\dot{0} & \mathrm{C} \\
\mathrm{C} & \mathrm{C} \\
\mathrm{F} & \mathrm{C} \\
\mathrm{C} & \mathrm{C} \\
\mathrm{R} & \mathrm{C} \\
\mathrm{R} & \mathrm{C} \\
\mathrm{R} & \mathrm{C} \\
\mathrm{R} & \mathrm{F} \\
\mathrm{F} & \mathrm{C} \\
\dot{\mathrm{C}} & \mathrm{C} \\
\mathrm{C} & \mathrm{C} \\
\mathrm{R} & \mathrm{C} \\
\end{array}$ &  & 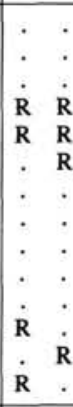 & 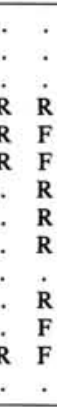 & $\begin{array}{l}: \\
: \\
: \\
: \\
: \\
: \\
: \\
:\end{array}$ & $\begin{array}{l}\text {. } \\
: \\
: \\
: \\
: \\
:\end{array}$ & 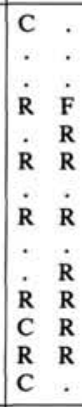 & $\begin{array}{c}\dot{\cdot} \\
\dot{\mathrm{C}} \\
\mathrm{F} \\
\mathrm{R} \\
\dot{\mathrm{R}} \\
\dot{\cdot} \\
\dot{\mathrm{R}} \\
\mathrm{R} \\
\mathrm{R} \\
\dot{ }\end{array}$ & 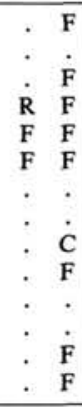 & $\begin{array}{l}\dot{.} \\
\dot{\mathrm{R}} \\
\mathrm{R} \\
\dot{ } \\
\dot{\mathrm{R}} \\
\mathrm{R} \\
\mathrm{F} \\
\mathrm{R} \\
\mathrm{R} \\
\mathrm{R} \\
\end{array}$ & $\begin{array}{cc} & \mathrm{C} \\
\mathbf{R} & \mathrm{F} \\
\dot{\mathrm{R}} & \mathrm{F} \\
. & \mathrm{F} \\
. & \mathrm{F} \\
. & \mathrm{R} \\
\dot{0} & \mathrm{R} \\
\dot{\mathrm{R}} & \mathrm{F} \\
. & \mathrm{F} \\
. & \mathrm{F} \\
\dot{0} & \mathrm{~F} \\
\mathbf{R} & \mathrm{F} \\
. & \mathrm{R} \\
\end{array}$ & $\begin{array}{l}\mathrm{C} \\
\mathrm{R} \\
\mathrm{C} \\
\mathrm{C} \\
\mathrm{C} \\
\mathrm{C} \\
\mathrm{C} \\
\mathrm{F} \\
\mathrm{C} \\
\mathrm{F} \\
\mathrm{C} \\
\mathrm{C} \\
\mathrm{C} \\
\mathrm{F}\end{array}$ & $\begin{array}{ll}\mathrm{C} & : \\
\mathrm{R} & : \\
\mathrm{C} & : \\
\mathrm{C} & : \\
\mathrm{C} & : \\
\mathrm{C} & : \\
\mathrm{C} & : \\
\mathrm{F} & : \\
\mathrm{C} & : \\
\mathrm{F} & : \\
\mathrm{C} & : \\
\mathrm{C} & : \\
\mathrm{C} & : \\
\mathrm{F} & : \\
\end{array}$ & $\begin{array}{ll} & \vdots \\
\mathbf{R} & \vdots \\
\vdots & \vdots \\
\vdots & \vdots \\
\vdots & \vdots \\
\vdots & \vdots \\
\vdots & \vdots \\
& \vdots \\
\end{array}$ \\
\hline $\begin{array}{c}\text { late } \\
\text { Barremian }\end{array}$ & $\begin{array}{c}\text { Micrantho- } \\
\text { lithus } \\
\text { hoschulzii } \\
\text { Zone } \\
\text { CC6 }\end{array}$ & VI & $\begin{array}{l}\text { A } \\
\text { A } \\
\text { VA } \\
\text { A } \\
\text { A } \\
\text { A } \\
\text { A } \\
\text { C } \\
\text { A } \\
\text { C } \\
\text { VA } \\
\text { A } \\
\text { A } \\
\text { A } \\
\text { A } \\
\text { A } \\
\text { A } \\
\text { A } \\
\text { A } \\
\text { A }\end{array}$ & 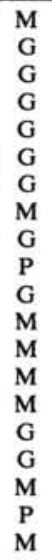 & $\begin{array}{l}12 \mathrm{R}-1,27-28 \mathrm{~cm} \\
12 \mathrm{R}-2,44 \mathrm{~cm} \\
12 \mathrm{R}-3,103 \mathrm{~cm} \\
12 \mathrm{R}-3,107 \mathrm{~cm} \\
12 \mathrm{R}-4,91 \mathrm{~cm} \\
12 \mathrm{R}-5,96 \mathrm{~cm} \\
12 \mathrm{R}-6,4-5 \mathrm{~cm} \\
13 \mathrm{R}-1,45-46 \mathrm{~cm} \\
13 \mathrm{R}-2,36 \mathrm{~cm} \\
13 \mathrm{R}-4,119 \mathrm{~cm} \\
13 \mathrm{R}-5,27 \mathrm{~cm} \\
14 \mathrm{R}-3,46 \mathrm{~cm} \\
14 \mathrm{R}-4,31 \mathrm{~cm} \\
15 \mathrm{R}-2,40 \mathrm{~cm} \\
15 \mathrm{R}-3,27 \mathrm{~cm} \\
15 \mathrm{R}-5,40 \mathrm{~cm} \\
16 \mathrm{R}-2,35 \mathrm{~cm} \\
16 \mathrm{R}-4,61 \mathrm{~cm} \\
16 \mathrm{R}-5,36 \mathrm{~cm} \\
16 \mathrm{R}-8,28 \mathrm{~cm}\end{array}$ & $\begin{array}{l}: \\
: \\
: \\
: \\
: \\
: \\
: \\
: \\
: \\
: \\
:\end{array}$ & $\begin{array}{ll}: & \mathrm{R} \\
: & \mathrm{R} \\
: & \mathrm{R} \\
: & \mathrm{F} \\
: & \mathrm{F} \\
: & \mathrm{F} \\
: & \mathrm{F} \\
: & \mathrm{F} \\
: & \mathrm{R} \\
: & \mathrm{R} \\
: & \mathrm{R} \\
: & \mathrm{R} \\
: & \text { R } \\
: & \text { R }\end{array}$ & $\begin{array}{ll}R & F \\
R & F \\
R & C \\
F & C \\
F & C \\
F & C \\
F & C \\
\text { F } & \text { C } \\
\text { F } & C \\
\text { F } & C \\
R & C \\
R & C \\
R & C \\
R & C \\
R & F \\
\text {. } & C \\
R & F \\
\text { R } & \text { F } \\
\text { R }\end{array}$ & 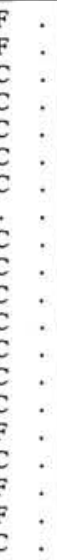 & 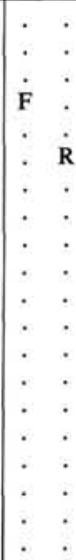 & 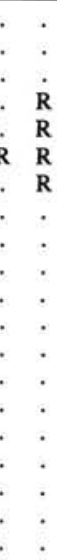 & $\begin{array}{l}\text { R } \\
\vdots \\
\vdots \\
\vdots \\
\vdots \\
:\end{array}$ & $\begin{array}{l}: \\
: \\
\vdots \\
: \\
\dot{\mathrm{R}} \\
: \\
: \\
: \\
\dot{\mathrm{R}} \\
:\end{array}$ & 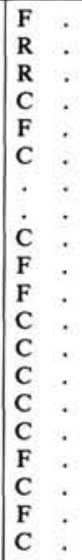 & $\begin{array}{l}: \\
: \\
: \\
: \\
: \\
: \\
: \\
:\end{array}$ & $\begin{array}{ll}: & \mathrm{F} \\
: & \mathrm{F} \\
: & \mathrm{F} \\
: & \mathrm{F} \\
: & \mathrm{C} \\
: & \mathrm{F} \\
: & \mathrm{F} \\
: & \mathrm{F} \\
: & \mathrm{C} \\
: & \mathrm{F} \\
: & \mathrm{F} \\
: & \mathrm{C} \\
: & \mathrm{C} \\
: & \mathrm{F}\end{array}$ & R & $\begin{array}{cc}. & \mathrm{F} \\
: & \mathrm{R} \\
\mathrm{R} & \mathrm{F} \\
. & \mathrm{F} \\
: & \mathrm{F} \\
: & \mathrm{C} \\
: & \mathrm{F} \\
: & \text { F } \\
: & \mathrm{R} \\
: & \mathrm{F} \\
: & \mathrm{F} \\
: & \mathrm{R} \\
: & \mathrm{F} \\
: & \mathrm{R} \\
. & \dot{\mathrm{F}} \\
. & \mathrm{F} \\
: & \mathrm{R} \\
. & \mathrm{F}\end{array}$ & $\begin{array}{l}\mathrm{R} \\
\mathrm{R} \\
\mathrm{C} \\
\mathrm{C} \\
\mathrm{C} \\
\mathrm{C} \\
\mathrm{C} \\
\mathrm{C} \\
\mathrm{C} \\
\mathrm{C} \\
\mathrm{C} \\
\mathrm{C} \\
\mathrm{C} \\
\mathrm{F} \\
\mathrm{R} \\
\mathrm{C} \\
\mathrm{F} \\
\mathrm{C} \\
\mathrm{C}\end{array}$ & 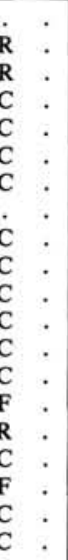 & 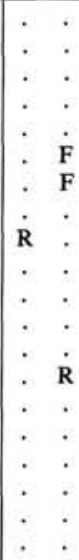 \\
\hline
\end{tabular}

$\mathrm{R}=$ Rare; $\mathrm{F}=$ Few $\mathrm{C}=$ Common; $\mathrm{A}=$ Abundant; $\mathrm{VA}=$ Very abundant; $?=$ Questionably present; . = Not present. 


\begin{tabular}{|c|c|c|c|c|c|c|c|c|c|c|c|c|c|c|c|c|c|c|c|c|c|c|c|c|c|c|c|c|c|c|c|c|}
\hline 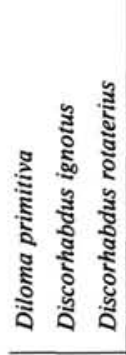 & & 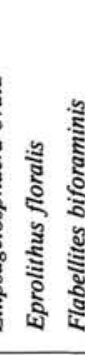 & & 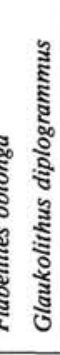 & &  & & & & 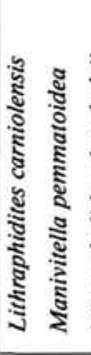 & & & & 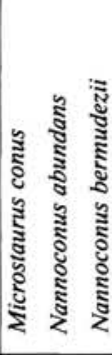 & & & & 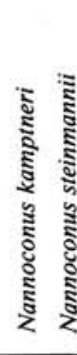 & & & & 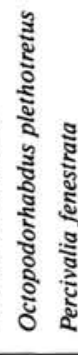 & & 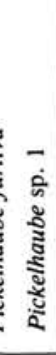 & & 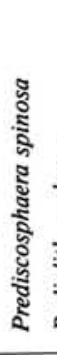 & & 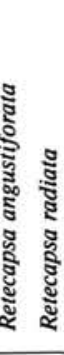 & & 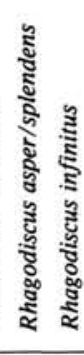 & & 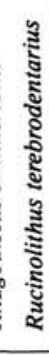 \\
\hline $\begin{array}{ll}\mathrm{C} & \mathrm{F} \\
\mathrm{C} & \mathrm{F} \\
\mathrm{C} & \mathrm{R} \\
\mathrm{F} & \mathrm{R} \\
\mathrm{C} & \mathrm{F} \\
\mathrm{R} & \mathrm{R} \\
\mathrm{R} & \mathrm{R} \\
\mathrm{F} & \mathrm{R} \\
\mathrm{F} & \mathrm{R} \\
\mathrm{F} & \mathrm{C} \\
\mathrm{F} & \mathrm{F} \\
\dot{\mathrm{C}} & \\
\mathrm{C} & \mathrm{R} \\
\mathrm{C} & \mathrm{R} \\
\end{array}$ & & $\begin{array}{cc}F & R \\
R & R \\
R & R \\
R & R \\
R & . \\
R & \\
& \\
R & \\
R & R \\
F & F \\
F & F \\
\dot{F} & F \\
R & F \\
C & F \\
\end{array}$ & $\begin{array}{l}\mathrm{R} \\
\mathrm{R} \\
\mathrm{R} \\
\mathrm{R} \\
\mathrm{R} \\
\vdots \\
\vdots \\
\mathrm{R}\end{array}$ & $\begin{array}{ll} & \mathrm{F} \\
0 & \mathrm{C} \\
\mathrm{F} & \mathrm{C} \\
\mathrm{C} & \mathrm{C} \\
\mathrm{R} \\
\mathrm{F} \\
\mathrm{F} \\
\mathrm{F} \\
\mathrm{F} \\
\mathrm{F} \\
\mathrm{F} \\
\mathrm{F} \\
& \mathrm{C} \\
\mathrm{F} & \mathrm{F} \\
\end{array}$ & $\begin{array}{l}\text { R } \\
\text { R. } \\
\text { R } \\
\text {. }\end{array}$ & 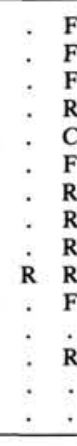 & 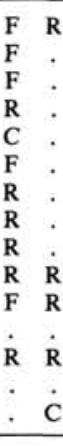 & $\begin{array}{l}\text { R } \\
\vdots \\
\vdots \\
\vdots \\
\vdots\end{array}$ & & $\begin{array}{ll}\mathrm{A} & \mathrm{F} \\
\mathrm{C} & \mathrm{C} \\
\mathrm{F} & \mathrm{F} \\
\mathrm{C} & \mathrm{F} \\
\mathrm{A} & \mathrm{C} \\
\mathrm{R} & \mathrm{R} \\
\mathrm{R} & \mathrm{R} \\
\mathrm{R} & \mathrm{R} \\
\mathrm{F} & \mathrm{F} \\
\mathrm{C} & \mathrm{F} \\
\mathrm{C} & \mathrm{F} \\
\text { C } & \mathrm{R} \\
\mathrm{C} & \mathrm{C} \\
\mathrm{C} & \mathrm{F} \\
\mathrm{C} & \mathrm{F} \\
\end{array}$ & . & & & & & & & & & & & & & & $\mathrm{R}$ & $\begin{array}{l}\mathrm{R} \\
\dot{\mathrm{R}} \\
\dot{\mathrm{F}} \\
\dot{0} \\
\vdots \\
\dot{\mathrm{R}} \\
\vdots \\
\vdots \\
\end{array}$ & $\begin{array}{l}\vdots \\
\vdots \\
\vdots \\
\vdots \\
\mathrm{R} \\
\\
\mathrm{R} \\
\mathrm{R} \\
\mathrm{R} \\
\end{array}$ & $\begin{array}{l}C \\
C \\
F \\
F \\
C \\
R \\
R\end{array}$ & & $\begin{array}{ll}\mathrm{C} & \mathrm{F} \\
\mathrm{C} & \mathrm{F} \\
\mathrm{F} & \mathrm{R} \\
\mathrm{F} & \\
\mathrm{C} & \dot{\mathrm{R}} \\
\mathrm{R} & \mathrm{R} \\
\dot{L} & \dot{\mathrm{F}} \\
\mathrm{R} & \mathrm{R} \\
\mathrm{C} & \mathrm{R} \\
\mathrm{C} & \mathrm{R} \\
\mathrm{R} & \dot{\mathrm{C}} \\
\mathrm{C} & \mathrm{R} \\
\mathrm{C} & \mathrm{F} \\
\mathrm{C} & \mathrm{F} \\
\end{array}$ & $\begin{array}{c}C \\
C \\
R \\
F \\
C \\
C \\
\text { R } \\
R \\
R \\
R \\
\vdots \\
\vdots \\
\end{array}$ & \\
\hline $\begin{array}{cc}\dot{C} & \dot{\mathrm{F}} \\
\dot{C} & \mathrm{~F} \\
\mathrm{C} & \mathrm{F} \\
\mathrm{C} & \dot{\mathrm{R}} \\
\mathrm{C} & \dot{ } \\
\mathrm{C} & \mathrm{R} \\
\mathrm{A} & \dot{\mathrm{C}} \\
\mathrm{C} & \mathrm{R} \\
\mathrm{F} & \mathrm{C} \\
\mathrm{R} & \mathrm{F} \\
\end{array}$ & & $\begin{array}{ll}\mathrm{F} & \mathrm{R} \\
\mathrm{R} & \\
\mathrm{C} & \mathrm{F} \\
\mathrm{F} & \mathrm{R} \\
\mathrm{C} & \mathrm{R} \\
\mathrm{C} & \mathrm{R} \\
\mathrm{F} & \mathrm{F} \\
\mathrm{C} & \mathrm{R} \\
\mathrm{F} & \\
\mathrm{R} & \\
\mathrm{R} & \mathrm{R} \\
\end{array}$ & $\begin{array}{l:}\mathrm{R} \\
\dot{\mathrm{F}}\end{array}$ & $\begin{array}{l}\mathrm{C}^{\mathrm{C}} \\
\mathrm{C} \\
\mathrm{C} \\
\mathrm{C} \\
\mathrm{C} \\
\mathrm{C} \\
\mathrm{C} \\
\mathrm{C} \\
\mathrm{C} \\
\mathrm{C} \\
\mathrm{C} \\
\mathrm{C} \\
\mathrm{C} \\
\mathrm{F} \\
\mathrm{F}\end{array}$ & & 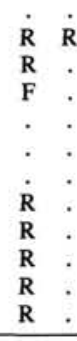 & 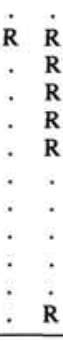 & 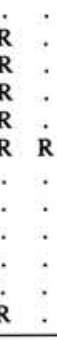 & & $\begin{array}{ll}\mathrm{V} & \mathrm{C} \\
\mathrm{C} & \mathrm{F} \\
\mathrm{A} & \mathrm{F} \\
\mathrm{C} & \mathrm{C} \\
\mathrm{C} & \mathrm{F} \\
\mathrm{C} & \mathrm{F} \\
\mathrm{C} & \mathrm{F} \\
\mathrm{A} & \mathrm{C} \\
\mathrm{C} & \mathrm{F} \\
\end{array}$ & $\begin{array}{l}\dot{R} \\
\mathrm{R}\end{array}$ & $\mathrm{K}$ & & : & $\mathrm{K}$ & & & 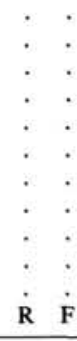 & $\mathbf{F}$ & & & $\begin{array}{ll}\vdots & \vdots \\
\vdots & { }^{\mathrm{R}} \\
\vdots & \vdots \\
\vdots & \\
\vdots & \mathrm{R} \\
\vdots & \mathrm{R} \\
\vdots & \mathrm{R} \\
\end{array}$ & 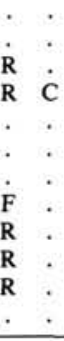 & & & $\begin{array}{l}: \\
: \\
: \\
: \\
:\end{array}$ & & R & & $\begin{array}{ll}\mathrm{F} & \mathrm{R} \\
\mathrm{C} & \mathrm{R} \\
\mathrm{C} & \mathrm{C} \\
\mathrm{F} & \mathrm{F} \\
\mathrm{C} & \mathrm{F} \\
\mathrm{F} & \mathrm{F} \\
\mathrm{C} & \mathrm{C} \\
\mathrm{A} & \mathrm{C} \\
\mathrm{C} & \mathrm{F} \\
\mathrm{C} & \mathrm{F} \\
\mathrm{C} & \mathrm{F} \\
\end{array}$ & $\begin{array}{l}\vdots \\
\vdots \\
\vdots\end{array}$ & - \\
\hline 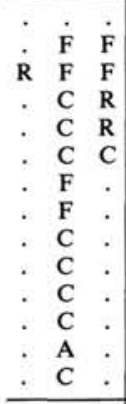 & & $\begin{array}{ll}: & \mathrm{R} \\
\vdots & \mathrm{R} \\
\vdots & \mathrm{R} \\
\vdots & \mathrm{R} \\
\vdots & \mathrm{F} \\
\vdots & \vdots \\
\vdots & \mathrm{R} \\
\vdots & \mathrm{R} \\
\vdots & \mathrm{R} \\
\end{array}$ & $\begin{array}{l}\mathrm{R} \\
\mathrm{R} \\
\mathrm{R} \\
\mathrm{R} \\
\mathrm{R}\end{array}$ & $\begin{array}{l}\mathrm{F} \\
\mathrm{O} \\
\mathrm{O} \\
\mathrm{R} \\
\mathrm{F} \\
\mathrm{C} \\
\mathrm{C} \\
\mathrm{C} \\
\mathrm{C} \\
\mathrm{C} \\
\mathrm{C} \\
\mathrm{C} \\
\mathrm{C} \\
\mathrm{C} \\
\mathrm{C} \\
\mathrm{C} \\
\mathrm{F} \\
\mathrm{C}\end{array}$ & & $\begin{array}{l:}\text { R } \\
\text { F } \\
\text { R } \\
\text { R } \\
\text { R } \\
\text { R } \\
\text { R } \\
\dot{R}\end{array}$ & : . & . . & & $\begin{array}{l}\mathrm{C} \\
\mathrm{F} \\
\mathrm{C} \\
\mathrm{C} \\
\mathrm{C} \\
\mathrm{C} \\
\mathrm{R} \\
\dot{C} \\
\mathrm{C} \\
\mathrm{C} \\
\mathrm{F} \\
\dot{\mathrm{R}} \\
\mathrm{R} \\
\mathrm{F}\end{array}$ & $\begin{array}{c}\mathrm{A} \\
\vdots \\
\dot{\mathrm{R}} \\
\mathrm{R} \\
\mathrm{R} \\
\dot{0} \\
\vdots \\
\vdots \\
\dot{\mathrm{C}} \\
\dot{\mathrm{C}}\end{array}$ & : & & 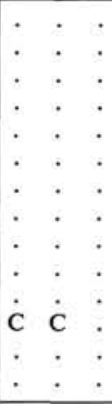 & $\begin{array}{l}\mathrm{R} \\
: \\
:\end{array}$ & & ? & $\begin{array}{ll} & \mathrm{C} \\
\dot{\mathrm{R}} & \mathrm{C} \\
\vdots & \mathrm{F} \\
\vdots & \mathrm{F} \\
\vdots & \mathrm{R} \\
\vdots & \mathrm{R} \\
\vdots & \vdots \\
\vdots & \mathrm{R} \\
\vdots & \mathrm{C} \\
\vdots & \mathrm{R} \\
\end{array}$ & $\begin{array}{l}\mathrm{C} \\
\dot{\mathrm{F}} \\
\mathrm{F} \\
\mathrm{R} \\
\mathrm{R}\end{array}$ & & : & $\begin{array}{ll}\vdots & \\
\vdots & \\
\vdots & \mathrm{F} \\
\dot{\mathrm{R}} & \mathrm{F} \\
\vdots & \mathrm{F} \\
\dot{\mathrm{R}} & \\
\vdots & \dot{\mathrm{R}} \\
\vdots & \dot{\mathrm{F}} \\
\dot{\mathrm{F}} & \mathrm{F}\end{array}$ & 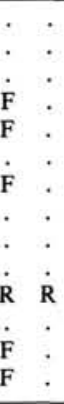 & $\begin{array}{l}\vdots \\
\vdots \\
\vdots \\
\mathrm{R} \\
\vdots \\
\vdots\end{array}$ & & . & 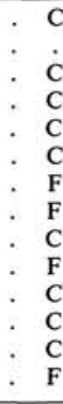 & & & $\begin{array}{ll}\mathrm{C} & \mathrm{C} \\
\mathrm{C} & \mathrm{F} \\
\mathrm{F} & \mathrm{C} \\
\mathrm{C} & \mathrm{C} \\
\mathrm{C} & \mathrm{C} \\
\mathrm{C} & \mathrm{C} \\
\mathrm{C} & \mathrm{R} \\
\mathrm{F} & \mathrm{F} \\
\mathrm{C} & \mathrm{C} \\
\mathrm{F} & \mathrm{F} \\
\mathrm{C} & \mathrm{F} \\
\mathrm{C} & \mathrm{F} \\
\mathrm{F} & \mathrm{C} \\
\mathrm{C} & \mathrm{R}\end{array}$ & $\begin{array}{l}\dot{\mathrm{F}} \\
\vdots \\
\vdots \\
\vdots\end{array}$ & . \\
\hline 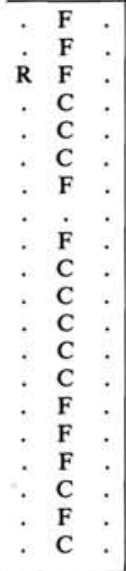 & $\begin{array}{l}\mathrm{K} \\
\mathrm{R}\end{array}$ & 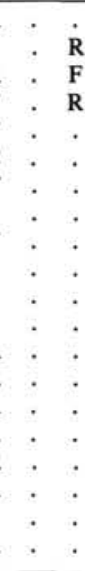 & $\begin{array}{l}: \\
\vdots \\
\vdots\end{array}$ & $\begin{array}{l}\mathrm{C} \\
\mathrm{F} \\
\mathrm{C} \\
\mathrm{C} \\
\mathrm{F} \\
\mathrm{F} \\
\mathrm{F} \\
\mathrm{F} \\
\mathrm{C} \\
\mathrm{C} \\
\mathrm{C} \\
\mathrm{C} \\
\mathrm{C}\end{array}$ & & $\begin{array}{c:}\dot{\mathrm{F}} \\
\dot{\mathrm{F}} \\
\dot{\mathrm{F}}\end{array}$ & : & & & $\begin{array}{l}\text { C } \\
\text { F } \\
\text { C } \\
\text { F } \\
\text { F } \\
\text { C } \\
\text { C } \\
\text { C } \\
\text { F } \\
\text { C } \\
\text { F } \\
\text { C }\end{array}$ & $\begin{array}{l}\text { F } \\
\text { C } \\
\text { R } \\
\text { F } \\
\vdots \\
\text { C } \\
\text { F } \\
\text { F } \\
\text { F } \\
\text { F } \\
\text { C }\end{array}$ & & & 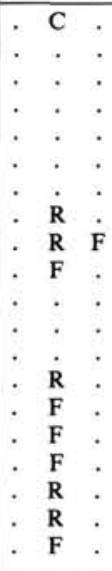 & $\begin{array}{l} \\
\dot{\mathrm{F}} \\
\mathrm{F}\end{array}$ & & & 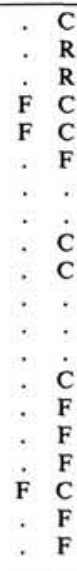 & $\begin{array}{l}\dot{\mathrm{C}} \\
\mathrm{C} \\
\dot{\mathrm{C}} \\
\dot{\mathrm{C}} \\
\mathrm{F} \\
\mathrm{F} \\
\mathrm{F} \\
\mathrm{C}\end{array}$ & & & $\begin{array}{ll}: & \mathrm{R} \\
: & \mathrm{F} \\
: & \mathrm{F} \\
: & \mathrm{R} \\
: & \mathrm{F} \\
: & \mathrm{F} \\
: & \mathrm{F} \\
\vdots & \mathrm{F} \\
: & \mathrm{F} \\
\vdots & \mathrm{F} \\
: & \mathrm{R} \\
: & \mathrm{F} \\
: & \mathrm{F}\end{array}$ & $\begin{array}{ll}\mathrm{F} & \dot{\mathrm{R}} \\
\mathrm{R} & \\
\mathrm{F} & \vdots \\
\mathrm{F} & \vdots \\
\mathrm{F} & \vdots \\
\mathrm{F} & \vdots \\
\mathrm{R} & \vdots \\
\mathrm{R} & \vdots \\
\mathrm{F} & \vdots \\
\mathrm{F} & \vdots \\
\mathrm{R} & \end{array}$ & $\begin{array}{l} \\
\vdots \\
\vdots \\
\vdots \\
\vdots \\
\vdots \\
\vdots \\
\vdots\end{array}$ & & . & : & 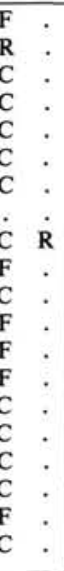 & & $\begin{array}{ll}\mathrm{C} & \mathrm{C} \\
\mathrm{C} & \mathrm{F} \\
\mathrm{C} & \mathrm{F} \\
\mathrm{C} & \mathrm{R} \\
\mathrm{C} & \mathrm{F} \\
\mathrm{C} & \mathrm{F} \\
\mathrm{C} & \mathrm{F} \\
\mathrm{C} & \mathrm{R} \\
\mathrm{C} & \mathrm{F} \\
\mathrm{C} & \mathrm{F} \\
\mathrm{C} & \mathrm{F} \\
\mathrm{C} & \mathrm{F}\end{array}$ & $:$ & 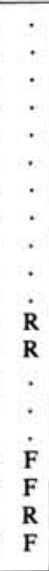 \\
\hline
\end{tabular}


Table 4 (continued).

\begin{tabular}{|c|c|c|c|c|c|c|c|c|c|c|c|c|c|c|c|c|c|c|c|c|}
\hline Age & $\begin{array}{c}\text { Sissingh } \\
\text { zonation } \\
\text { (1977) }\end{array}$ & $\begin{array}{l}\text { Lithologic } \\
\text { unit }\end{array}$ & 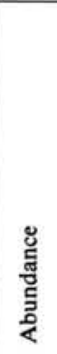 & 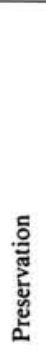 & Sample & 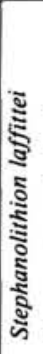 & 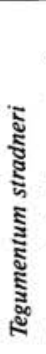 & 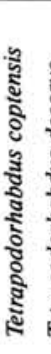 & 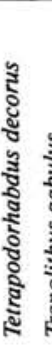 & 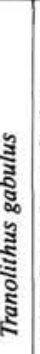 & 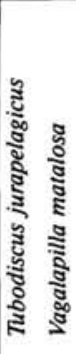 & 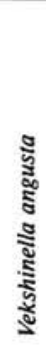 &  & 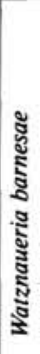 & 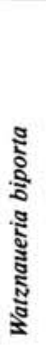 & 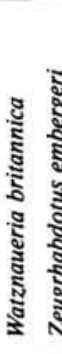 & 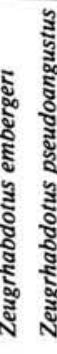 & & & 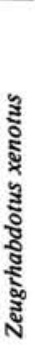 \\
\hline $\begin{array}{c}\text { early } \\
\text { Albian }\end{array}$ & $\begin{array}{c}\text { Braarudosphaera } \\
\text { Subzone } \\
\text { CC8a }\end{array}$ & III & $\begin{array}{l}\text { A } \\
\text { A } \\
\text { C } \\
\text { C } \\
\text { VA } \\
\text { F } \\
\text { F } \\
\text { C } \\
\text { C } \\
\text { A } \\
\text { C } \\
\text { F } \\
\text { A } \\
\text { A } \\
\text { A }\end{array}$ & $\begin{array}{l}\text { G } \\
\text { G } \\
\text { G } \\
\text { G } \\
\text { G } \\
P \\
\text { M } \\
\text { M } \\
\mathrm{P} \\
\mathrm{M} \\
\mathrm{M} \\
\mathrm{P} \\
\mathrm{G} \\
\mathrm{G} \\
\mathrm{G}\end{array}$ & $\begin{array}{l}\text { 1R-1, } 52-53 \mathrm{~cm} \\
1 \mathrm{R}-1,120-121 \mathrm{~cm} \\
1 \mathrm{R}-2,85-86 \mathrm{~cm} \\
1 \mathrm{R}-3,81-82 \mathrm{~cm} \\
1 \mathrm{R}-4,17-18 \mathrm{~cm} \\
1 \mathrm{R}-6,39-40 \mathrm{~cm} \\
1 \mathrm{R}, \mathrm{CC} \\
2 \mathrm{R}-1,25-26 \mathrm{~cm} \\
2 \mathrm{R}-1,118-119 \mathrm{~cm} \\
3 \mathrm{R}-2,138 \mathrm{~cm} \\
3 \mathrm{R}-3,110 \mathrm{~cm} \\
6 \mathrm{R}-2,67 \mathrm{~cm} \\
6 \mathrm{R}-3,43 \mathrm{~cm} \\
6 \mathrm{R}-3,51 \mathrm{~cm} \\
6 \mathrm{R}-3,55 \mathrm{~cm}\end{array}$ & $\begin{array}{l}\mathrm{F} \\
\mathrm{R} \\
\mathrm{F} \\
\mathrm{C} \\
. \\
\dot{\mathrm{R}} \\
\mathrm{R} \\
\mathrm{R} \\
\mathrm{F}\end{array}$ & $\begin{array}{l}\mathrm{F} \\
\mathrm{F} \\
\mathrm{F} \\
\mathrm{R} \\
\mathrm{C} \\
\mathrm{R} \\
\mathrm{R} \\
\mathrm{F} \\
\mathrm{R} \\
\mathrm{F} \\
\mathrm{F} \\
\dot{C} \\
\mathrm{C} \\
\mathrm{F}\end{array}$ & $\begin{array}{l}: \\
: \\
: \\
: \\
: \\
: \\
: \\
:\end{array}$ & & $\therefore:$ & 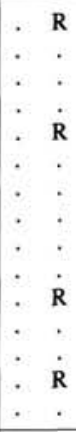 & 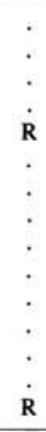 & $\begin{array}{ll}: & \mathbf{F} \\
: & \mathrm{F} \\
: & \mathrm{R} \\
: & \mathrm{R} \\
: & \mathrm{R} \\
: & \mathrm{R} \\
: & \mathrm{F} \\
: & \mathrm{F} \\
: & \mathrm{C} \\
: & \mathrm{C} \\
\end{array}$ & $\begin{array}{l}\text { A } \\
\text { A } \\
\text { C } \\
\text { C } \\
\text { A } \\
\text { F } \\
\text { F } \\
\text { C } \\
\text { C } \\
\text { A } \\
\text { A } \\
\text { F } \\
\text { A } \\
\text { C } \\
\text { A }\end{array}$ & $\begin{array}{l}\mathrm{F} \\
\mathrm{F} \\
\mathrm{R} \\
\mathrm{R} \\
\mathrm{R} \\
\mathrm{R} \\
\dot{\mathrm{F}} \\
\mathrm{F} \\
\mathrm{F} \\
\mathrm{F} \\
\mathrm{R} \\
\mathrm{F} \\
\mathrm{F} \\
\mathrm{F}\end{array}$ & 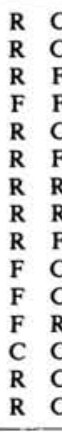 & 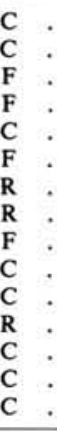 & & $\begin{array}{l}\mathrm{F} \\
\mathrm{F} \\
\mathrm{R} \\
\dot{\mathrm{F}} \\
\dot{\mathrm{F}} \\
\mathrm{F} \\
\dot{\mathrm{F}} \\
\dot{\mathrm{F}} \\
\mathrm{R} \\
\dot{\mathrm{C}} \\
\mathrm{R} \\
\mathrm{F}\end{array}$ & 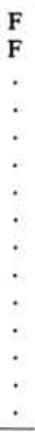 \\
\hline $\begin{array}{c}\text { early } \\
\text { Albian } \\
\text { to } \\
\text { late } \\
\text { Aptian }\end{array}$ & $\begin{array}{c}\text { Rhagodiscus } \\
\text { angustus } \\
\text { Subzone } \\
\text { CC7b }\end{array}$ & IV & $\begin{array}{l}\text { B } \\
\text { C } \\
\text { A } \\
\text { A } \\
\text { A } \\
\text { VA } \\
\text { VA } \\
\text { VA } \\
\text { A } \\
\text { A } \\
\text { C } \\
\text { A }\end{array}$ & $\begin{array}{l}M \\
\text { G } \\
\text { G } \\
\text { G } \\
\text { G } \\
\text { G } \\
\text { G } \\
\text { G } \\
\text { G } \\
\text { M } \\
\text { M }\end{array}$ & $\begin{array}{l}6 \mathrm{R}-3,77 \mathrm{~cm} \\
6 \mathrm{R}-3,78 \mathrm{~cm} \\
6 \mathrm{R}-3,91 \mathrm{~cm} \\
6 \mathrm{R}-3,123 \mathrm{~cm} \\
6 \mathrm{R}-4,8-9 \mathrm{~cm} \\
6 \mathrm{R}, \mathrm{CC} \\
7 \mathrm{R}-1,115-116 \mathrm{~cm} \\
7 \mathrm{R}-2,114-115 \mathrm{~cm} \\
8 \mathrm{R}-1,59-60 \mathrm{~cm} \\
8 \mathrm{R}-2,13-14 \mathrm{~cm} \\
8 \mathrm{R}-3,90-91 \mathrm{~cm} \\
8 \mathrm{R}-4,76-77 \mathrm{~cm}\end{array}$ & $\begin{array}{l}\mathrm{C} \\
\mathrm{C} \\
\mathrm{C} \\
\mathrm{F} \\
\mathrm{C} \\
\mathrm{C} \\
\mathrm{C} \\
\mathrm{C}\end{array}$ & $\begin{array}{l}\text { C } \\
\text { F } \\
\text { C } \\
\text { F } \\
\text { F } \\
\text { F } \\
\text { C } \\
\text { C } \\
\text { C } \\
\text { F } \\
\text { F }\end{array}$ & $\begin{array}{l}\dot{\mathrm{F}} \\
\dot{\mathrm{F}} \\
\dot{.} \\
\dot{.}\end{array}$ & $\begin{array}{l}\dot{.} \\
\dot{\mathrm{C}} \\
\dot{.} \\
\dot{.} \\
\dot{\mathrm{R}}\end{array}$ & $\begin{array}{l}\text { : } \\
\text { : } \\
\text {. } \\
\text {. }\end{array}$ & $\begin{array}{l}: \\
: \\
:\end{array}:$ & $\begin{array}{l}\dot{ } \\
\dot{\mathrm{R}} \\
\mathrm{R} \\
\mathrm{R} \\
\dot{.} \\
\dot{\mathrm{R}} \\
\dot{\mathrm{R}} \\
\dot{.}\end{array}$ & 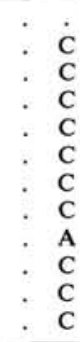 & $\begin{array}{l}\text { A } \\
\text { C } \\
\text { A } \\
\text { C } \\
\text { A } \\
\text { C } \\
\text { A } \\
\text { A } \\
\text { C } \\
\text { C } \\
\text { C }\end{array}$ & $\begin{array}{l}\dot{\mathrm{F}} \\
\mathrm{F} \\
\mathrm{F} \\
\mathrm{R} \\
\mathrm{F} \\
\mathrm{F} \\
\mathrm{F} \\
\mathrm{F} \\
\mathrm{R} \\
\mathrm{F} \\
\mathrm{R}\end{array}$ & $\begin{array}{ll}\dot{R} & \text { C } \\
\mathrm{F} & \mathrm{C} \\
\dot{\mathrm{R}} & \mathrm{C} \\
\mathrm{C} & \mathrm{F} \\
\dot{\mathrm{R}} & \mathrm{C} \\
\mathrm{F} & \mathrm{C} \\
\mathrm{C} & \mathrm{C} \\
\mathrm{F} & \mathrm{C} \\
\mathrm{R} & \mathrm{C} \\
. & \mathrm{C}\end{array}$ & $\begin{array}{l}\text { C } \\
\text { C } \\
\text { C } \\
\text { C } \\
\text { F } \\
C \\
C \\
C \\
C \\
C \\
C \\
C \\
C\end{array}$ & & $\begin{array}{l}\dot{C} \\
\text { F } \\
\text { C } \\
\text { F } \\
\text { F } \\
\text { C } \\
\text { C } \\
\text { C } \\
\text { F } \\
\text { R } \\
\text { F }\end{array}$ & $\begin{array}{l}\dot{\mathrm{R}} \\
\dot{\mathrm{R}} \\
\dot{\mathrm{R}} \\
\dot{\mathrm{R}} \\
\dot{\vec{R}} \\
\dot{.}\end{array}$ \\
\hline $\begin{array}{c}\text { early } \\
\text { Aptian }\end{array}$ & $\begin{array}{c}\text { Hayesites } \\
\text { irregularis } \\
\text { Subzone } \\
\text { CC7a }\end{array}$ & V & $\begin{array}{l}\text { C } \\
\text { VA } \\
\text { VA } \\
\text { A } \\
\text { A } \\
\text { A } \\
\text { A } \\
\text { A } \\
\text { A } \\
\text { A } \\
\text { A } \\
\text { A } \\
\text { A } \\
\text { A }\end{array}$ & $\begin{array}{l}\text { M } \\
G \\
G \\
M \\
M \\
M \\
G \\
M \\
P \\
G \\
G \\
G \\
M \\
P\end{array}$ & $\begin{array}{l}8 \mathrm{R}-4,121-122 \mathrm{~cm} \\
9 \mathrm{R}-1,57-58 \mathrm{~cm} \\
9 \mathrm{R}-3,139 \mathrm{~cm} \\
9 \mathrm{R}-4,37-38 \mathrm{~cm} \\
9 \mathrm{R}, \mathrm{CC} \\
10 \mathrm{R}-2,56 \mathrm{~cm} \\
10 \mathrm{R}-3,133 \mathrm{~cm} \\
10 \mathrm{R}-4,15-16 \mathrm{~cm} \\
10 \mathrm{R}-4,46 \mathrm{~cm} \\
10 \mathrm{R}, \mathrm{CC} \\
11 \mathrm{R}-2,55-56 \mathrm{~cm} \\
11 \mathrm{R}-2,60 \mathrm{~cm} \\
11 \mathrm{R}-2,108 \mathrm{~cm} \\
11 \mathrm{R}-3,76 \mathrm{~cm} \\
\end{array}$ & $\begin{array}{l}\mathrm{C} \\
\mathrm{C} \\
\mathrm{C} \\
\mathrm{C}\end{array}$ & $\begin{array}{l}\mathrm{R} \\
\dot{0} \\
\dot{C} \\
\mathrm{C} \\
\mathrm{C} \\
\mathrm{F} \\
\mathrm{F} \\
\mathrm{F} \\
\mathrm{F} \\
\mathrm{F} \\
\mathrm{F} \\
\mathrm{F} \\
\mathrm{R}\end{array}$ & $\begin{array}{l}\dot{ } \\
\dot{\mathrm{F}} \\
\mathrm{F} \\
\mathrm{F} \\
\mathrm{F} \\
\dot{\mathrm{F}} \\
\dot{\mathrm{R}} \\
\mathrm{R} \\
\mathrm{F} \\
\mathrm{R}\end{array}$ & $\begin{array}{l}\dot{.} \\
\dot{\mathrm{R}} \\
\dot{5} \\
\dot{.} \\
\dot{.} \\
\dot{.}\end{array}$ & $\begin{array}{l}\text {. } \\
\text { R } \\
\mathrm{R} \\
\mathrm{R} \\
. \\
\dot{ } \\
\dot{ }\end{array}$ & $\begin{array}{ll}\cdot & : \\
: & : \\
: & : \\
: & \dot{\mathrm{R}} \\
: & : \\
: & : \\
: & \mathrm{R} \\
. & \dot{\mathrm{F}} \\
. & .\end{array}$ & $\begin{array}{l}\dot{ } \\
\dot{0} \\
\dot{\mathrm{R}} \\
\mathrm{R} \\
\dot{\mathrm{R}} \\
\dot{\mathrm{R}} \\
\dot{\mathrm{R}}\end{array}$ & $\begin{array}{ll}. & \mathrm{C} \\
: & \mathrm{F} \\
: & \mathrm{C} \\
: & \mathrm{C} \\
: & \mathrm{C} \\
: & \text { C } \\
: & \mathrm{C} \\
: & \mathrm{C} \\
: & \mathrm{C} \\
: & \mathrm{C} \\
: & \mathrm{C} \\
: & \mathrm{C} \\
. & .\end{array}$ & $\begin{array}{l}\text { A } \\
\text { C } \\
\text { V } \\
\text { A } \\
\text { A } \\
\text { A } \\
\text { A } \\
\text { A } \\
\text { A } \\
\text { A } \\
\text { A } \\
\text { A } \\
\text { A }\end{array}$ & $\begin{array}{l}\mathrm{F} \\
\mathrm{R} \\
\mathrm{F} \\
\mathrm{F} \\
\mathrm{C} \\
\mathrm{F} \\
\mathrm{F} \\
\dot{\mathrm{F}} \\
\mathrm{F} \\
\dot{\mathrm{R}} \\
\dot{\mathrm{F}}\end{array}$ &  & $\begin{array}{ll}\mathrm{C} & \text { : } \\
\mathrm{C} & \text { : } \\
\mathrm{F} & \text { : } \\
\mathrm{C} & \text { : } \\
\mathrm{C} & \text { : } \\
\mathrm{C} & \text { : } \\
\mathrm{C} & \text { : } \\
\mathrm{R} & \text { : } \\
\mathrm{R} & \text { : } \\
\mathrm{R} & \text { : } \\
\mathrm{C} & \text { : } \\
\mathrm{C} & \text { : } \\
\mathrm{C} & \text { : } \\
\mathrm{C} & \text { : }\end{array}$ & & $\begin{array}{l}\text { C } \\
\text { F } \\
\text { C } \\
\text { F } \\
\text { C } \\
\text { C } \\
\text { C } \\
\text { F } \\
\text { C } \\
\text { F } \\
\text { C } \\
\text { C } \\
\text { C }\end{array}$ & $\begin{array}{l} \\
\vdots \\
\vdots \\
\dot{\mathrm{F}} \\
\mathrm{F} \\
\mathrm{C} \\
\end{array}$ \\
\hline $\begin{array}{c}\text { late } \\
\text { Barremian }\end{array}$ & $\begin{array}{c}\text { Micrantho- } \\
\text { lithus } \\
\text { hoschulzii } \\
\text { Zone } \\
\text { CC6 }\end{array}$ & VI & $\begin{array}{l}\text { A } \\
\text { A } \\
\text { VA } \\
\text { A } \\
\text { A } \\
\text { A } \\
\text { A } \\
\text { C } \\
\text { A } \\
\text { C } \\
\text { VA } \\
\text { A } \\
\text { A } \\
\text { A } \\
\text { A } \\
\text { A } \\
\text { A } \\
\text { A } \\
\text { A } \\
\text { A }\end{array}$ & $\begin{array}{l}M \\
G \\
G \\
G \\
G \\
G \\
G \\
M \\
G \\
P \\
\text { G } \\
M \\
M \\
M \\
M \\
M \\
G \\
G \\
M \\
P \\
M\end{array}$ & $\begin{array}{l}12 \mathrm{R}-1,27-28 \mathrm{~cm} \\
12 \mathrm{R}-2,44 \mathrm{~cm} \\
12 \mathrm{R}-3,103 \mathrm{~cm} \\
12 \mathrm{R}-3,107 \mathrm{~cm} \\
12 \mathrm{R}-4,91 \mathrm{~cm} \\
12 \mathrm{R}-5,96 \mathrm{~cm} \\
12 \mathrm{R}-6,4-5 \mathrm{~cm} \\
13 \mathrm{R}-1,45-46 \mathrm{~cm} \\
13 \mathrm{R}-2,36 \mathrm{~cm} \\
13 \mathrm{R}-4,11 \mathrm{~cm} \\
13 \mathrm{R}-5,27 \mathrm{~cm} \\
14 \mathrm{R}-3,46 \mathrm{~cm} \\
14 \mathrm{R}-4,31 \mathrm{~cm} \\
15 \mathrm{R}-2,40 \mathrm{~cm} \\
15 \mathrm{R}-3,27 \mathrm{~cm} \\
15 \mathrm{R}-5,40 \mathrm{~cm} \\
16 \mathrm{R}-2,35 \mathrm{~cm} \\
16 \mathrm{R}-4,61 \mathrm{~cm} \\
16 \mathrm{R}-5,36 \mathrm{~cm} \\
16 \mathrm{R}-8,28 \mathrm{~cm}\end{array}$ & $\begin{array}{l}\mathrm{F} \\
\mathrm{F} \\
\mathrm{C} \\
\mathrm{C} \\
\mathrm{C} \\
\mathrm{F}\end{array}$ & $\begin{array}{l}\mathrm{R} \\
\mathrm{R} \\
\mathrm{F} \\
\mathrm{F} \\
\mathrm{F} \\
\mathrm{F} \\
\mathrm{F} \\
\dot{\mathrm{F}} \\
\dot{\mathrm{R}} \\
\dot{\mathrm{R}} \\
\dot{:} \\
\vdots \\
\vdots\end{array}$ & $\begin{array}{l}\mathrm{R} \\
\dot{\mathrm{R}} \\
\mathrm{F} \\
\mathrm{R} \\
\mathrm{F} \\
\mathrm{R} \\
\dot{\mathrm{R}} \\
\dot{\mathrm{R}} \\
\\
\\
\\
\\
\\
\mathrm{R} \\
\mathrm{R}\end{array}$ & $\begin{array}{l} \\
\dot{\mathrm{R}} \\
\mathrm{F} \\
\mathrm{F} \\
\mathrm{R} \\
\vdots \\
\vdots\end{array}$ & $\vdots$ & $\begin{array}{ll}: & \vdots \\
\vdots & \vdots \\
\vdots & \text { R } \\
\vdots & \vdots \\
\vdots & \vdots \\
\vdots & \vdots \\
\vdots & \vdots \\
\vdots & \vdots \\
\mathrm{R} & \vdots \\
\mathrm{R} & \vdots \\
: & \vdots\end{array}$ & $\begin{array}{l}\dot{ } \\
\vdots \\
\dot{\mathrm{R}} \\
\dot{\mathrm{R}} \\
\dot{\mathrm{R}} \\
\dot{ } \\
\dot{\mathrm{C}}\end{array}$ & 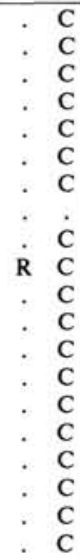 & $\begin{array}{l}\text { A } \\
\text { A } \\
\text { C } \\
\text { A } \\
\text { A } \\
\text { A } \\
\text { A } \\
\text { A } \\
\text { C } \\
\text { A } \\
\text { A } \\
\text { A } \\
\text { A } \\
\text { A } \\
\text { A } \\
\text { A } \\
\text { A } \\
\text { A } \\
\text { A }\end{array}$ & $\begin{array}{l}\mathrm{R} \\
\mathrm{R} \\
\dot{\mathrm{F}} \\
\mathrm{F} \\
\mathrm{F} \\
\mathrm{F} \\
\dot{\mathrm{F}} \\
\dot{\cdot} \\
\dot{\mathrm{R}} \\
\mathrm{R} \\
\mathrm{R} \\
\dot{\mathrm{R}} \\
\mathrm{R} \\
\mathrm{R} \\
\mathrm{F} \\
\mathrm{F}\end{array}$ & $\begin{array}{ll}\mathrm{F} & \mathrm{C} \\
\mathrm{R} & \mathrm{F} \\
\dot{2} & \mathrm{C} \\
\mathrm{R} & \mathrm{C} \\
\mathrm{R} & \mathrm{C} \\
\mathrm{F} & \mathrm{C} \\
\mathrm{F} & \mathrm{C} \\
\dot{R} & \mathrm{C} \\
\mathrm{R} & \mathrm{F} \\
\mathrm{R} & \mathrm{F} \\
\mathrm{R} & \mathrm{F} \\
. & \mathrm{R} \\
: & \mathrm{C} \\
: & \mathrm{C} \\
\dot{2} & \mathrm{C} \\
\mathrm{R} & \mathrm{C} \\
\mathrm{F} & \mathrm{C} \\
\mathrm{R} & \mathrm{C} \\
\mathrm{R} & \mathrm{C} \\
\mathrm{R} & \mathrm{C}\end{array}$ & 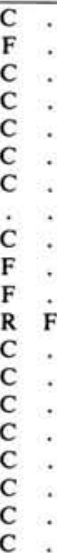 & & $\begin{array}{l}\mathrm{C} \\
\mathrm{F} \\
\mathrm{F} \\
\mathrm{F} \\
\mathrm{C} \\
\mathrm{C} \\
\mathrm{C} \\
\dot{C} \\
\mathrm{~F} \\
\mathrm{~F} \\
\mathrm{C} \\
\mathrm{F} \\
\mathrm{C} \\
\mathrm{C} \\
\mathrm{F} \\
\mathrm{C} \\
\mathrm{F} \\
\mathrm{R} \\
\mathrm{F}\end{array}$ & $\begin{array}{l}\dot{ } \\
\dot{\mathrm{F}} \\
\mathrm{C} \\
\mathrm{C} \\
\mathrm{C} \\
\mathrm{C} \\
\dot{\mathrm{F}} \\
\dot{ }\end{array}$ \\
\hline
\end{tabular}




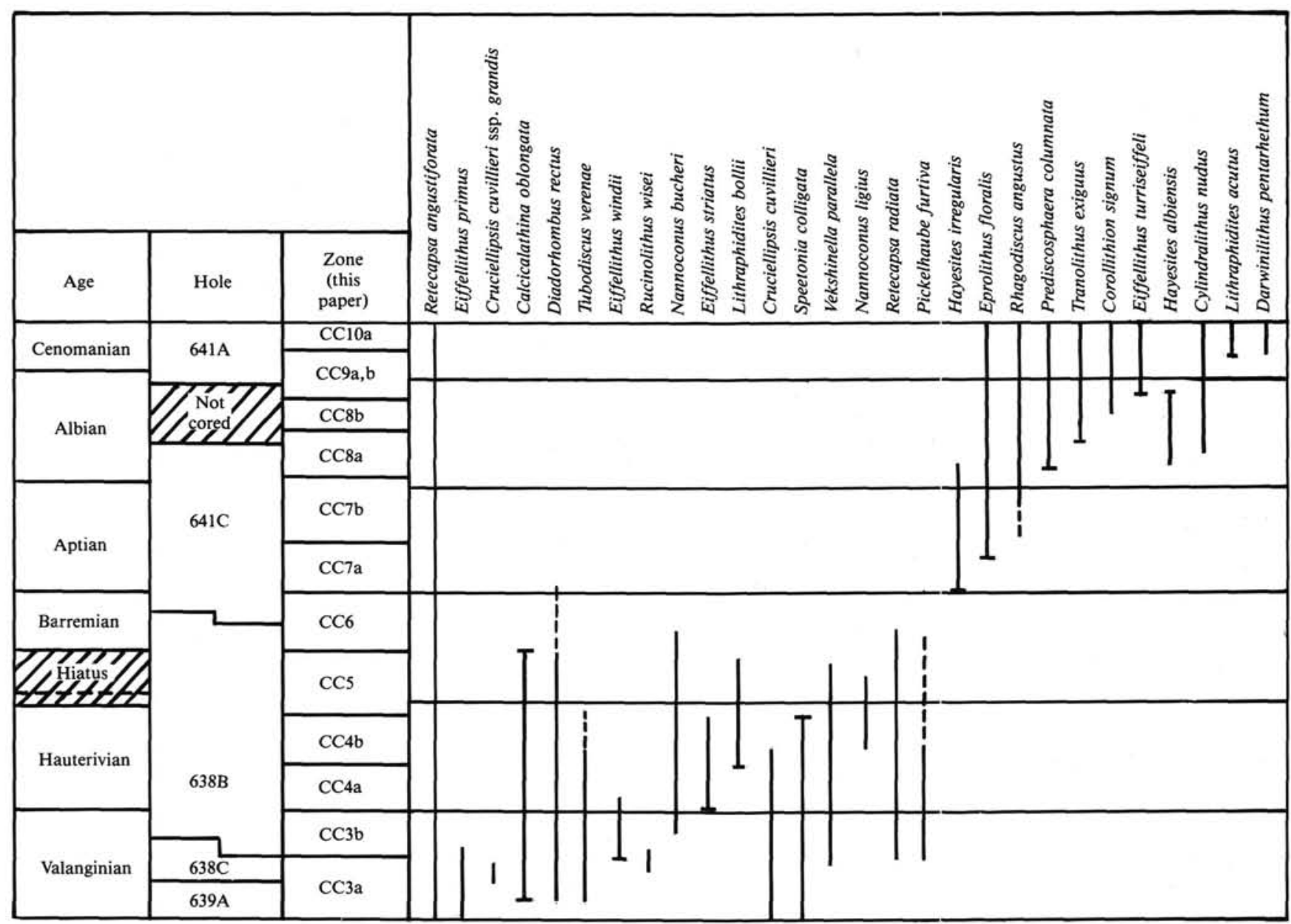

Figure 5. Ranges of index nannofossils and other important species considered.

Radiolithus planus appears near the middle of $\mathrm{CC} 7 \mathrm{~b}$, whereas $H$. albiensis and $R$. angustus appear near the very top of CC7b. Percivalia fenestrata disappears near the top of this interval.

The first occurrence of $P$. columnata, defining the base of $\mathrm{CC} 8$, occurs at the contact between the clastic carbonate turbidites and dark green and black claystones. This change in sedimentation style and correlation to the seismic stratigraphy suggests an unconformity. Species that have their first occurrence in the lower Albian of Site 641 include $P$. columnata, Prediscosphaera spinosa, and Cylindralithus nudus. The earliest cylindraliths previously reported were from the uppermost Albianlower Cenomanian of Leg 77 (Watkins and Bowdler, 1984). C. nudus is most likely the earliest representative of the genus. $A x$ opodorhabdus albianus, a species Thierstein (1976) uses as a zonal marker in the mid-Albian, is present in the uppermost sample of Hole $641 \mathrm{C}$. The first occurrences of $T$. phacelosus, Cribrosphaerella ehrenbergi, and Corollithion signum, which are used to divide the lower and upper Albian (CC8a/CC8b), were not observed because $50 \mathrm{~m}$ of the lower to middle Albian section in Hole $641 \mathrm{C}$ was washed in response to time constraints. The lowermost samples in Hole $641 \mathrm{~A}$ contain the taxa T. exiguus, Cruciribrum striatum striatum, C. ehrenbergi, E. turriseiffeli, Lithraphidites alatus, and Prediscosphaera cretacea. These species were not present in the upper part of Hole $641 \mathrm{C}$; therefore, their first occurrences must be within the $50-\mathrm{m}$ washed interval in the hole.

The Albian/Cenomanian boundary cannot be accurately resolved using nannofossils. Thierstein (1976) used the first occurrence of $L$. alatus to define this boundary; however, both Ver- beek (1977) and Watkins and Bowdler (1984) extended its range into the upper Albian. The Albian/Cenomanian boundary is placed within Core 103-641A-7X using foraminifer biostratigraphy. The interval from Sample 103-641A-7X-1, 3-4 cm, to Section 103-641A-7X, CC, is placed in the $P$. spinosa Subzone (CC9b) of late Albian to early Cenomanian age. The first occurrences of Lithraphidites acutus ssp. eccentricus, Lithraphidites acutus ssp. acutus, and Darwinilithus pentarhethum are in Sample 103-641A-6X, CC $(40 \mathrm{~cm})$. Only the core catcher of Core 103-641A-6X contained nannofossils (below a black barren zeolitic claystone), which indicate an early to mid-Cenomanian age, and this short interval is assigned to the $L$. acutus Zone (CC10). Watkins and Bowdler (1984) described L. acutus spp. eccentricus and $D$. petarhethum from the Cenomanian recovered on DSDP Leg 77. He explained that L. acutus ssp. acutus, a marker for the middle Cenomanian, was not present and that L. acutus spp. eccentricus was probably an "ecologic variant" of L. acutus spp. acutus. The co-occurrence of these subspecies in samples from Section 103-641A-6X, CC, confirms that they are indeed subspecific variants.

\section{SUMMARY, CONCLUSIONS, AND DEPOSITIONAL HISTORY}

The composite sequence for the Lower to middle Cretaceous of ODP Leg 103 is nearly continuous from the lower Valanginian to middle Cenomanian. This makes it an excellent section for the study and documentation of calcareous nannofossils. Most of the highly varied lithologies within the column contain 
Table 5. Relative abundance of nannofossils, Hole 641A.

\begin{tabular}{|c|c|c|c|c|c|c|c|c|c|c|c|c|c|c|c|c|c|c|c|c|c|c|c|c|c|c|c|c|}
\hline Age & $\begin{array}{l}\text { Sissingh } \\
\text { zonation } \\
(1977)\end{array}$ & $\begin{array}{l}\text { Lithologic } \\
\text { unit }\end{array}$ & 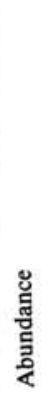 & 总 & Sample & 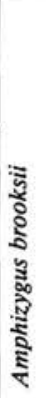 & 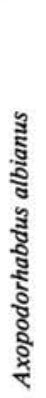 & 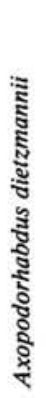 & 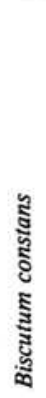 & 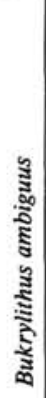 & 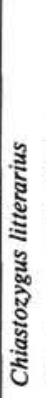 & 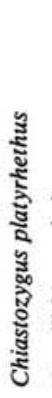 & 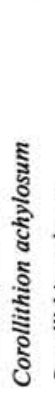 &  & 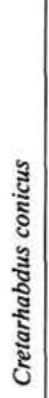 &  & 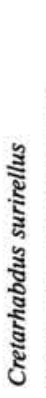 & 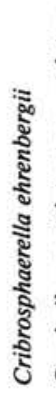 & 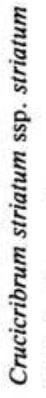 & 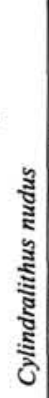 & 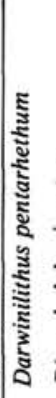 & 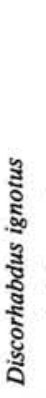 & 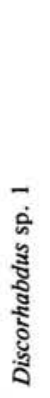 & 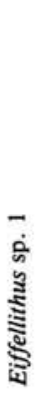 & 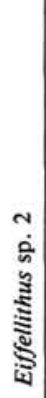 & m & 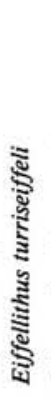 &  \\
\hline $\begin{array}{c}\text { early } \\
\text { to } \\
\text { middle } \\
\text { Cenomanian }\end{array}$ & $\mathrm{CC} 10 \mathrm{a}$ & & $\begin{array}{l}\text { C } \\
\text { VA } \\
\text { VA } \\
\text { A }\end{array}$ & $\begin{array}{l}P \\
G \\
G \\
G\end{array}$ & $\begin{array}{l}6 \mathrm{X}, \mathrm{CC}(21-24 \mathrm{~cm}) \\
6 \mathrm{X}, \mathrm{CC}(27 \mathrm{~cm}) \\
6 \mathrm{X}, \mathrm{CC}(29 \mathrm{~cm}) \\
6 \mathrm{X}, \mathrm{CC}(40 \mathrm{~cm})\end{array}$ & $\dot{\mathrm{C}}$ & $\begin{array}{l}\dot{R} \\
\mathrm{~F} \\
\mathrm{R}\end{array}$ & $\dot{\dot{R}}$ & $\begin{array}{l}\dot{A} \\
\mathrm{~A} \\
\mathrm{~A}\end{array}$ & $\dot{.}$ & $\dot{\vec{F}}$ & $\begin{array}{l}\dot{\mathrm{F}} \\
\mathrm{F} \\
\mathrm{R}\end{array}$ & $\dot{\dot{.}}$ & $\begin{array}{l}\dot{R} \\
\mathrm{R}\end{array}$ & $\begin{array}{l}\dot{\mathrm{F}} \\
\mathrm{R}\end{array}$ & $\dot{i}$ & $\begin{array}{l}\dot{C} \\
\text { C } \\
\text { F }\end{array}$ & $\begin{array}{l}\dot{\mathrm{F}} \\
\mathrm{R}\end{array}$ & $\begin{array}{l}\dot{\mathrm{F}} \\
\mathrm{C} \\
\mathrm{C}\end{array}$ & $\begin{array}{c}\mathrm{R} \\
\dot{\mathrm{R}}\end{array}$ & $\begin{array}{l}\mathrm{R} \\
\mathrm{F} \\
\dot{\mathrm{R}}\end{array}$ & $\begin{array}{l}\dot{\mathrm{C}} \\
\dot{\mathrm{F}}\end{array}$ & $\dot{\dot{c}}$ & $\dot{\mathrm{F}}$ & $\begin{array}{c}\dot{\mathrm{F}} \\
\dot{\cdot}\end{array}$ & $\begin{array}{l}\dot{\mathrm{R}} \\
\dot{\cdot}\end{array}$ & $\begin{array}{l}\mathrm{R} \\
\mathrm{C} \\
\mathrm{C} \\
\mathrm{C}\end{array}$ & \\
\hline $\begin{array}{c}\text { early } \\
\text { Cenomanian } \\
\text { to } \\
\text { late } \\
\text { Albian }\end{array}$ & $\mathrm{CC} 9 \mathrm{~b}$ & III & $\begin{array}{c}\text { A } \\
\text { A } \\
\text { A } \\
\text { A } \\
\text { A } \\
\text { VA } \\
\text { C } \\
\text { A } \\
\text { A }\end{array}$ & $\begin{array}{l}\text { G } \\
G \\
M \\
G \\
M \\
G \\
P \\
G \\
G\end{array}$ & $\begin{array}{l}7 X-1,3-4 \mathrm{~cm} \\
7 X-1,20-21 \mathrm{~cm} \\
7 X-1,91-92 \mathrm{~cm} \\
7 X-3,48-49 \mathrm{~cm} \\
7 X-4,50-51 \mathrm{~cm} \\
7 X-5,30-31 \mathrm{~cm} \\
7 X-5,102-103 \mathrm{~cm} \\
7 X-6,14-15 \mathrm{~cm} \\
7 X, C C\end{array}$ & $\begin{array}{l}\text { C } \\
\text { F } \\
F \\
F \\
\text { R } \\
\text { C } \\
F \\
F \\
\text { C }\end{array}$ & $\begin{array}{l}\mathrm{F} \\
\mathrm{R} \\
\mathrm{F} \\
\mathrm{F} \\
\mathrm{R} \\
\mathrm{C} \\
\mathrm{R} \\
\mathrm{F} \\
\mathrm{F}\end{array}$ & $\begin{array}{l}\mathrm{R} \\
\mathrm{R} \\
\mathrm{R} \\
\dot{\cdot} \\
\dot{\cdot} \\
\dot{\cdot} \\
\dot{.} \\
\dot{.}\end{array}$ & $\begin{array}{l}\text { A } \\
\text { A } \\
\text { A } \\
\text { A } \\
\text { A } \\
\text { A } \\
\text { C } \\
\text { A } \\
\text { A }\end{array}$ & $\begin{array}{c}\mathbf{R} \\
\dot{\mathrm{R}} \\
\mathrm{R} \\
\dot{\cdot} \\
\dot{\cdot} \\
\dot{\cdot}\end{array}$ & $\begin{array}{l}\mathrm{R} \\
\mathrm{R} \\
\mathrm{F} \\
\mathrm{F} \\
\mathrm{R} \\
\mathrm{R} \\
\mathrm{R} \\
\dot{\mathrm{R}}\end{array}$ & $\begin{array}{c}\mathbf{R} \\
\mathbf{R} \\
\mathbf{R} \\
\dot{\cdot} \\
\dot{\cdot} \\
\dot{\cdot} \\
\dot{\mathbf{R}}\end{array}$ & $\begin{array}{l}\dot{\mathrm{R}} \\
\dot{\cdot} \\
\dot{\cdot} \\
\dot{.}\end{array}$ & $\begin{array}{l}\dot{\mathrm{R}} \\
\dot{\cdot} \\
\dot{\cdot} \\
\dot{\cdot}\end{array}$ & $\begin{array}{c}\dot{\mathrm{F}} \\
\mathrm{R} \\
\dot{\mathrm{F}} \\
\dot{\mathrm{F}} \\
\dot{\mathrm{R}}\end{array}$ & $\begin{array}{c}\mathrm{R} \\
\mathrm{R} \\
\mathrm{R} \\
\mathrm{R} \\
\mathrm{R} \\
\dot{\cdot} \\
\dot{\mathrm{R}}\end{array}$ & $\begin{array}{l}\mathrm{F} \\
\dot{\mathrm{R}} \\
\mathrm{F} \\
\mathrm{F} \\
\mathrm{R} \\
\mathrm{F} \\
\mathrm{C} \\
\mathrm{C}\end{array}$ & $\begin{array}{c}\mathrm{R} \\
\dot{\mathrm{F}} \\
\mathrm{F} \\
\dot{\mathrm{F}} \\
\dot{\mathrm{R}} \\
\dot{\mathrm{R}}\end{array}$ & $\begin{array}{l}\mathrm{C} \\
\mathrm{C} \\
\mathrm{C} \\
\mathrm{C} \\
\mathrm{F} \\
\mathrm{C} \\
\mathrm{R} \\
\mathrm{F} \\
\mathrm{F}\end{array}$ & $\begin{array}{c}\mathrm{R} \\
\dot{\mathrm{R}} \\
\mathrm{R} \\
\mathrm{R} \\
\dot{\mathrm{R}} \\
\mathrm{R} \\
\mathrm{F}\end{array}$ & 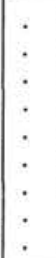 & $\begin{array}{l}\text { R } \\
\text { F } \\
\text { F } \\
\text { C } \\
\text { F } \\
\text { C } \\
\text { R } \\
\text { F } \\
\text { F }\end{array}$ & $\begin{array}{c}\mathrm{F} \\
\mathrm{R} \\
\mathrm{R} \\
\mathrm{R} \\
\dot{\mathrm{R}} \\
\dot{\mathrm{R}} \\
\mathrm{R}\end{array}$ & $\begin{array}{c}\dot{\mathrm{F}} \\
\mathrm{R} \\
\mathrm{R} \\
\mathrm{R} \\
\mathrm{R} \\
\dot{\mathrm{R}} \\
\mathrm{R}\end{array}$ & $\begin{array}{c}\dot{\mathrm{F}} \\
\mathrm{R} \\
\mathrm{R} \\
\mathrm{F} \\
\mathrm{R} \\
\mathrm{F} \\
\mathrm{R} \\
.\end{array}$ & $\begin{array}{l}\mathrm{R} \\
\mathrm{F} \\
\mathrm{R} \\
\mathrm{R} \\
\mathrm{R} \\
\mathrm{F} \\
\dot{\mathrm{R}} \\
\mathrm{R}\end{array}$ & $\begin{array}{l}\text { C } \\
C \\
C \\
C \\
C \\
C \\
F \\
C \\
C\end{array}$ & \\
\hline
\end{tabular}

$\mathrm{R}=$ Rare; $\mathrm{F}=$ Few $; \mathrm{C}=$ Common; $\mathrm{A}=$ Abundant; $\mathrm{VA}=$ Very abundant; ? = Questionably present; . = Not present.

nannofossils, with the exception of the lower Valanginian sandstones and a few cores of Albian black shale. Abundance and diversity vary greatly but are generally much higher than reported from most other Lower Cretaceous sequences. Preservation is highly variable within the different lithologies and is often variable within an individual sample. However, overall preservation throughout the column is moderate to good.

The nannofossil biostratigraphy reveals a nearly complete record of sedimentation from the lower Valanginian to middle $\mathrm{Ce}$ nomanian, although parts of the uppermost Hauterivian-lower Barremian are missing. The nannofossil assemblages evidence a complex evolution for the Galicia margin. The sequence of events is as follows:

1. In the early Valanginian, a 40-m-thick calpionellid-nannofossil marlstone was deposited on a shallow-water carbonate platform. Nannoconids dominate the assemblage.

2. During initial rifting and subsidence, terrigenous sands and clays were deposited by turbidites and interbedded with nannofossil marlstone of early to late Valanginian age. Shallowwater forms such as the nannoconids no longer dominate the assemblage; diversity and preservation increase considerably.

3. A siltstone-claystone-marlstone sequence deposited in fairly deep water during the late Valanginian and Hauterivian shows evidence of redeposition (creep and slump features; "Sedimentology" section; "Site 638" chapter; Boillot, Winterer, et al., 1987). Shallow-water forms become abundant as time passes, again dominating nannofossil assemblages; diversity of nannofossils is extremely high in the late early to late Hauterivian.

4. The latest stages of rifting occurred in the late Barremian and Aptian and resulted in deposition of a clastic calcareous turbidite sequence containing coarse shell lags, which include reworked, lower Barremian neritic nannofossils.

5. Further subsidence occurred as seafloor spreading began in the late Aptian-early Albian. Dark green to black carbonaceous claystones were deposited in deep water near or close to the CCD; nannofossil preservation, abundance, and diversity diminish.
6. In the latest Albian, a light green to gray nannofossil marlstone was deposited in deep water. The deposition of the nannofossil marlstone continued into the early to mid-Cenomanian.

7. Black carbonaceous claystone and brownish claystones devoid of calcareous nannofossils were deposited in deep water below the CCD in the Upper Cretaceous and possibly up into the Paleogene.

\section{ACKNOWLEDGMENTS}

We would like to thank Katharina Perch-Nielsen (Geological Institute, ETH, Zurich, Switzerland) and Timothy J. Bralower (Florida International University) for their critical and helpful review of the manuscript. The paper is based on a thesis submitted by the senior author to the Florida State University, Tallahassee, in partial fulfillment of the Master of Science degree. The senior author gives many thanks to Sherwood W. Wise for an early review of the manuscript and for his support and enthusiasum for this project and also thanks his wife, Ligia, for her support throughout this study. Taxonomic discussion with Sherwood Wise, Mitchener Covington (Texaco, New Orleans), and John Firth (FSU) were very helpful. Many thanks to James R. Breza (FSU) for computer and laboratory work.

\section{REFERENCES}

Black, M., 1971. Coccoliths of the Speeton Clay and Sutterby Marl. Proc. Yorksh. Geol. Soc., 38:381-424.

Blechschmidt, G., 1979. Biostratigraphy of calcareous nannofossils: Leg 47B, Deep Sea Drilling Project. In Sibuet, J.-C., Ryan, W.B.F., et al., Init. Repts, DSDP, 47, Pt. 2: Washington (U.S. Govt. Printing Office), 327-360.

Boillot, G., Winterer, E. L., et al., 1987. Proc. ODP, Init. Repts., 103: College Station, TX (Ocean Drilling Program).

Bralower, J. T., 1986. An integrated Mesozoic biochronology and magnetochronology [Pt. A, Ph.D dissert.]. Univ. of California, San Diego.

Covington, M., and Wise, S. W., 1987. Calcareous nannofossil biostratigraphy of a Lower Cretaceous deep sea fan complex: DSDP Leg 93 Site 603, lower continental rise off Cape Hatteras. In Van Hinte, J. E., Wise, S. W., Jr., et al., Init. Repts. DSDP, 93, Washington (U.S. Govt. Printing Office), 617-660. 
Table 5 (continued).

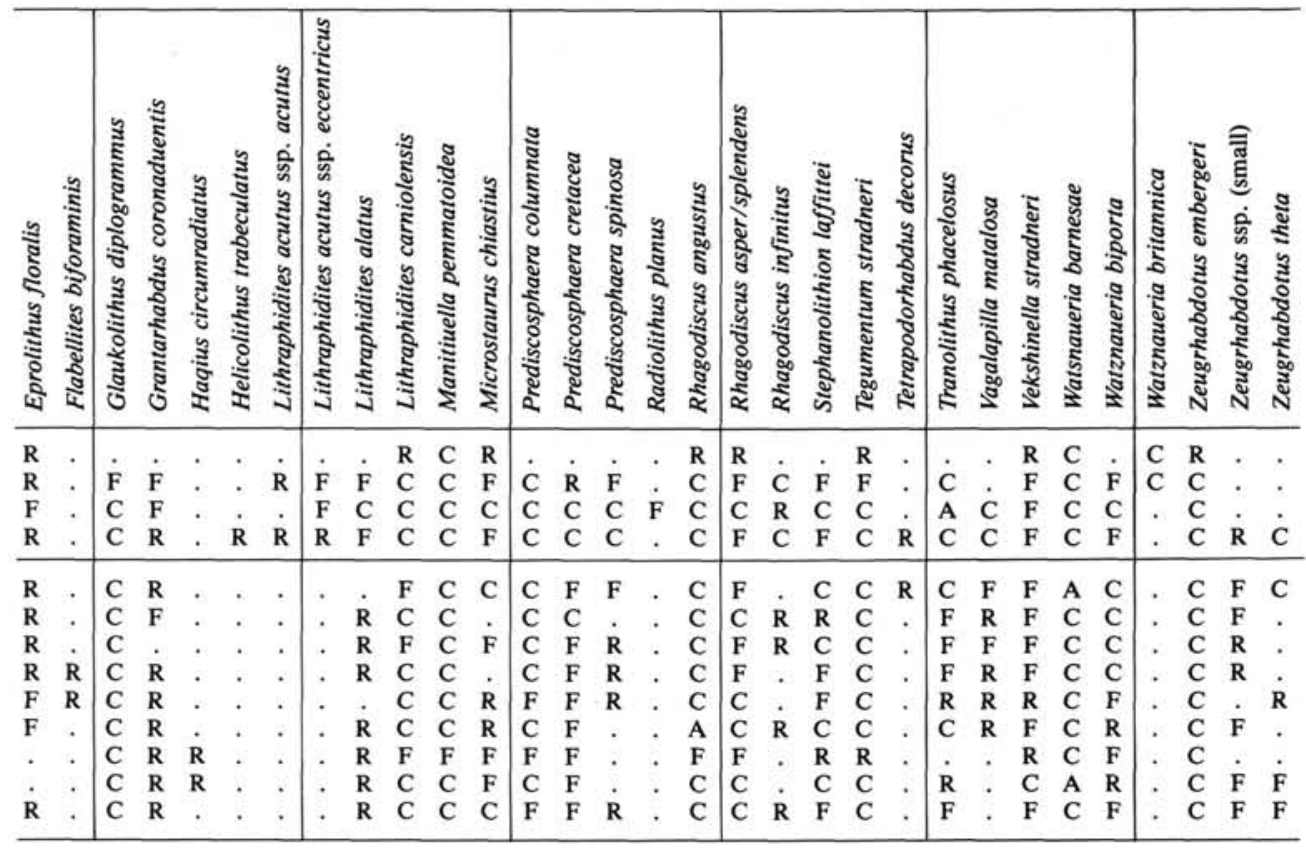

Gorka, H., 1957. Les Coccolithophorides du Maestrichtien superieur de Pologne. Acta Palaeontol. Polonica., 2:235-284.

Hay, W. W., 1970. Calcareous nannofossils from cores recovered on Leg 4. In Bader, R. G., Gerard, R. D., et al., Init. Repts. DSDP, 4: Washington (U.S. Govt. Printing Office), 455-501.

Jansa, L. F., Tucholke, B. E., Gradstein, F. M., and Sheridan, R. E., 1979. Mesozoic-Cenozoic sedimentary formations of the North American Basin, western North Atlantic. In Talwani, M., Hay, W. and Ryan, W.B.F. (Eds.), Deep Drilling Results in the Atlantic Ocean: Continental Margins and Paleoenvironment: Am. Geophys. Union, Maurice Ewing Ser., 3:1-57.

Kothe, V. A., 1981. Kalkiges Nannoplankton aus dem Unter-Hauterivian bis Unter-Barremium der Tongrube Moorberg/Sarstedt (UnterKriede, NW-Deutschland). Mitt. Geol. Inst. Univ. Hannover, 56: 110.

Loeblich, A. R., Jr., and Tappan, H., 1966. Annotated index and bibliography of the calcareous nannoplankton, I. Phycologia, 5:81-216. 1968. Annotated index and bibliography of the calcareous nannoplankton, II. J. Paleontol., 42:584-598.

1969. Annotated index and bibliography of the calcareous nannoplankton, III. J. Paleontol., 43:568-588.

1970a. Annotated index and bibliography of the calcareous nannoplankton, IV. J. Paleontol., 44:558-574.

1970b. Annotated index and bibliography of the calcareous nannoplankton, V. Phycologia, 9:157-174.

1971. Annotated index and bibliography of the calcareous nannoplankton, VI. Phycologia, 10:315-339.

1973. Annotated index and bibliography of the calcareous nannoplankton, VII. J. Paleontol., 47:715-759.

Manivit, H. 1971. Les nannofossiles calcaires du Crétacé français (de l'Aptien au Danien). Essai de biozonation appuyée sur les stratotypes [Thesis]. Univ. de Paris.

Manivit, H., Perch-Nielsen, K., Prins, B., and Verbeek, J. W., 1977. Mid Cretaceous calcareous nannofossil biostratigraphy. Proc. K. Ned. Akad. Wet. Ser. B: Palaeontol. Geol. Phys. Chem, 80:169-181.

Perch-Nielsen, K., 1979. Calcareous nannofossils from the Cretaceous between the North Sea and the Mediterranean. In Wiedmann, J. (Ed.), Aspekte der Kreide Europas: Int. Union Geol. Sci. Ser. A, 6: 223-272.

1984. Validation of new combinations. Int. Nannoplankton Assoc. Newsl., 6:42-46.
1985. Mesozoic calcareous nannofossils. In Bolli, H. M., Saunders, J. B., and Perch-Nielsen, K. (Eds.), Plankton Stratigraphy: Cambridge (Cambridge Univ. Press), 329-426.

Roth, P. H., 1973. Calcareous, Nannofossils-Leg 17, Deep Sea Drilling Project. In Winterer, E. L., Ewing, J. I., et al., Init. Repts. DSDP, 17: Washington (U.S. Govt. Printing Office), 695-795.

1978. Cretaceous nannoplankton biostratigraphy and oceanography of the northwestern Atlantic Ocean. In Benson, W. E., Sheridan, R. E., et al., Init. Repts. DSDP, 44: Washington (U.S. Govt. Printing Office), 731-759.

1983. Jurassic and Lower Cretaceous calcareous nannofossils in the western North Atlantic (Site 634): biostratigraphy, preservation, and some observations on biogeography and paleoceanography. In Sheridan, R. E., Gradstein, F. M., et al., Init. Repts. DSDP, 76: Washington (U.S. Govt. Printing Office), 587-621.

Roth, P. H., and Thierstein, H. R., 1972. Calcareous nannoplankton: Leg 14 of the Deep Sea Drilling Project. In Hayes, D. E., Pimm, A. C., et al., Init. Repts. DSDP, 14: Washington (U.S. Govt. Printing Office), 421-486.

Schlanger, S. O., and Douglas, R. G., 1974. The pelagic-ooze-chalklimestone transition and its implication for marine stratigraphy. Spec. Publ. Assoc. Sedimentol., 1:117-148.

Sibuet, J.-C., Ryan, W.B.F., et al., 1979. Init. Repts. DSDP, 47, Pt. 2: Washington (U.S. Govt. Printing Office).

Sissingh, W., 1977. Biostratigraphy of Cretaceous calcareous nannoplankton. Geol. Mijnbouw, 56:37-65.

Steinmetz, J. C., 1984. Bibliography and taxa of calcareous nannoplankton-III. Int. Nannoplankton Assoc. Newsl., 6:6-37. 1984b. Bibliography and taxa of calcareous nannoplanktonIV. Int. Nannoplankton Assoc. Newsl., 6:55-81. 1985. Bibliography and taxa of calcareous nannoplanktonV. Init. Nannoplankton Assoc. Newsl., 7:5-28.

1986. Bibliography and taxa of calcareous nannoplanktonVI. Init. Nannoplankton Assoc. Newsl., 8:66-87.

Thierstein, H. R., 1971. Tentative Lower Cretaceous calcareous nannoplankton zonation. Eclogae Geol. Helv., 64:459-488.

1973. Lower Cretaceous calcareous nannoplankton zonation. Abh. Geol. Bundesanst. (Austria), 29:1-52. 1976. Mesozoic calcareous nannoplankton biostratigraphy of marine sediments. Mar. Micropaleontol., 1:325-362. 
Van Heck, S. E., 1979a. Bibliography and taxa of calcareous nannoplankton. Int. Nannoplankton Assoc. Newsl., 1:AB1-AB5, A1-A2, B1-B27.

1979b. Bibliography and taxa of calcareous nannoplankton. Int. Nannoplankton Assoc. Newsl., 1:AB, VI, A13-A28, B28-B42. ,1980a. Bibliography and taxa of calcareous nannoplankton. Int. Nannoplankton Assoc. Newsl., 2:5-34.

$1980 \mathrm{~b}$. Bibliography and taxa of calcareous nannoplankton. Int. Nannoplankton Assoc. Newsl., 2:43-81.

1981a. Bibliography and taxa of calcareous nannoplankton. Int. Nannoplankton Assoc. Newsl., 3:4-41.

1981b. Bibliography and taxa of calcareous nannoplankton. Int. Nannoplankton Assoc. Newsl., 3:51-86.

1982a. Bibliography and taxa of calcareous nannoplankton. Int. Nannoplankton Assoc. Newsl., 4:7-50.

1982b. Bibliography and taxa of calcareous nannoplankton. Int. Nannoplankton Assoc. Newsl., 4:65-96.

1983. Bibliography and taxa of calcareous nannoplankton. Int. Nannoplankton Assoc. Newsl., 5:4-13.

Van Hinte, J. E., 1976. A Cretaceous time scale. Am. Assoc. Petrol. Geol. Bull., 60:498-516.

Verbeek, J. W., 1977. Calcareous nannoplankton biostratigraphy of middle and Upper Cretaceous deposits in Tunisia, southern Spain and France. Utrecht Micropaleontol. Bull., 16:1-157.

Watkins, D. K. and Bowdler, J. L., 1984. Cretaceous calcareous nannofossils from Deep Sea Drilling Project Leg 77, southeast Gulf of Mexico. In Buffler, R. T., Schlager, W., et al., Init. Repts. DSDP, 77: Washington (U.S. Govt. Printing Office), 649-674.

Wilcoxon, J. A., 1972. Upper Jurassic-Lower Cretaceous calcareous nannoplankton from the western North Atlantic Basin. In Hollister, C. D., Ewing, J. I., et al., Init. Repts. DSDP, 11: Washington (U.S. Govt. Printing Office), 221-225.

Wind, F. H., and Cepek, P., 1979. Lower Cretaceous calcareous nannoplankton from DSDP Hole 397A (northwest African Margin). In von Rad, U., and Ryan, W.B.F., et al., Init. Repts. DSDP, 47, Pt. 1: Washington (U.S. Govt. Printing Office), 269-492.

Wise, S. W., 1977. Mesozoic and Cenozoic calcareous nannofossils recovered by DSDP Leg 36 drilling on the Falkland Plateau, Southwest Atlantic Sector of the Southern Ocean. In Barker, P. Dalziel, I.W.D., et al., Init. Repts. DSDP, 36: Washington (U.S. Govt. Printing Office), 269-491.

Wise, S. W., and Wind, F., 1977. Mesozoic and Cenozoic calcareous nannofossils recovered by DSDP Leg 36 drilling on the Falkland Plateau, Southwest Atlantic sector of the Southern Ocean. In Barker, P., Dalziel, I.W.D., et al., Init. Repts. DSDP, 36: Washington (U.S. Govt. Printing Office), 269-492.

Worsley, T. R., 1971. Calcareous nannofossil zonation of Upper Jurassic and Lower Cretaceous sediments from the western Atlantic. Proc., Second Planktonic Conf. Roma 1970, 2:1301-1321.

Date of initial receipt: 22 January 1987

Date of acceptance: 10 August 1987

Ms 103B-144

\section{APPENDIX A \\ Systematic Paleontology \\ Genus COROLLITHION Stradner, 1961 \\ Corollithion acutum, Thierstein, 1972}

(Pl. 17, Figs. 4-6)

Ellipsochiastus quadriserratus Worsley, 1971, p. 1308, pl. 1, figs. 36-38 (invalid).

Corollithion acutum Thierstein, 1972, p. 438, pl. 2, figs. 1-9.

Corollithion acutum Thierstein, Covington and Wise, 1987, pl. 20, fig. 4.

Remarks. This small elliptical species of Corollithion is described by Thierstein (1972) as having " 40 to 50 non imbricate elements surmounted by a narrow rim." However, he illustrated a paratype (pl. 2, fig. 3) that has approximately 30 elements and in which the angle between the crossbars approaches $90^{\circ}$. Smaller specimens of this species were observed in this study and are constructed of 20 to 30 elements (Pl. 17, Figs. 5 and 6) whereas the larger, more rarer forms possess $40-50$ elements (Pl. 17, Fig. 4). This smaller form is believed to be the same as
Worsley's (1971) Ellipsochiastus quadriserratus and is considered here synonymous with Corollithion acutum. The angle between the crossbars in the larger forms is clearly less than $90^{\circ}$. In the smaller forms this angle approaches and sometimes reaches $90^{\circ}$.

Occurrences. Rare to few in the Valanginian through Aptian in ODP Holes 638B, 638C, and 641C. Worsley (1971) used the first occurrence of this species as a datum for the Berriasian/Valanginian boundary; however, we could not use it because of its rare and sporadic occurrence downhole.

\section{Genus CRUCIELLIPSIS Thierstein, 1971}

Cruciellipsis cuvillieri cuvillieri Thierstein, 1971

Cruciellipsis cuvillieri Thierstein, 1971, p. 477, pl. 5, figs. 4-8

Cruciellipsis cuvillieri ssp. grandis Applegate and Bergen, n. ssp. (Pl. 29, Figs. 9 and 10)

Diagnosis. A subspecies of Cruciellipsis cuvillieri distinguished by its small central area and robust size.

Description. The holotype is $12.5 \mu \mathrm{m}$ in length and the width of the central area is one-quarter or less the total width of the coccolith. Many specimens were observed that measure $15-16 \mu \mathrm{m}$ in length.

Differentiation. Cruciellipsis cuvillieri ssp. cuvillieri is usually less than $10 \mu \mathrm{m}$ and has a larger central area.

Remarks. Cruciellipsis cuvillieri ssp. grandis is common through 30 $\mathrm{m}$ of sediment in the lower part of Hole $638 \mathrm{C}$ and is found in layers of light greenish gray nannofossil marlstone containing moderately wellpreserved nannofossils that are not overgrown with calcite. Hence, the robust size and small central area are not a result of overgrowth.

Occurrences. Common in the lower Valanginian of ODP Hole 638C, Galicia margin. $2.5 \mu \mathrm{m}$.

Size. 11 to $16 \mu \mathrm{m}$; holotype $12.5 \mu \mathrm{m}$ in length. Central area, $3.5 \times$

Holotype. Plate 29, Figures 9 and 10.

Type locality. ODP Sample 103-638C-6R-1, 17-18 cm.

\section{Genus CYCLAGELOSPHAERA Noel, 1965}

Cyclagelosphaera brezae Applegate and Bergen, n. sp. (PI. 20, Figs. 1-3)

Diagnosis. A large delicate Cyclagelosphaera with a small proximal shield.

Description. A species of Cyclagelosphaera in which the diameter of the inner cycle is about one-third the total diameter of the coccolith. Both the proximal and distal shields contain approximately 30 flat, radial elements, and because of its large size, one can easily count the number of elements on the distal shield under the light microscope. In cross-polarized light, Cyclagelosphaera brezae exhibits a first-order gray to bright white birefringence. In sediments that contain overgrown nannofossils and are rich in calcite, $C$. brezae does not exhibit first-order colors in polarized light, as does Cyclagelosphaera deflandrei.

Remarks. Named in honor of marine sedimentologist James R. Breza, Florida State University. The large size and bright, but low-order, birefringence distinguish this taxon from other species of Cyclagelospaera.

Differentiation. This form differs from Cyclagelospaera margerelii by its larger size and less imbricate elements. Because of this difference in rim structure, $C$. margerelii appears brighter in polarized light.

Occurrences. Rare to common in the lower to upper Valanginian of ODP Holes 638B and 638C, Galicia margin.

Size. Holotype, $9.0 \mu \mathrm{m}$.

Holotype. Plate 20, Figures 1-3.

Type locality. ODP Sample 103-638B-44R, CC.

\section{Genus EIFFELLITHUS Reinhardt, 1965}

Eiffellithus primus Applegate and Bergen, n. sp. (Pl. 11, Figs. 1-12 and 14)

Diagnosis. A small species of Eiffellithus that has crossbars in the major and minor axes.

Description. Under the light microscope in cross-polarized light, this species exhibits split crossbars that are aligned with the major and mi- 
nor axes when viewed rotated $45^{\circ}$ to the crossed nicols. When viewed at $0^{\circ}$, the crossbars appear to be solid and appear to be at an angle from $10^{\circ}$ to $20^{\circ}$ from the major and minor axes. In phase contrast or transmitted light, the crossbars are observed in the major and minor axes.

Differentiation. Eiffellithus primus differs from Eiffellithus eximius by its smaller size and unique behavior under crossed polars (as previously described). Eiffellithus sp. 1 has its central cross $10^{\circ}-15^{\circ}$ from the major and minor axes and does not exhibit a split in the central cross in polarized light. It is distinguished from Eiffellithus windii $\mathrm{n}$. sp. by havings its crossbars in the major and minor axes.

Remarks. This species is the oldest known form of the genus Eiffellithus, and hence the name "primus". This form has its last occurrence near the first occurrence of $E$. windii, suggesting an evolutionary step in which the central cross rotates $45^{\circ}$ to the major and minor axes.

Occurrences. Rare to few in the lower to upper Valanginian of ODP Holes 638B, 638C, and 639A.

Size. Holotype, $6.5 \mu \mathrm{m}$ in length.

Holotype. Plate 11, Figures 1-5.

Type locality. ODP Sample $103-638 \mathrm{C}-10 \mathrm{R}-1,35-36 \mathrm{~cm}$.

\section{Eiffellithus windii, Applegate and Bergen, n. sp.} (Pl. 10, Figs. 1-6 and 8)

Eiffellithus? sp. Wind and Cepek, 1979, pl. 10, figs. 5-13.

Eiffellithus sp. 1. Covington and Wise, 1987, p. 34.

Diagnosis. A small species of Eiffellithus that has a small central area and short crossbars, intermediate to the major and minor axes. A stem may or may not be present.

Description. This small Eiffellithus has rather stubby crossbars that occupy most of the narrow, open central area, which is about one-half the total width of the coccolith. Under the SEM, the crossbars appear fibrous because of their construction of small parallel laths.

Differentiation. This form closely resembles Eiffellithus striatus (Black) and differs from the latter by its smaller size, narrower central opening, shorter crossbars, and the lack of split crossbars when observed in polarized light parallel to the crossed nicols. Transitional forms between these two species are observed, for which a size break of $6.4 \mu \mathrm{m}$ is used as the cutoff between them.

Remarks. Named in honor of Frank H. Wind, who, along with Pavel Cepek, first illustrated this species from the Hauterivian of Site 397, DSDP Leg 44. Their forms are smaller than the holotype, and in this section, a general decrease in size is seen downsection.

Occurrences. Rare to few in the mid-Valanginian to upper Hauterivian of ODP Holes $638 \mathrm{~B}$ and $638 \mathrm{C}$.

Size. Holotype, $5.8 \times 4.4 \mu \mathrm{m}$.

Holotype. Plate 10, Figures 1, 3, and 4.

Type locality. ODP Sample 103-638B-35R-3, 111-112 cm.

Eiffellithus striatus (Black) Applegate and Bergen, n. comb.

Chiastozygus striatus Black, 1971, pl. 34, Fig. 7.

Chiastozygus tripes Kothe, 1981, pl. 3, Figs. 4, 5.

Remarks. After examination of many specimens with both the light microscope SEM and existing illustrations in the literature, we conclude that this species belongs to the genus Eiffellithus. The rim structure differs from Chiastozygus and Tegumentum in having an inner cycle of plate-formed elements with a thin outer rim of highly inclined elements. Chiastozygus is constructed of a single cycle of elements with crossbars intermediate to the major and minor axis. The distinct elements of the inner cycle of Tegumentum are slightly inclined and imbricate, whereas the inner cycle of E. striatus resembles Eiffellithus; in most specimens this inner cycle appears granular (proximal view) or amorphous (distal view). It is difficult to discern any elements of the inner cycle from past illustrations, including those of the holotype (Black, 1971).

Genus HAYESITES Manivit, 1971, emend. Applegate, Covington, and Wise in Covington and Wise, 1987

Hayesites irregularis Thierstein, 1972, Applegate, Covington, and Wise in Covington and Wise, 1987

(Pl. 5, Figs. 1-12)

Rucinolithus irregularis Thierstein in Roth and Thierstein, 1972, p. 438, pl. 2, figs. 10-19.
Rucinolithus irregularis Thierstein, 1973, p. 45, pl. 3, figs. 1-14.

Hayesites irregularis (Thierstein) Applegate et al., 1986, p. 36.

Description. The observation of many specimens of this species allows a more complete description of Hayesites irregularis. The dextrally imbricate elements, seen clearly in distal view, form a low cone and emanate from a small inner ring of flat, slightly imbricate elements of the proximal side (Pl. 5, Figs. 2 and 12).

The inner ring of elements on the proximal side consists of about 24-28 flat, slightly dextral, imbricate elements that are indiscernable under the light microscope. Thierstein (1972) first described this species from specimens viewed from the distal side and later illustrated a proximal view (1973, pl. 3, fig. 11) in which this inner ring is faintly visible. The living form of this species probably attached the inner ring of the nannolith to the cell wall. This implies that the nannoliths would be concave outward, which is unlike the more common concave-inward structure of placoliths on coccospheres. However, the distal side of $H$. irregularis often exhibits a stem or central process, which one would expect to occur on the distal side of the nannolith.

Remarks. This species always exhibits a first-order white or gray birefringence, even in samples that are rich in carbonate and contain other nannofossils that are overgrown. Previous workers have extended the range of this species into the upper Barremian. The lowest occurrence of this species was observed only 2 cores below the magnetic event M0 (see "Magnetostratigraphy," "Site 641" chapter; Boillot, Winterer, et al., 1987), and the first-appearance datum of $H$. irregularis is accepted here as a valid datum for the Barremian/Aptian boundary.

\section{Hayesites albiensis Manivit, 1971 \\ (Pl. 4, Figs. 1-8)}

Hayesites albiensis Manivit, 1971, p. 138, pl. 14, figs. 1-7.

Hayesites albiensis Manivit, 1971, Thierstein, 1973, p. 45, pl. 6, figs. 15.

Remarks. Hayesites albiensis is rare to common in the lower Albian of ODP Hole $641 \mathrm{C}$. The last-appearance datum of this species was not observed because that part of the section was not recovered. An SEM micrograph (Pl. 4, Figs. 1-8) shows ornamentation on the distal side with radially extending pointed elements that bisect each of the larger proximal elements.

Genus LITHRAPHIDITES Deflandre, 1963

Lithraphidites bollii (Thierstein, 1971) Thierstein, 1973

$$
\text { (Pl. 14, Figs. 1-12) }
$$

Microrhabdulus bollii Thierstein, 1971, p. 481, pl. 3, figs. 6-10. Lithraphidites bollii (Thierstein) Thierstein, 1973, p. 45. Lithraphidites bollii (Thierstein) Thierstein, 1976, p. 350, pl. 3, figs. 24 26.

Remarks. Forms of Lithraphidites bollii, as illustrated in Thierstein $(1971,1976)$, are observed in ODP Holes 638B and 640A. This species seems to be restricted to the Tethyan region and is common in many samples of Hauterivian to early Barremian age. Forms questionably identified as Lithraphidites? bollii in Wind and Cepek (1979) are observed in samples from Hole 638C (Pl. 15, Figs. 4-6) and are early Valanginian in age. This form, as shown by an SEM micrograph, is clearly an overgrown Vekshinella parallela $\mathrm{n}$. comb., which has calcite crystals randomly oriented on the rhabdolith, unlike the orderly structure of $L$. bollii. Furthermore, if dissolution of a rhabdolith base and consequent overgrowth of the rhabdolith were the process necessary to "form" a $L$. bollii sensu stricto (Wind and Cepek, 1979), then one would expect to see intermediate forms in samples where both species are found. In samples from Hole 638B, with moderate to good preservation, both species are few to common and intermediate forms are not observed. It is concluded here that these are two separate and distinct species and that the first occurrence of $L$. bollii in the upper lower Hauterivian is a valid biostratigraphic datum.

\section{Genus NANNOCONUS Kamptner, 1931}

Nannoconus ligius Applegate and Bergen, n. sp.

(Pl. 13, Figs. 1-10 and 15)

Diagnosis. A small delicate Nannoconus that has eight distinct petaloid elements and a narrow canal. 
Description. This species is constructed of flat cycles with eight distinct petals emanating from the center, stacked upon one another to form eight columns parallel to the axis.

Remarks. Nannoconus ligius is easily distinguished from other taxa of the genus on end view by its eight distinct petals. The species was not observed in side view under the light microscope because it tends to orient its axis perpendicular to the field of view as a result of its short length. The canal, as observed with the SEM, is usually very narrow.

Differentiation. This species differs from Nannoconus cornuta in having straight parallel sides rather than a highly irregular shape. Nannoconus abundans has a flaring base and lacks the eight distinct elements of $N$. ligius.

Occurrences. Rare to few in the upper Hauterivian to lower Barremian in ODP Holes 638B and 640A.

Size. Holotype, $6 \mu \mathrm{m}$ in diameter.

Holotype. Plate 13, Figures 1 and 2.

Type locality. ODP Sample 103-638B-23R-5, 89-90 cm.

Genus PICKELHAUBE Applegate et al., 1987

Pickelhaube furtiva (Roth) Applegate et al., 1987

(Pl. 21, Figs. 1-12, and Pl. 22, Figs. 1, 3, and 9)

Cruciplacolithus furtivus Roth, 1983, p. 609, pl. 6, figs. 1-4.

Pickelhaube furtiva Applegate et al., 1987, p. 42, pl. 16, figs. 1 and 2, and pl. 23 , figs. 1 and 2 .

Remarks. The genus Pickelhaube, as described by Applegate et al. (1987), is a helmet-shaped nannofossil, consisting of a wide, flaring, nonimbricate proximal rim and a more distinct distal rim that yields an extinction figure reminiscent of that of the genus Reticulofenestra. The genus may or may not support a central multilath cross surmounted by a spine.

When viewed under the light microscope, this species exhibits a very high relief, as shown in Plate 21, Figures 2-5. Forms with a small central area are observed in younger sediments of Barremian and Aptian age. The species is rare in the lower Valanginian to the Barremian of ODP Holes 638B and 638C, with the exception of one sample. In Sample 103$641 \mathrm{C}-6 \mathrm{R}-3,123 \mathrm{~cm}$, taken within a coarse shell lag of broken shallowwater macrofossils, Pickelhaube furtiva was common and probably reworked from older sediments based on the presence of Calcicalathina oblongata in this sample. The nearshore provincalism of this species is indicated by the common occurrence of this species with the shallowwater nannofossil flora (as noted by Covington and Wise, 1987).

Pickelhaube sp. 1

(Pl. 22, Figs. 2, 4, and 7)

Remarks. This is a form of Pickelhaube that has an open central area. Many observed specimens of Pickelhaube lacked any central cross and process. In some of these specimens (Pl. 21, Figs. 7-12) the central process is lacking, probably because of preservation. However, it is clear that Pickelhaube sp. 1 in Plate 22, Figure 7, lacks a crossbar in structure, and the separation of this form is warranted. Pickelhaube sp. 1 closely resembles the Tertiary genus Reticulofenestra and can be differentiated from that genus by the former having much higher relief and a distinct distal ring of elements. The shape of Pickelhaube sp. 1 is variable; subrounded to elliptical forms are observed.

Occurrences. Rare to common in the lower Valanginian to lower Aptian of ODP Holes 638B, 638C, and 641C.

\section{Genus RETECAPSA Black, 1971 \\ Retecapsa angustiforata Black, 1971}

(Pl. 12, Figs. 3-6)

Retecapsa angustiforata Black, 1971, p. 409, pl. 33, fig. 4.

Retecapsa radiata (Worsley) Applegate and Bergen, n. comb. (PI. 12, Figs. 5 and 6)

Rucinolithus radiatus Worsley, 1971, pl. 1, figs. 51-52.

Hayesites bulbus Thierstein, 1972, p. 438, pl. 2, figs. 20-23.

Discoaster? atlanticus Wilcoxen, 1972, p. 431, pl. 6, figs. 5-6.

Remarks. This species was first described by Worsley (1971) who misreferenced it on p. 311 as plate 1 , figures 53 and 54 , whereas the figure captions were actually figures 51 and 52 . The name is therefore invalid, yet Thierstein (1976) validated it by using a new combination of Hayesites radiatus. In this study, it is shown that this species is clearly an ornament of Retecapsa angustiforata. However, Retecapsa radiata is not observed in sediment younger than Barremian and therefore, we choose to keep it a separate species because it has a useful stratigraphic range.

\section{Genus RUCINOLITHUS Stover, 1971}

Rucinolithus terebrodentarius Applegate et al., 1987

$$
\text { (Pl. 6, Figs. 1-8) }
$$

Rucinolithus terebrodentarius Applegate, Covington, and Wise, in Covington and Wise, 1987 , p. 48 , pl. 17, figs. $7-8$, pl. 18, figs. $5-7$, and pl. 19, figs. 1-5.

Remarks. This globular form of Rucinolithus resembles, under the light microscope, what could appear to be an overgrown $H$. irregularis. When viewed at different angles under the SEM, Rucinolithus terebrodentarius is globular and may revolve around an axis, whereas $H$. irregularis forms a low cone and always exhibits a low-order birefringence in polarized light, even in samples with abundant calcite and overgrown nannofossils. $R$. terebrodentarius has rare to few occurrences in the upper Barremian and is rare in the Aptian-lower Albian of ODP Holes 638B and 641C.

\section{Genus TUBODISCUS Thierstein, 1973}

Tubodiscus verenae, Thierstein, 1973

(Pl. 18, Fig. 9, Pl. 24, Figs. 3 and 6-9, Pl. 25, Figs. 1-10, and Pl. 26, Figs. 6-9)

Tubodiscus verenae Thierstein, 1973, pl. 2, figs. 1-7.

Remarks. Tubodiscus is described by Thierstein (1973) as having a central tube rising distally, producing a distinct collar. We found through the examination of many specimens under both the light microscope and SEM that this species exhibits at least a first-order yellow-orange birefringence under cross-polarized light, when viewed on end. There is an evolutionary change in this species from the lower Valanginian to the upper Hauterivian. In the lower Valanginian the collar is fully extended and is approximately $1.5 \times$ the total height of the coccolith (measured from the outer cycle of elements). When viewed in the light microscope under crossed nicols, on end view, the collar exhibits a first-order orange birefringence. The height of the collar decreases in younger forms and ultimately shortens to a height where it exhibits a first-order white under crossed nicols (see Tubodiscus jurapelagicus). Near the Valanginian/ Hauterivian boundary, the height of the collar is approximately onehalf the total height of the coccolith. At this height, a first-order yelloworange birefringence is still observed under polarized light. Previous workers have restricted this form to the Valanginian. However, this species occurs consistently up into the uppermost Hauterivian in Hole 638B, and therefore, its last occurrence is difficult to use as a datum for the Valanginian/Hauterivian boundary.

Occurrences. Lower Valanginian to upper Hauterivian of ODP Holes 638B and 638C.

\section{Tubodiscus jurapelagicus (Worsley) Roth, 1973}

$$
\text { (Pl. 24, Figs. } 1 \text { and 2) }
$$

Watznaueria jurapelagicus Worsley, 1971, p. 1315, pl. 2, figs. 29-31. Tubodiscus jurapelagicus Roth, 1973, p. 724, pl. 26, fig. 6. Tubodiscus sp. cf. T. verenae Wise and Wind, 1977, pl. 67, fig. 6.

Remarks. This form of Tubodiscus has a short distinct central collar and exhibits a first-order gray to white birefringence observed on end view under polarized light. This species is thought to have evolved from its close relative $T$. verenae as it is observed in younger sediment than the latter. However, the lowest occurrence of $T$. jurapelagicus below that of $T$. verenae would imply the opposite. Wise and Wind (1977) documented this species as $T$. verenae from Albian sediment from the Faukland Plateau. However, the central collar of the species they illustrated is not extended as in $T$. verenae. This species occurs from the lower Valanginian to upper Barremian in ODP Holes 638B, 638C, and $641 \mathrm{C}$.

\section{Genus VEKSHINELLA Loeblich and Tappan, 1963}

Vekshinella parallela (Wind and Cepek) Applegate and Bergen, $\mathrm{n}$. comb.

(Pl. 15, Figs. 1-9)

Eurhabdus luciformus Wilcoxon, 1972, pl. 10, fig. 4. 
Rhabdolekiskus parallelus Wind and Cepek, 1979, p. 232, pl. 3, figs. 3 6.

"Rhabdolekiskus" parallelus Covington and Wise, in press, pl. 23, figs. 3-5.

Remarks. This species is transferred to the genus Vekshinella based on the construction of the base of the rhabdolith as observed in SEM micrographs (Pl. 15, Figs. 8 and 9). The small base is constructed of a simple eiffellithid rim with a central cross aligned with the major and minor axes. When the species becomes overgrown, it vaguely resembles $L$. bollii; however, this was only observed in a few samples from the lower Valanginian. The length of $V$. parallela varies from $6-11 \mu \mathrm{m}$ and the base is $2-3 \mu \mathrm{m}$. Roth (1983) used the first-appearance datum of this species as a datum in the lower Hauterivian. The occurrence of this species in the bottom of Hole $638 \mathrm{C}$ extends the range of $V$. parallela down into the lower Valanginian.

Vekshinella pseudocarinolithus Applegate and Bergen n. sp. (Pl. 16, Figs. 1-9)

Rhabdolith with possible affinity to Diadorhombus rectus Worsley, Wind and Cepek, 1979, explanation for pl. 3, figs. 7 and 8 .

Genus et species indet. 2, Covington and Wise, 1987, p. 56, pl. 23, figs. 7 and 8 .

Diagnosis. Straight-sided rhabdolith with a small Vekshinella base at the proximal end and a large "flowery" ornament on the distal end.

Description. The base of this rhabdolith is constructed of a simple eiffellithal rim with a central cross aligned in the major and minor axes. The distal end is constructed of about six large "misshapened" elements.

Differentiation. In side view, this species closely resembles those assigned to the genus Carinolithus from the Jurassic. Carinoliths have bases with lamellar radial elements at a low angle, whereas this species has a Vekshinella base. The distal ornament of this species resembles the species Hexalithus hexalithus, which measures about $4 \mu \mathrm{m}$ and was described from the Upper Jurassic (Covington and Wise, 1987). Wiegand (1984, pl. 1, figs. 1-4) illustrated another form Hexalithus magharensis, which possesses a stem. W. A. Bergen (unpubl. data) has observed that H. magharensis is actually distal parts on Carinolithus; thus, it appears that Hexalithus may represent a form genus.

Remarks. This species is named for its close resemblance to the genus Carinolithus.

Occurrences. Rare to few in the lower Valanginian to Hauterivian of ODP Holes 638C and 638B.

Size. Holotype, base $2.2 \mu \mathrm{m}$, length $9.5 \mu \mathrm{m}$.

Holotype. Plate 16, Figures 8 and 9.

Type locality. ODP Sample 103-638B-45R-3, $28-29 \mathrm{~cm}$.

Vekshinella mitcheneri Applegate and Bergen n. sp. (Pl. 23, Figs. 7-9)

Eiffellithus? sp. 2 Covington and Wise, 1987, p. 34, pl. 22, figs. 7-9.

Diagnosis. A form of Vekshinella that has a small central area that exhibits a bright birefringence under crossed polars.

Description. The distal rim of this species is constructed of highly inclined, imbricate elements that give this species a bright birefringence under crossed polars. The ends of the central cross flare at the rim, resulting in a small central openings. The crossbars exhibit a bright, white birefringence.

Remarks. This species resembles the genus Eiffellithus. However, in comparison to that species (Pl. 11), one can clearly observe the difference in the rim structure. Eiffellithus has a wider inner cycle and a thinner outer rim structure. The central cross of $E$. primus exhibits different optical characteristics in polarized light than does Vekshinella mitcheneri. It differs from Vekshinella stradneri in having a much brighter birefringence in polarized light and crossbars with flared distal extremities.

Remarks. Although many of the observed forms are small ( $\sim 4-5$ $\mu \mathrm{m})$, their size increases in the upper Hauterivian-lower Barremian. Covington and Wise (1987) described this species as Eiffellithus? sp. 2 from the Barremian and illustrated a form that is larger than the holotype.

Occurrences. Rare to few in the lower Valanginian to upper Barremian of ODP Holes $638 \mathrm{~B}, 638 \mathrm{C}$, and $639 \mathrm{~A}$.

Size. Holotype, $4.1 \mu \mathrm{m}$ in length.

Holotype. Plate 23, Figures 7-9.

Type locality. ODP Sample 103-638B-30R-5, 5-6 cm.
Genus ZEUGRHABDOTUS Reinhardt, 1965

Zeugrhabdotus pseudoangustus Bralower, Applegate, Covington, and Wise, in Wise and Covington, 1987

(Pl. 7, Figs. 11 and 12)

Rhagodiscus cf. R. angustus Perch-Nielsen, 1979, p. 229, fig. 3.

Zeugrhabdotus? pseudoangustus Bralower, Applegate, Covington, and Wise, in Covington and Wise, 1987, p. 53, pl. 8, figs. 2-4.

Remarks. This species, which closely resembles Rhagodiscus angustus, is rare to common in many samples from ODP Holes 638B and $638 \mathrm{C}$. A proximal or basal granular cover has not been observed across the rim as in $R$. angustus. Zeugrhabdotus pseudoangustus can be distinguished from $R$. angustus by the former's lack of a basal cover, a smaller length to width ratio, and a subrectangular outline with somewhat squared ends. The bridge that spans the minor axis is delicate, and in most specimens, not observed. Z. pseudoangustus is rare to common in the lower Valanginian to upper Barremian to ODP Holes 638B and $638 \mathrm{C}$.

\section{APPENDIX B \\ Calcareous Nannofossils Considered in This Chapter (in alphabetical order of generic epithets)}

Amphizygus brooksii Bukry, 1969

Assipetra infracretacea (Thierstein) Roth, 1973

Axopodorhabdus albianus (Black) Wind and Wise in Wise and Wind, 1977

Axopodorhabdus dietzmannii (Reinhardt) Wind and Wise, 1983

Biscutum constans (Gorka) Black in Black and Barns, 1959

Braarudosphaera africana Stradner, 1961

Braarudosphaera discula Bramlette and Riedel, 1954

Bukrylithus ambiguus Black, 1971a

Calcicalathina oblongata (Worsley) Thierstein, 1971

Chiastozygus litterarius (Gorka) Manivit, 1971

Chiastozygus platyrhethus Hill, 1976

Chiastozygus tenuis Black, 1971

Conusphaera mexicana Trejo, 1969

Corollithion achylosum (Stover) Thierstein, 1971

Corollithion acutum Thierstein in Roth and Thierstein, 1972

Corollithion ellipticum Bukry, 1969

Cretarhabdus conicus Bramlette and Martini, 1964

Cretarhabdus delicatus Applegate, Covington, and Wise in Covington and Wise, 1987

Cretarhabdus cf. loriei Gartner, 1969

Cretarhabdus loriei Gartner, 1969

Cretarhabdus surirellus (Deflandre in Deflandre and Fert) Reinhardt emend. Thierstein, 1971

Cribrosphaerella ehrenbergii (Arkhangelsky) Deflandre in Piveteau, 1952

Crucicribrum striatum striatum (Stradner) Wise, 1983

Cruciellipsis cuvillieri cuvillieri (Manivit) Thierstein, 1971

Cruciellipsis cuvillieri ssp. grandis Applegate and Bergen, n. ssp.

Cyclagelosphaera brezae Applegate and Bergen, n. sp.

Cyclagelosphaera deflandrei (Manivit) Roth, 1973

Cyclagelosphaera margerelii Noel, 1965

Cylindralithus nudus Bukry, 1969

Darwinilithus pentarhethum Watkins in Watkins and Bowdler, 1984

Diadorhombus rectus Worsley, 1971

Diazomatolithus lehmanii Noel, 1965

Diloma placinum Wind and Cepek, 1979

Diloma primitiva (Worsley) Wind and Cepek, 1979

Discorhabdus biradiatus (Worsely) Thierstein, 1973

Discorhabdus ignotus (Gorka) Perch-Nielsen, 1968

Discorhabdus sp. 1

Eiffellithus primus Applegate and Bergen, n. sp.

Eiffellithus striatus (Black) Applegate and Bergen, n. comb.

Eiffellithus turriseiffeli (Deflandre in Deflandre and Fert) Reinhardt, 1965

Eiffellithus windii Applegate and Bergen, n. sp.

Eiffellithus sp. 1 Perch-Nielsen, 1979

Eiffellithus sp. 2 Perch-Nielsen, 1979

Eiffellithus sp. 3 Perch-Nielsen, 1979

Ellipsagelosphaera ovata (Bukry) Black, 1973

Eprolithus floralis (Stradner) Stover, 1966

Ethmorhabdus hauterivianus (Black) Applegate, Covington, and Wise in Covington and Wise, 1987 
Flabellites biforaminis Thierstein, 1973

Flabellites oblongus (Bukry) Crux in Crux et al., 1982

Glaukolithus diplogrammus (Deflandre in Deflandre and Fert) Reinhardt, 1964

Grantarhabdus coronadventis (Reinhardt) Grun in Grun and Allemann, 1975

Grantarhabdus meddii Black, 1971

Haqius circumradiatus (Stover) Roth, 1978

Hayesites albiensis Manivit, 1971

Hayesites irregularis (Thierstein) Applegate, Covington, and Wise in Covington and Wise, 1987

Helicolithus trabeculatus (Gorka) Verbeek, 1977b

Lapideacassis sp.

Lithraphidites acutus acutus Verbeek and Manivit in Manivit et al., 1977

Lithraphidites acutus eccentricus Watkins in Watkins and Bowdler, 1984

Lithraphidites alatus Thierstein in Roth and Thierstein, 1972

Lithraphidites bollii (Thierstein) Thierstein, 1973

Lithraphidites carniolensis Deflandre, 1963

Manivitella cf. pecten Black, 1973

Manivitella pemmatoidea (Deflandre in Manivit) Thierstein, 1971

Micrantholithus hoschulzii (Reinhardt) Thierstein, 1971

Micrantholithus obtusus Stradner, 1963

Microstaurus chiastius (Worsley) Grun in Grun and Allemann, 1975

Microstaurus conus (Worsley) Wind and Cepek, 1979

Nannoconus abundans Stradner and Grun, 1973

Nannoconus bermudezii Bronnimann, 1955

Nannoconus bucheri Bronnimann, 1955

Nannoconus elongatus Bronnimann, 1955

Nannoconus globulus Bronnimann, 1955

Nannoconus grandis Deres and Acheriteguy, 1980

Nannoconus ligius Applegate and Bergen, n. sp.

Nannoconus kamptneri Bronnimann, 1955

Nannoconus steinmannii Kamptner, 1931

Nannoconus truitti Deres and Acheriteguy, 1980

Nannoconus wassallii Bronnimann, 1955

Nodosella silvaradion (Filewicz et al. in Wise and Wind) Perch-Nielsen, 1984

Octopodorhabdus plethotretus Wind and Cepek ex Applegate, Covington, and Wise in Covington and Wise, 1987

Parhabdolithus judithae Black, 1972

Percivalia fenestrata (Worsley) Wise, 1983

Perissocyclus noeliae Black, 1971, emend. Wind and Cepek, 1979

Pickelhaube furtiva (Roth) Applegate, Covington, and Wise in Covington and Wise, 1987

Pickelhaube sp. 1

Prediscosphaera columnata (Stover) Perch-Nielsen, 1984

Prediscosphaera cretacea (Arkangelsky) Gartner, 1968

Prediscosphaera spinosa (Bramlette and Martini) Gartner, 1968

Radiolithus planus Stover, 1966

Retecapsa angustiforata Black, 1971a

Retecapsa neocomiana Black, 1971a

Retecapsa radiata (Worsley) Applegate and Bergen, n. comb

Rhagodiscus angustus (Stradner) Reinhardt, 1971

Rhagodiscus asper (Stradner) Reinhardt, 1967

Rhagodiscus infinitus (Worsley) Applegate, Covington, and Wise in Covington and Wise, 1987

Rhagodiscus splendens (Deflandre) Verbeek, 1977

Rhagodiscus swinnertonii (Black) Applegate, Covington, and Wise, in Covington and Wise, 1987

Rhombolithion rhombicum (Stradner and Adamiker) Black, 1973

Rucinolithus terebrodentarius Covington and Wise, 1987

Rucinolithus wisei Thierstein, 1971

Sollasites hayi (Black) Perch-Nielsen, 1984

Sollasites horticus (Stradner et al. in Stradner and Adamiker) Cepek and Hay, 1969

Sollasites lowei (Bukry) Roth, 1970

Speetonia colligata Black, 1971

Stephanolithion laffittei Noel, 1956

Tegumentum stadneri Thierstein in Roth and Thierstein, 1972

Tetrapodorhabdus coptensis Black, 1971

Tetrapodorhabdus decorus (Deflandre in Deflandre and Fert) Wind and Wise in Wise and Wind, 1977
Thurmannolithion clatratum Grun and Zweili, 1980

Tranolithus exiguus Stover, 1977

Tranolithus gabalus Stover, 1966

Tubodiscus verenae Thierstein, 1973

Tubodiscus jurapelagicus (Worsley) Roth, 1973

Vagalapilla matalosa (Stover) Thierstein, 1973

Vekshinella angusta (Stover) Verbeek, 1977b

Vekshinella mitcheneri Applegate and Bergen, n. sp.

Vekshinella parallela (Wind and Cepek) Applegate and Bergen, n. comb.

Vekshinella pseudocarinolithus Applegate and Bergen, n. sp.

Vekshinella stradneri Rood, Hay, and Barnard, 1971

Watznaueria barnesae (Black in Black and Barnes) Perch-Nielsen, 1968

Watznaueria biporta Bukry, 1969

Watznaueria britannica (Stradner) Reinhardt, 1964

Zeugrhabdotus embergeri (Noel) Perch-Nielsen, 1984

Zeugrhabdotus pseudoangustus bralower, Applegate, Covington, and Wise in Covington and Wise, 1987

Zeugrhabdotus sp. (small)

Zeugrhabdotus theta (Black in Black and Barnes) Black, 1973

\section{APPENDIX C \\ Calcareous Nannofossils Considered in This Chapter (in alphabetical order of specific epithets)}

Nannoconus abundans Stradner and Grun, 1973

Corollithion achylosum (Stover) Thierstein, 1971

Corollithion acutum Thierstein in Roth and Thierstein, 1972

Lithraphidites acutus acutus Verbeek and Manivit in Manivit et al., 1977

Lithraphidites acutus eccentricus Watkins in Watkins and Bowdler, 1984

Braarudosphaera africana Stradner, 1961

Lithraphidites alatus alatus Thierstein in Roth and Thierstein, 1972

Axopodorhabdus albianus (Black) Wind and Wise in Wise and Wind, 1977

Hayesites albiensis Manivit, 1971

Bukrylithus ambiguus Black, 1971a

Vekshinella angusta (Stover) Verbeek, 1977b

Retecapsa angustiforata Black, 1971a

Rhagodiscus angustus (Stradner) Reinhardt, 1971

Rhagodiscus asper (Stradner) Reinhardt, 1967

Watznaueria barnesae (Black in Black and Barnes) Perch-Nielsen, 1968

Nannoconus bermudezii Bronnimann, 1955

Flabellites biforaminis Thierstein, 1973

Watznaueria biporta Bukry, 1969

Discorhabdus biradiatus (Worsley) Thierstein, 1973

Lithraphidites bollii (Thierstein) Thierstein, 1973

Cyclagelosphaera brezae Applegate and Bergen, n. sp.

Watznaueria britannica (Stradner) Reinhardt, 1964

Nannoconus bucheri Bronnimann, 1955

Lithraphidites carniolensis Deflandre, 1963

Microstaurus chiastius (Worsley) Grun in Grun and Allemann, 1975

Haqius circumradiatus (Stover) Roth, 1978

Thurmannolithion clatratum Grun and Zweili, 1980

Speetonia colligata Black, 1971

Prediscosphaera columnata (Stover) Perch-Nielsen, 1984

Cretarhabdus conicus Bramlete and Martini, 1964

Biscutum constans (Gorka) Black in Black and Barns, 1959

Microstaurus conus (Worsley) Wind and Cepek, 1979

Tetrapodorhabdus coptensis Black, 1971

Grantarhabdus coronadventis (Reinhardt) Grun in Grun and Allemann, 1975

Prediscosphaera cretacea (Arkangelsky) Gartner, 1968

Cruciellipsis cuvillieri cuvillieri (Manivit) Thierstein, 1971

Cruciellipsis cuvillieri ssp. grandis Applegate and Bergen, n. ssp.

Tetrapodorhabdus decorus (Deflandre in Deflandre and Fert) Wind and Wise in Wise and Wind, 1977

Cyclagelosphaera deflandrei (Manivit) Roth, 1973

Cretarhabdus delicatus Applegate, Covington, and Wise in Covington and Wise, 1987

Axopodorhabdus dietzmannii (Reinhardt) Wind and Wise, 1983

Glaukolithus diplogrammus (Deflandre in Deflandre and Fert) Reinhardt, 1964

Braarudosphaera discula Bramlette and Riedel, 1954 
Cribrosphaerella ehrenbergii (Arkangelsky) Deflandre in Piveteau, 1952 Corollithion ellipticum Bukry, 1969

Nannoconus elongatus Bronnimann, 1955

Zeugrhabdotus embergeri (Noel) Perch-Nielsen, 1984

Tranolithus exiguus Stover, 1977

Percivalia fenestrata (Worsley) Wise, 1983

Eprolithus floralis (Stradner) Stover, 1966

Pickelhaube furtiva (Roth) Applegate, Covington, and Wise, in Covington and Wise, 1987

Tranolithus gabalus Stover, 1966

Nannoconus globulus Bronnimann, 1955

Nannoconus grandis Deres and Acheriteguy, 1980

Ethmorhabdus hauterivianus (Black) Applegate, Covington, and Wise in Covington and Wise, 1987

Sollasites hayi (Black) Perch-Nielsen, 1984a

Sollasites horticus (Stradner et al. in Stadner and Adamiker) Cepek and Hay, 1969

Micrantholithus hoschulzii (Reinhardt) Thierstein, 1971

Discorhabdus ignotus (Gorka) Perch-Nielsen, 1968

Rhagodiscus infinitus (Worsley) Applegate, Covington, and Wise in Covington and Wise, 1987

Assipetra infracretacea (Thierstein) Roth, 1973

Hayesites irregularis (Thierstein) Applegate, Covington, and Wise in Covington and Wise, 1987

Parhabdolithus judithae Black, 1972

Tubodiscus jurapelagicus (Worsley) Roth, 1973

Nannoconus kamptneri Bronnimann, 1955

Stephanolithion laffittei Noel, 1956

Diazomatolithus lehmanii Noel, 1965

Nannoconus ligius Applegate and Bergen, n. sp.

Chiastozygus litterarius (Gorka) Manivit, 1971

Cretarhabdus loriei Gartner, 1969

Cretarhabdus sp. cf. C. loriei Gartner, 1969

Sollasites lowei (Bukry) Roth, 1970

Cyclagelosphaera margerelii Noel, 1965

Vagalapilla matalosa (Stover) Thierstein, 1973

Grantarhabdus meddii Black, 1971

Conusphaera mexicana Trejo, 1969

Vekshinella mitcheneri Applegate and Bergen, n. sp.

Retecapsa neocomiana Black, 1971a

Perissocyclus noeliae Black, 1971, emend. Wind and Cepek, 1979

Cylindralithus nudus Bukry, 1969

Calcicalathina oblongata (Worsley) Thierstien, 1971

Flabellites oblongus (Bukry) Crux in Crux et al., 1982

Micrantholithus obtusus Stradner, 1963

Ellipsagelosphaera ovata (Bukry) Black, 1973

Vekshinella parallela (Wind and Cepek) Applegate and Bergen, n. comb.
Manivitella sp. cf. M. pecten Black, 1973

Manivitella pemmatoidea (Deflandre in Manivit) Thierstein, 1971

Darwinilithus pentarhethum Watkins in Watkins and Bowdler, 1984

Diloma placinum Wind and Cepek, 1979

Radiolithus planus Stover, 1966

Chiastozygus platyrhethus Hill, 1976

Octopodorhabdus plethotretus (Wind and Cepek) Applegate, Covington, and Wise, in Covington and Wise, 1987

Diloma primitiva (Worsley) Wind and Cepek, 1979

Eiffellithus primus Applegate and Bergen, n. sp.

Zeugrhabdotus pseudoangustus Bralower, Applegate, Covington, and Wise in Covington and Wise, 1987

Vekshinella pseudocarinolithus Applegate and Bergen, n. sp.

Retecapsa radiata (Worsley) Applegate and Bergen, n. comb

Diadorhombus rectus Worsley, 1971

Rhombolithion rhombicum (Stradner and Adamiker) Black, 1973

Nodosella silvaradion (Filewicz et al. in Wise and Wind) Perch-Nielsen, 1984

Discorhabdus sp. 1

Eiffellithus sp. 1 Perch-Nielsen, 1979

Eiffellithus sp. 2 Perch-Nielsen, 1979

Eiffellithus sp. 3 Perch-Nielsen, 1979

Lapideacassis $\mathrm{sp}$.

Pickelhaube sp. 1 Applegate and Bergen

Prediscosphaera spinosa (Bramlette and Martini) Gartner, 1968

Rhagodiscus splendens (Deflandre) Verbeek, 1977

Nannoconus steinmannii Kamptner, 1931

Tegumentum stradneri Thierstein in Roth and Thierstein, 1972

Vekshinella stradneri Rood, Hay, and Barnard, 1971

Crucicribrum striatum striatum (Stradner) Wise, 1983

Eiffellithus striatus (Black) Applegate and Bergen, n. comb.

Creatarhabdus surirellus (Deflandre in Deflandre and Fert) Reinhardt emend. Thierstein, 1971

Rhagodiscus swinnertonii (Black) Applegate, Covington, and Wise in Covington and Wise, 1987

Chiastozygus tenuis Black, 1971

Rucinolithus terebreodentarius Covington and Wise, 1987

Zeugrhabdotus theta (Black in Black and Barns) Black, 1973

Helicolithus trabeculatus (Gorka) Verbeek, 1977b

Nannoconus truitti Deres and Acheriteguy, 1980

Eiffellithus turriseiffeli (Deflandre in Deflandre and Fert) Reinhardt, 1965

Tubodiscus vereane Thierstein, 1973

Nannoconus wassallii Bronnimann, 1955

Eiffellithus windii Applegate and Bergen, n. sp.

Rucinotithus wisei Thierstein, 1971

\section{NOTE ON PLATES}

The illustrations on the following plates are scanning electron and light micrographs. The abbreviations $\mathrm{P}, \mathrm{D}$, and $\mathrm{L}$ denote proximal, distal, and lateral views, respectively. The abbreviations $\mathrm{Pol}, \mathrm{Ph}$, and $\mathrm{Tr}$ denote light micrographs that were taken in cross-polarized, phase contrast, and transmitted light, respectively. CN is an abbreviation for crossed nicols. A photomicrograph of a specimen that is also illustrated by a SEM micrograph is denoted in the plate captions as a superscript on the photomicrograph illustration number. For example, on Plate $1,(2 \text { and } 3)^{\text {s1 }}$ denotes that the specimen shown in light micrographs 2 and 3 is the same specimen illustrated in the SEM micrograph figure 1. 




1

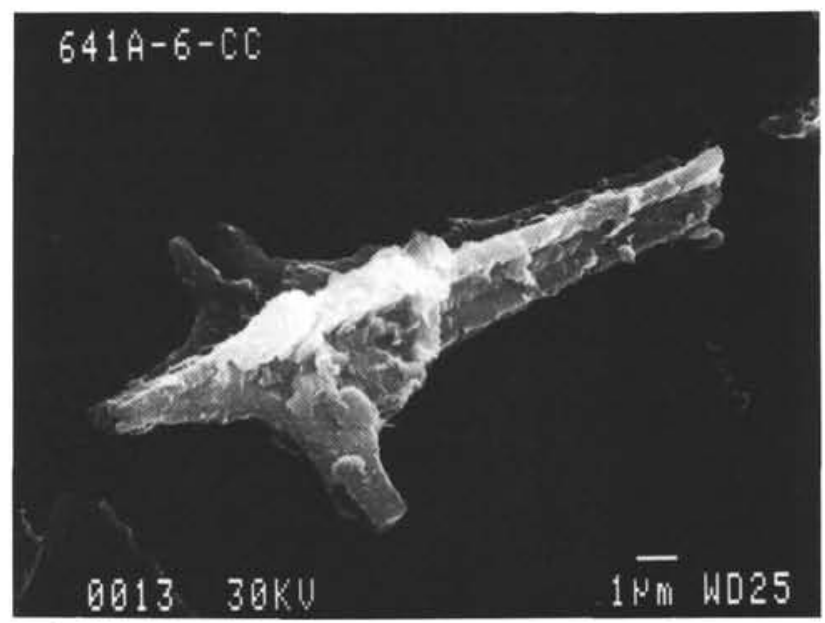

6

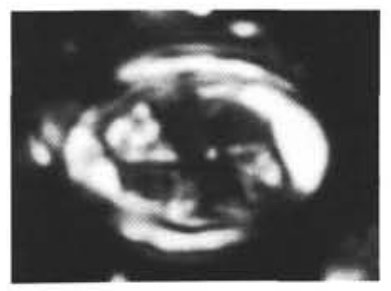

8

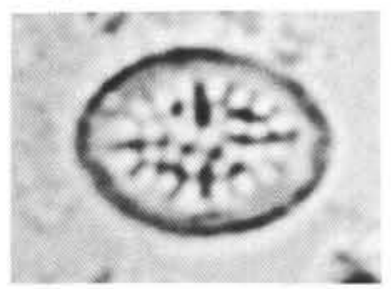

10

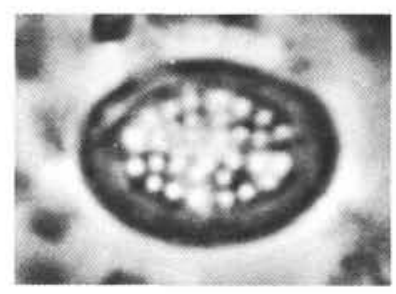

9

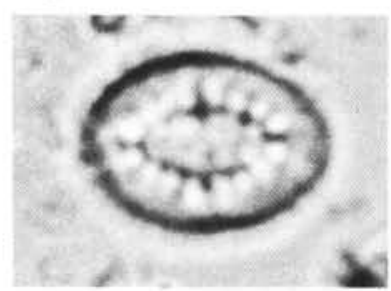

11



2

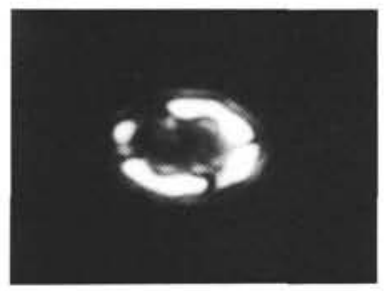

4

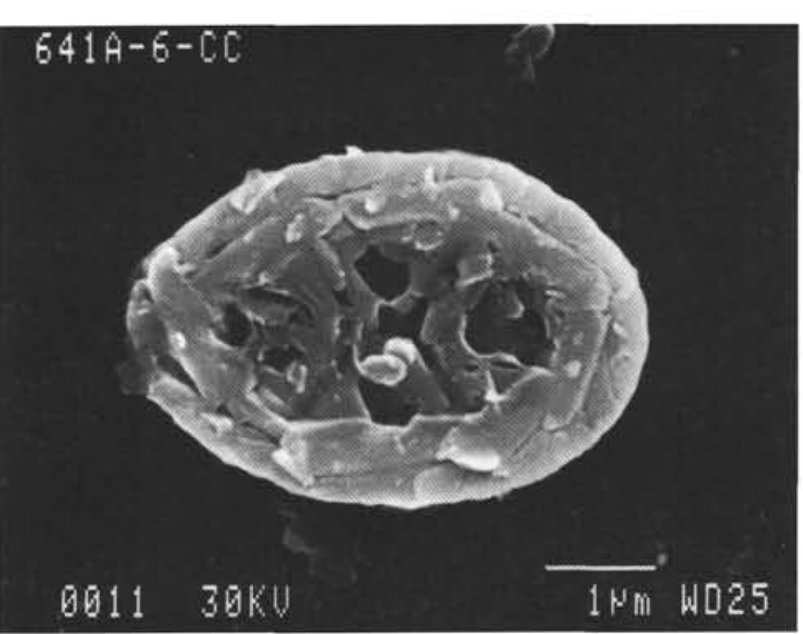

7

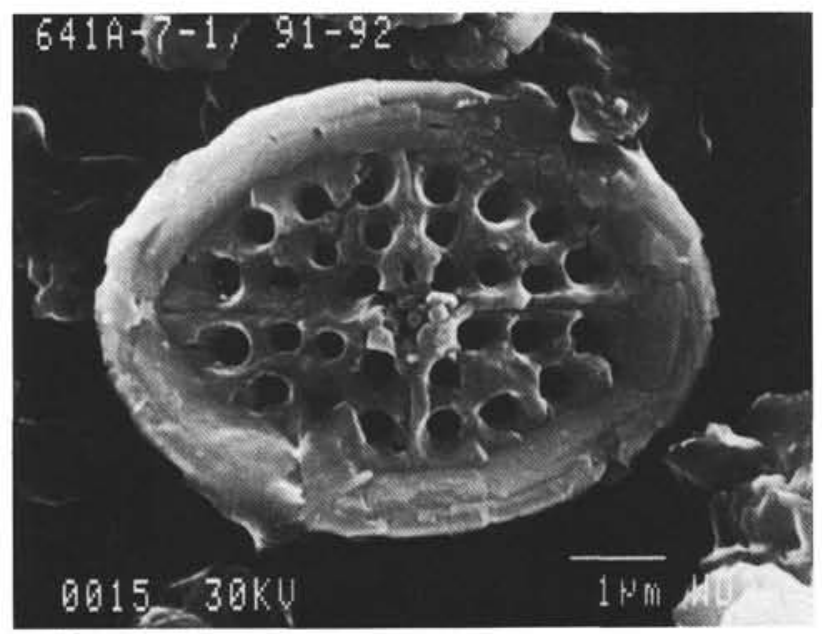

12

Plate 1. 1-3 and 6. Lithraphidites acutus ssp. eccentricus Watkins, Sample $103-641 \mathrm{~A}-6 \mathrm{X}, \mathrm{CC},(1 \mathrm{~cm}), \mathrm{L}, 3400 \times,(2 \text { and } 3)^{\mathrm{s1}} 1600 \times(2$, Tr, high focus; 3, Tr, low focus). 4, 5 and 7. Helicolithus trabeculatus (Gorka), Sample 103-641A-6X, CC, (4 and 5) ${ }^{57} 3500 \times(4, \mathrm{Pol} ; 5, \mathrm{Ph}),(7) \mathrm{D}$, $11,000 \times$. 8-12. Crucicribrum striatum ssp. striatum Stradner, Sample 641A-7X-1, 91-92 cm (8-11) $)^{\mathrm{s} 12} 4000 \times,(8, \mathrm{Pol} ; 9, \mathrm{Ph} ; 10$, Tr, low focus; 11, $\mathrm{Tr}$, high focus), (12), D, $9000 \times$. 


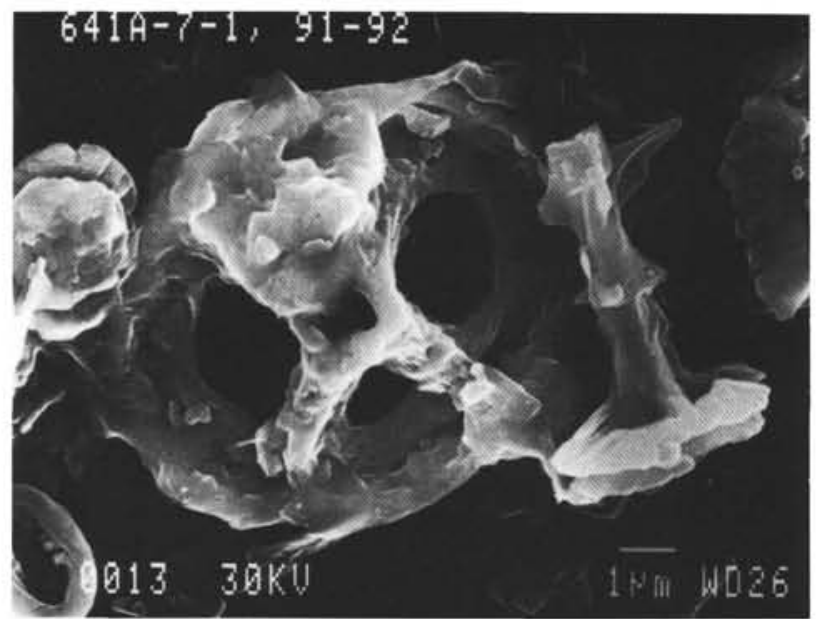

1

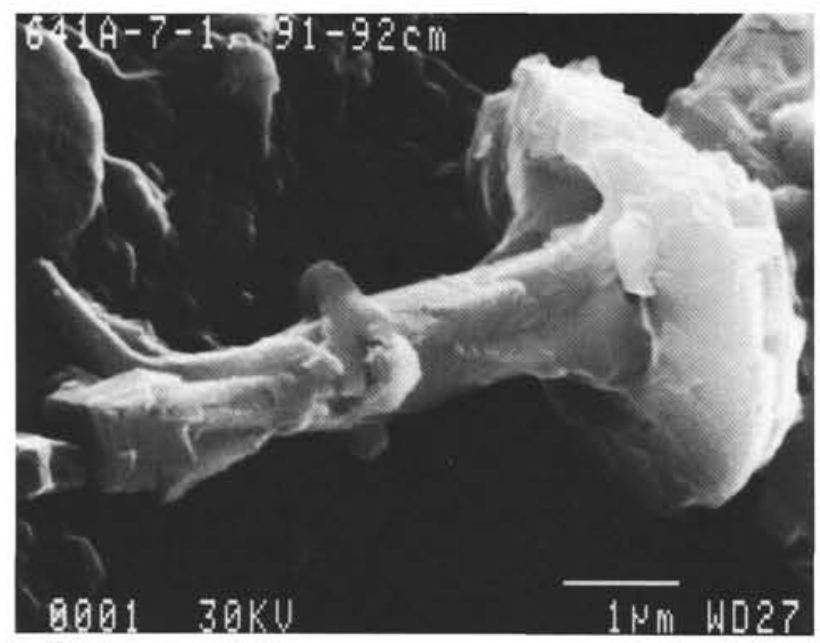

6

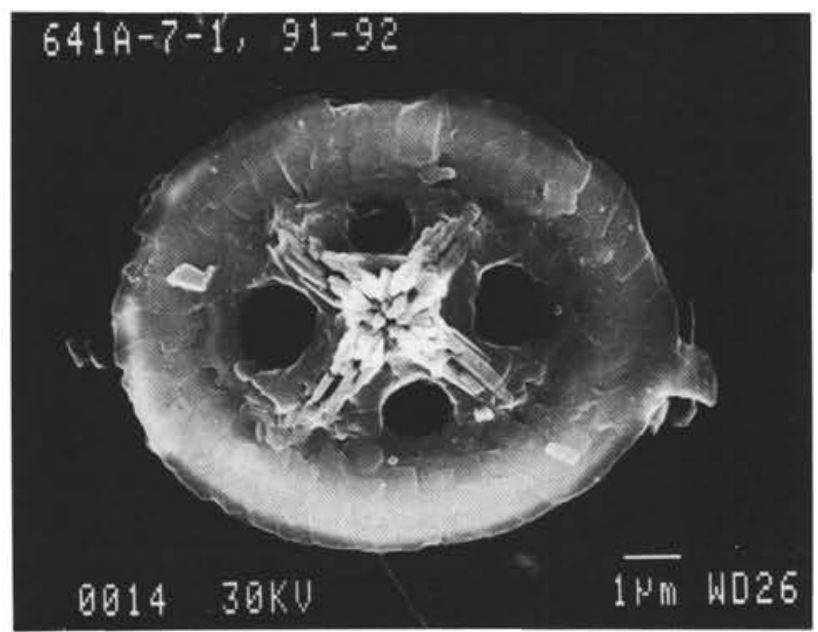

8

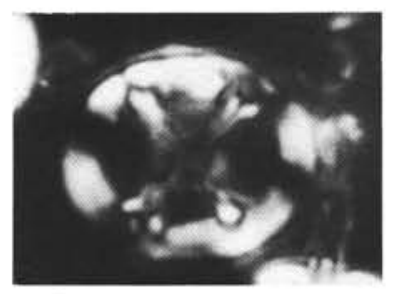

2

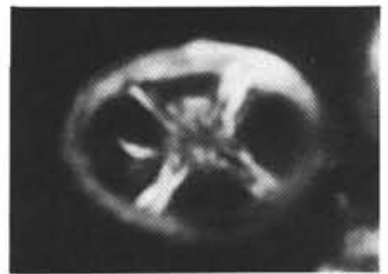

4

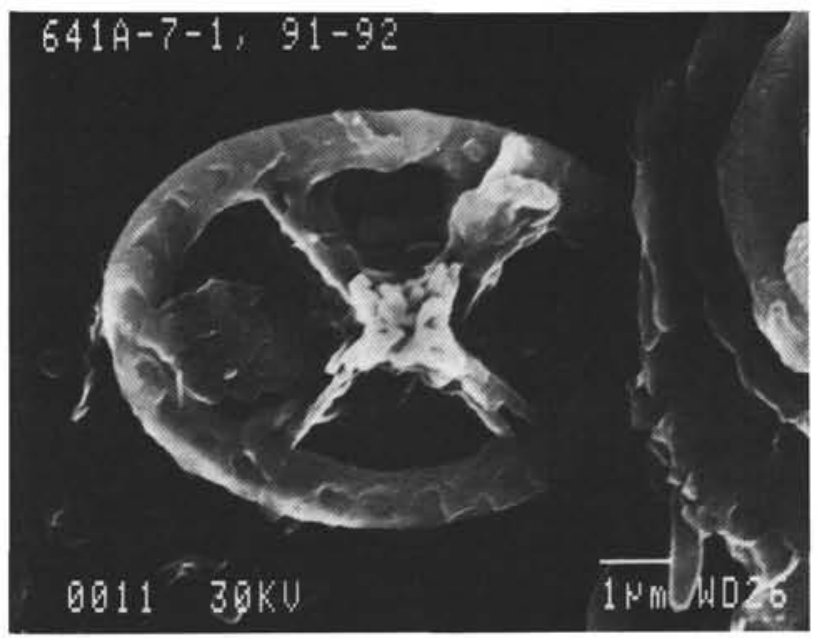

7

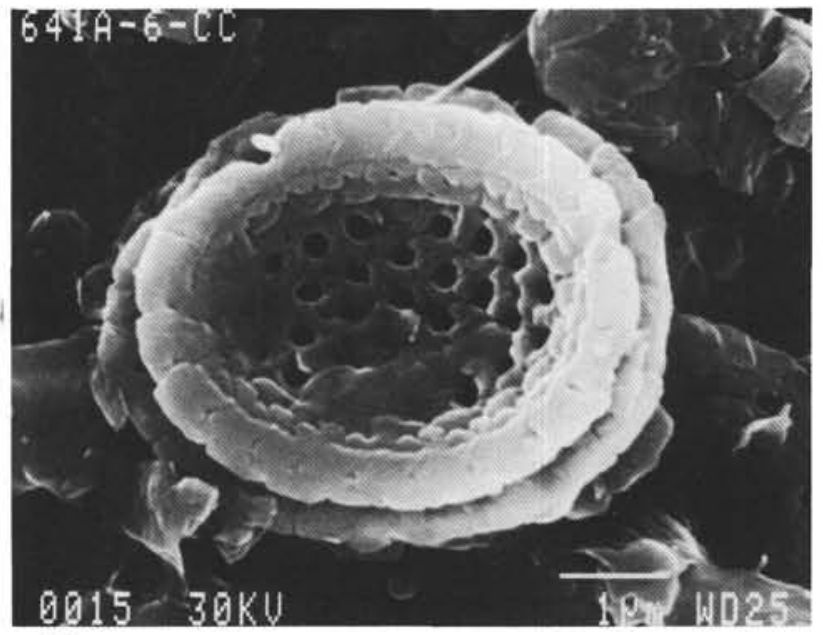

9

Plate 2. 1-3. Eiffellithus turriseiffeli (Deflandre) (on left, D) and Prediscosphaera columnata (Stover) (on right, L), Sample 103-641A-7X-1, 91-92 $\mathrm{cm},(1) 5500 \times,(2 \text { and } 3)^{\mathrm{s1}} 3400 \times,(2, \mathrm{Pol}: 3, \mathrm{Ph}) .4,5$ and 7 . Chiastozygus platyrhethum Hill, Sample 103-641A-7X-1, 91-92 cm, (4 and 5) ${ }^{57}$ $3400 \times,(4, \mathrm{Pol} ; 5, \mathrm{Ph}),(7) \mathrm{D}, 7100 \times$. 6. Prediscosphaera spinosa (Bramlette and Martini), Sample 103-641A-7X-1, 91-92 cm, L, 12,000×. 8. Grantarhabdus coronadventis (Reinhardt), Sample 103-641A-7X-1, 91-92 cm, D, 5800×. 9. Cribrosphaerella ehrenbergii (Arkangelsky), Sample 103-641-6X, CC, P, 11,200x. 

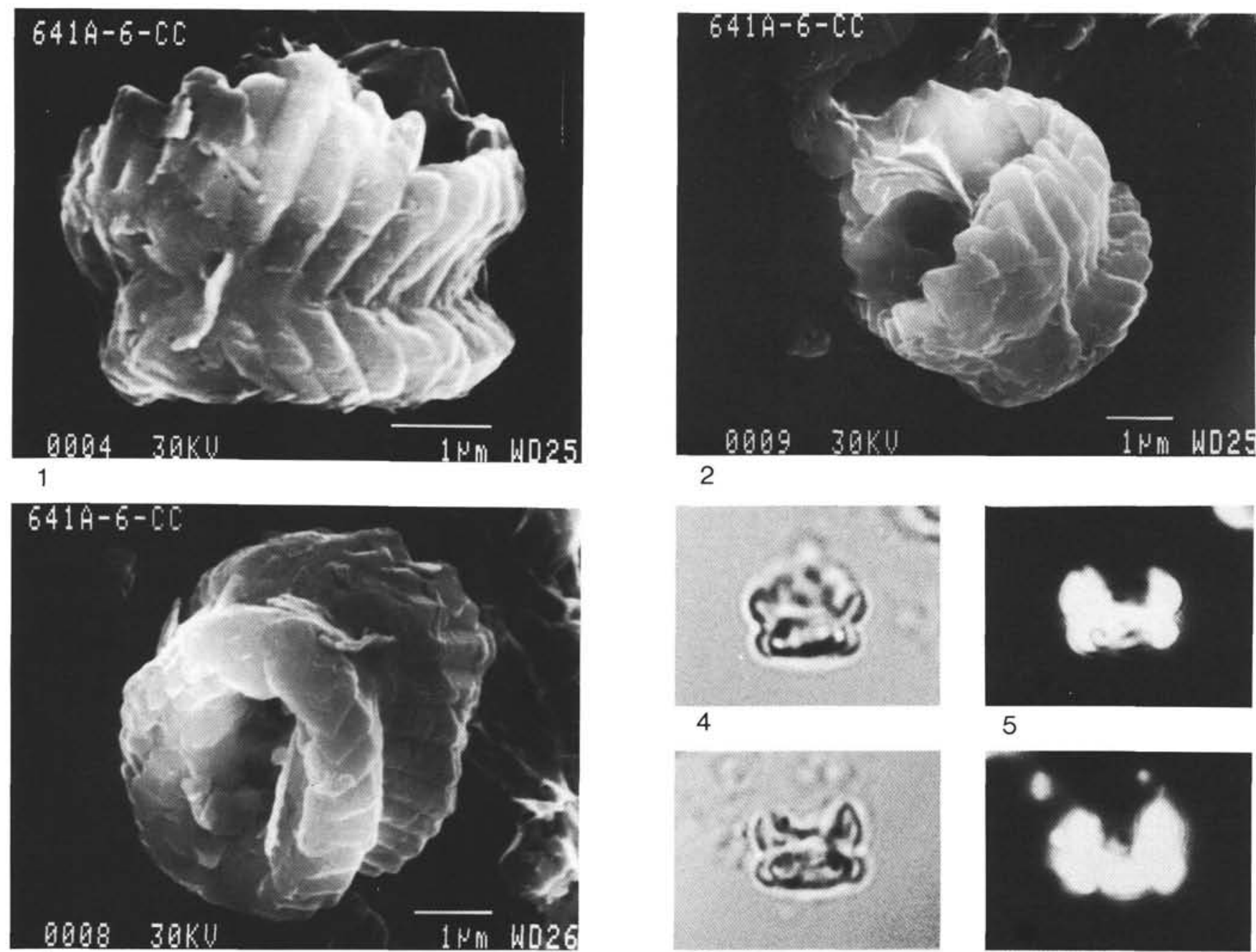

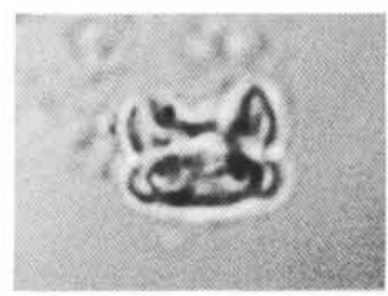

6

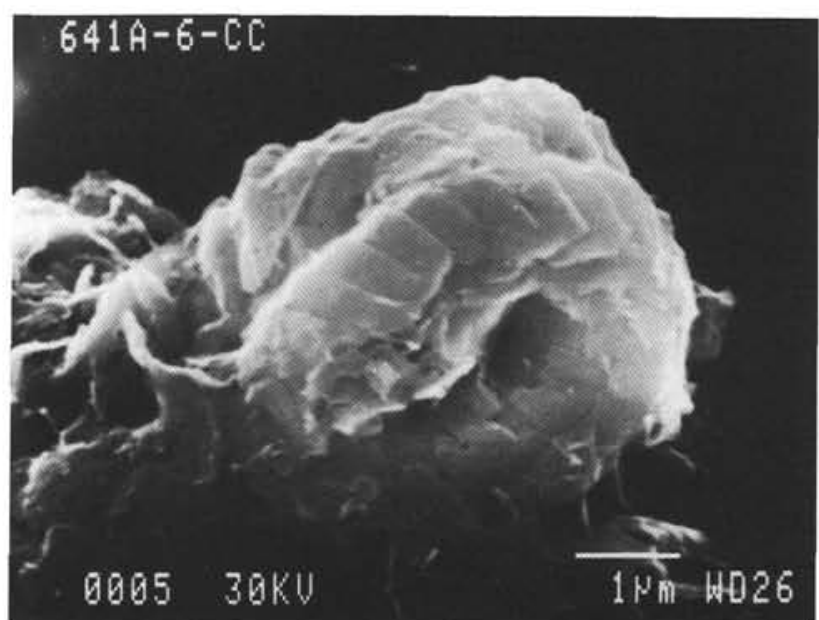

9

Plate 3. All specimens from Sample 103-641A-6X, CC, Cylindralithus nudus Bukry. 1. L, 14,200×. $2^{\text {s1 }}$. Oblique and view, $9500 \times$. $3^{\text {s1 }}$. P, $11,000 \times .4$ and $5^{\text {s1 }} .3300 \times,(4, \mathrm{Tr} ; 5, \mathrm{Pol}) .6$ and $7^{\mathrm{s8}} .3000 \times,(6, \mathrm{Tr} ; 7, \mathrm{Pol}) .8 . \mathrm{L}, 13,800 \times$. $9^{\mathrm{s} 8} .10,700 \times$. 


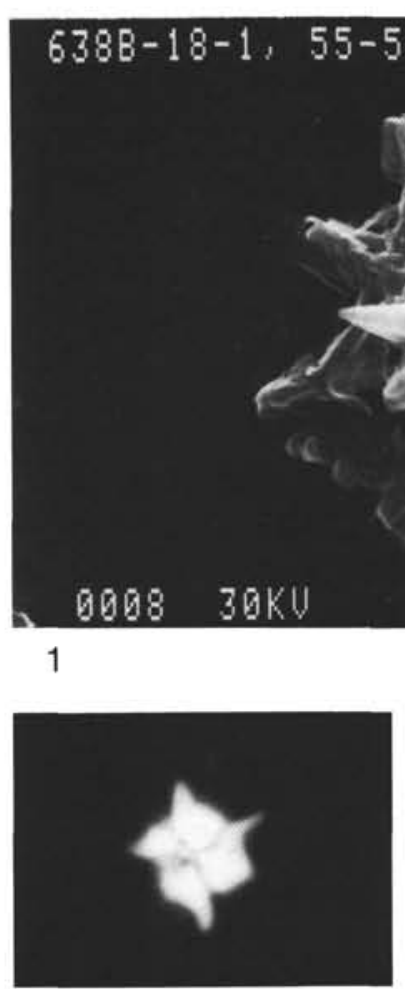

3

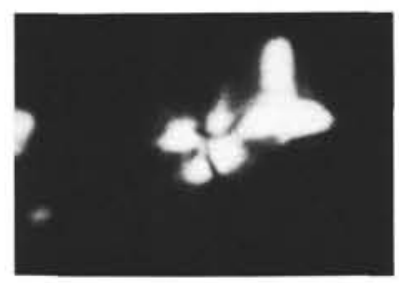

5

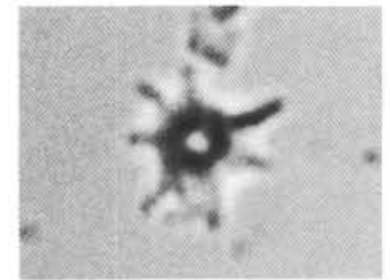

4

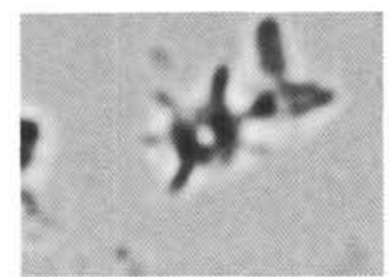

6

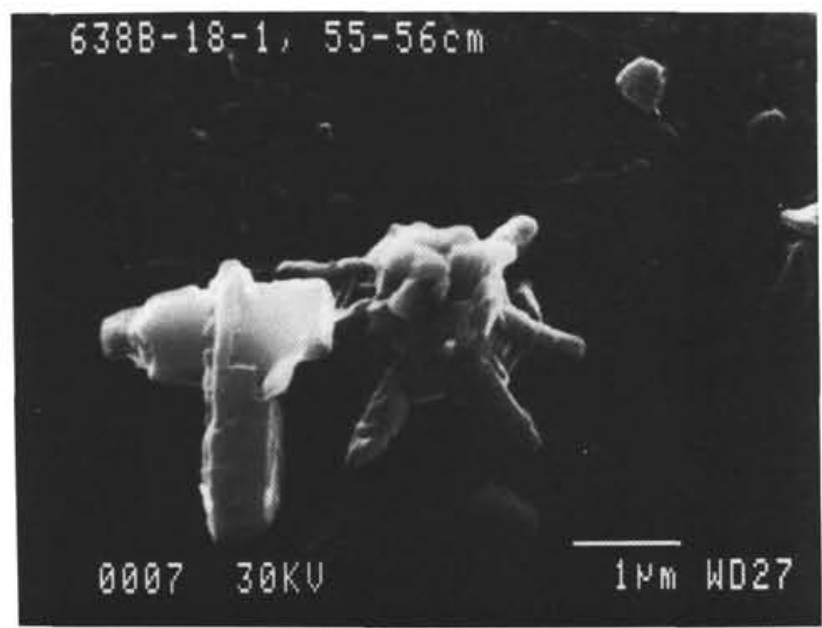

8

Plate 4. 1-8. Hayesites albiensis Manivit, Sample 103-638B-18R-1, 55-56 cm, (1, D, 10,500× $(2-4)^{\mathrm{s}}$, (2, oblique L, 11,000 $\times$; 3, Pol, 4300 $\times$; tr, $4300 \times)$. $(5-8)^{57},(5$, Pol, $3900 \times ; 6, \mathrm{Tr}, 3900 \times ; 7, \mathrm{D}, 11,000 \times ; 8$, oblique L, 11,000 $\times$ ). 9. Eprolithus floralis (Stradner) Sample 103-641A-7X-1, 91-92 cm, oblique L, 10,000×.



2

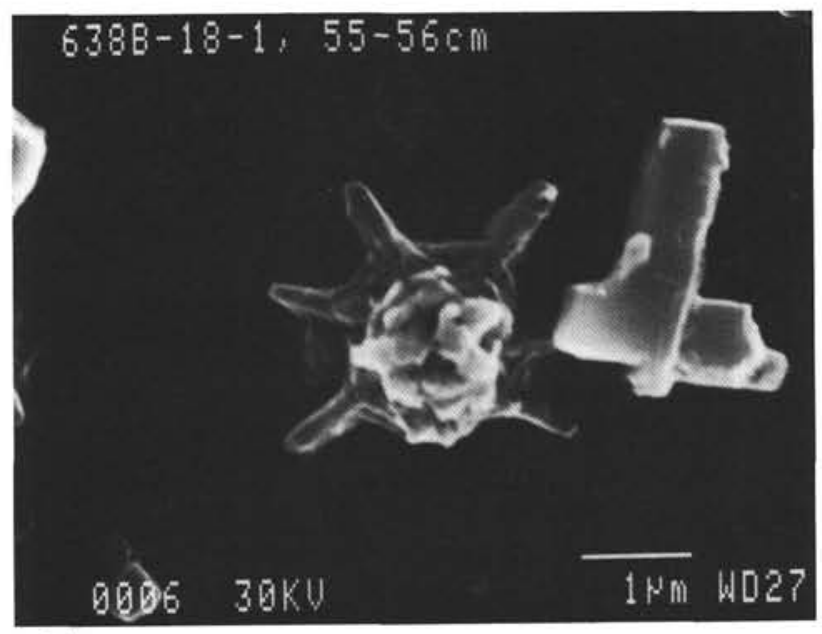

7

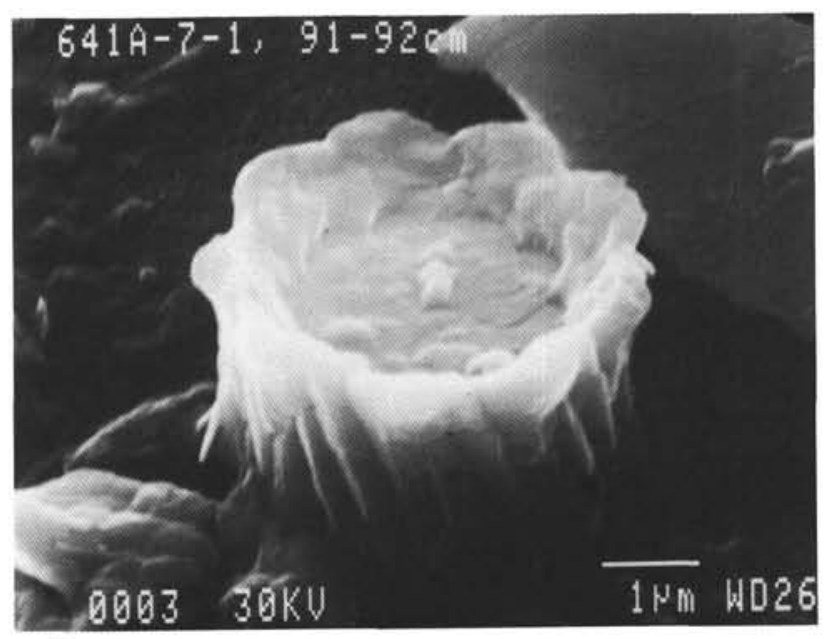

9 


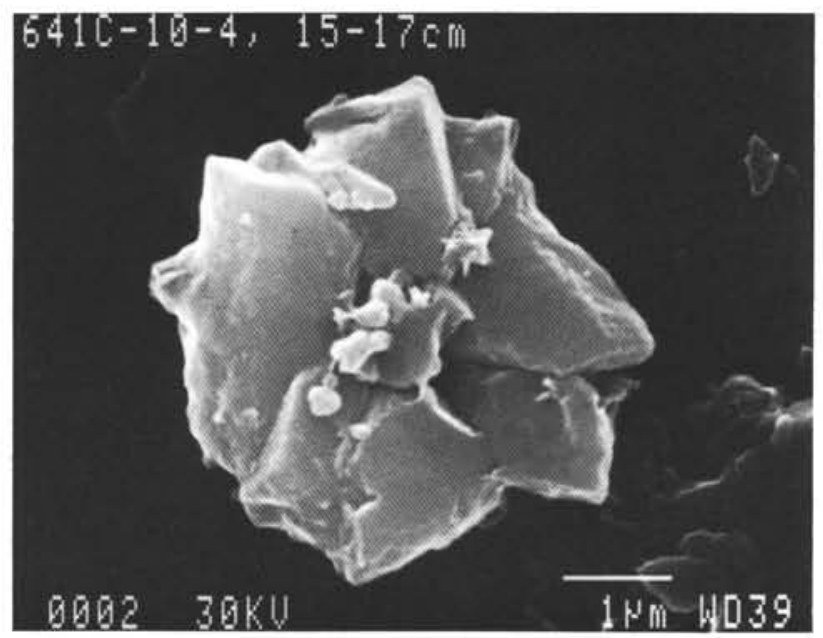

1

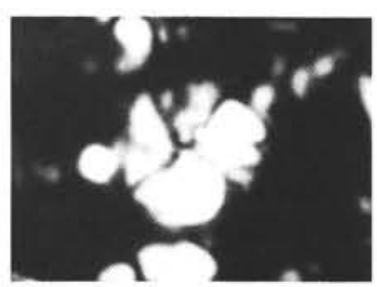

3

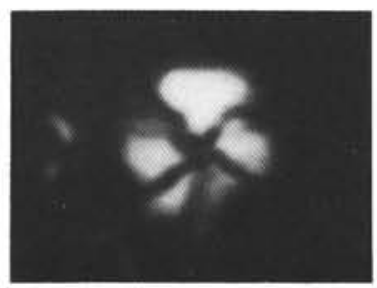

7



4

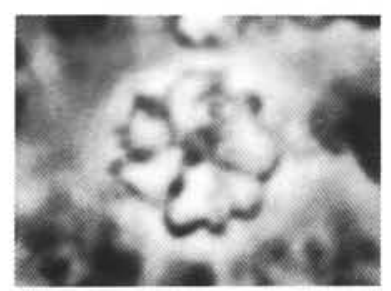

8

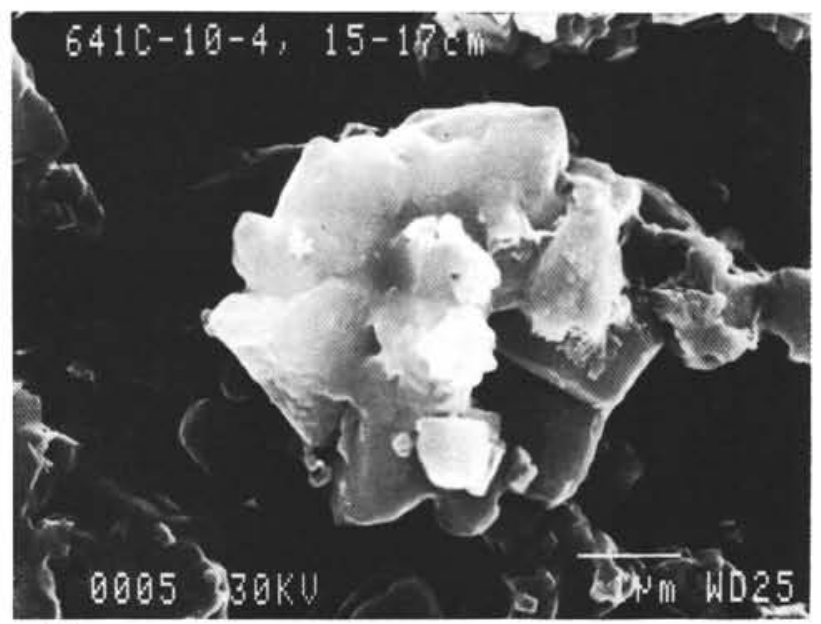

11

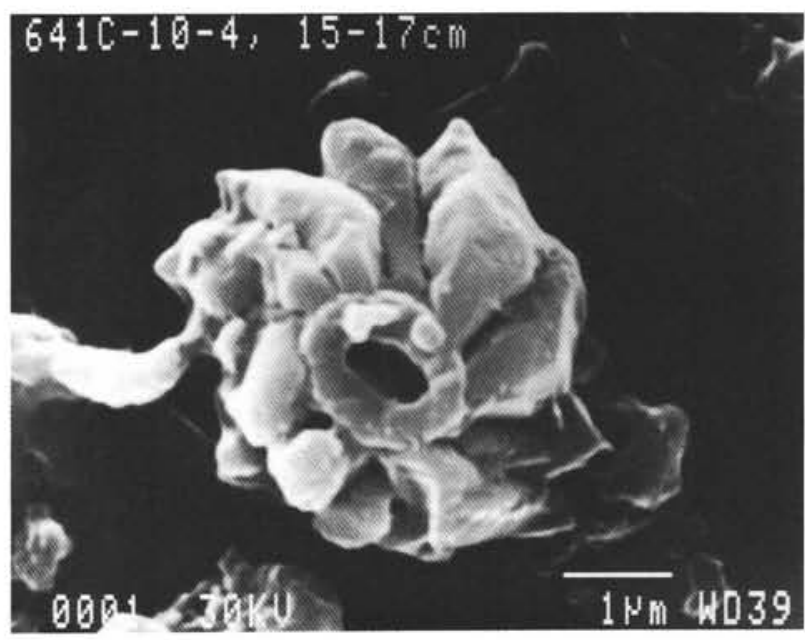

2

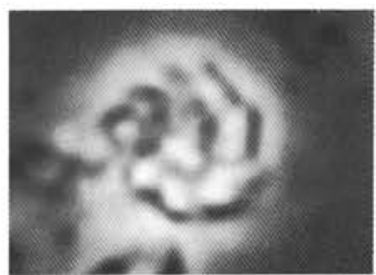

5

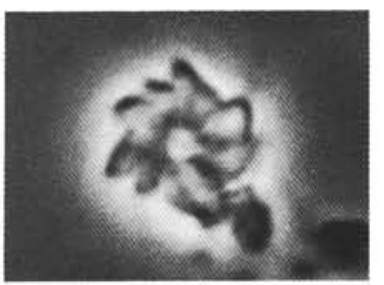

9

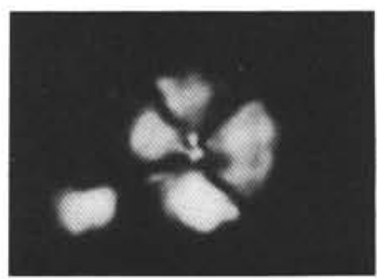

6

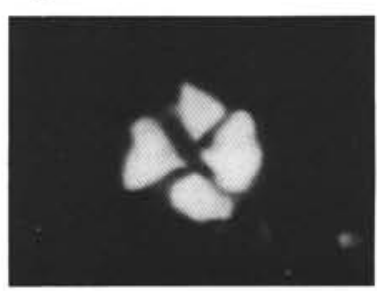

10

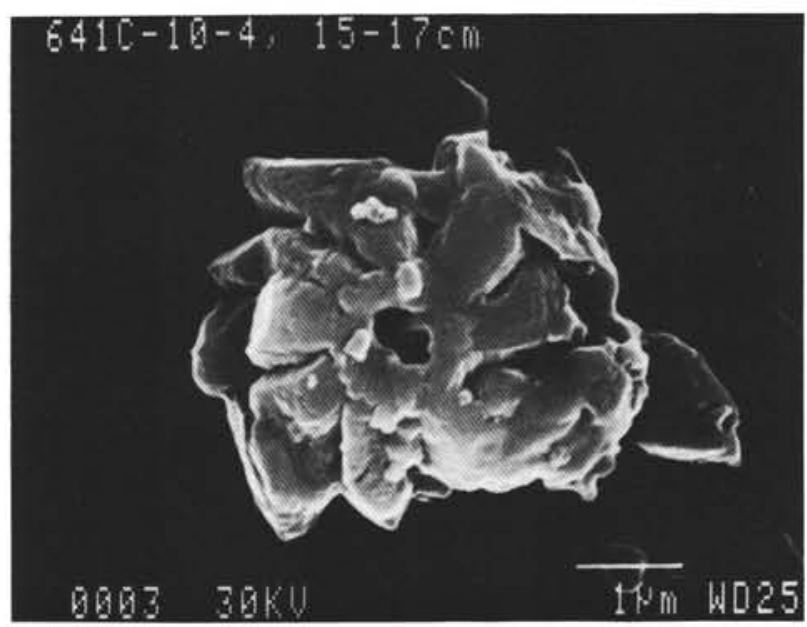

12

Plate 5. All specimens from Sample $103-641 \mathrm{C}-10 \mathrm{R}-4,15-17 \mathrm{~cm}$, Hayesites irregularis (Thierstein). 1. D, 11,000 $\times$. 2. P, 11,000 $\times$. 3 and $4^{\text {s1 }}$. $3900 \times,(3, \mathrm{Pol} ; 4, \mathrm{Ph}) .5$ and $6^{\mathrm{s2}}$. $3500 \times,(5, \mathrm{Pol} ; 6, \mathrm{Ph}) .7$ and $8^{\mathrm{si1}} .3800 \times,(7, \mathrm{Pol} ; 8, \mathrm{Ph}) .9$ and $10^{\mathrm{si2}} .3500 \times,(9, \mathrm{Pol} ; 10, \mathrm{Ph}) . \quad 11 . \mathrm{D}$, $10,500 \times$. 12. P, $10,800 \times$. 


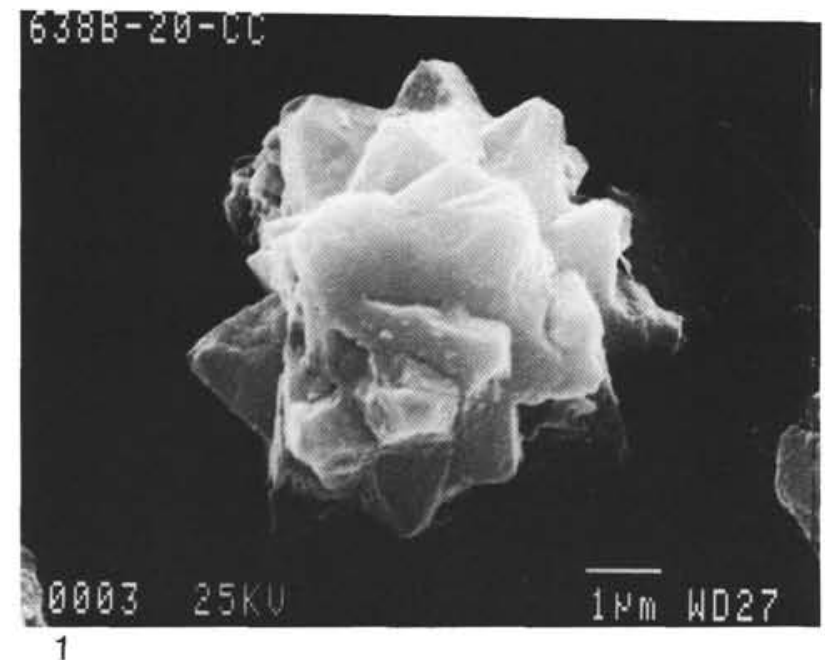

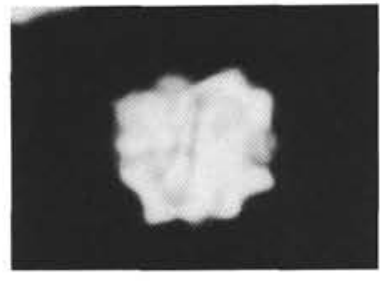

2

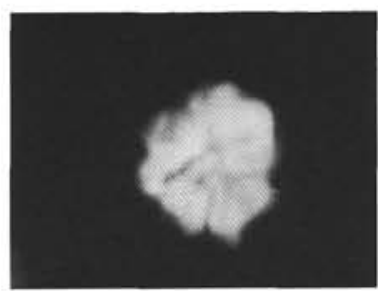

4

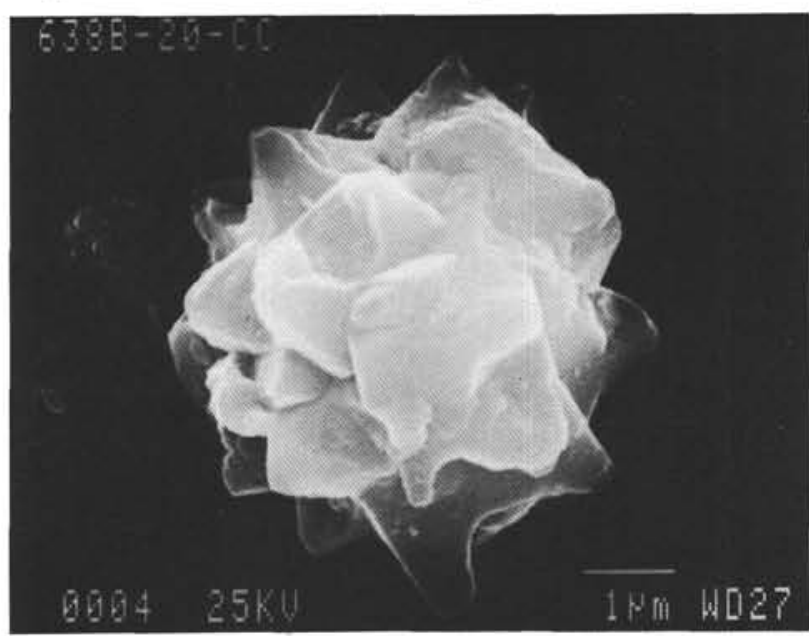

7

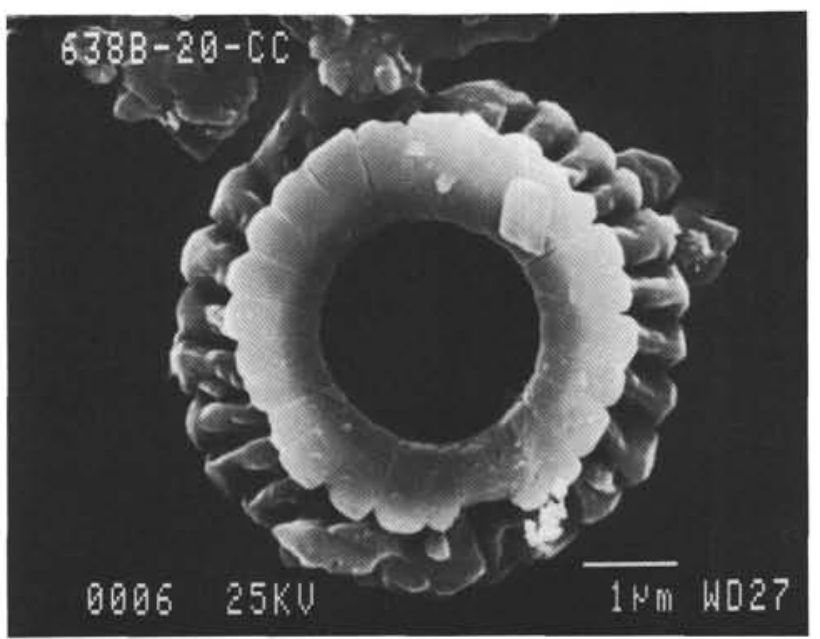

9

Plate 6. All specimens from Sample 103-638B-20R, CC. 1-8. Rucinolithus terebrodentarius Applegate, Covington, and Wise, $\left(1,7500 \times ; 2^{\text {s1 }}\right.$, Pol, $\left.3100 \times ; 3^{51}, \mathrm{Tr}\right),\left(4^{57}\right.$, Pol, $2900 \times ; 5^{57}, \mathrm{Tr} ; 6^{57}, 0^{\circ}$ from "polar axis," $9400 \times ; 7^{56}, 65^{\circ}$ from polar axis, $\left.9300 \times\right)$, $(8,8000 \times$. 9. Diazomatolithus lehmanii Noel, D, $9500 \times$. 


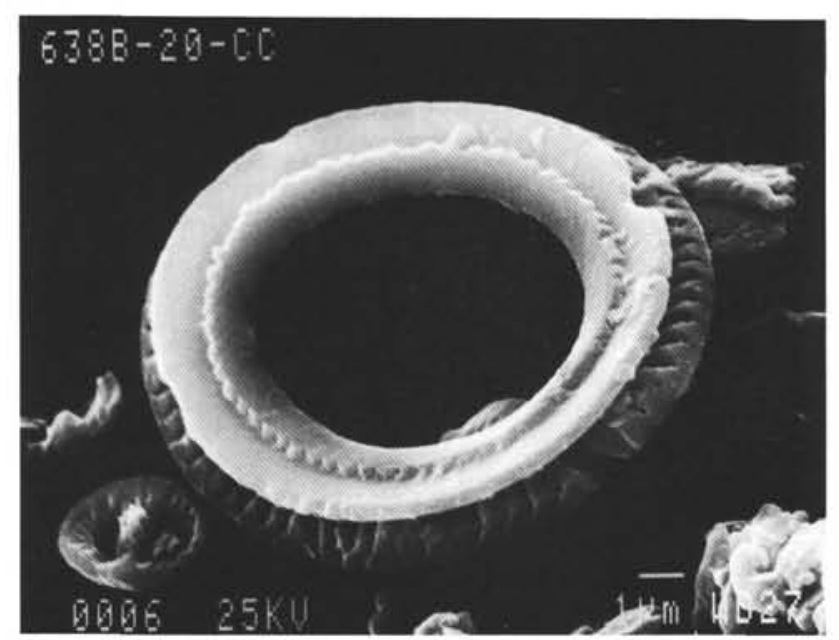

1



6

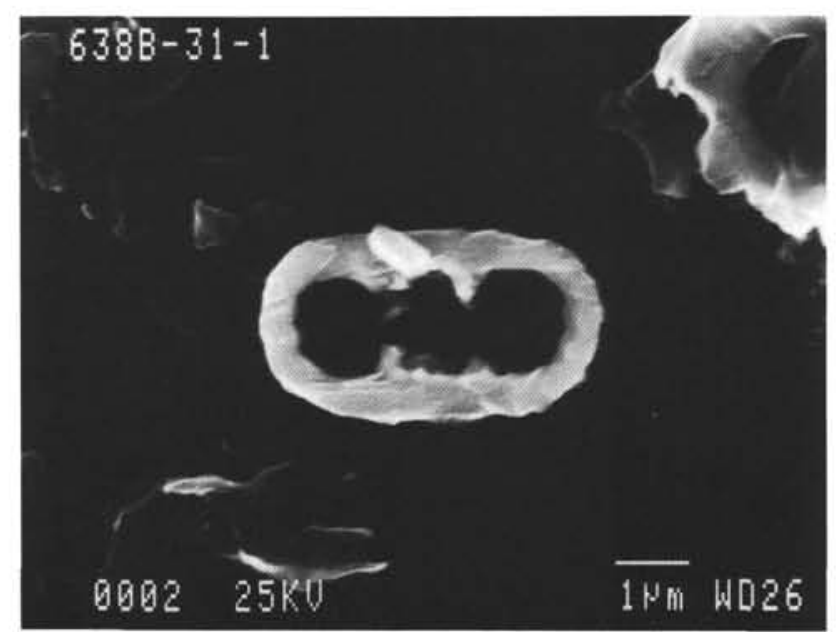

11



2

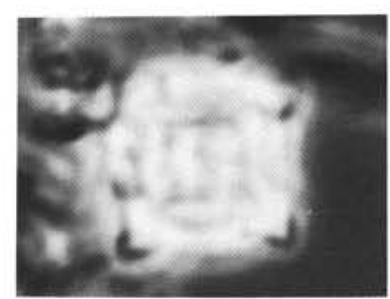

4

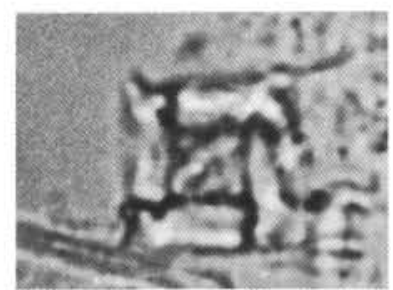

7

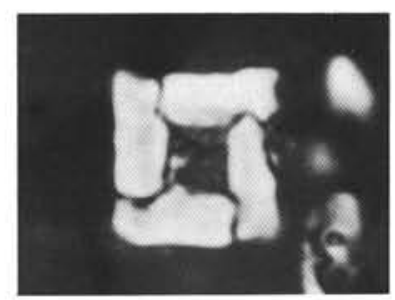

9

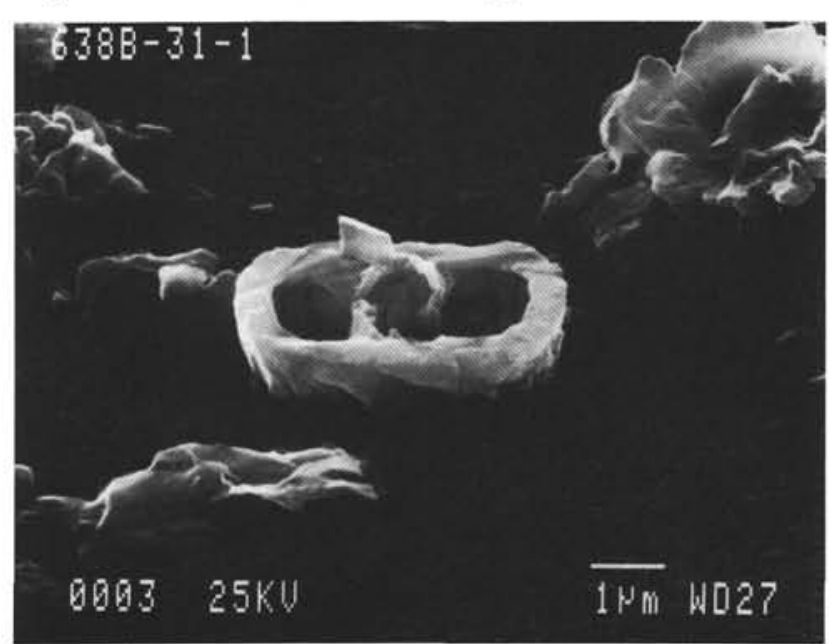

12

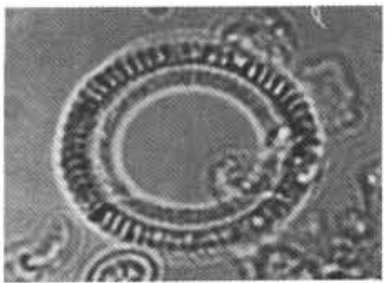

3

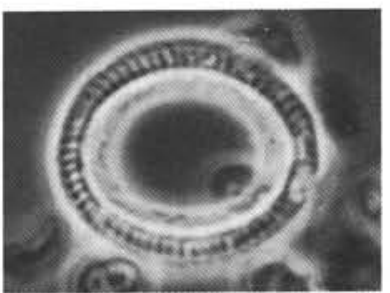

5

8

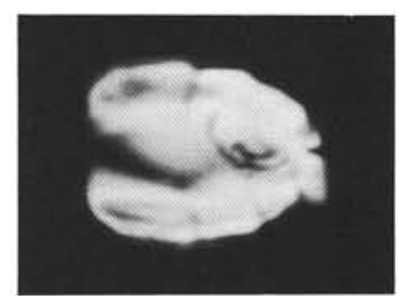

10

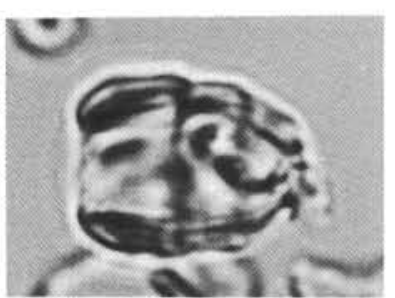
Plate 7. 1-3, 5, and 6. Manivitella pemmatoidea (Deflandre), Sample 103-638B-20R, CC, (1, D, 4700 $\left.\times ; 2^{\mathrm{s1}}, \mathrm{pol}, 1760 \times ; 3^{\mathrm{s1}}, \mathrm{Tr} ; 5^{\mathrm{s1}}, \mathrm{Ph}\right),(6), \mathrm{Sam}-$
ple 103-638B-20R-3, 32-33 cm, D, $8000 \times$. 4, 7, and 8. cf. Dictyolithus quadratus (Gorka), Sample 103-638B-22R-3, 55-56 cm, 2900 $\times$, (4, Ph: 7, Tr; 8, Pol). 10. Lapodeacassis sp. Sample 103-638B-20R, CC, $2300 \times(9, \mathrm{Tr} ; 10, \mathrm{Pol}) .11$ and 12. Zeugrhabdotus pseudoangustus Bralower, Applegate, Covington, and Wise, Sample 103-638B-31R-1, 125-126 cm, 7800 $\times(11, \mathrm{D} ; 12$, oblique D). 


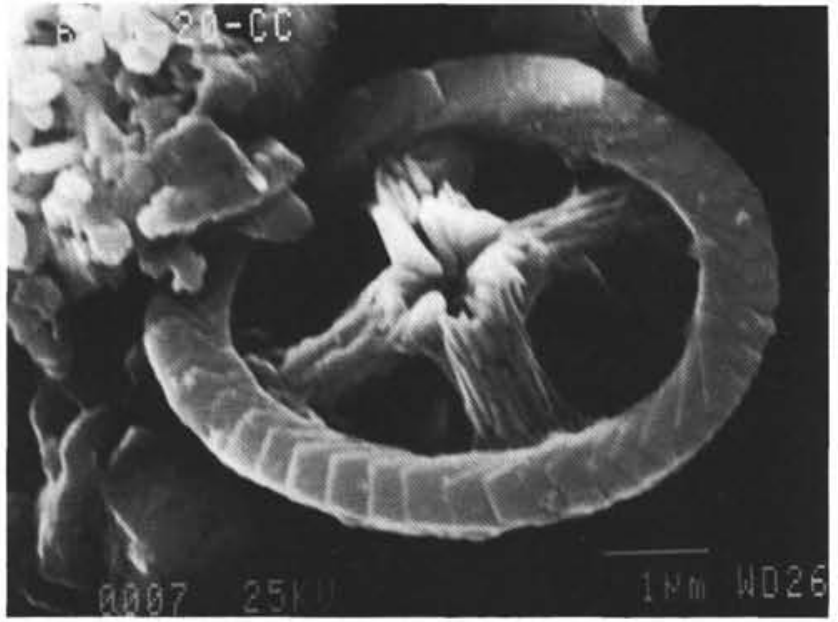

1

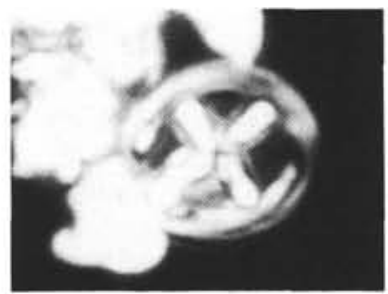

3



5

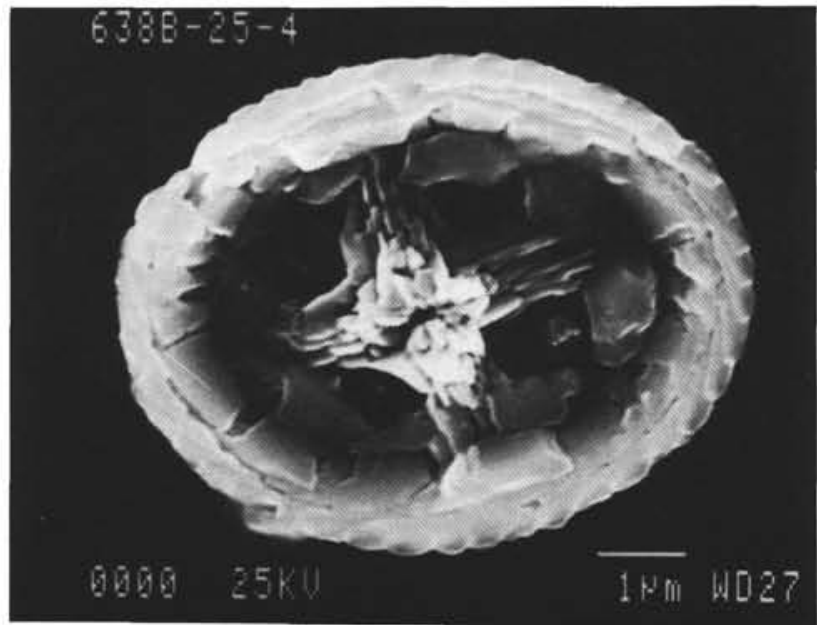

8

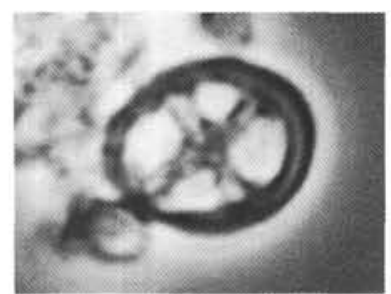

4

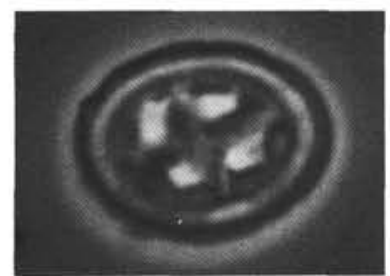

6

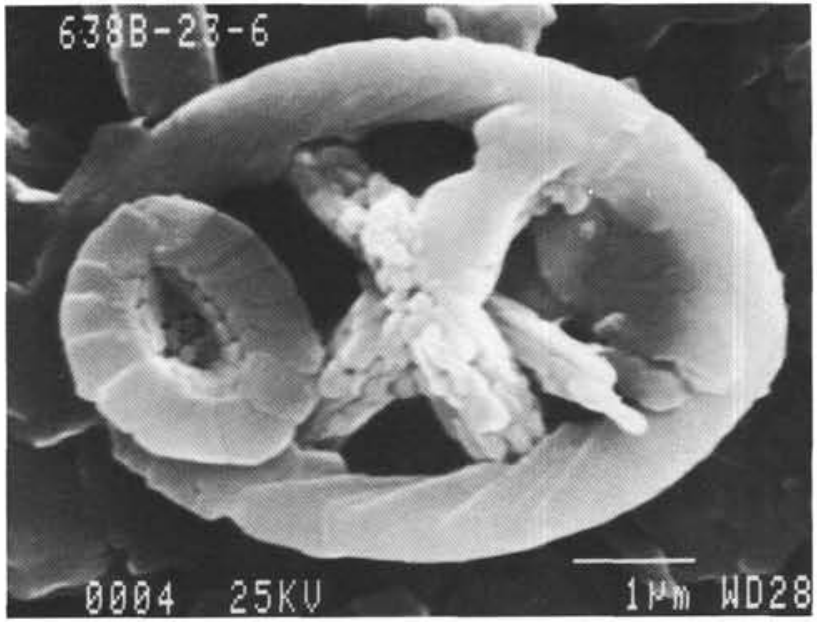

2

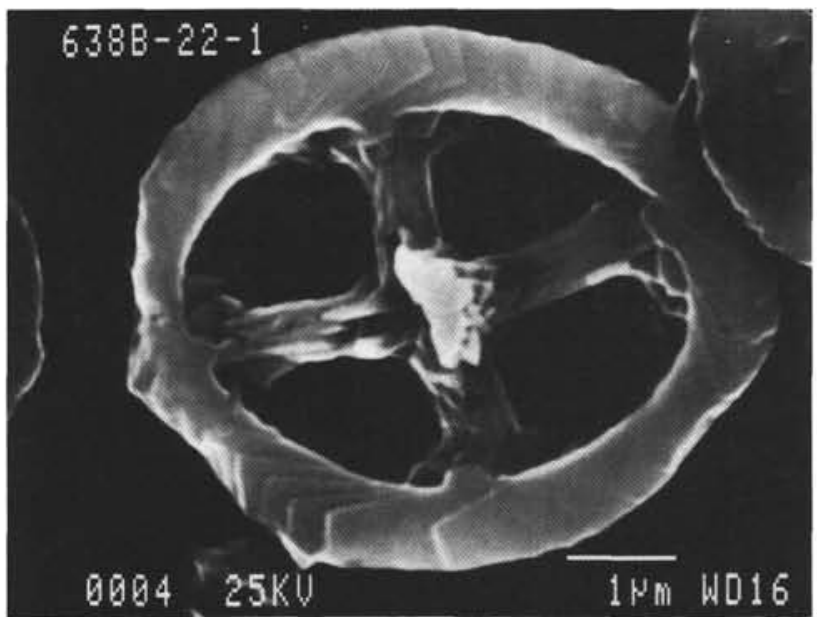

7

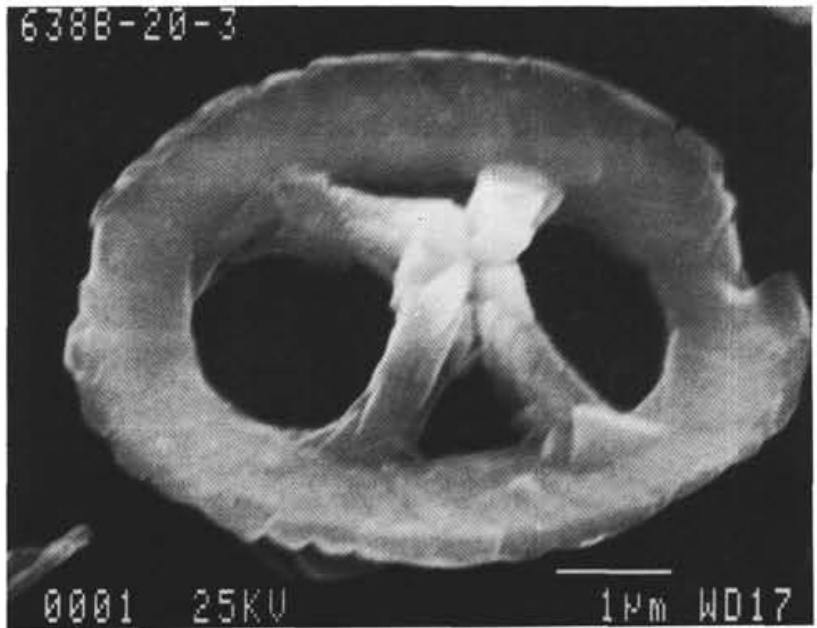

9

Plate 8. 1-4. Chiastozygus litterarius (Gorka). (1) Sample 103-638B-20R, CC, D, 11,000 $\times$, (2) Sample 103-638B-23R-6, 58-59 cm, D, 12,400 $\times$, (3 and 4) ${ }^{\mathrm{si}}, 3700 \times(3, \mathrm{Pol} ; 4, \mathrm{Ph})$. 5, 6, and 8. Vekshinella angusta (Stover), Sample 103-638B-25R-4, $48-49 \mathrm{~cm},(5 \text { and } 6)^{58}, 3600 \times(5, \mathrm{Pol} ; 6, \mathrm{Ph}),(8)$ D, $9000 \times$. 7. Chiastozygus sp., Sample 103-638B-22R-1, 41-42 cm, D, 11,100×. 9. Tegumentum stradneri Thierstein, Sample 103-638B-20R-3, $32-33 \mathrm{~cm}, \mathrm{D}, 12,100 \times$. 


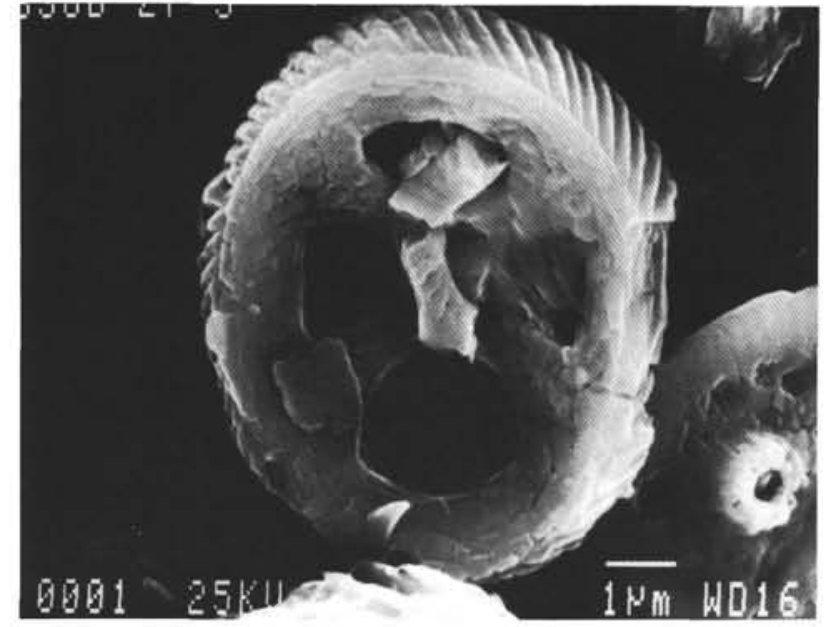

1

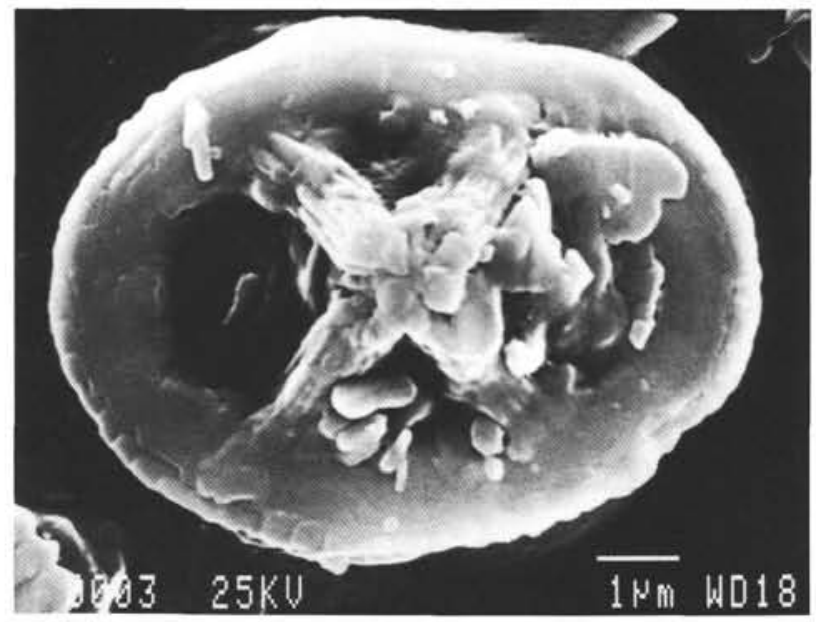

6

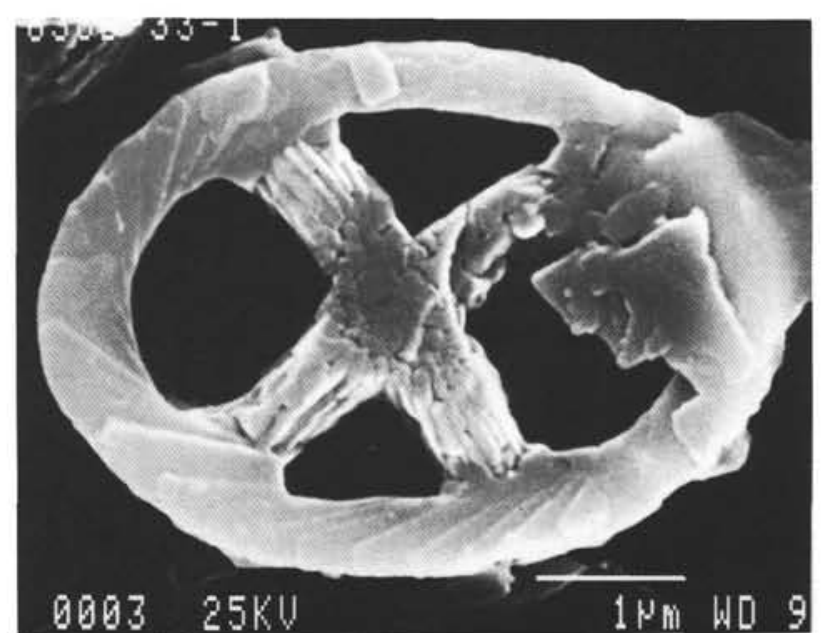

11

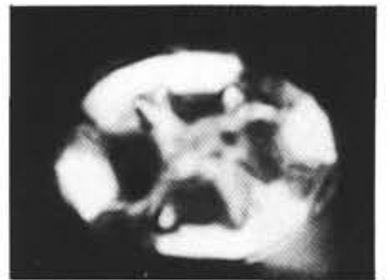

2



4

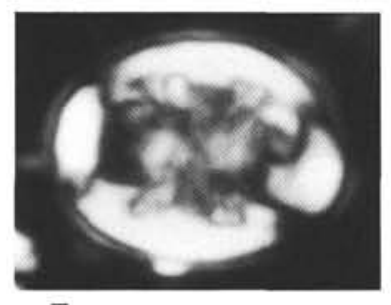

7

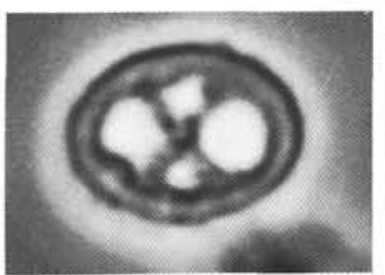

9

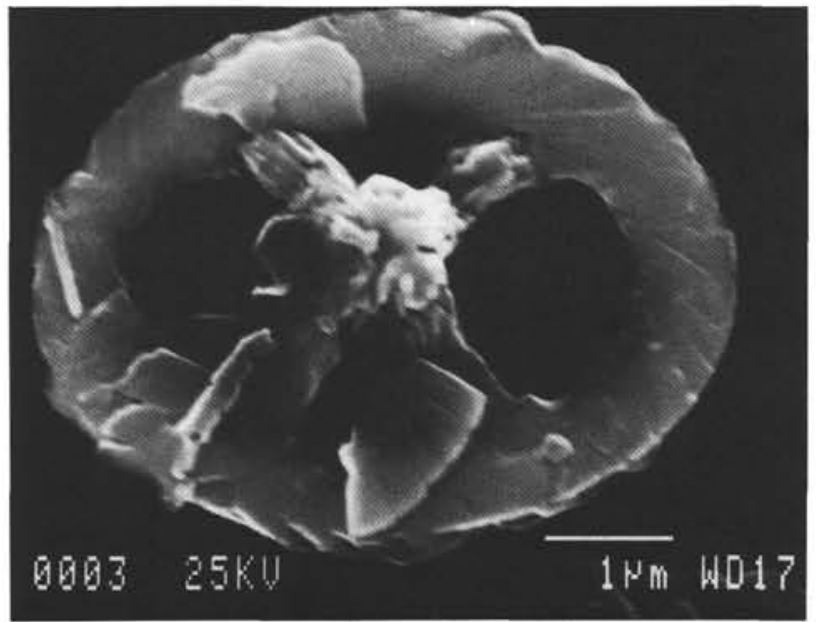

12

Plate 9. 1-7. Eiffellithus striatus (Black), (1-5) Sample 103-638B-27R-5, 54-55 cm, (1) P, 7200 $\times,(2-5)^{51} 3300 \times,\left(2, \mathrm{Pol}, 0^{\circ}\right.$ to CN; $3, \mathrm{Pol}, 10^{\circ}$ to $\mathrm{CN} ; 4$, Pol, $45^{\circ}$ to CN; 5, Tr), (6) D, Sample 103-638B-30R-5, 5-6 cm, $8500 \times$, (7) ${ }^{\mathrm{s} 6}$, Pol, $10^{\circ}$ to CN. $8-10$ and 12. Chiastozygus tenuis Black, Sample 103-638B-27R-5, 54-55 cm, $(8-10)^{\mathrm{s} 12}(8, \mathrm{Tr} ; 9, \mathrm{Ph}, 10$, Pol) (12) D, 13,000 $\times$. 11. Chiastozygus litterarius (Gorka), Sample 103-638B-33R-1, 75$76 \mathrm{~cm}, 15,300 \times$. 

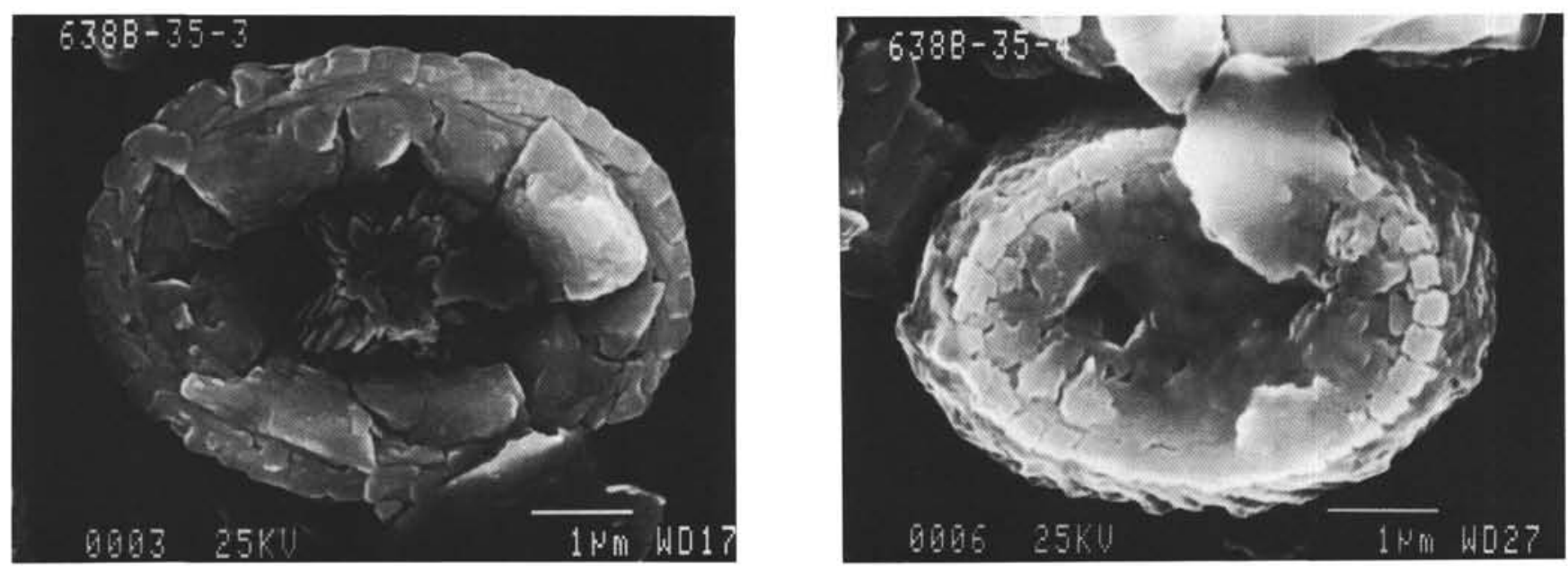

1

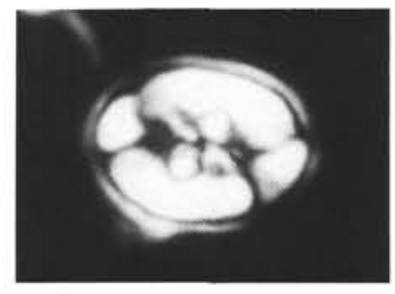

3

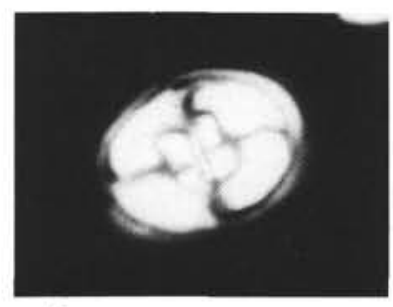

5

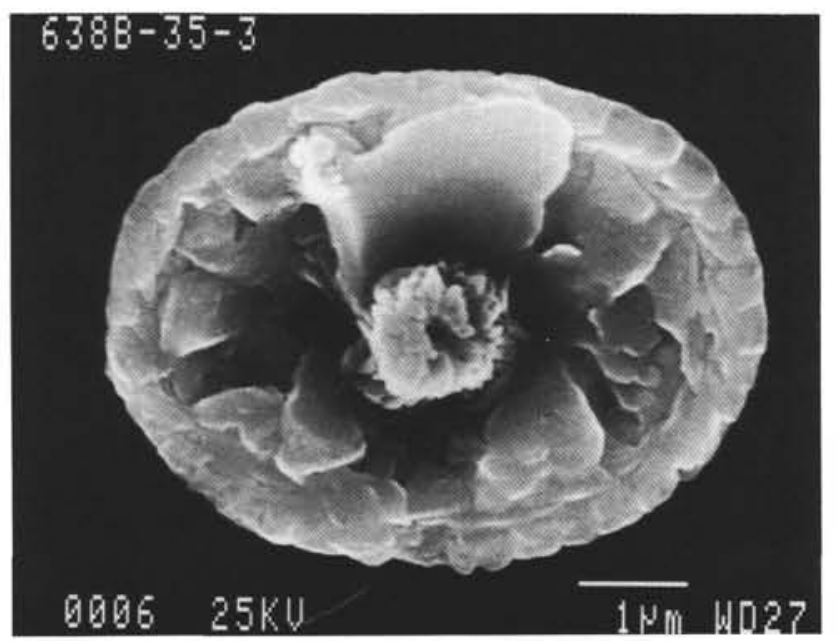

8

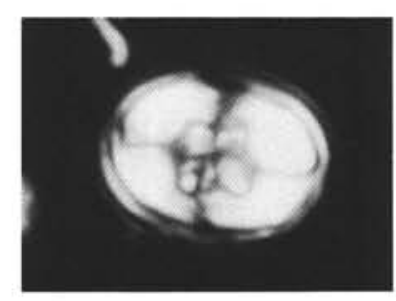

4

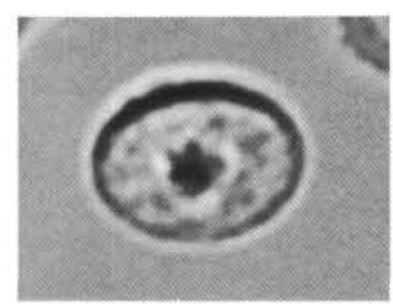

6

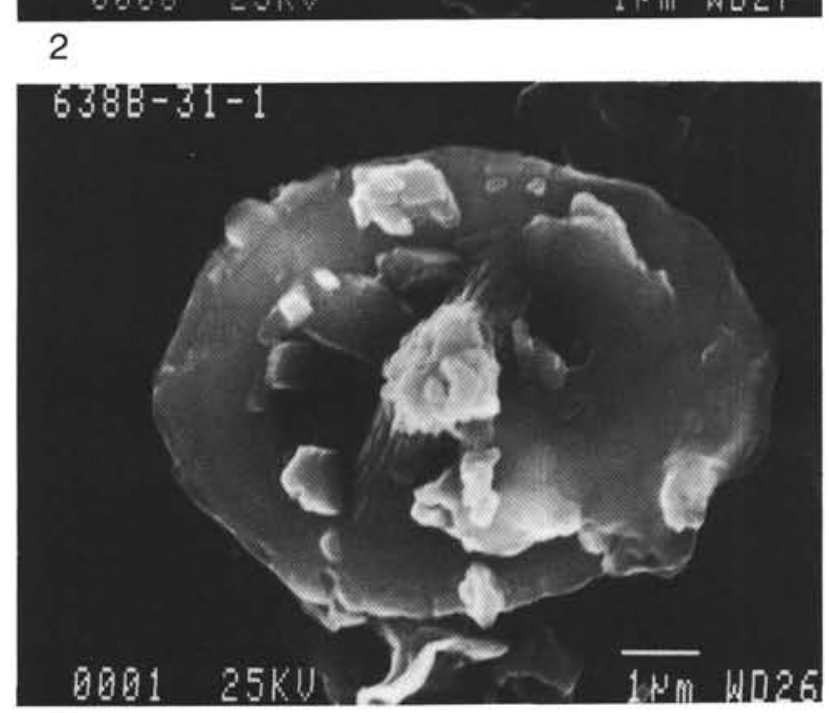
7

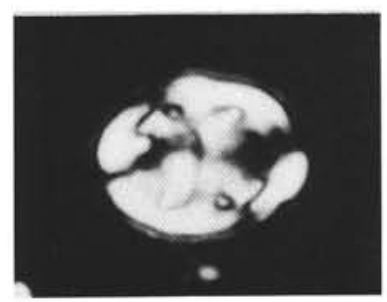

9

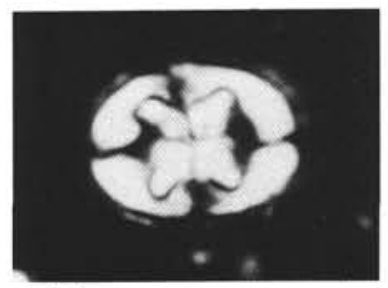

11

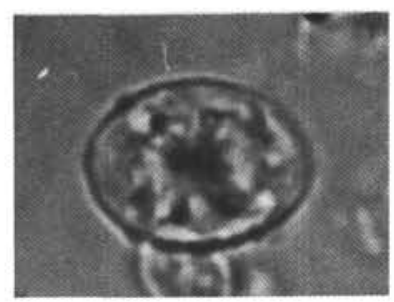

10

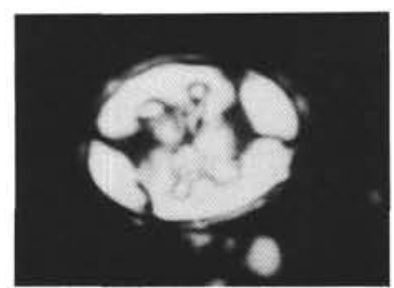

12

Plate 10. 1-6 and 8. Eiffellithus windii Applegate and Bergen n. sp. (1) Sample 103-638B-35R-3, 111-112 cm, D, 11,400×, holotype. (2) Sample $103-638 \mathrm{~B}-35 \mathrm{R}-4,34-35 \mathrm{~cm}, \mathrm{P}, 12,600 \times$. (3 and 4) $)^{\mathrm{si}}, 4900 \times,\left(3, \mathrm{pol}, 0^{\circ}\right.$ to $\mathrm{CN} ; 4, \mathrm{pol}, 10^{\circ}$ to $\left.\mathrm{CN}\right)$. $(5 \text { and } 6)^{\mathrm{s}}, 3500 \times,(5, \mathrm{pol} ; 6, \mathrm{Tr}),(8, \mathrm{D}$, $11,500 \times)$. 7 and 9-12. Eiffellithus striatus (Black), Sample 103-638B-31R-1, 125-126 cm, (7) D, 7800 × , (9-12) ${ }^{57}, 2700 \times .\left(9, \mathrm{pol}, 0^{\circ}\right.$ to CN; 10, Tr; 11 , pol, $45^{\circ}$ to $\mathrm{CN} ; 12$, pol, $10^{\circ}$ to $\mathrm{CN}$ ). 


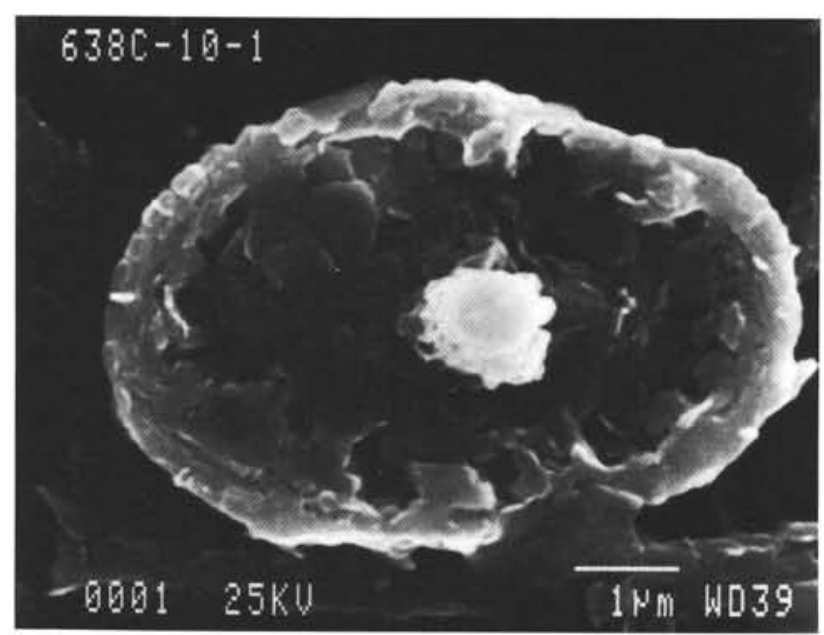

1

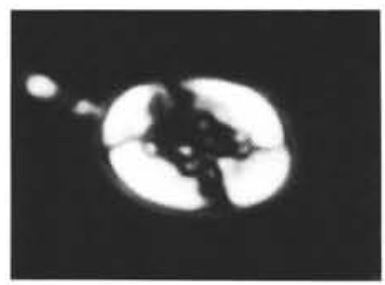

6

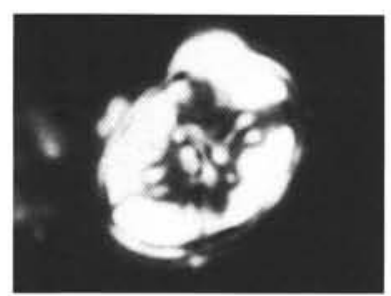

10

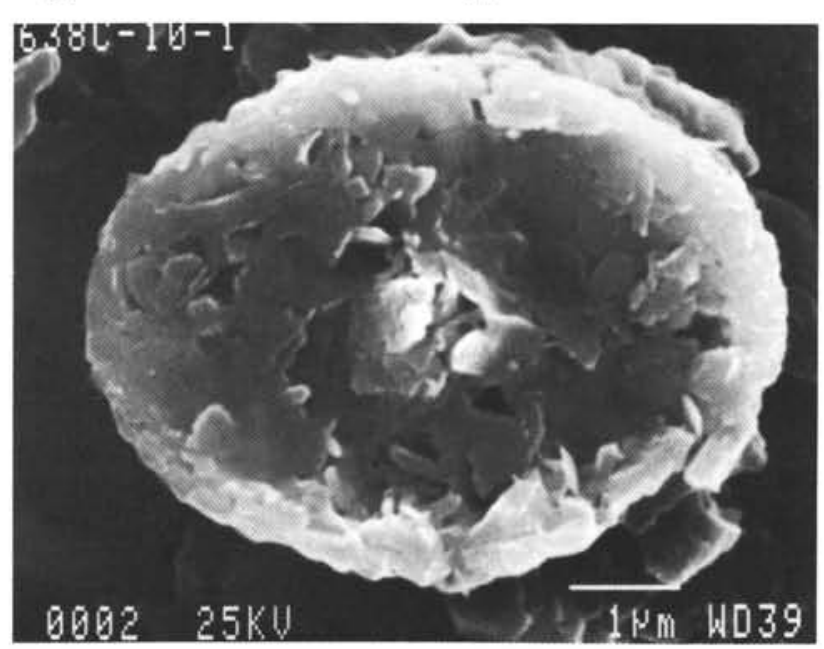

14
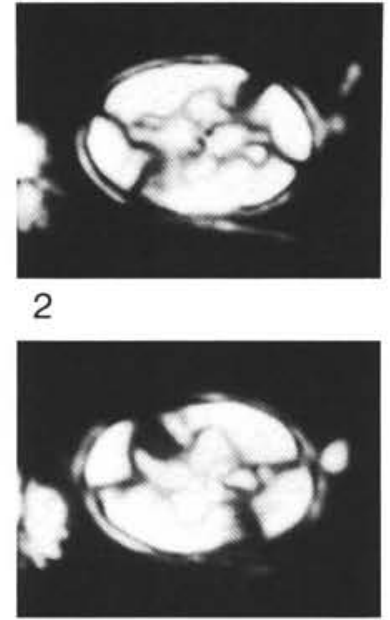

4

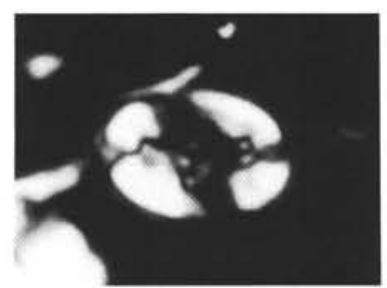

8

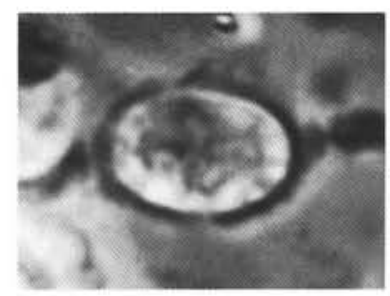

12

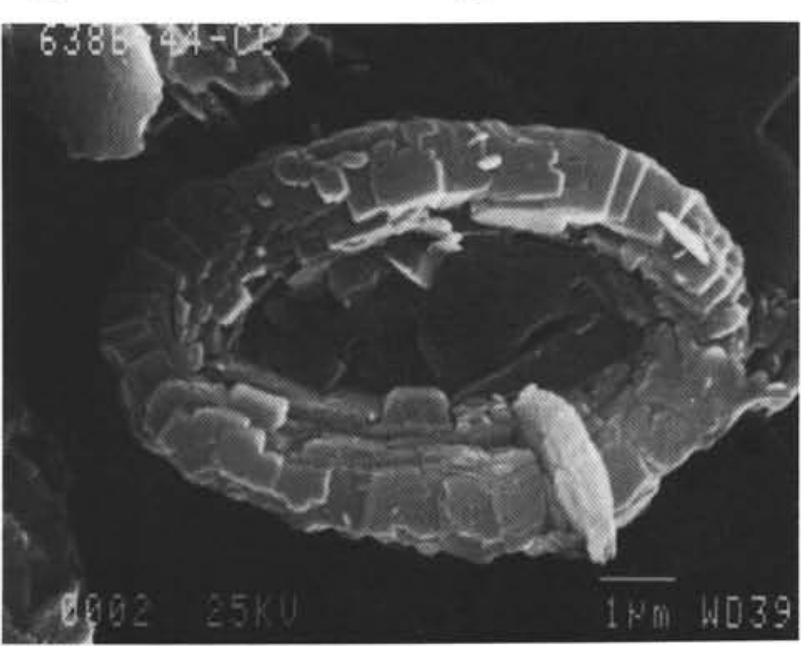

15

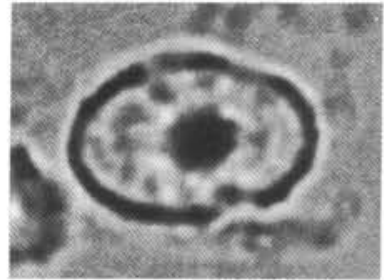

3

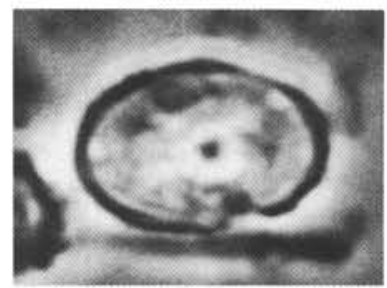

5

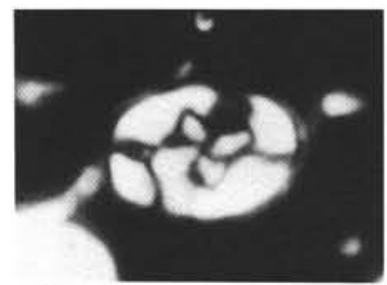

9

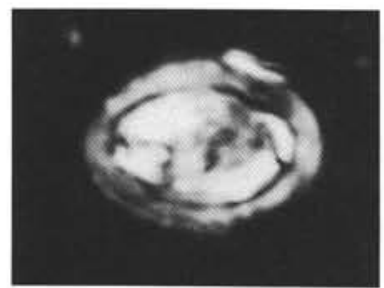

13

Plate 11. 1-12 and 14. Eiffellithus primus Applegate and Bergen n. sp., Sample 103-638C-10R-1, 35-36 cm, (1) D, 10,500 $\times$, holotype, (2-5) ${ }^{\mathrm{s} 1}$ $3700 \times,\left(2\right.$, pol, $45^{\circ}$ to $\mathrm{CN} ; 3$, Tr; 4 , pol, $0^{\circ}$ to $\left.\mathrm{CN} ; 5, \mathrm{Ph}\right)$. (6, pol, $45^{\circ}$ to $\mathrm{CN} ; 7$, pol, $0^{\circ}$ to $\left.\mathrm{CN}, 3700 \times\right)$. $(8,9,12) 3700 \times,\left(8, \mathrm{pol}, 45^{\circ}\right.$ to $\mathrm{CN}$; $9^{\mathrm{s}}$, pol, $0^{\circ}$ to $\left.\mathrm{CN}, 12, \mathrm{Ph}\right)$. $(10 \text { and } 11)^{\mathrm{s}} 4 \mathrm{4} 300 \times,\left(10\right.$, pol, $45^{\circ}$ to $\mathrm{CN} ; 11$, pol, $0^{\circ}$ to $\left.\mathrm{CN}\right) .(14, \mathrm{D}, 11,600 \times)$. 13 and 15 . Percivalia cf. $P$. fenestrata (Worsley) (13) ${ }^{\mathrm{s} 15}$ pol, $2900 \times$, (15) D, 7600 $\times$. 


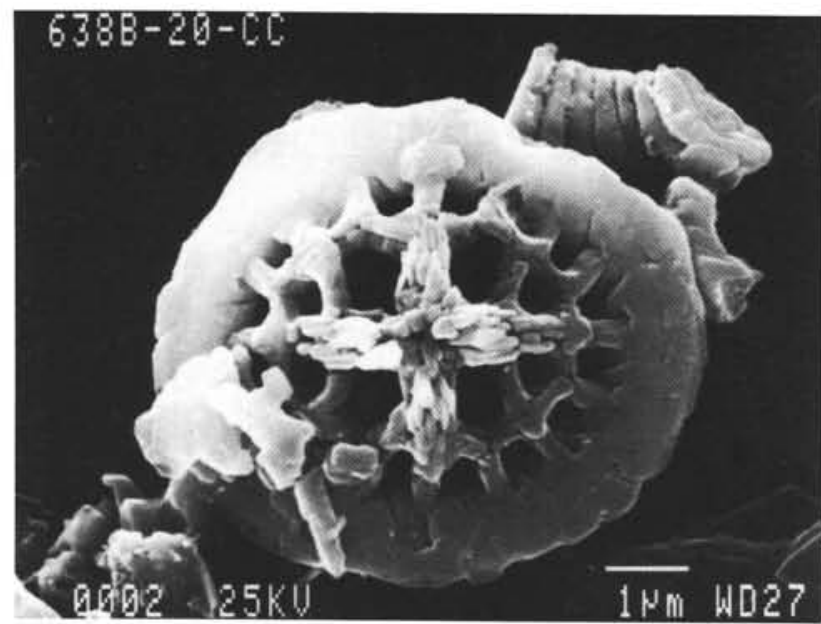

1

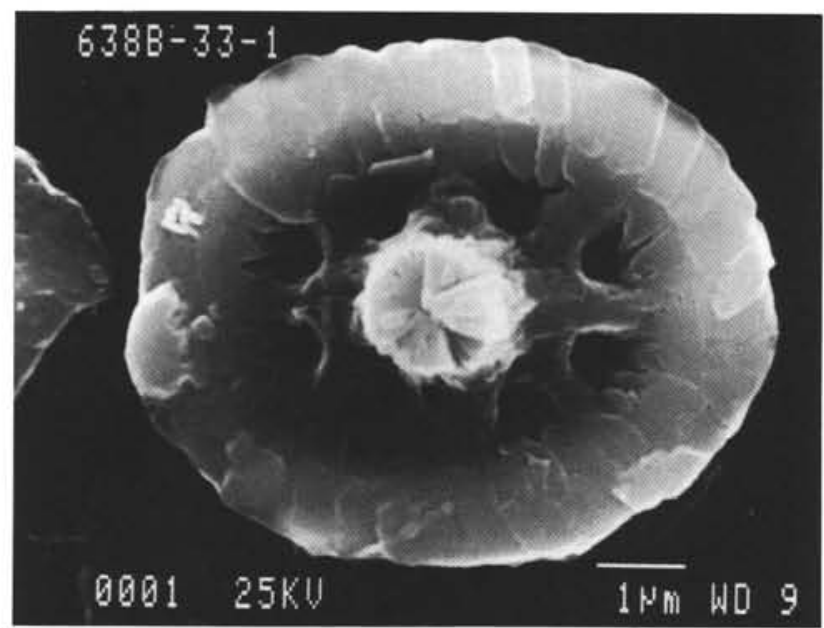

3

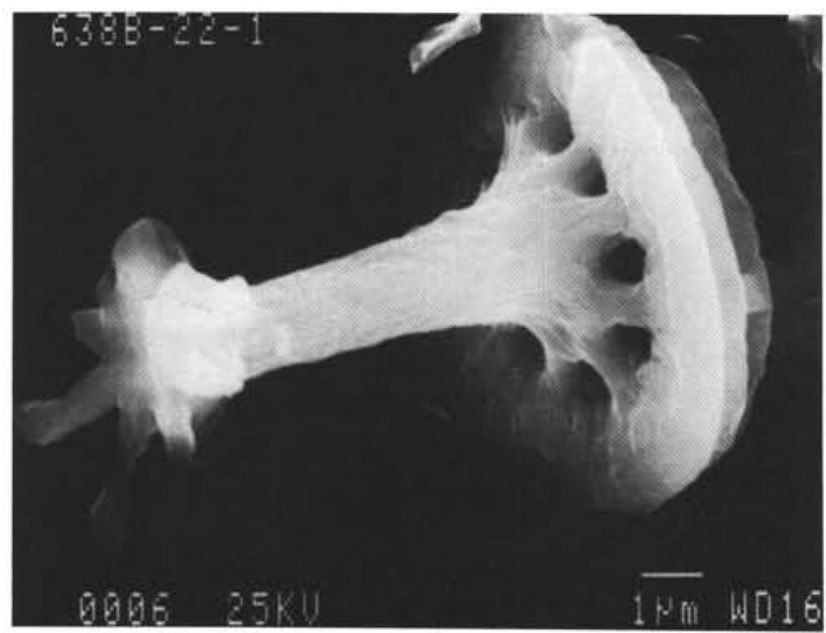

5

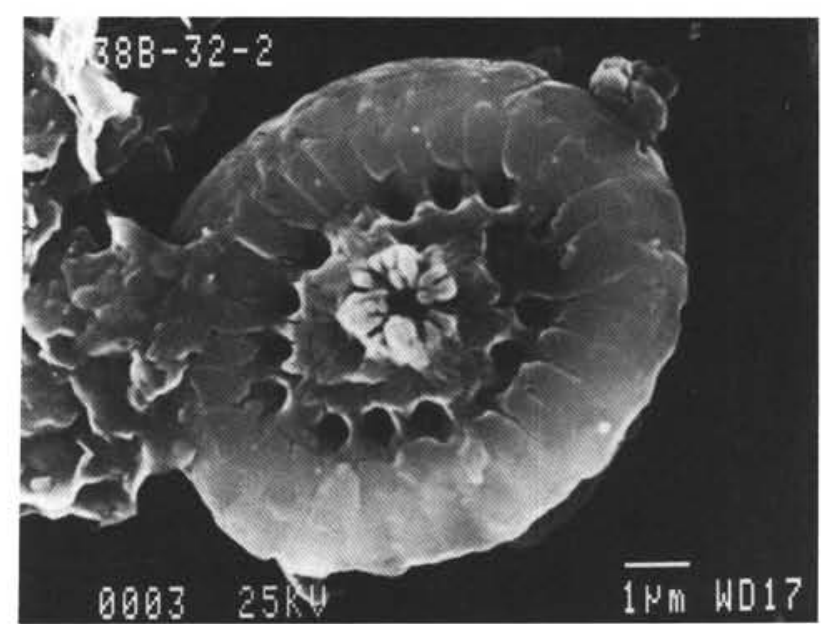

2

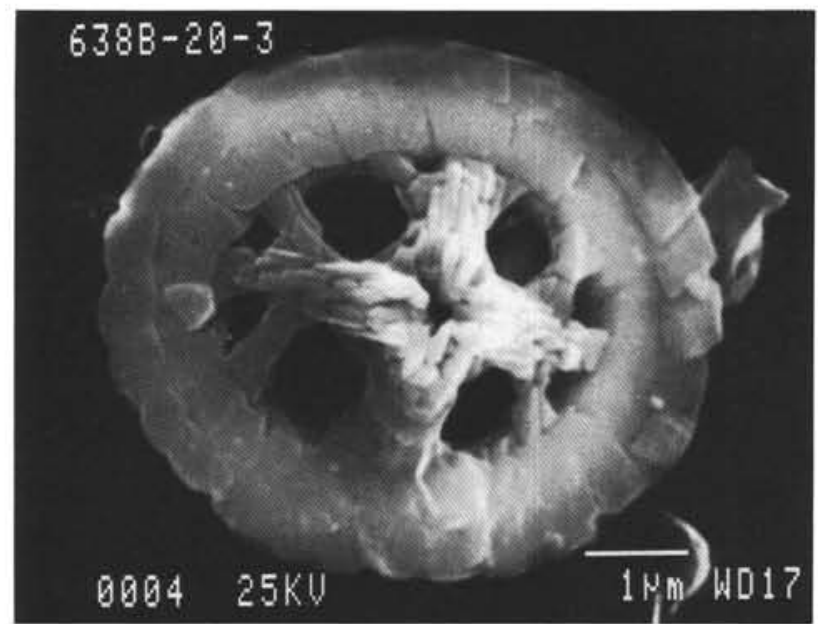

4

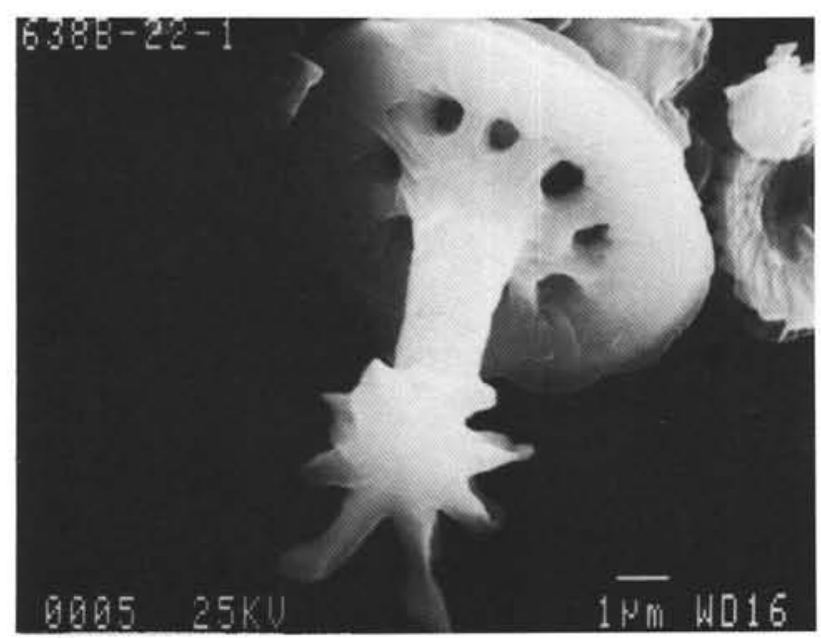

6

Plate 12. 1. Cretarhabdus conicus Bramlette and Martini, Sample 103-638B-20R, CC, D, $8400 \times$. 2. Cretarhabdus surirellus Thierstein, Sample 103-638B-32R-2, 126-127 cm, D, 6700×. 3 and 4. Retecapsa angustiforata Black (3) Sample 103-638B-33R-1, 75-76 cm, D, 9000 $\times$, (4) Sample 103-638B-20R, CC, D, 10,600×. 5 and 6. Retecapsa angustiforata Black and Retecapsa radiata (Worsley), Sample 103-638B-22R-1, 41-42 cm, (5) D, $6100 \times(6) \mathrm{D}, 5200 \times$. 


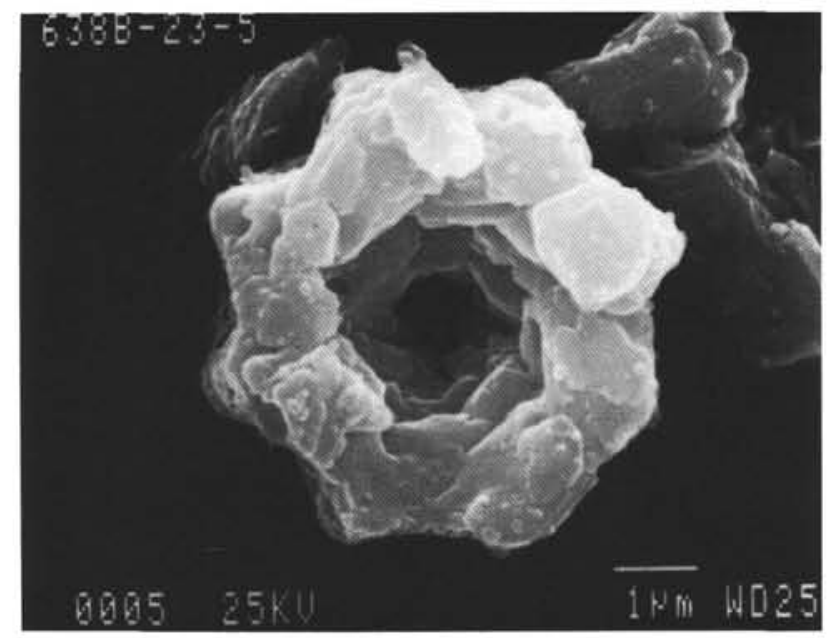

1

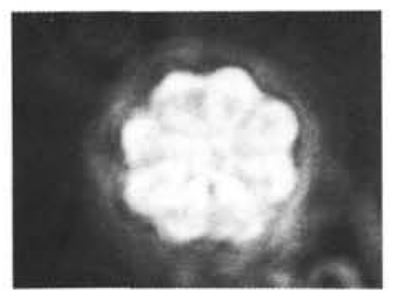

3

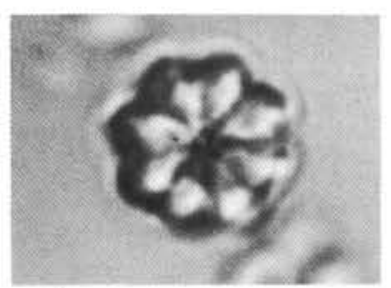

7

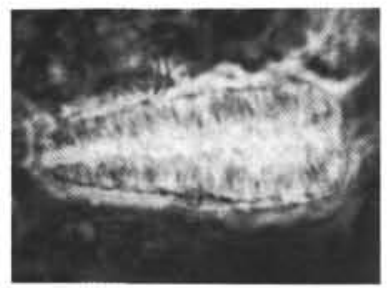

11



13



4

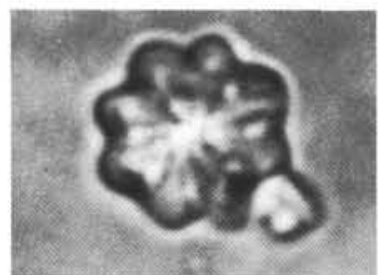

8

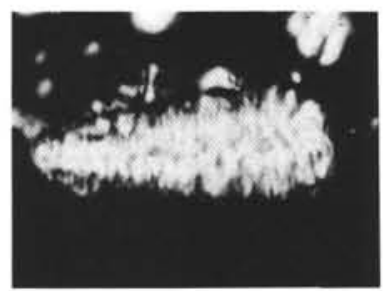

12

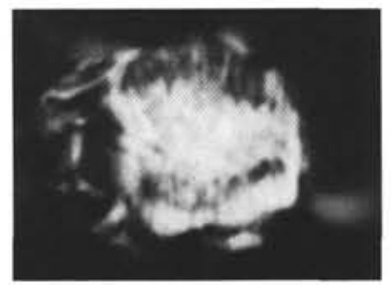

14

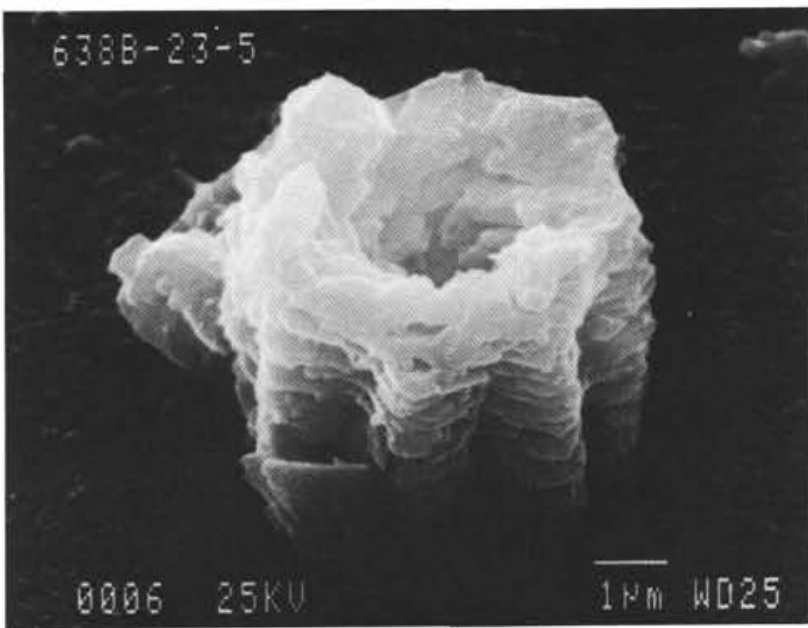

2

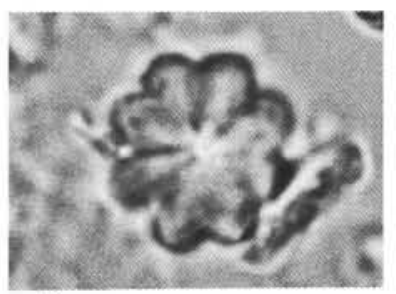

5

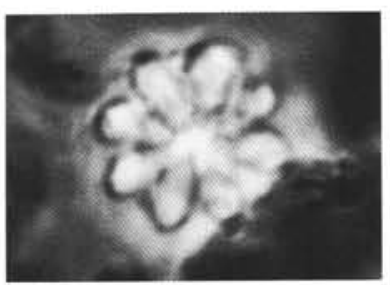

9

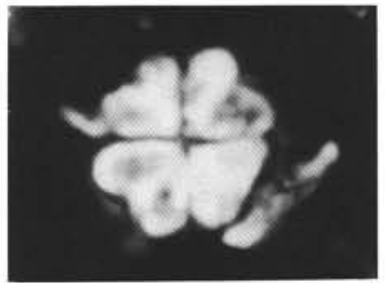

6

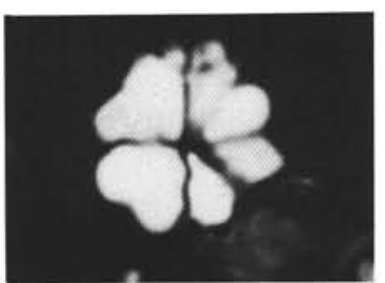

10

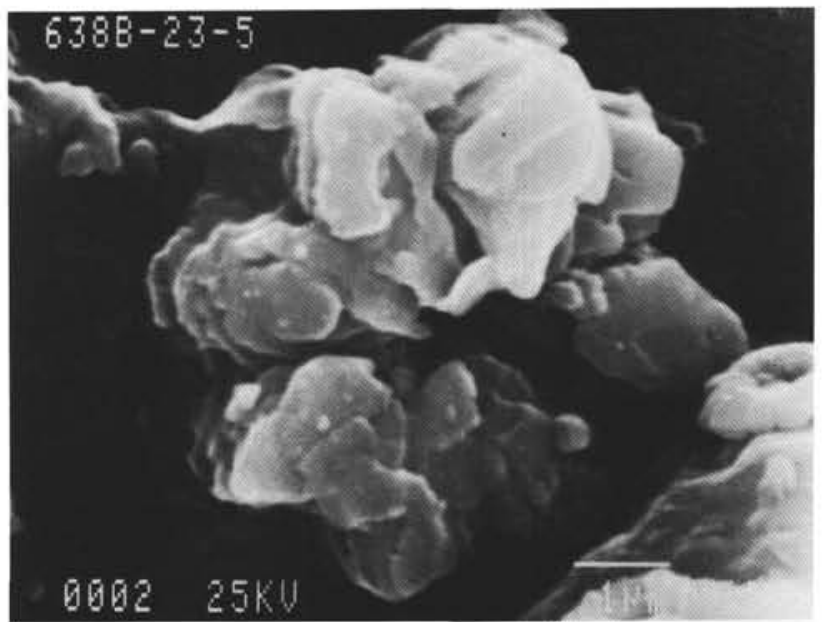

15

Plate 13. 1-10 and 15. Nannoconus ligius Applegate and Bergen n. sp., Sample 103-638B-23R-5, 89-90 cm. (1) $8400 \times$, holotype, (2) $7500 \times$. (38) $3300 \times,\left(3\right.$, pol; 4, Tr; 5, Tr; 6, Pol; 7, Tr; 8, Tr). $\left(9\right.$ and 10) ${ }^{s 15} 3300 \times,(9, \mathrm{Ph} ; \mathrm{Pol}),(15) 10,000 \times$. 11 and 12. Nannoconus bermudezi Bronniman, Sample 103-638B-35R-3, 111-112 cm, 1600,$(11, \mathrm{Ph} ; 12, \mathrm{Pol}) .13$ and 14. Nannoconus bucheri Bronnimann, Sample 103-638B-32R-1, 43-44 cm, $2200 \times,(13, \mathrm{Ph} ; 14, \mathrm{Pol})$. 


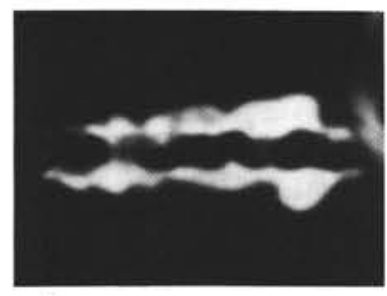

1

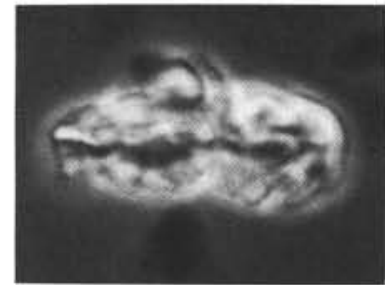

3

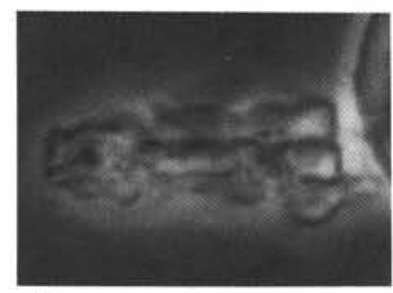

2

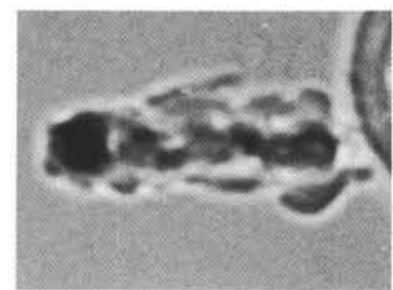

4



6

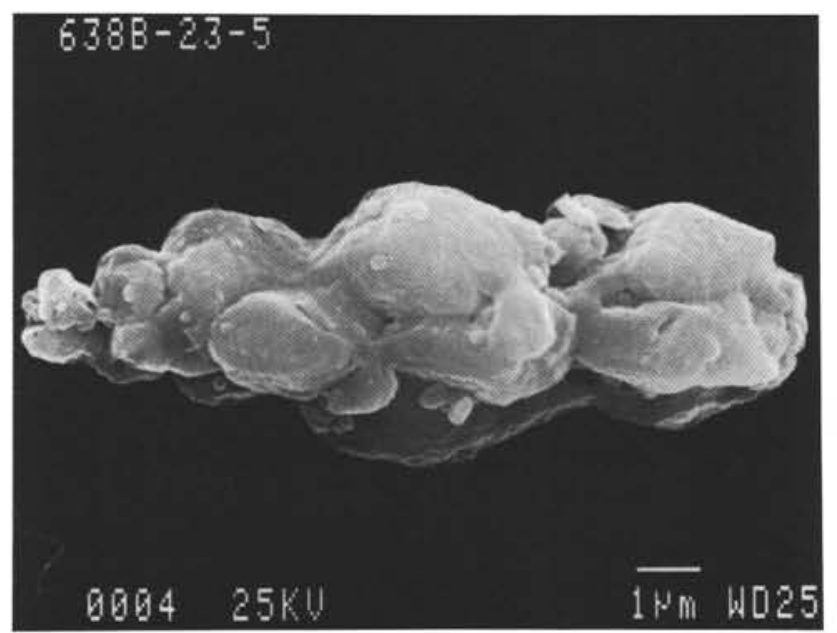

8

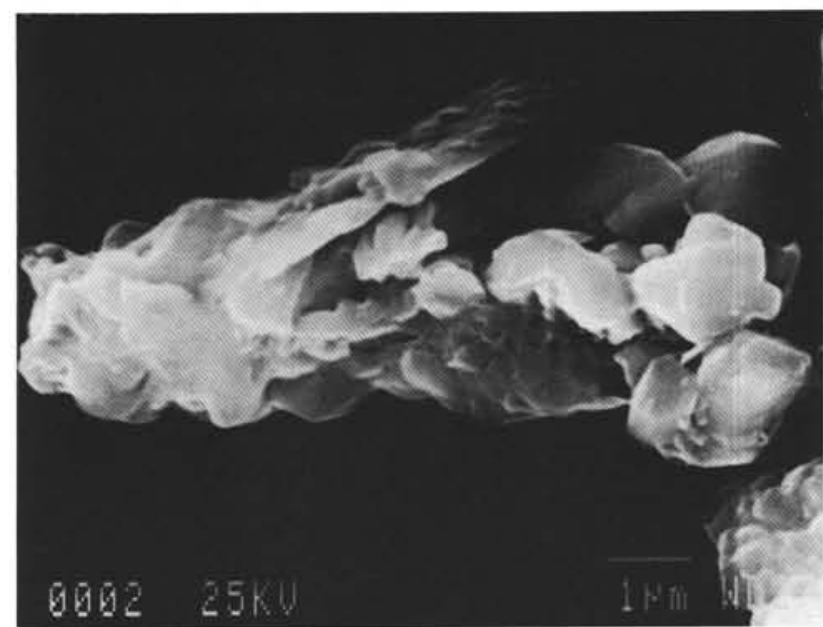

5



7

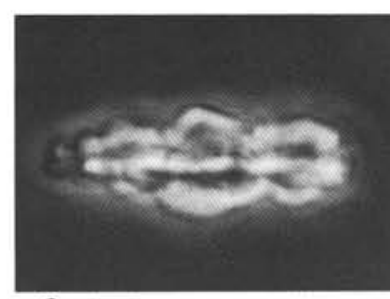

9

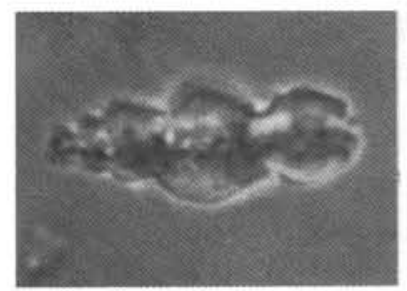

11

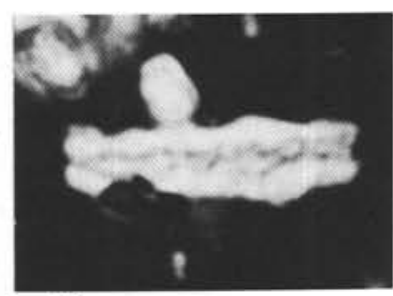

10

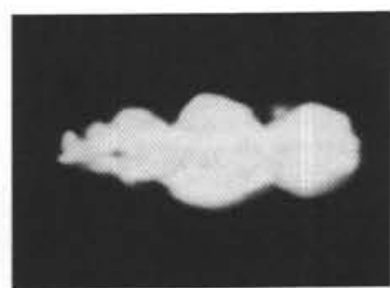

12

Plate 14. 1-12. Lithraphidites bollii (Thierstein), $(1,2 \text {, and 4) })^{\mathrm{s} 5}$ Sample 103-638B-27R-5, 54-55 cm, (1, Pol; 2, Ph; 4, Tr), (5) $8500 \times .\left(3^{\mathrm{s} 6}, 6\right) \mathrm{Sample}$ 103-638B-25R-4, 48-49 cm, (3, Ph, 2700×; 6, 5200×), (7, 10 $0^{\text {s7 }}$ ) Sample 103-638B-23R-5, 89-90 cm, (7, 6800×; 10, Pol, 2400×). (8) Sample 103638B-23R-5, 89-90 cm, 6500×. $(9,11 \text {, and } 12)^{s 8}, 5200 \times,(9, \mathrm{Ph} ; 11, \mathrm{Tr} ; 12, \mathrm{Pol})$. 


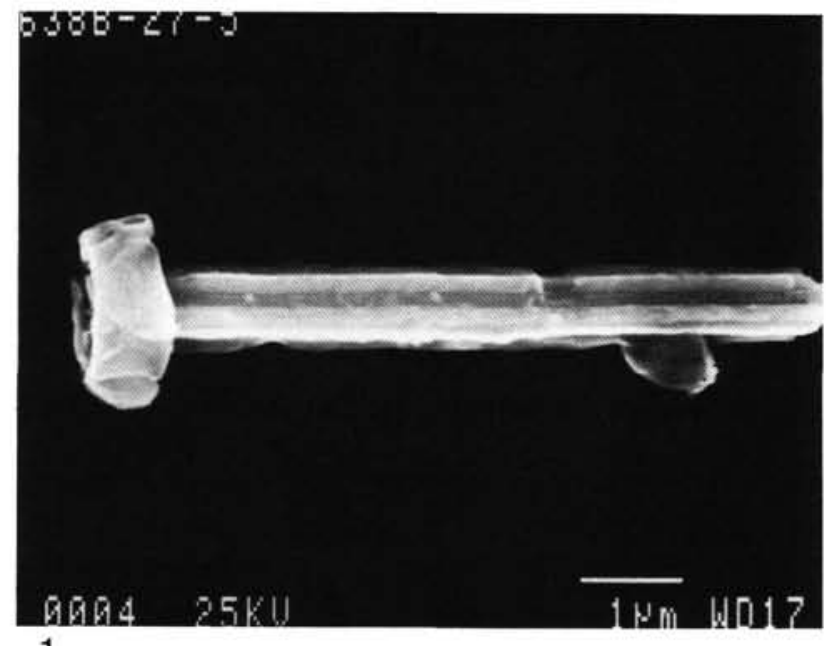

1

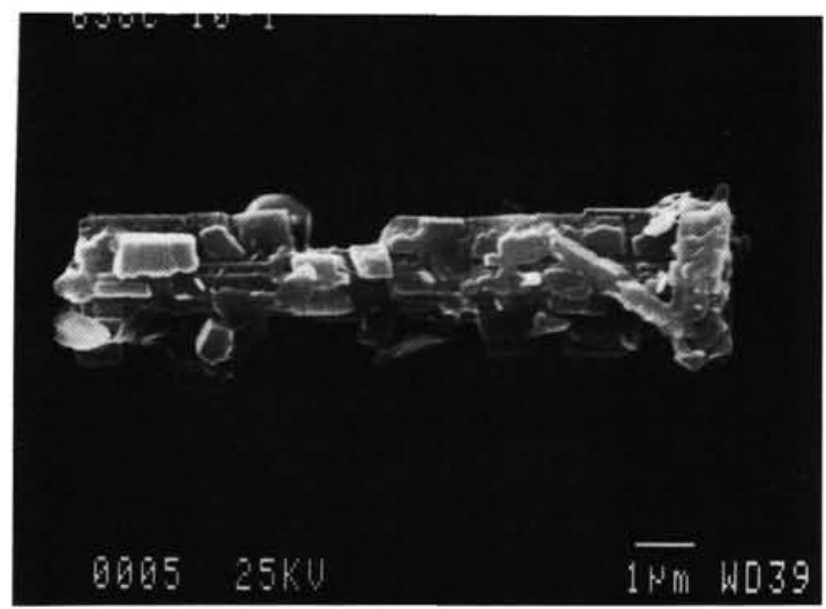

6

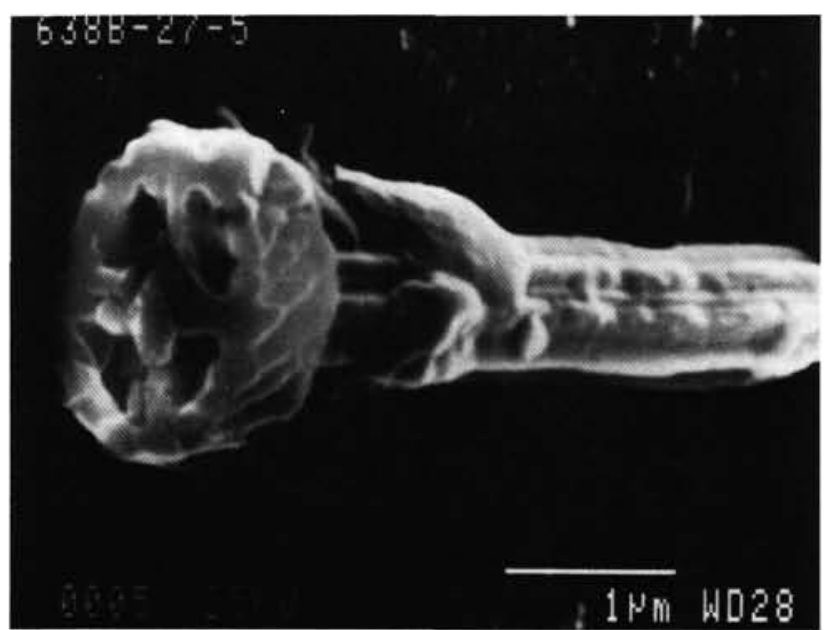

8

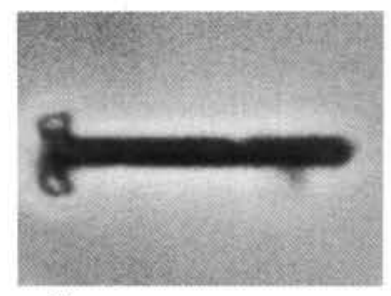

2

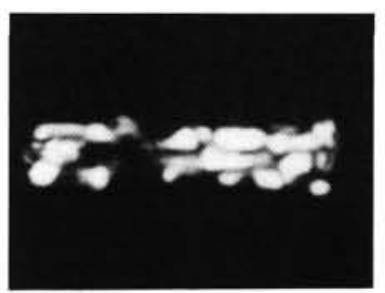

4

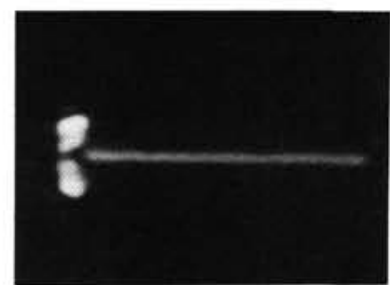

3

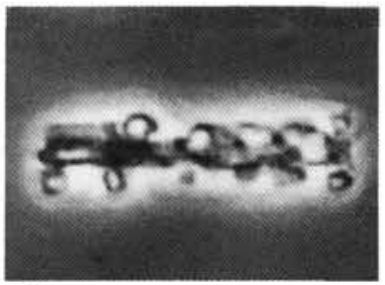

5

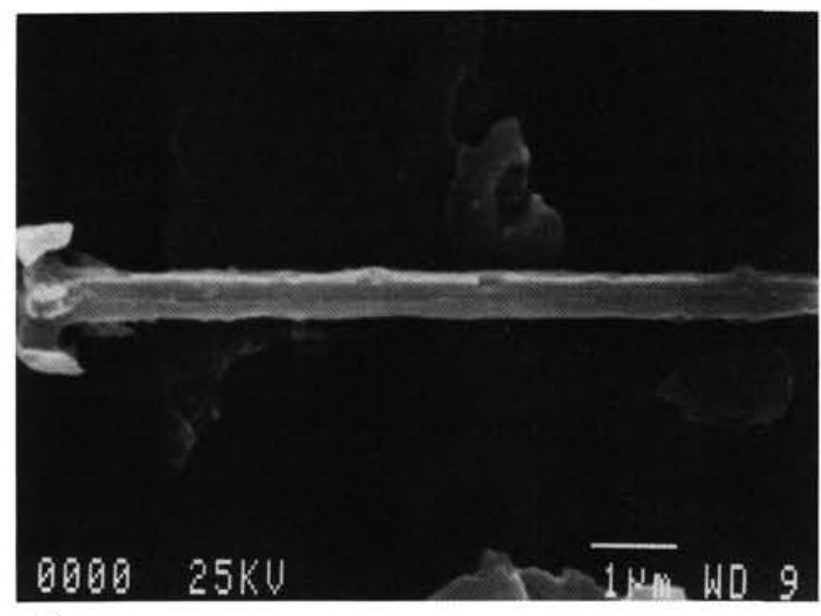

7

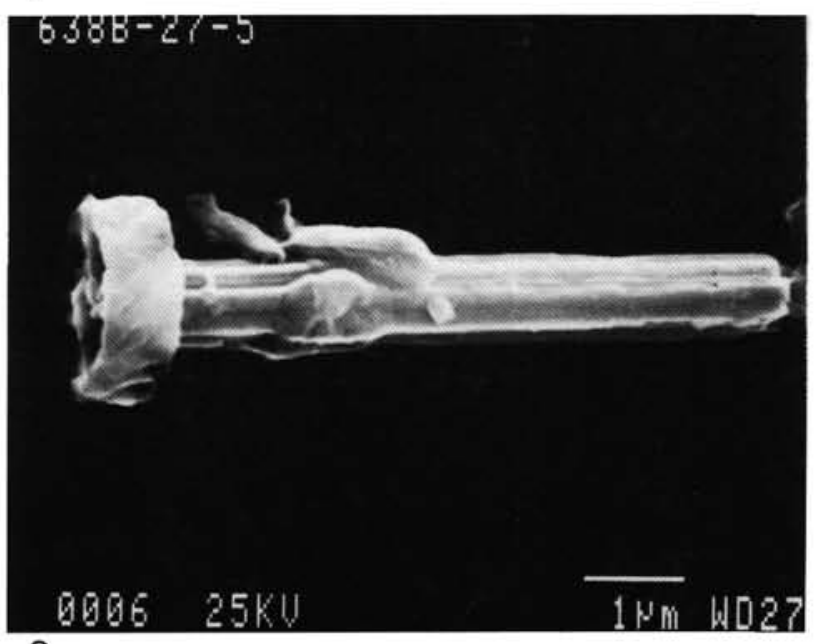

9

Plate 15. 1-9. Vekshinella parallela (Wind and Cepek) (1) Sample 103-638B-27R-5, 54-55 cm, L, 10,200× (2 and 3) $)^{\mathrm{s1}} 400 \times$, (2, Tr; 3 , Pol). (4 and $5)^{\mathrm{s} 6}$, Sample 103-638C-10R-1, 35-36 cm, 4000×, (4, Tr; 5, Pol), (6) L, 6000×. (7) Sample 103-638B-27R-5, 54-55 cm, L 8800×, (8-9) Sample 103638B-27R-5, 54-55 cm, (8, oblique P, 17,200×; 9, L, 10,000 $)$. 

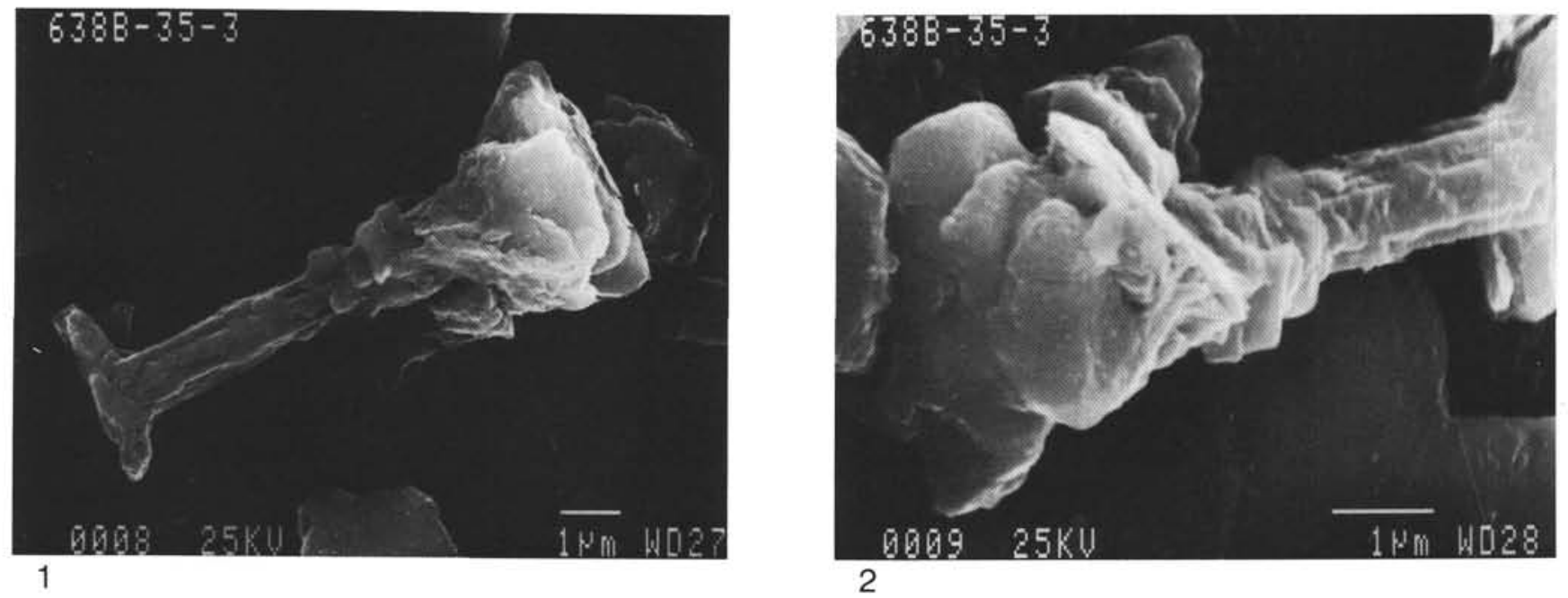
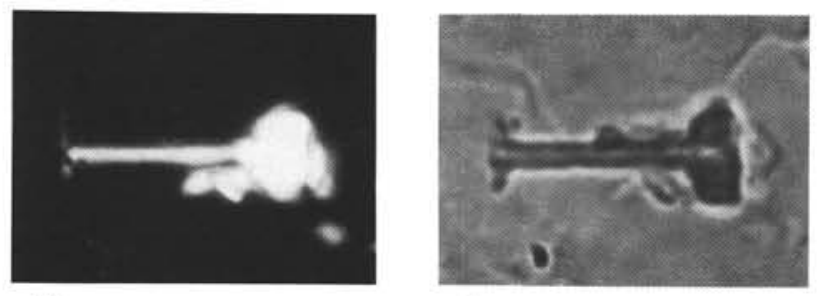

3

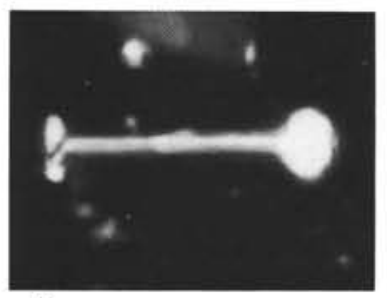

5

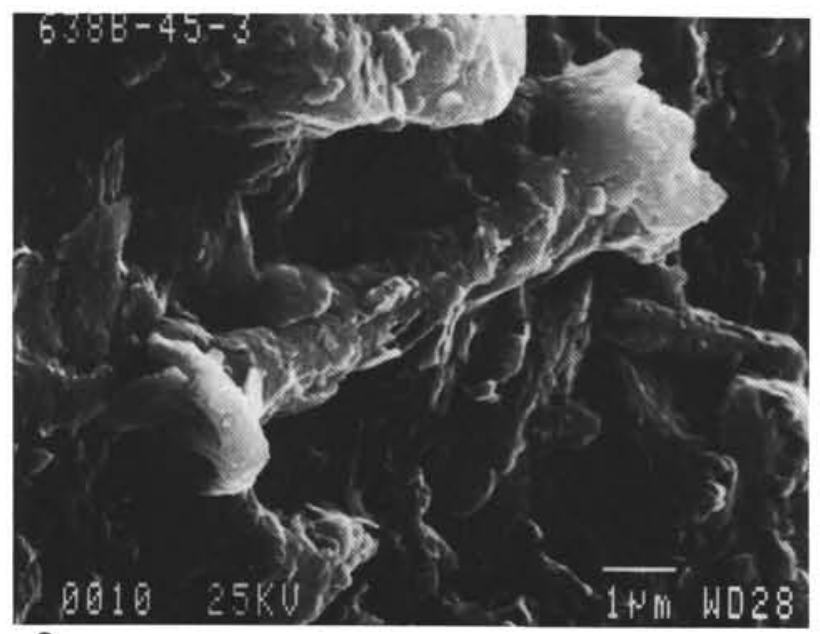

8

\section{4}

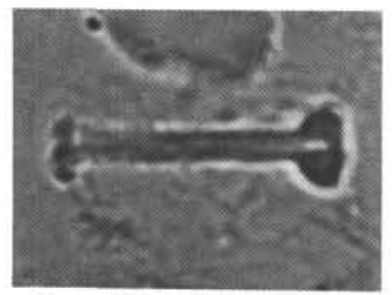

6

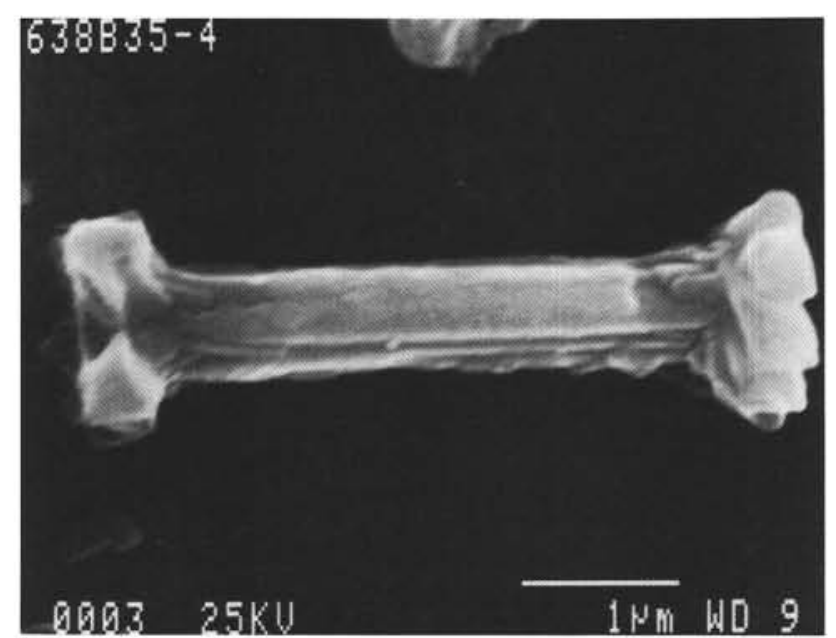

7

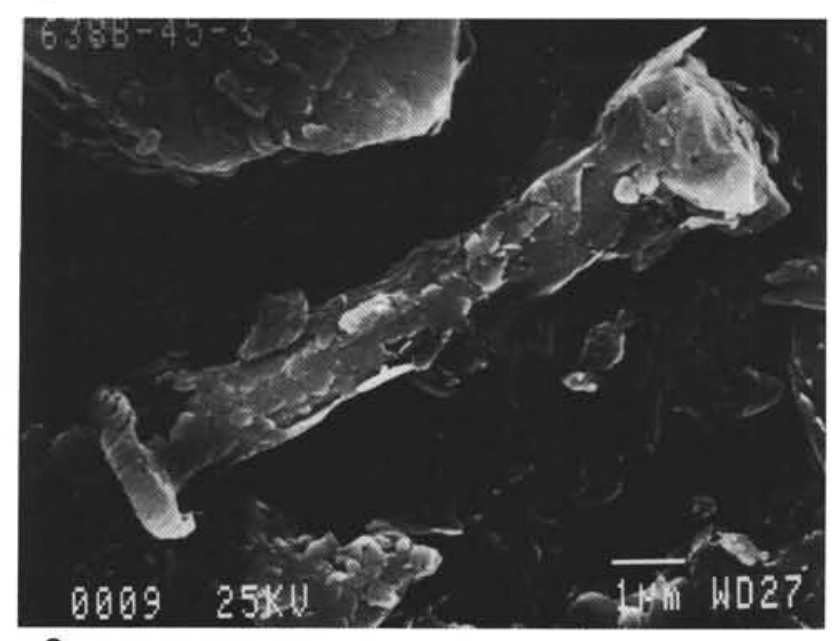

9

Plate 16. 1-9. Vekshinella pseudocarinolithus Applegate and Bergen n. sp. (1-4) Sample 103-638B-35R-3, 111-112 cm, (1, L, 6800 $\times$; 2, oblique D, $11,400 \times,(4)^{51}, 2600 \times,\left(3\right.$, Pol; 4, Tr) $, 5,6,8$, and 9) Sample 103-638B-45R-3, $28-29 \mathrm{~cm},\left(5\right.$ and 6) ${ }^{58}$ holotype, 2800 $\times,(5$, Pol; 6, Tr), (8) oblique P, $7500 \times$, (9) $\mathrm{L}, 7500 \times$. 


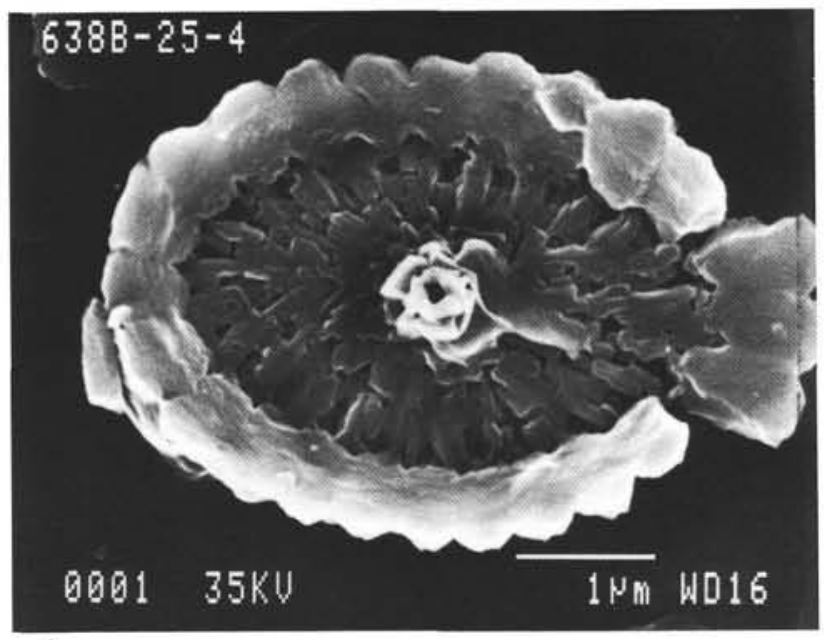

1

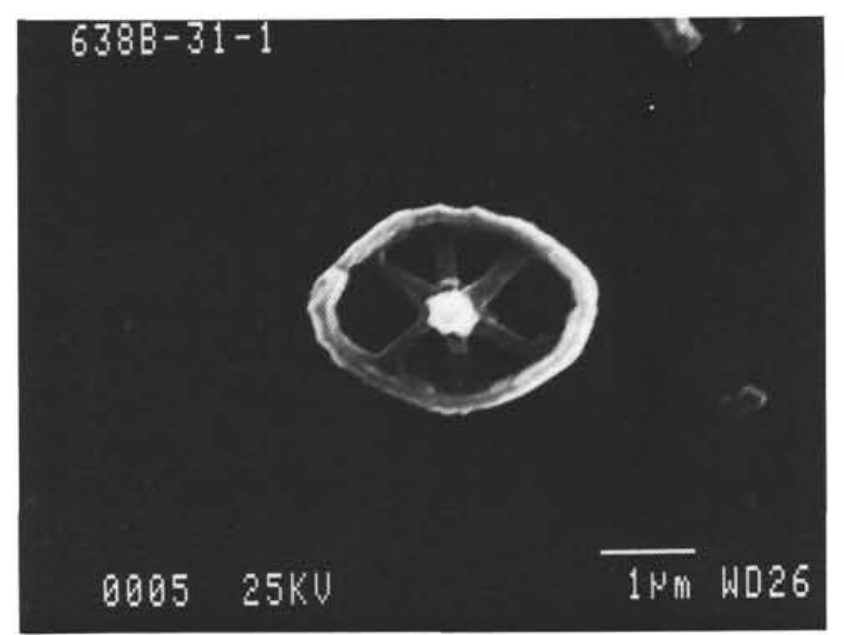

3

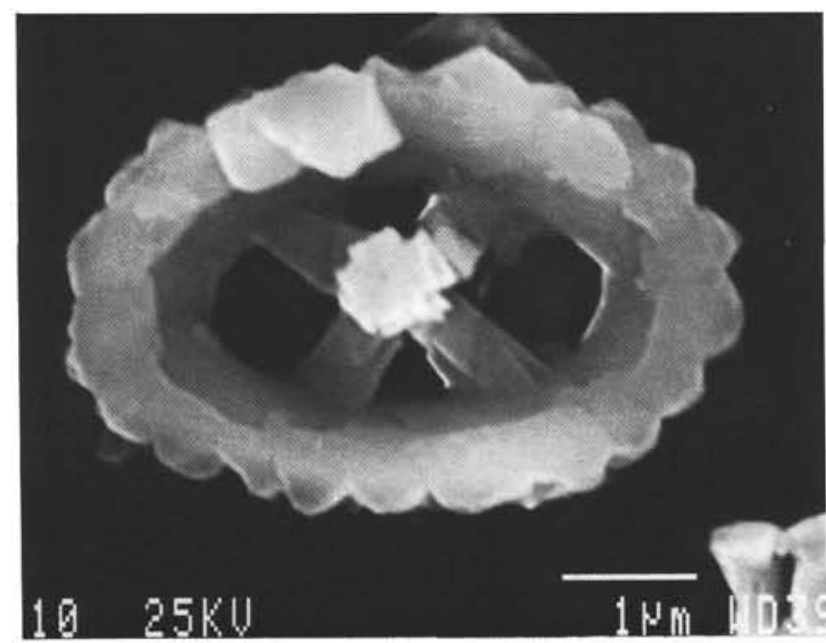

5

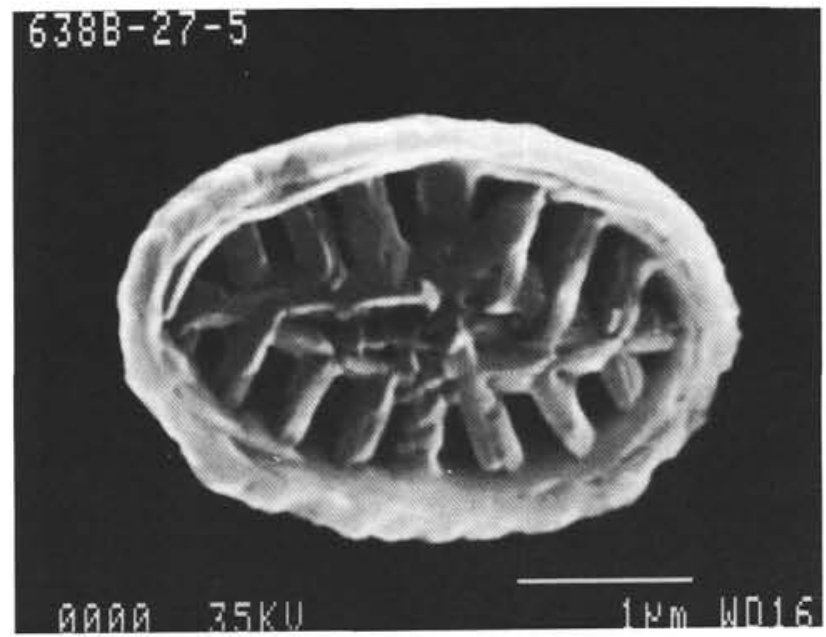

2

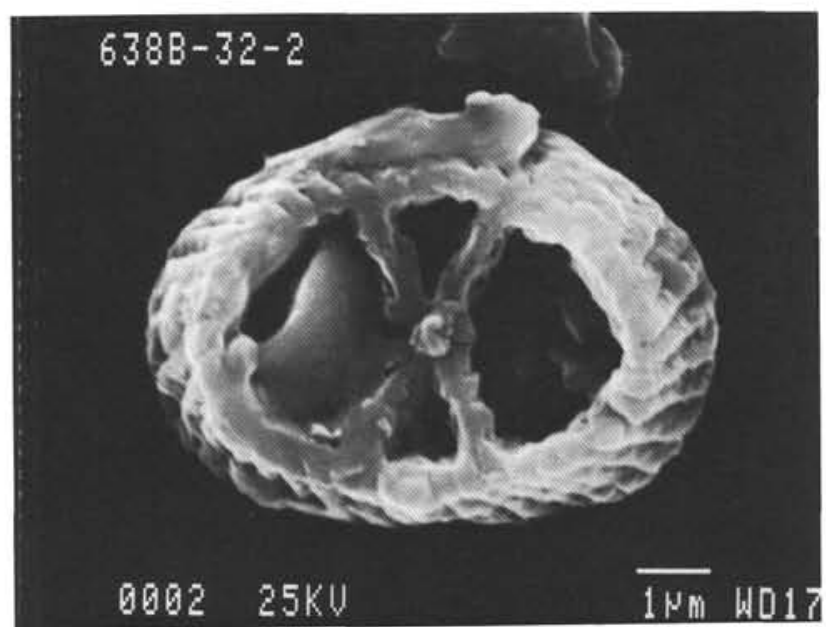

4

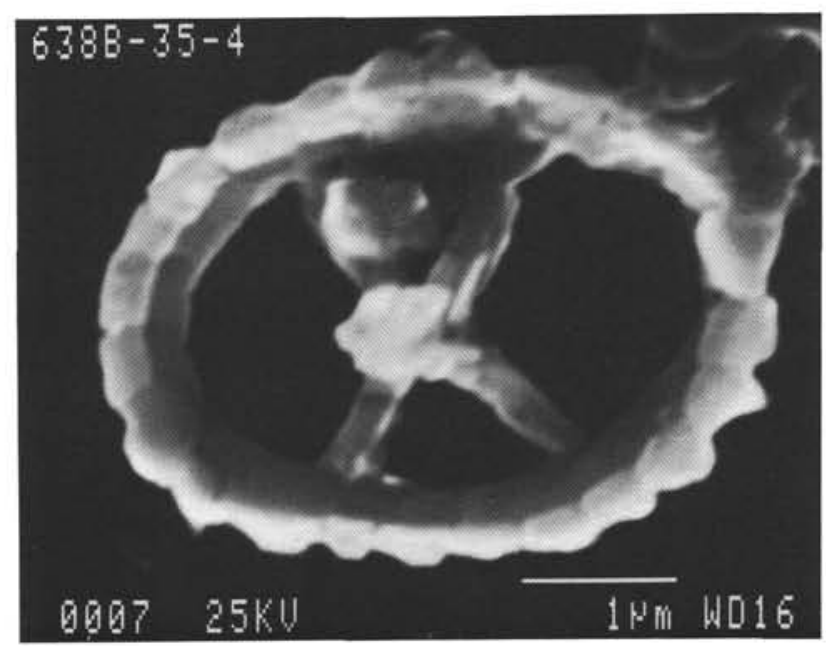

6

Plate 17. 1. Nodosella silvaradion (Filewicz), Sample 103-638B-25R-4, $48-49 \mathrm{~cm}, \mathrm{D}, 14,200 \times$. 2. Thurmannolithion clatratum Grun and Zweili, Sample 103-638B-27R-5, 54-55 cm, D, 17,900×. 3. Corollithion ellipticum Bukry, Sample 103-638B-31R-1, 125-126 cm, 9700×. 4-6. Corollithion acutum Thierstein, (4) Sample 103-638B-32R-2, 125-126 cm, P, 7500 × , (5 and 6) Sample 103-638B-35R-4, 34-35 cm, (5) D, 13,800 × , (6) D, $15,800 \times$. 


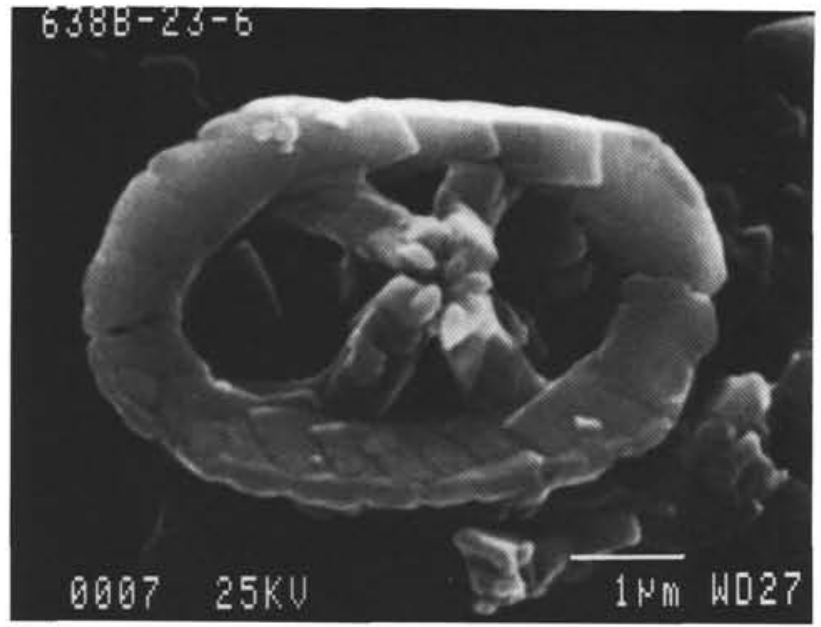

1

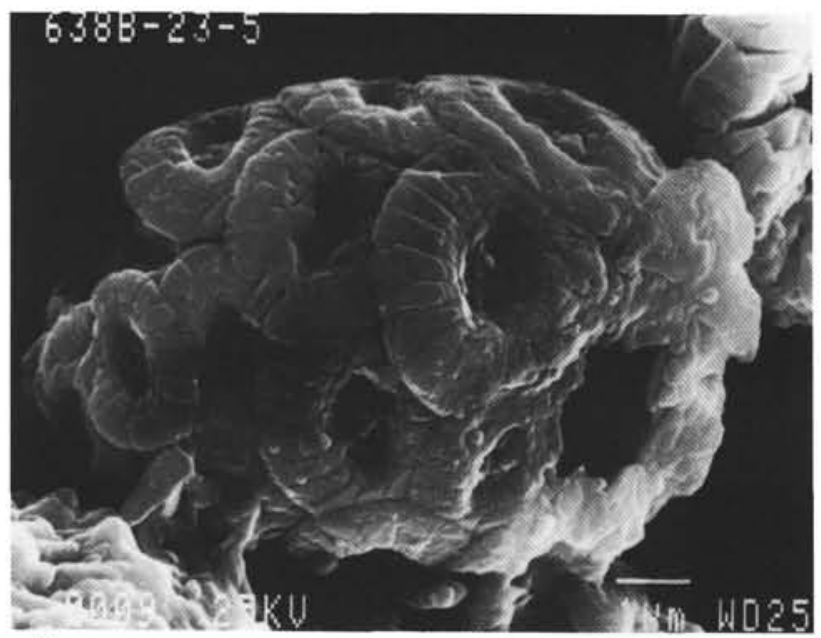

6

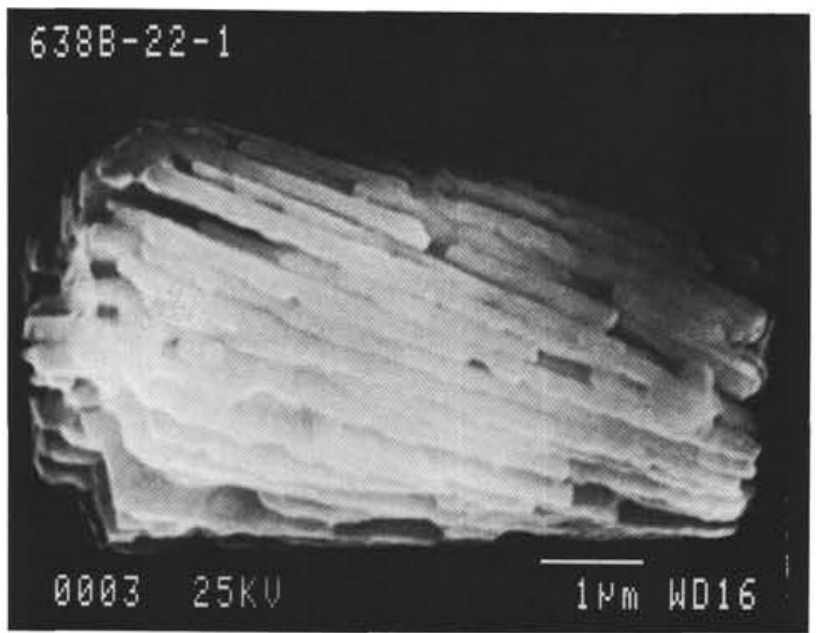

8



2

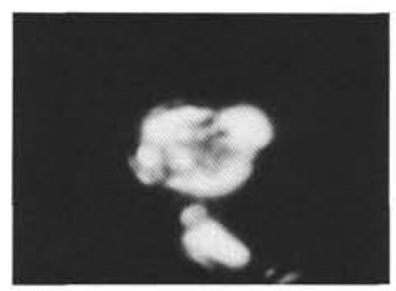

4

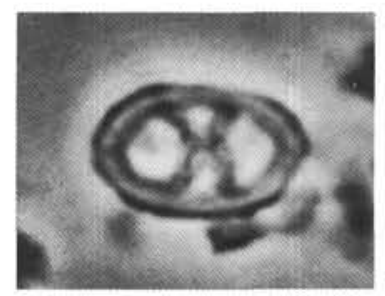

3

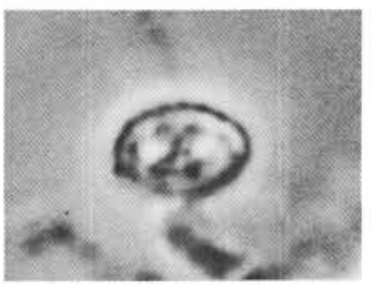

5



7

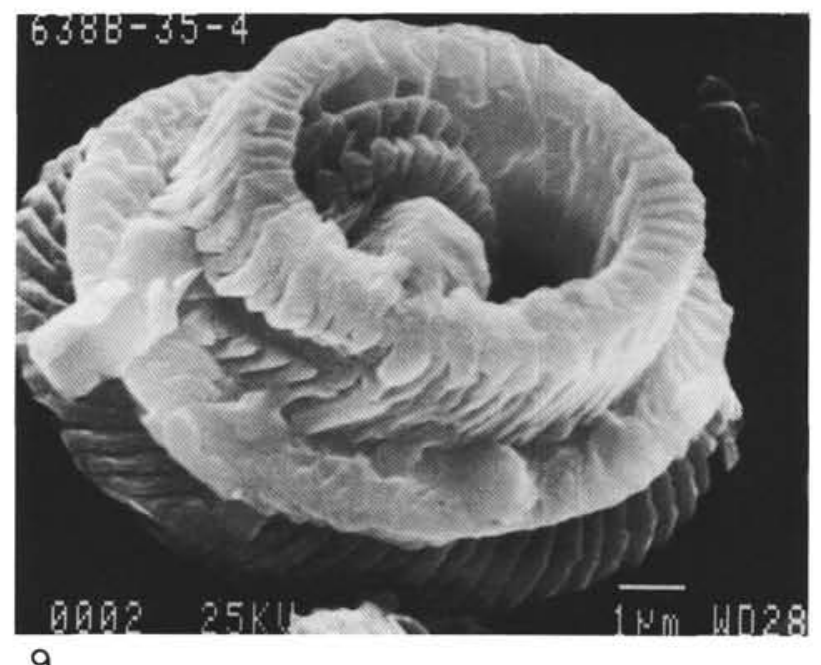

9

Plate 18. 1-3. Tegumentum stradneri Thierstein, Sample 103-638B-23R-6, 58-59 cm, (1) D, 11,500 $\times$, (2 and 3) ${ }^{\mathrm{s1}} 3900 \times,(2, \mathrm{Pol} ; 3, \mathrm{Ph})$. 4, 5, and 7. Corollithion acutum Thierstein, Sample 103-641A-6X, CC, $(4 \text { and 5) })^{57} 3600 \times,(4, \mathrm{Pol} ; 5, \mathrm{Ph}),(7) \mathrm{D}, 12,500 \times$. 6. Coccosphere, Biscutum constans (Gorka), Sample 103-638B-23R-5, 88-89 cm, 7800×. 8. Conusphaera mexicana Trejo, Sample 103-638B-22R-1, 41-42 cm. L, 10,500×. 9. Tubodiscus verenae Thierstein, Sample 103-638B-35-4, 34-35 cm, D, 7000 $\times$. 


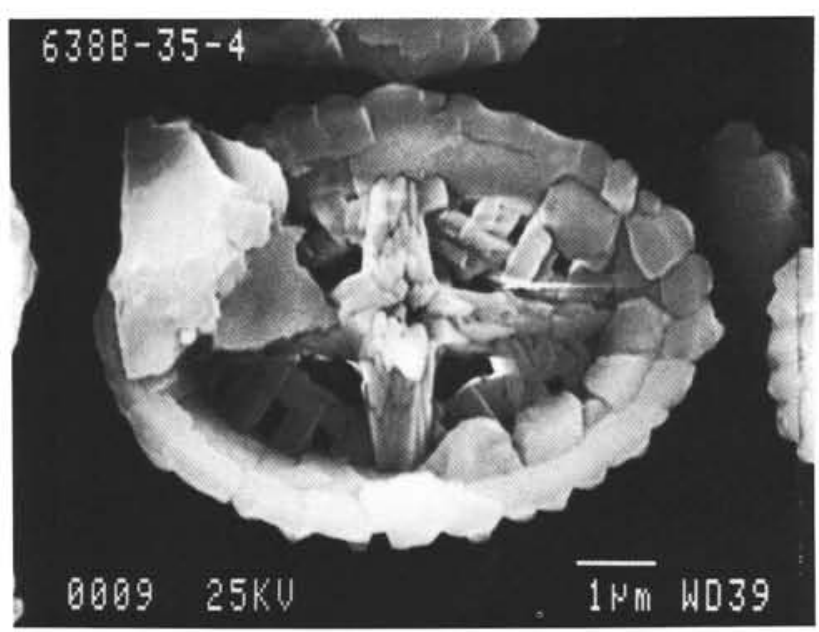

1

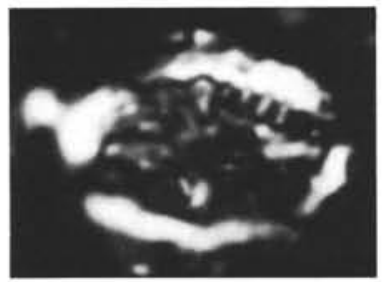

3

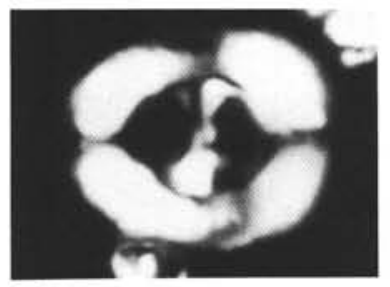

7

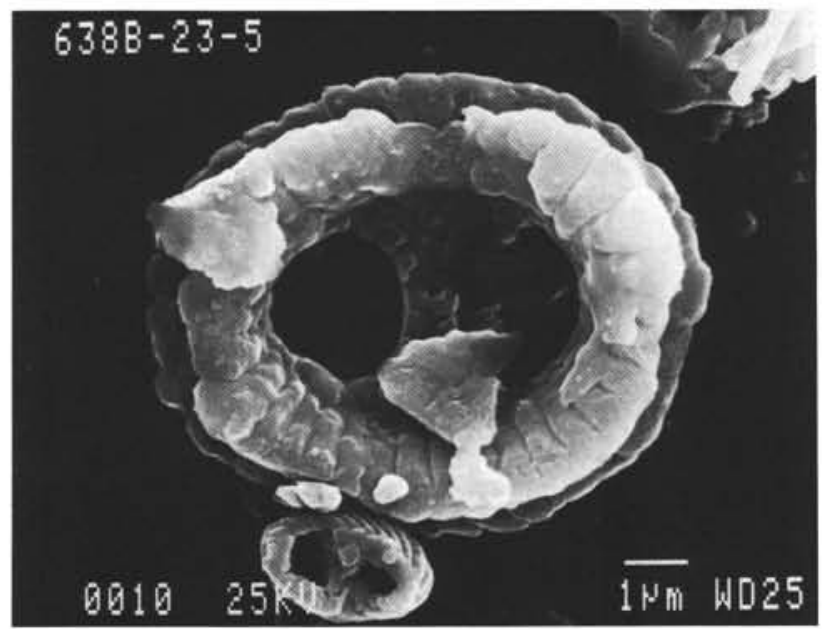

11



2



5

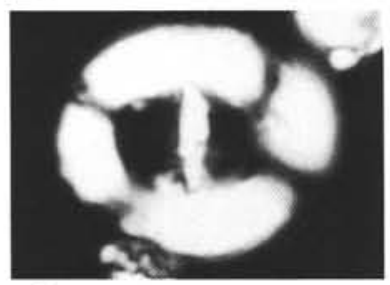

8

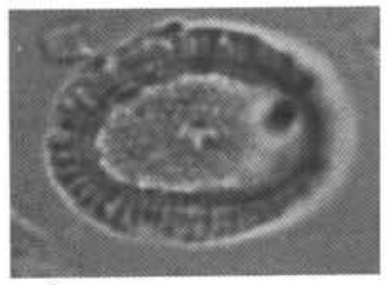

9

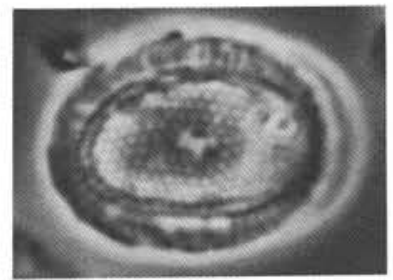

6

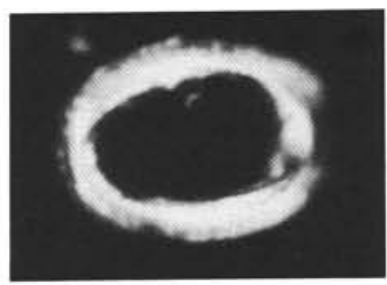

10



12

Plate 19. 1, 3, and 4. Diloma primitiva (Worsley), (1) Sample 103-638B-35R-4, 34-35 cm, D, 8000×. (3 and 4) Sample 103-638B-44R-1, 38-29 cm, $4100 \times,(3, \mathrm{Pol} ; 4, \mathrm{Tr}) .2$ and 5. Diloma sp. Wind and Cepek, Sample 103-638C-10R-1, 35-36 cm, (2) D, 8200 $\times .\left(5^{52}, \mathrm{Pol}, 2700 \times\right)$. 6, 9, 10, and 12. Ethmorhabdus hauterivianus (Black), Sample 103-638B-35R-3, 111-112 cm, (6, 9, and 10) ${ }^{\mathrm{s} 12} 2300 \times,(6, \mathrm{Ph} ; 9, \mathrm{Tr} ; 10, \mathrm{Pol})(12) \mathrm{P}, 6000 \times$. 7, 8, and 11. Speetonia colligata Black, Sample 103-638B-23R-5, $89-90 \mathrm{~cm}$, (7 and 8$)^{\text {s11 }} 3000 \times$, Pol, (11) P, $6300 \times$. 


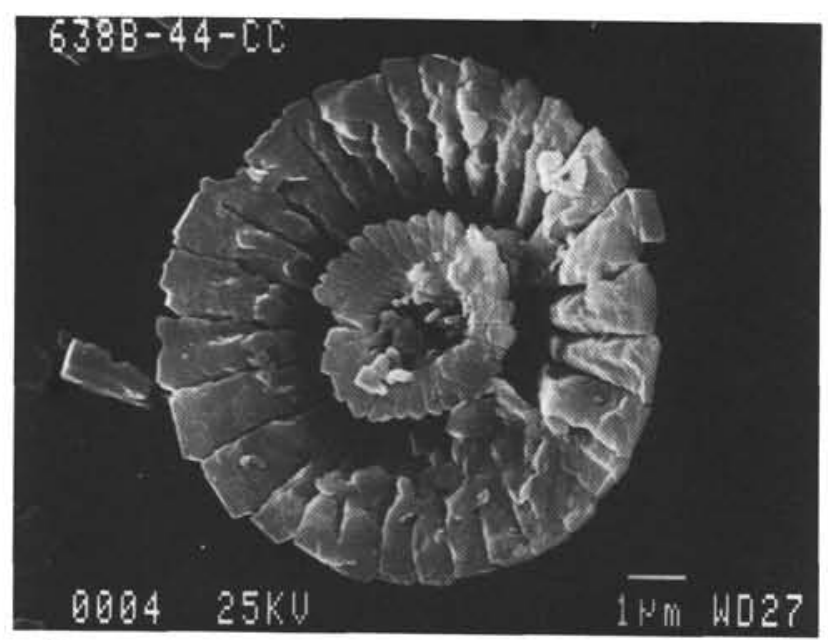

1

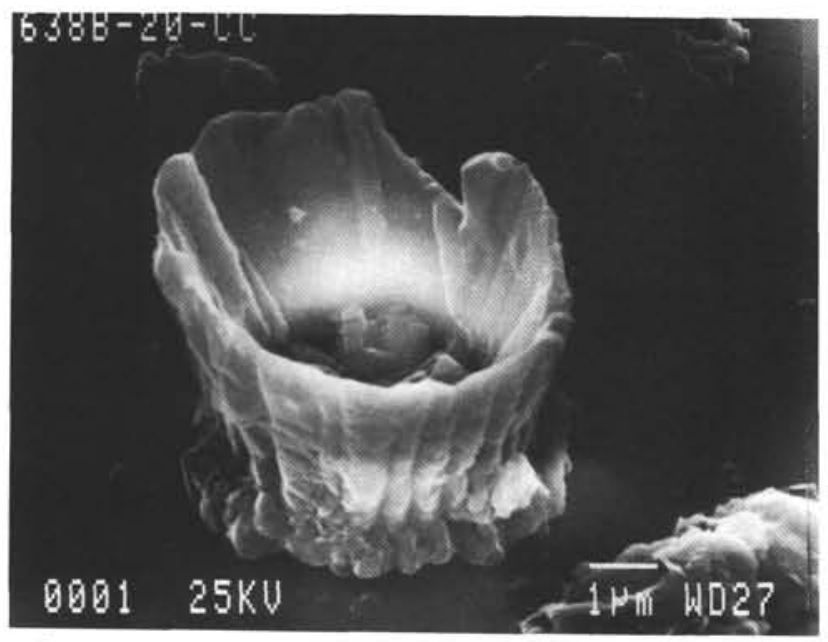

6



8

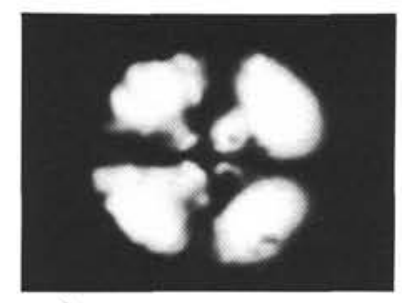

2

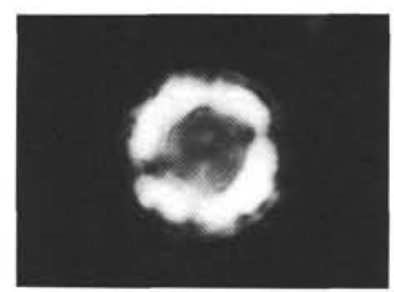

4

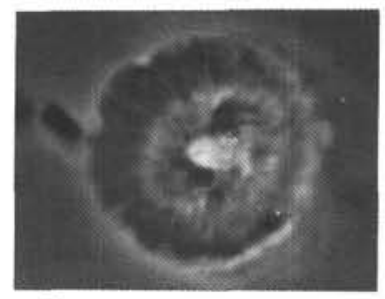

3

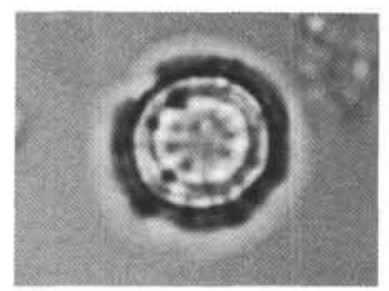

5

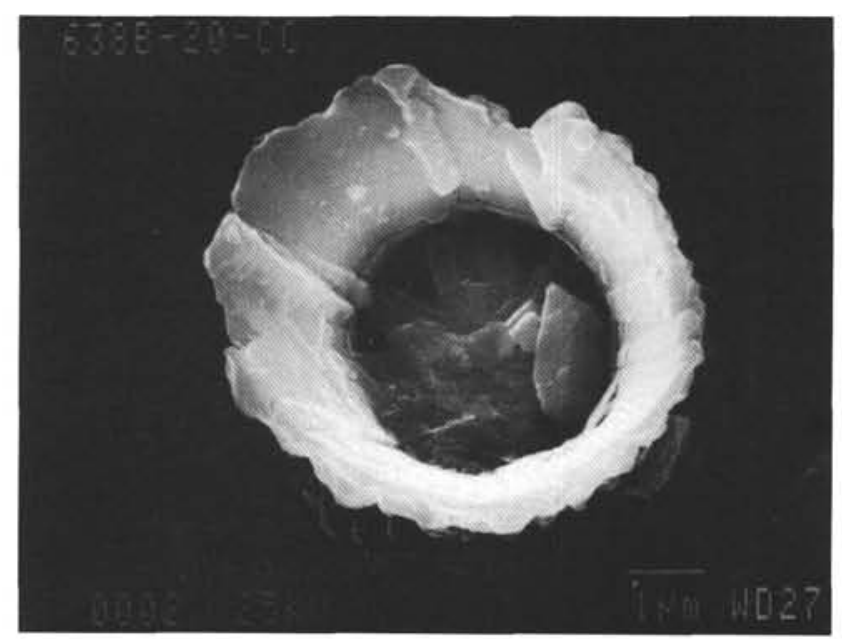

7

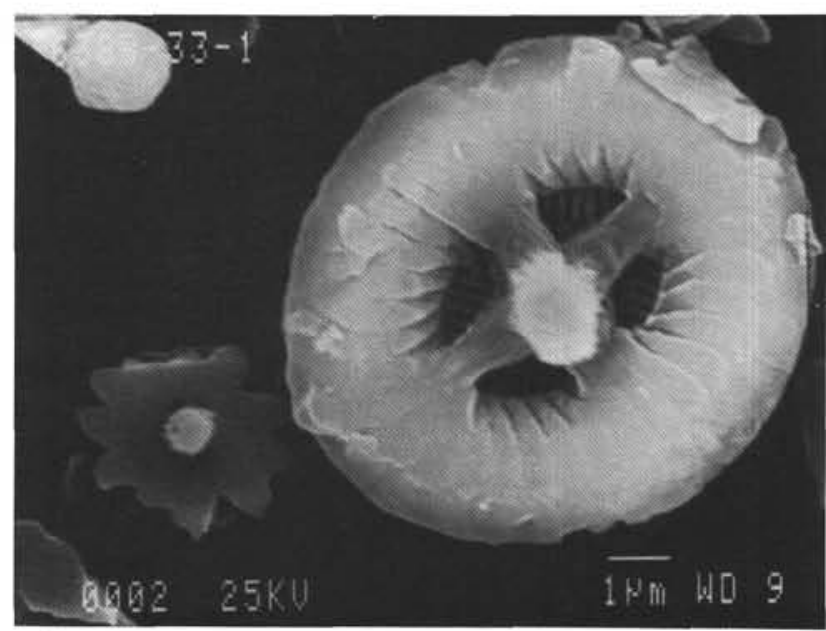

9

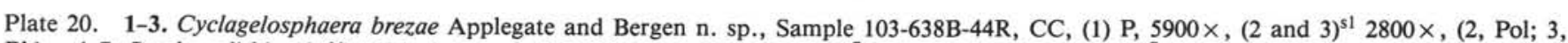
$\mathrm{Ph})$ 4-7. Stephanolithion laffittei Noel, Sample 103-638B-20R, CC, (4 and 5) ${ }^{\mathrm{s7}} 3100 \times,(4, \mathrm{Pol} ; 5, \mathrm{Tr}),(6)^{\mathrm{s} 7}$ oblique D, 6900 $\times$, (7) D, 7800 $\times$. 8. Discorhabdus biradiatus (Worsley), Sample 103-638B-35R-4, 34-35 cm, P, 14,500 × . 9. Cruciellipsis cuvillieri ssp. cuvillieri Manivit and Discorhabdus biradiatus (Worsley), Sample 103-638B-33R-1, 75-76 cm, D, $6200 \times$. 


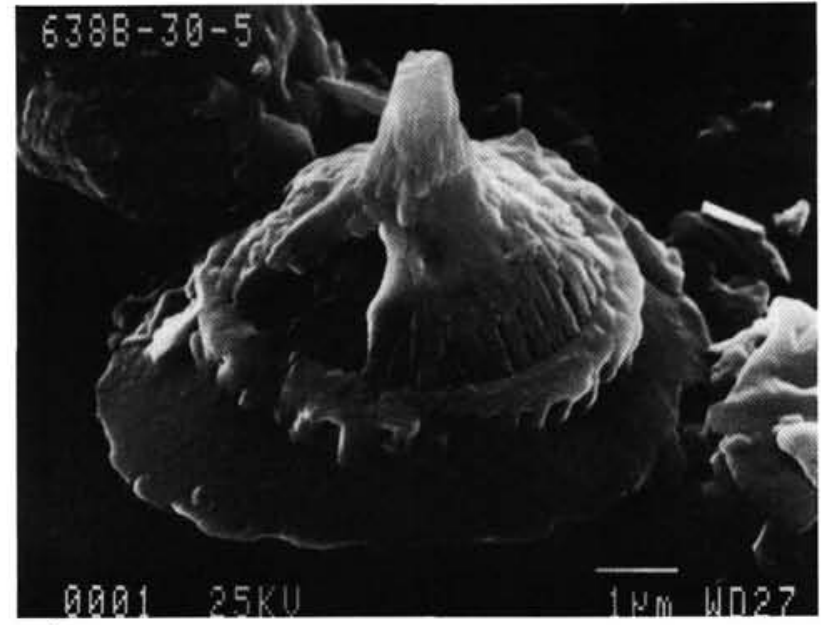

1



6

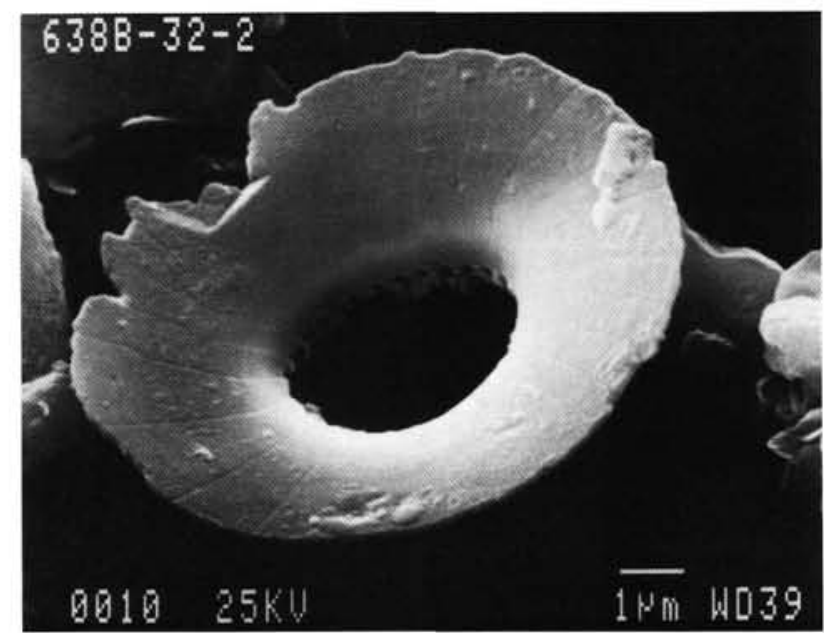

8

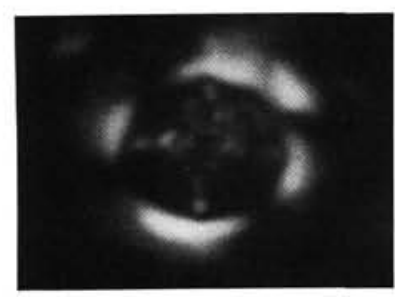

2

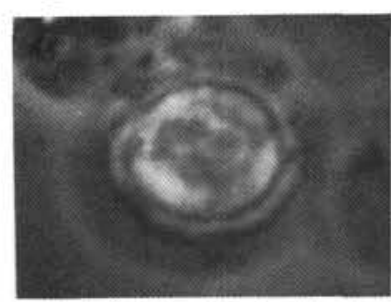

4

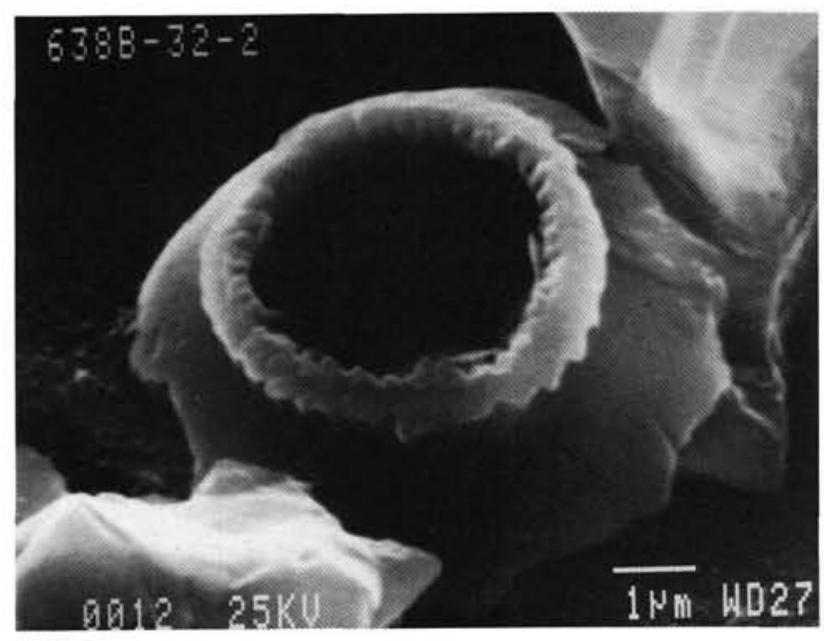

7

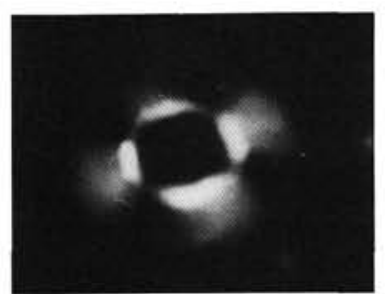

9

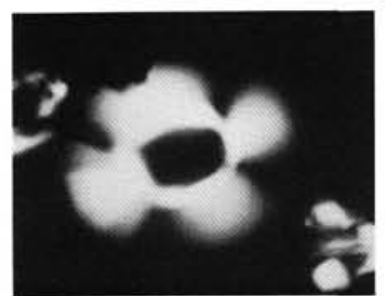

11

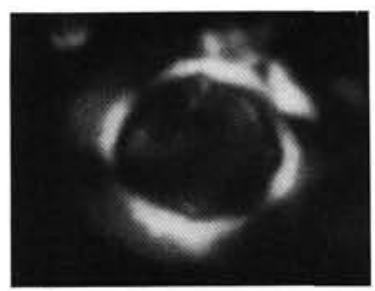

3



5

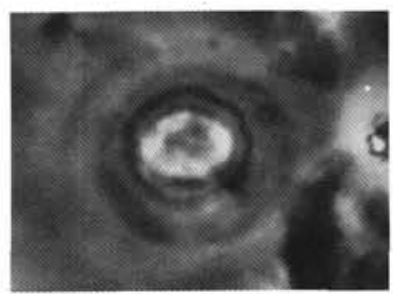

10



12

Plate 21. 1-12. Pickelhabube furtiva (Roth), (1-6) Sample 103-638B-30R-5, 5-6 cm, $(1)^{\mathrm{s} 6}$ oblique D, $8300 \times,(2-5)^{\mathrm{s} 6} 3700 \times$, $(2$, Pol, high focus; 3 , Pol, low focus; 4 , Tr, high focus; 5 , Tr, low focus), (6) D, $8500 \times$, (7-12) Sample 103-638B-32R-2, 126-127 cm, (7) oblique D, 8500 $\times$, (8) oblique P, $6500 \times,(9 \text { and } 10)^{57} 2800 \times,(9$, Pol; 10, Tr $), 11$ and 12$)^{58} 2500 \times,(11$, Pol; 12, Tr $)$. 


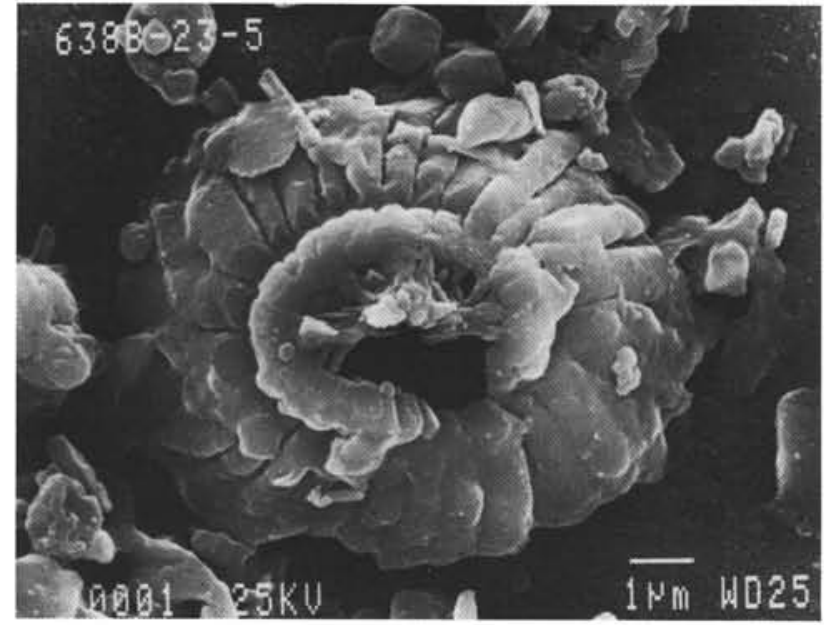

1

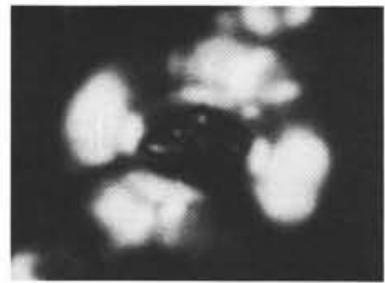

3

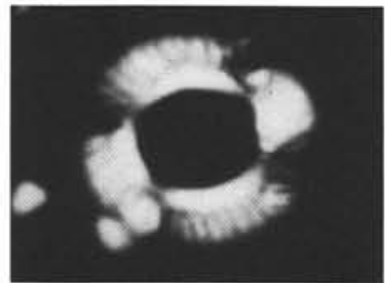

5

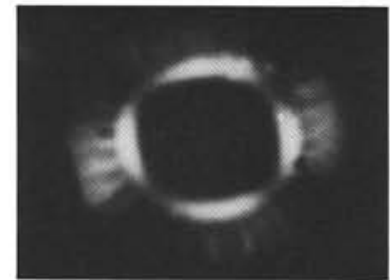

4

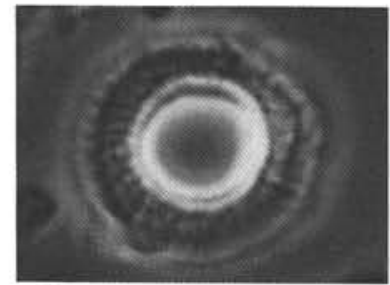

6

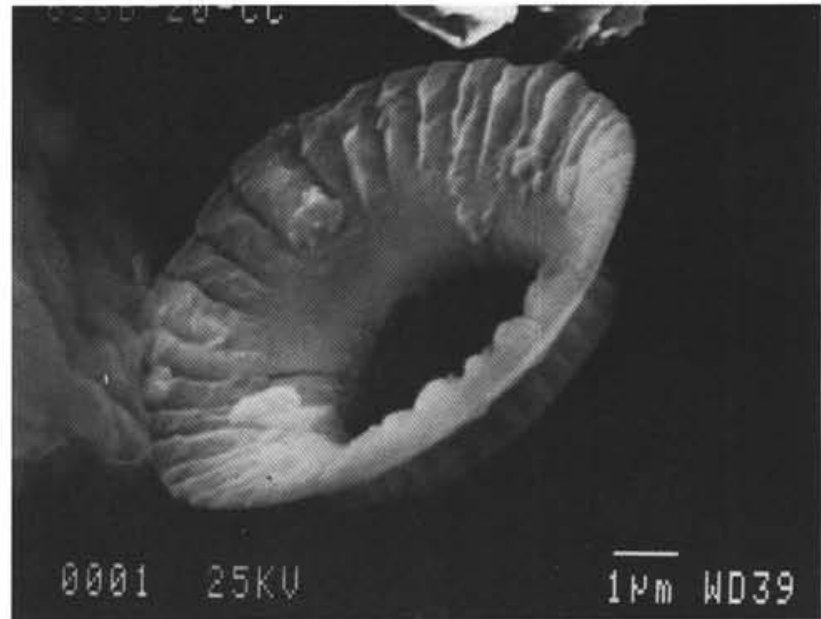

2

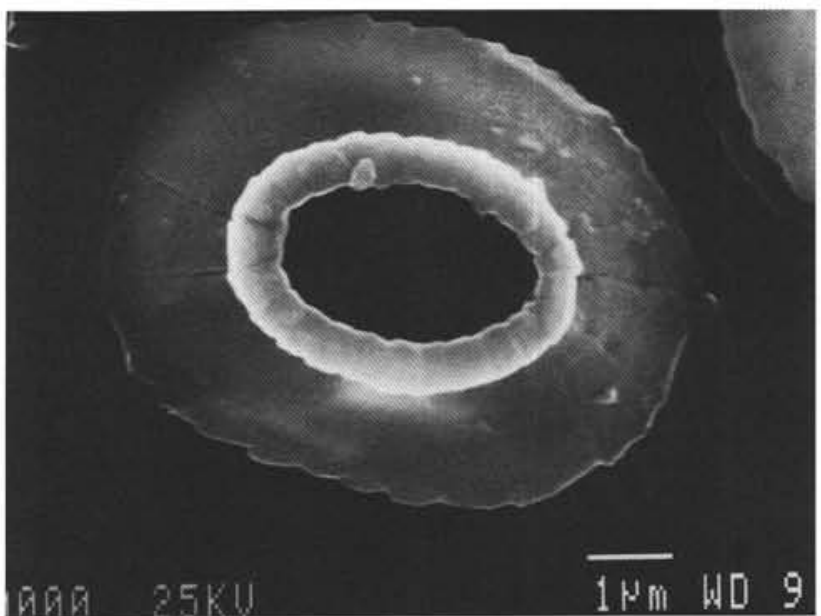

7



9

8

Plate 22. 1, 3, and 9 Pickelhaube furtiva (Roth), (1 and 3) Sample 103-638B-23R-5, 89-90 cm, (1) D, 6500 $\times$, (3) 31 300 $\times$, Pol, (9) Sample 103638B-32R-2, 126-127 cm, D, 7800 × 2, 4, and 7. Pickelhaube sp. 1. (2 and 4) Sample 103-638B-20R, CC, (2) oblique P, 6600 $\times,(4)^{s 2}$, Pol, 2800 $\times$, (7) Sample 103-638B-22R-1, 41-42 cm, D, $8600 \times$. 5, 6, and 8. Manivitella cf. M. pemmatoidea (Deflandre), Sample 103-638B-21R-2, 47-48 cm, (5 and 6) ${ }^{\mathrm{s}} 2400 \times,(5, \mathrm{Pol} ; 6, \mathrm{PhO},(8) \mathrm{P}, 6000 \times$. 


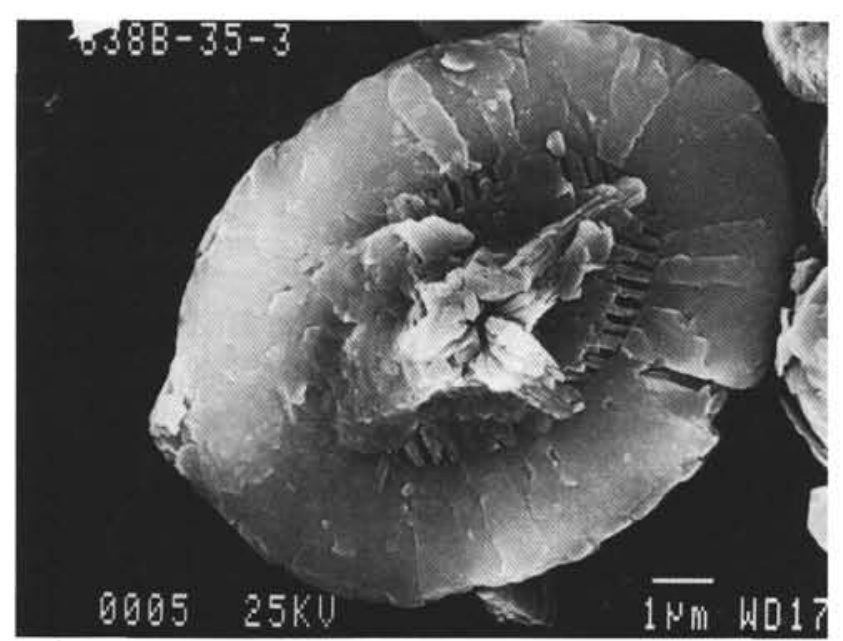

1

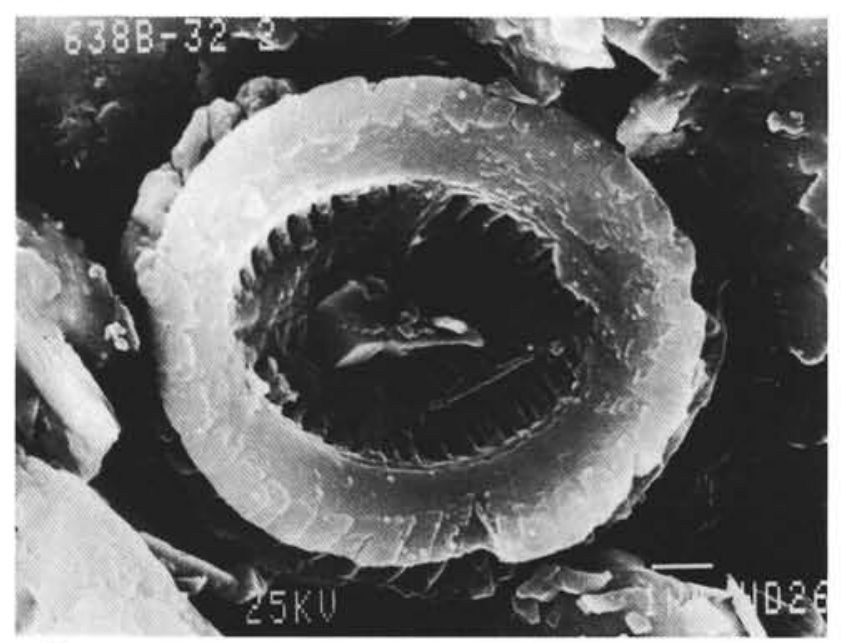

6

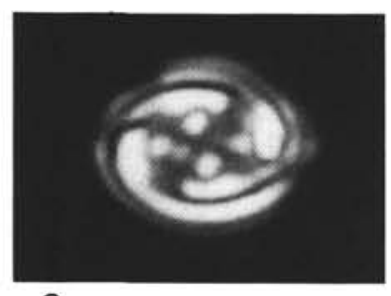

8

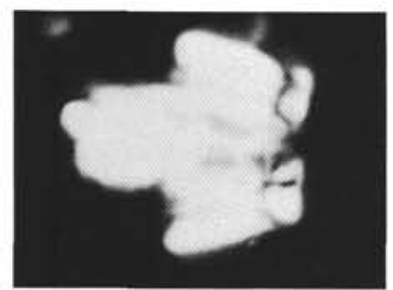

10

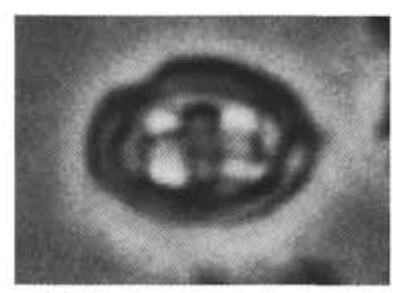

9

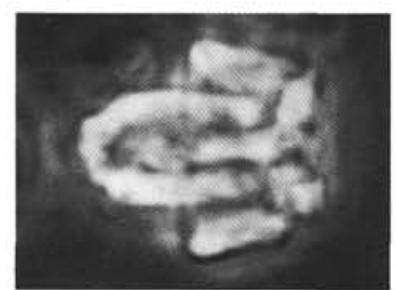

11

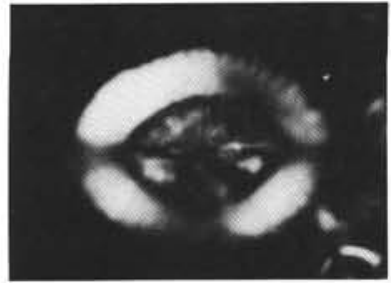

2

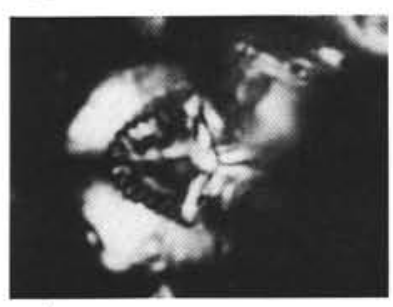

4

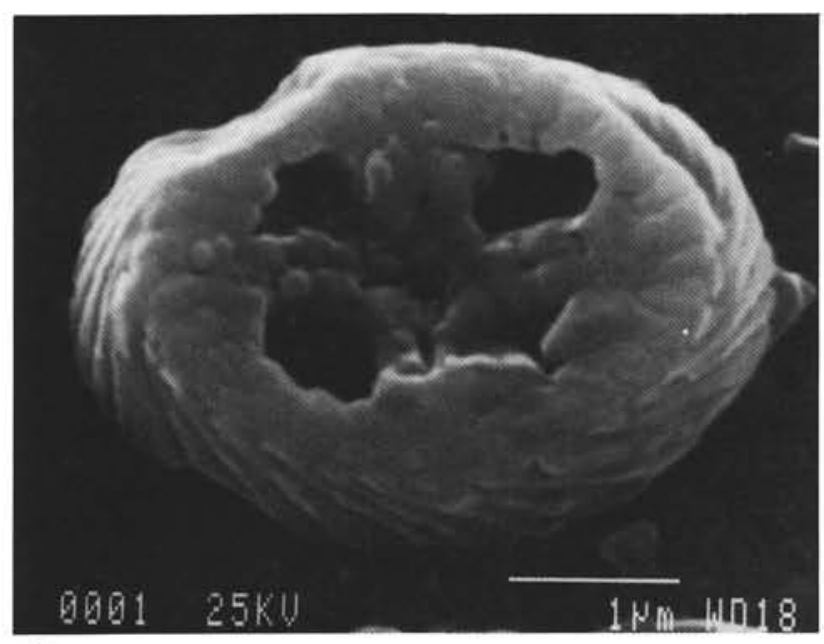

7

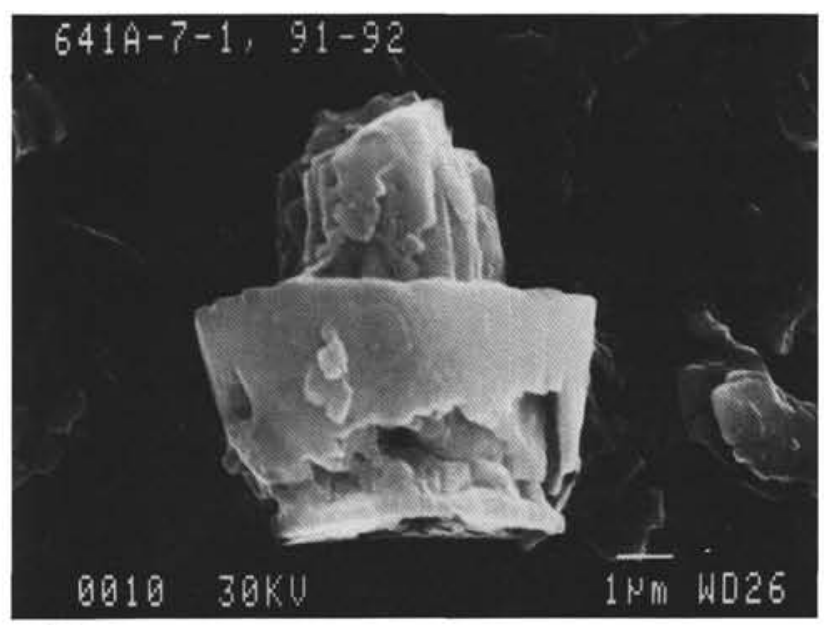

12

Plate 23. 1-6. Cretarhabdus delicatus Applegate, Covington, and Wise, (1-3) Sample 103-638B-35R-3, 111-112 cm, (1), D, 6200 $\times$, (2 and 3) $3000 \times, 92, \mathrm{Pol}, 45^{\circ}$ to $\mathrm{CN} ; 3, \mathrm{Pol}, 0^{\circ}$ to CN), (4-6) (Sample 103-638B-32R-2, 126-127 cm, (4) $2400 \times$, Pol, (5) 2300 $\times$, Tr, (6) P, 6100 $\times$. 7-9. Vekshinella mitcheneri Applegate and Bergen n. sp., Sample 103-638B-30R-5, 5-6 cm, (7) P, 17,400 $\times$, $(8-9)^{\mathrm{s} 7}$ 6400 $\times$, (8, Pol; 9 , Tr). 10-12. Parhabdolithus sp. Sample 103-641A-7X-1, 91-92 cm, (10 and 11) ${ }^{\text {s12 }} 2900 \times,(10$, Pol; 11, Ph), (12) L, 5800 $\times$. 




1

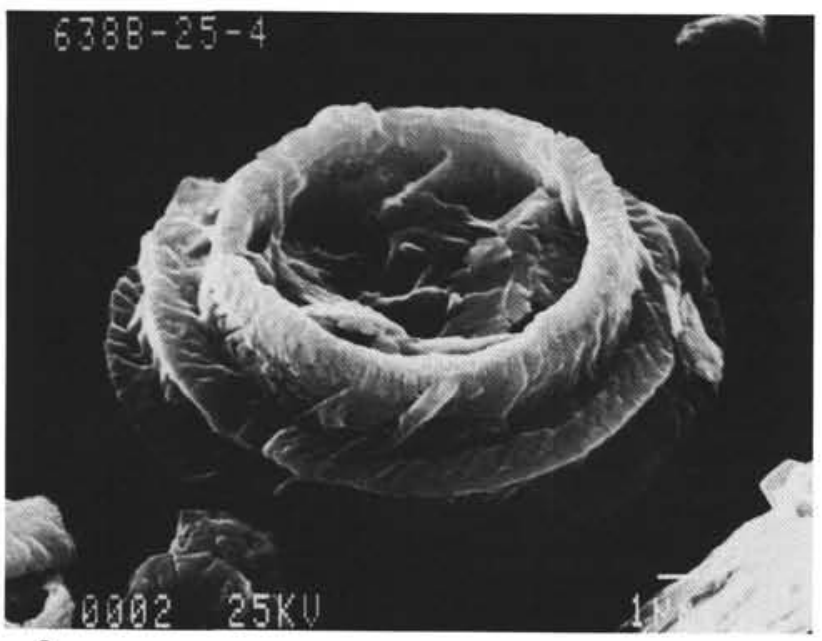

3

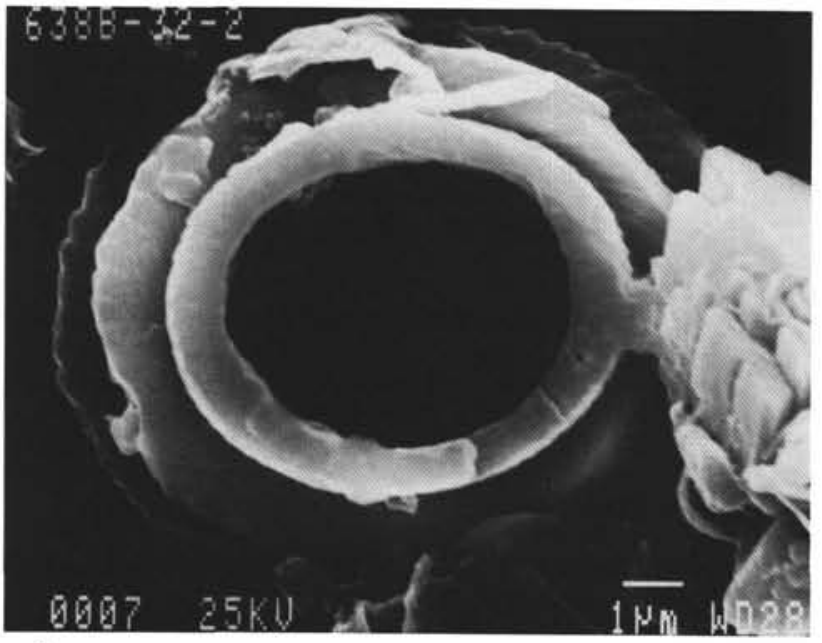
8

Plate 24. 1 and 2. Tubodiscus jurapelagicus (Worsley), Sample 103-638B-21R-2, 47-48 cm, (1) oblique D, 10,000×, (2) ${ }^{\mathrm{si}} \mathrm{P}, 7800 \times$. 3 and 6-9. Tubodiscus verenae Thierstein, (3) Sample 103-638B-25R-4, 48-48 cm, oblique D, 4400×, (6 and 7) ${ }^{53} 2600 \times$, (6, Pol; 7, Tr), (8 and 9) Sample 103$638 \mathrm{~B}-32 \mathrm{R}-2,126-127 \mathrm{~cm},(8)^{\mathrm{s}} \mathrm{D}, 6200 \times$, (9) oblique D, $6400 \times$. 4. Micrantholithus cf. obtusus Stradner, Sample 103-638C-10R-1, 35-36 cm, $2600 \times$, pol. 5. Lapideacassis sp. Sample 103-638B-22R-1, $41-42 \mathrm{~cm}, 2400 \times$, Tr.

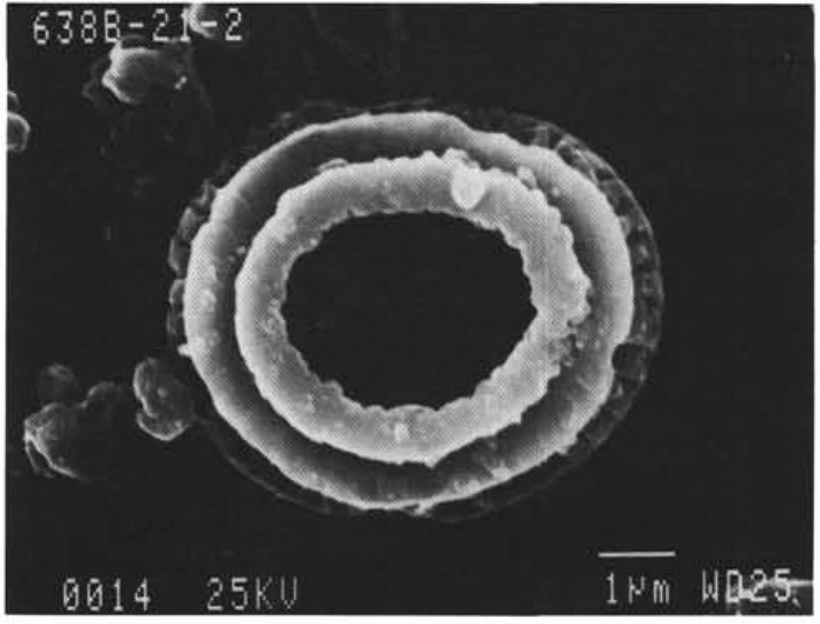

2



4

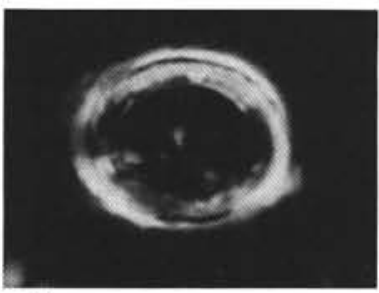

6

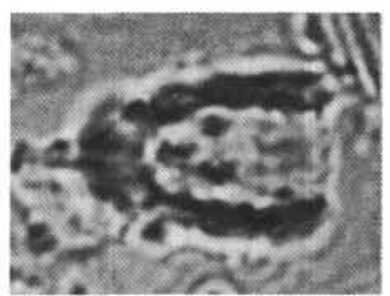

5

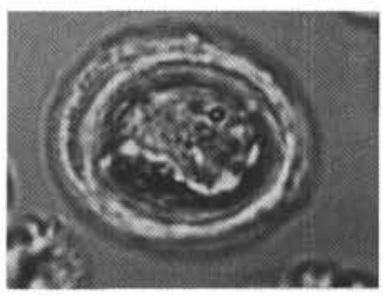

7

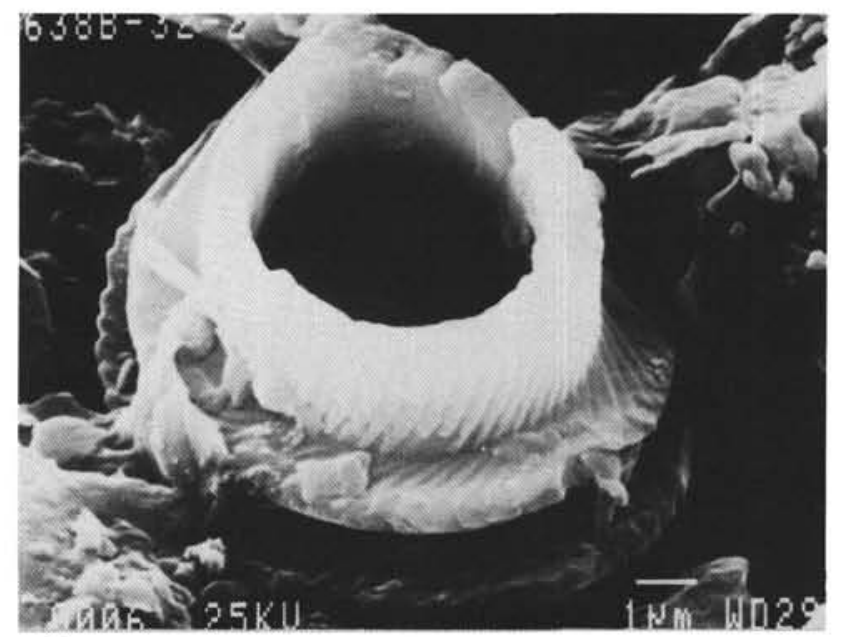




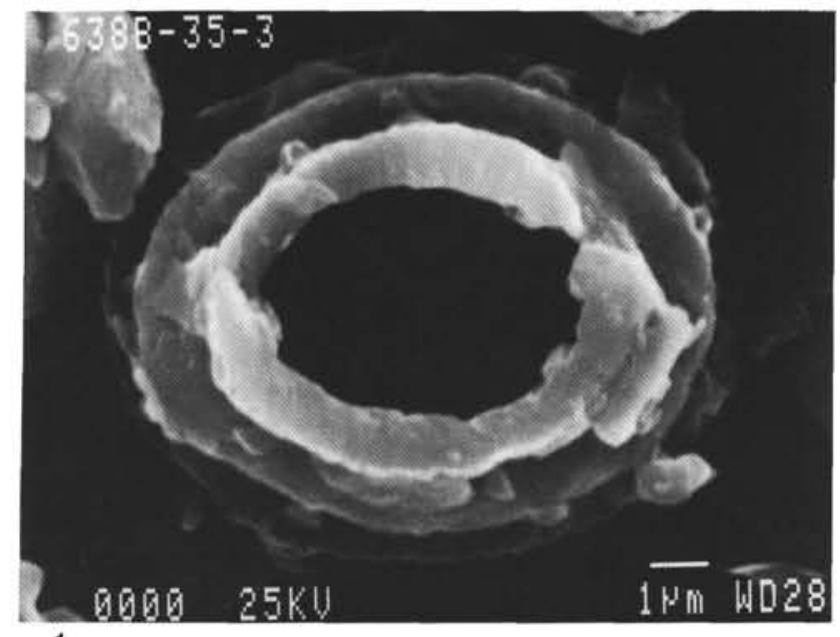

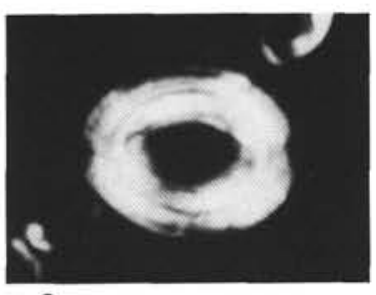

2

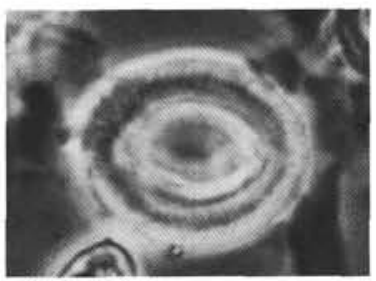

4

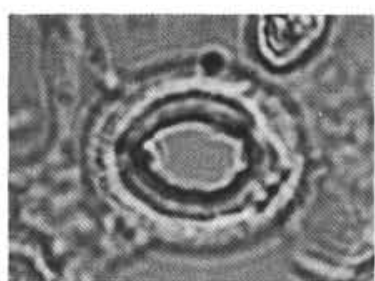

3

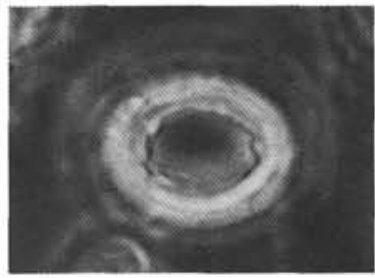

5

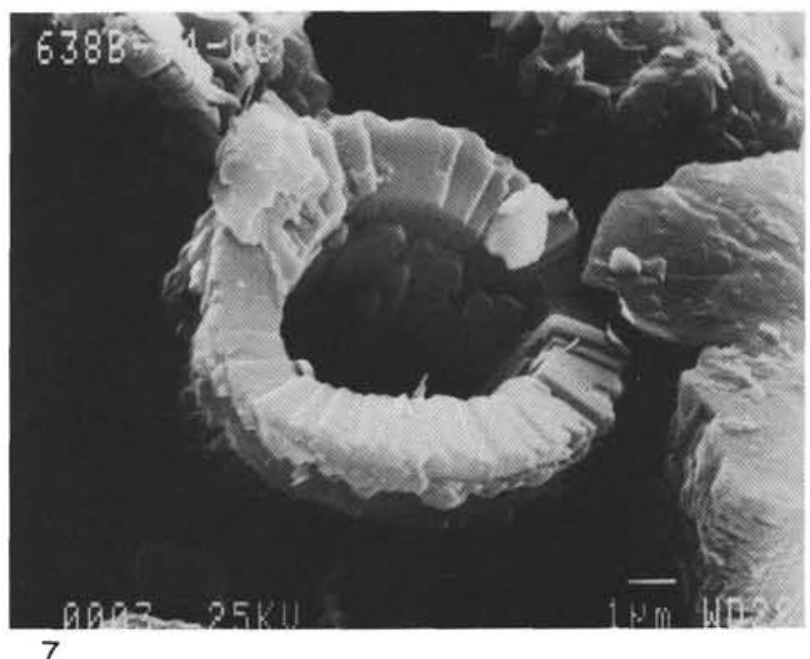

7

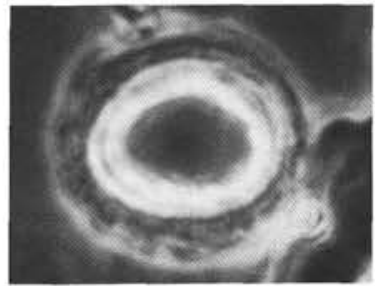

9

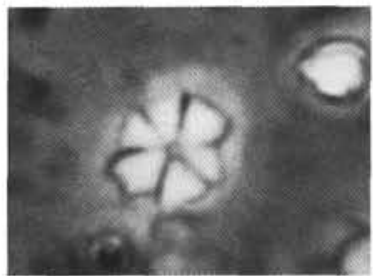

11

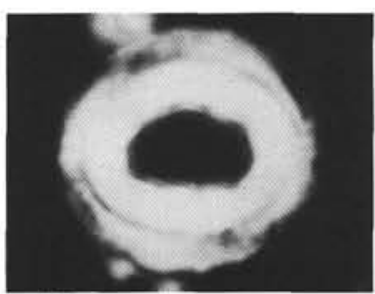

10

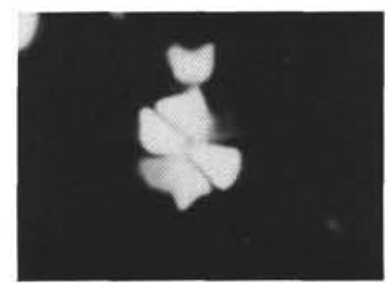

12 8

Plate 25. 1-10. Tubodiscus verenae Thierstein, (1-6) Sample 103-638B-35R-3, 111-112 cm, (1) D, 5900×, (2-5) ${ }^{\mathrm{s1}} 2100 \times$, (2, Pol; 3, Tr; 4, Ph low focus; $5, \mathrm{Ph}$, high focus), $(6)^{\mathrm{s1}}$ oblique D, $6100 \times(7-10)$ Sample $103-638 \mathrm{~B}-44 \mathrm{R}-1,38-39 \mathrm{~cm},(7)^{\mathrm{s}}$ oblique $\mathrm{P}, 5000 \times,(8) \mathrm{P}, 5100 \times,(9 \text { and } 10)^{58}$ $2300 \times,(9, \mathrm{Ph} ; 10, \mathrm{Pol}) .11$ and 12. Rucinolithus wisei Thierstein, Sample 103-638B-45R-2, 79-80 cm, 4600×, (11, Ph: 12, Pol). 

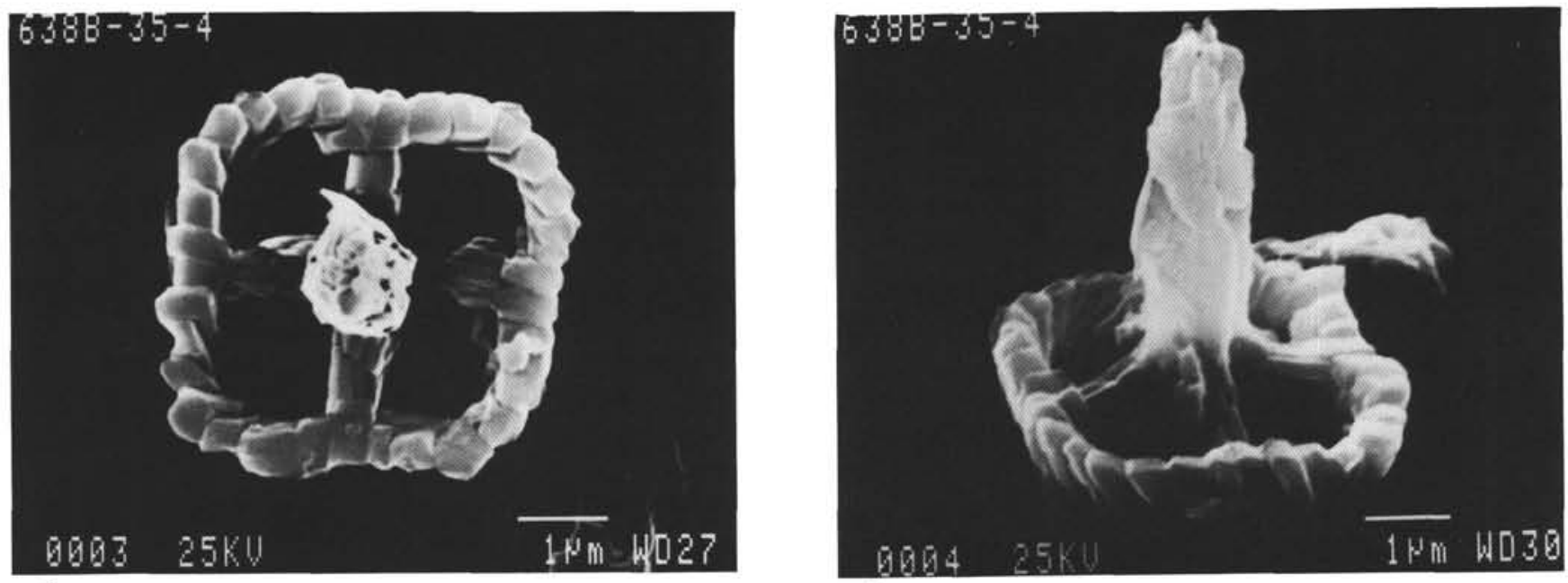

$$
1
$$

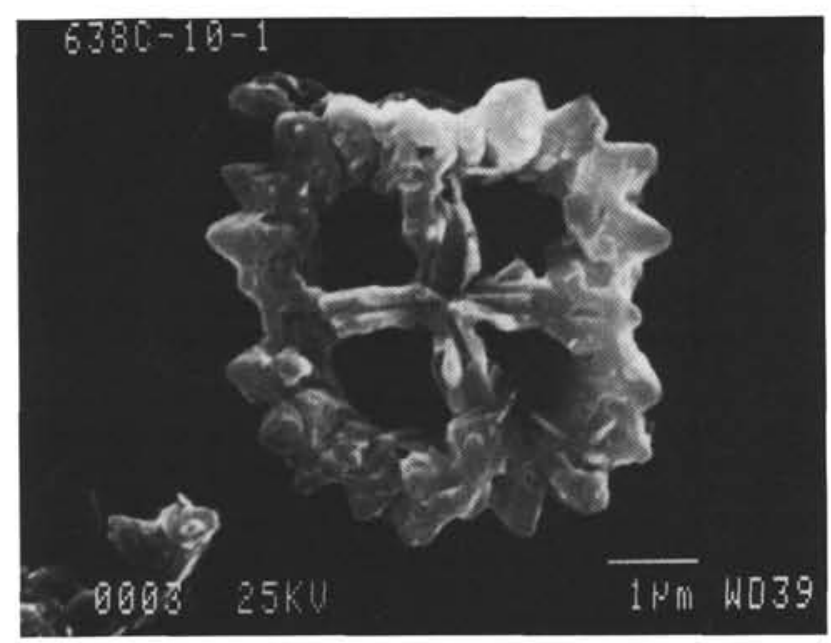

3

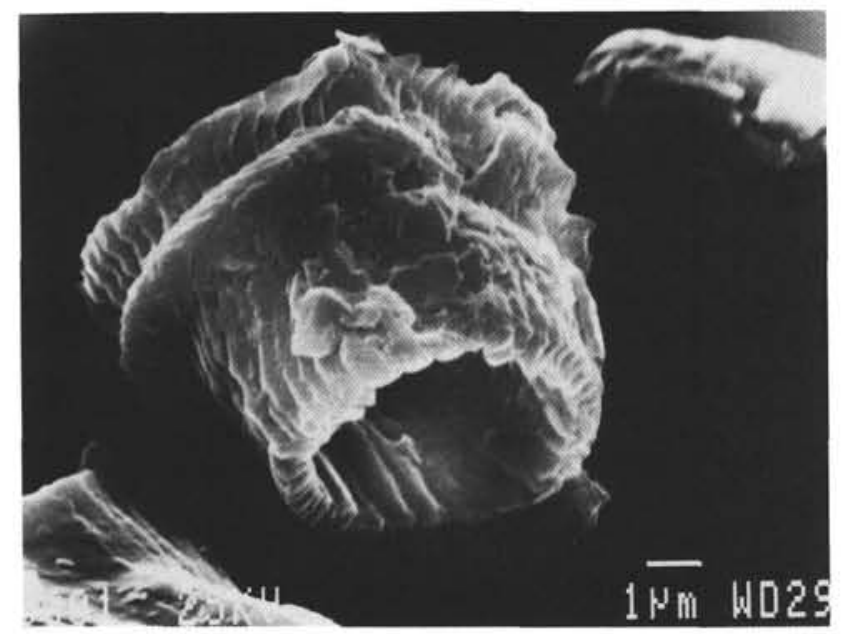

8



4

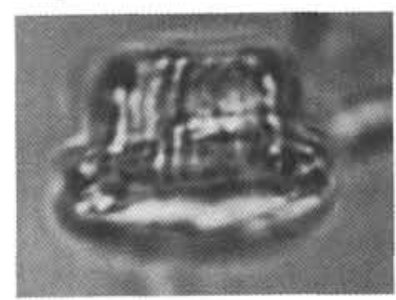

6

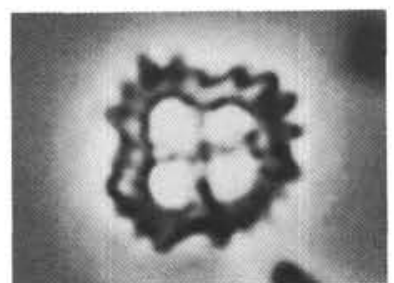

5

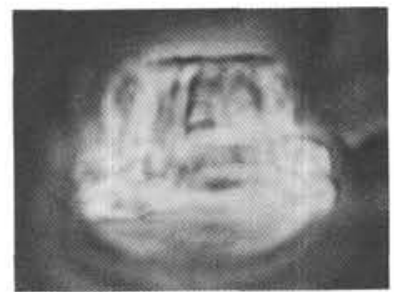

7

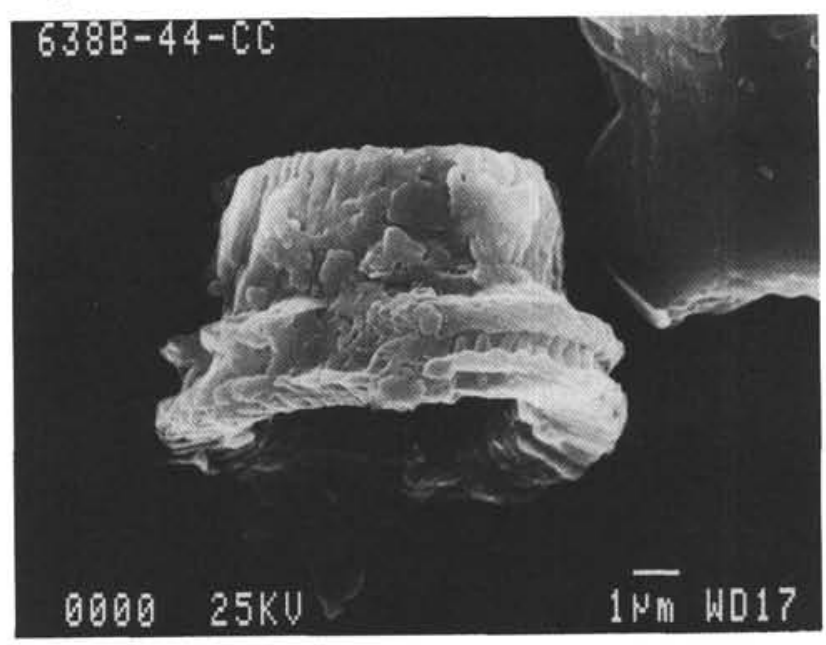

9

Plate 26. 1-5. Diadorhombus rectus Worsley (1 and 2) Sample 103-638B-35R-4, 34-35 cm, (1) D, 10,000 $\times$, (2) oblique D, 9700 $\times$, (3-5) Sample 103-638C-10R-1, 35-36 cm, (3) D, $9000 \times,(4-5)^{53} 3600 \times,(4, \mathrm{Pol} ; 5, \mathrm{Ph})$. 6-9. Tubodiscus verenae Thierstein, Sample 103-638B-44R, CC, (6 and $7)^{59} 2300 \times,(6, \mathrm{Tr} ; 7, \mathrm{Ph}),(8)$ oblique P, $5500 \times$, (9) L, $4800 \times$. 




1



3

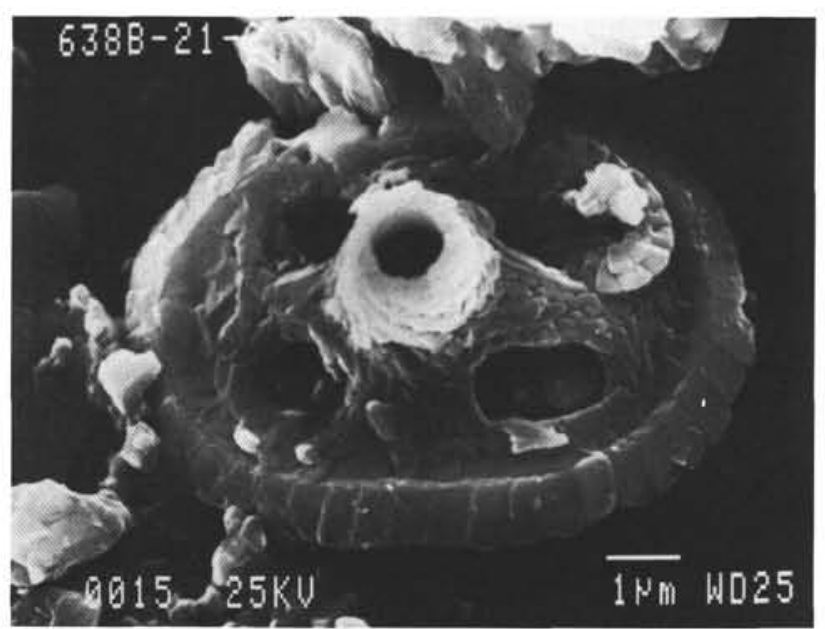

8

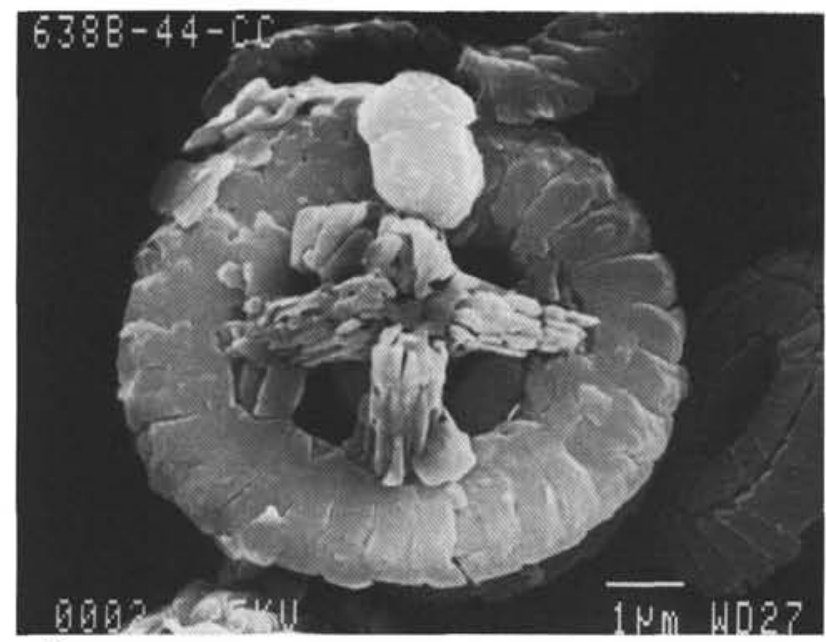

2

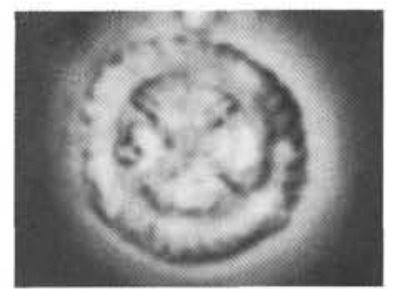

4

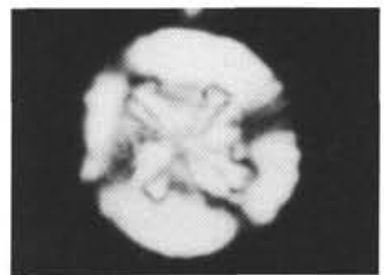

6

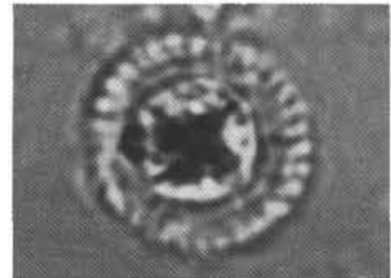

5

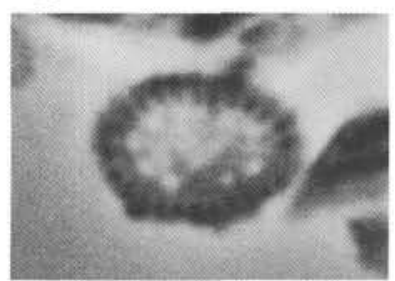

7

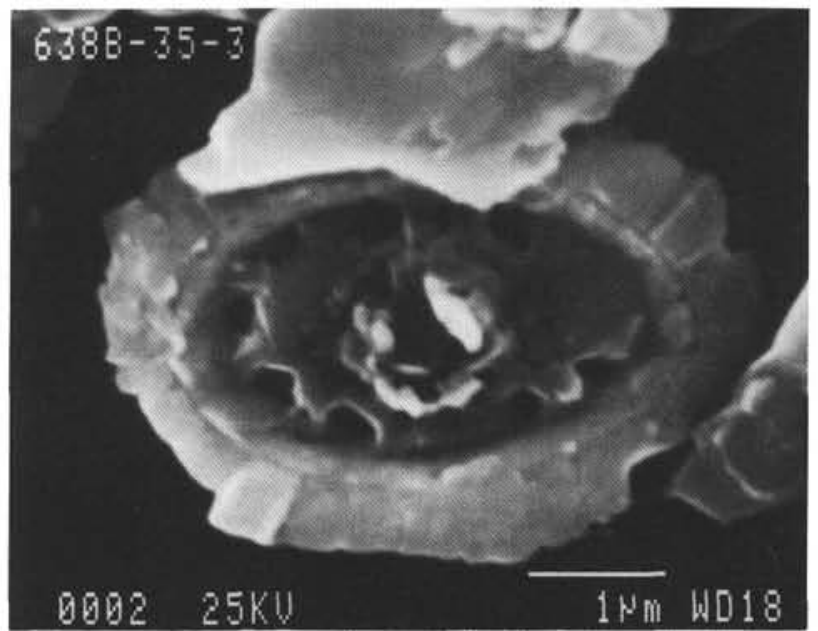

9

Plate 27. 1. Microstaurus chiastius (Worsley, Sample 103-638B-21R-3, 60-62 cm, D, 11,800×. 2-6. Microstaurus conus (Worsley), (2) Sample 103-638B-44R, CC, D, 8000×, (3-6) Sample 103-638B-35R-3, 111-112 cm (3) D, 6900 × , (4-6) ${ }^{\mathrm{s} 3} 3300 \times,(4, \mathrm{Ph} ; 5$, Tr; 6, Pol). 7 and 9. Perissocyclus noeliae (Black), Sample 103-638B-35R-3, 111-112 cm, (7) ${ }^{\mathrm{s} 9} 4500 \times, \mathrm{Ph},(9) \mathrm{D}, 14,000 \times$. 8. Axopodorhabdus dietzmannii (Reinhardt), Sample 103-638B-21R-2, 47-48 cm, D, 7300x. 


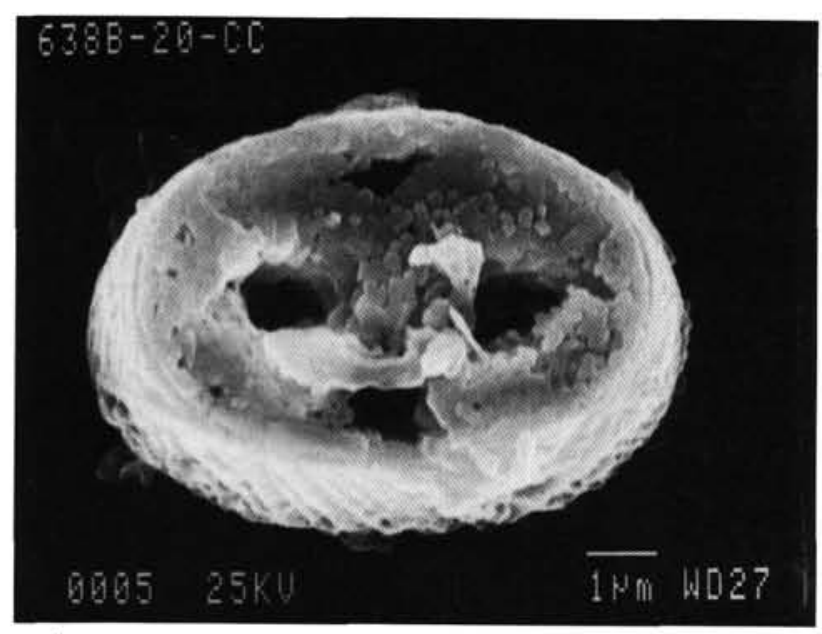

1

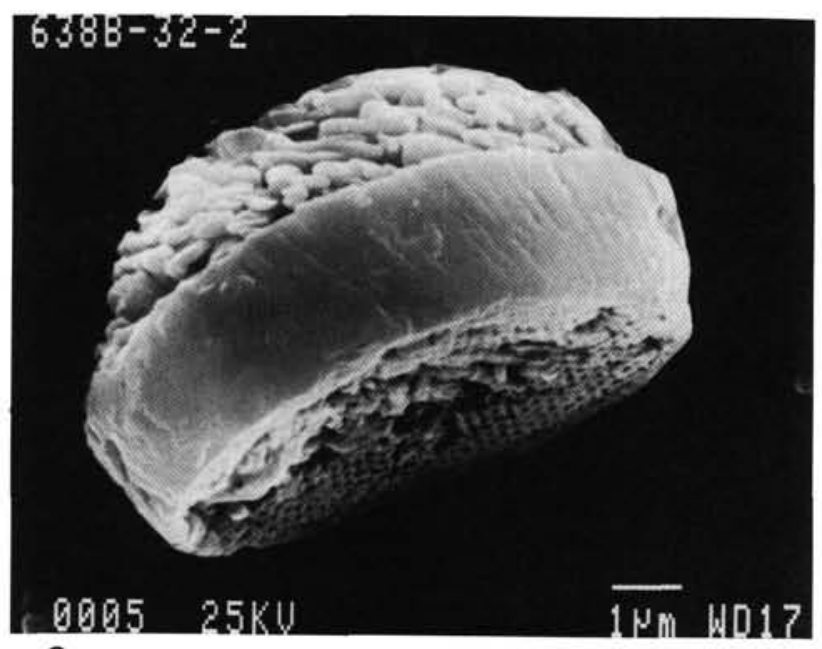
3

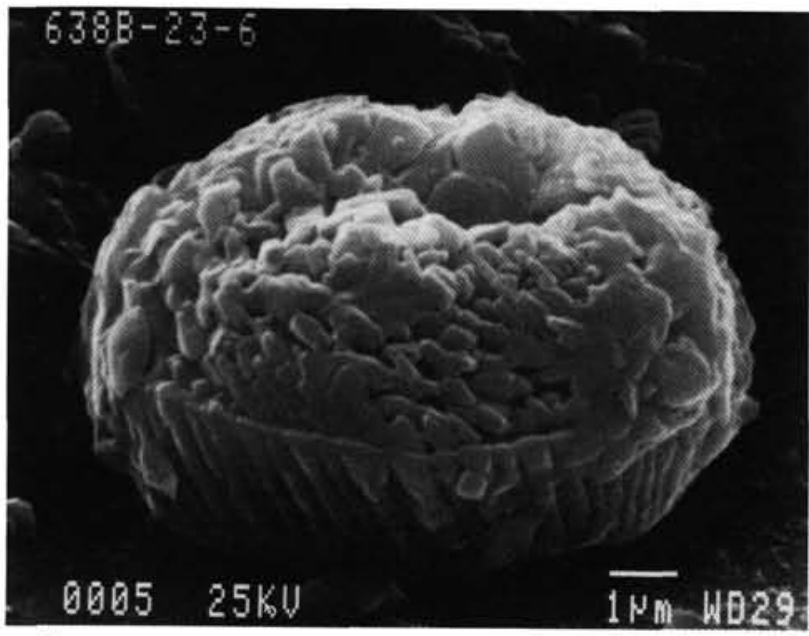
5



2

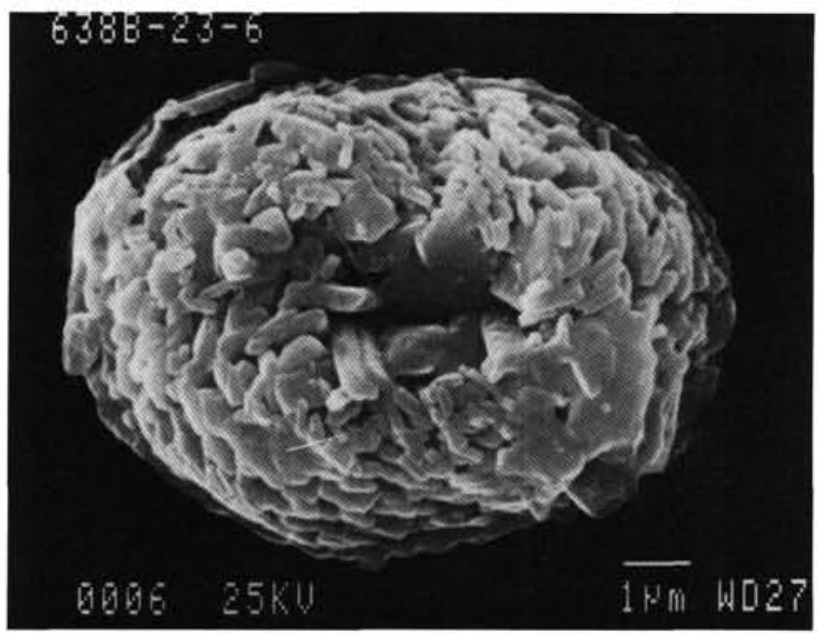

4



6

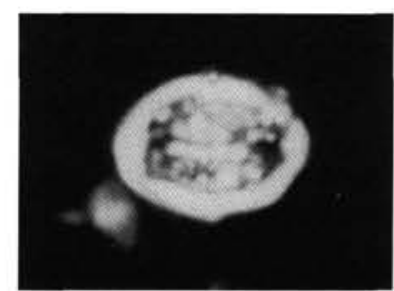

8

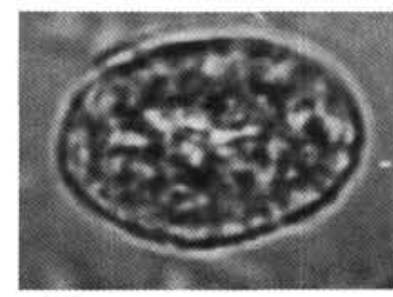

7

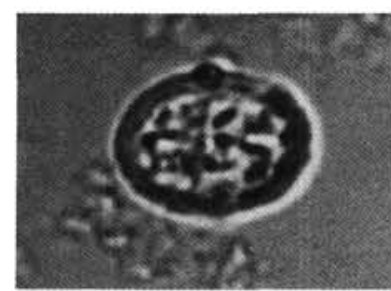

9

Plate 28. 1 and 2. Rhagodiscus infinitus (Worsley), Sample 103-638B-20R, CC, 7000 $\times$, (1, P; 2, D). 3-7. Calcicalathina oblongata (Worsley), (3) Sample 103-638B-32R-2, 8-9 cm. L, 7200 × , (4-7) Sample 103-638B-23R-6, 58-59 cm, (4), D, 6900 $\times$, (5) ${ }^{54}$ oblique D, $7000 \times,\left(6\right.$ and 7) ${ }^{\mathrm{s} 4} 3100 \times,(6$, Pol; 7, Tr). 8 and 9. Rhagodiscus cf. infinitus (Worsley), Sample 103-638B-10R-1, 35-36 cm, 2400×, (8, Pol; 9, Tr). 


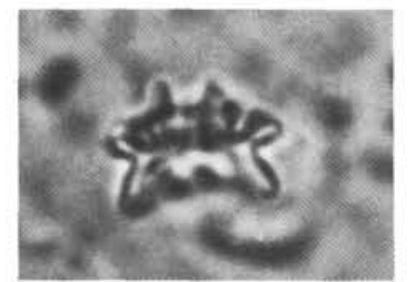

1

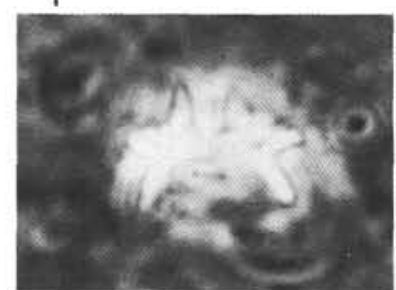

5

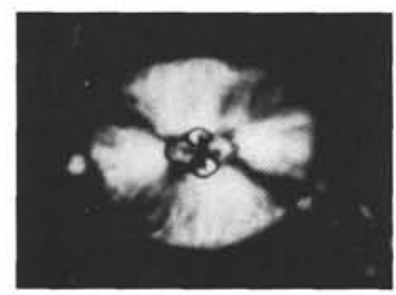

9

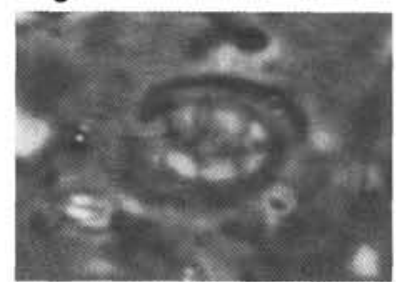

11

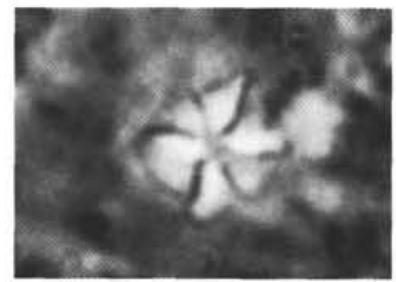

16



20

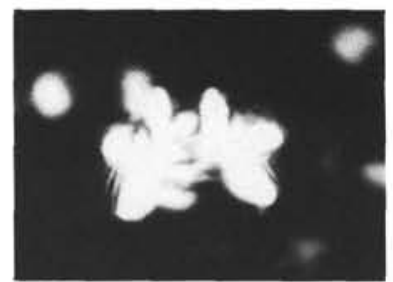

$$
2
$$

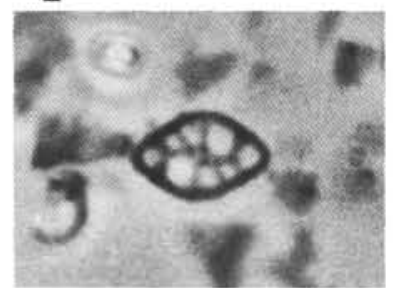

6

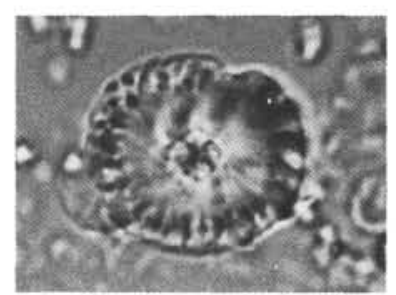

10

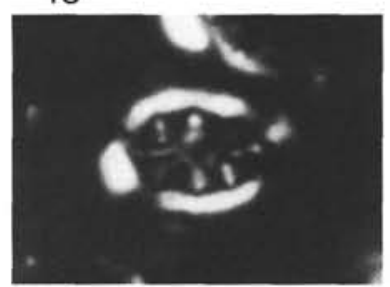

12



17

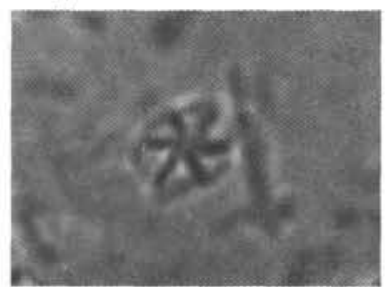

21

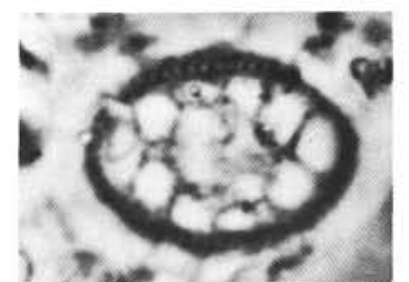

3

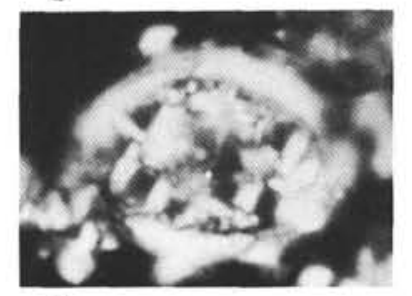

7



13

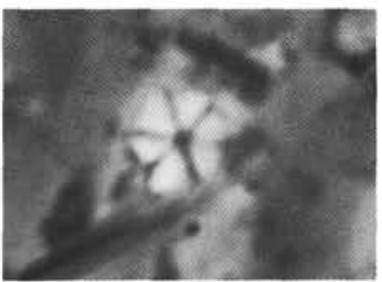

18

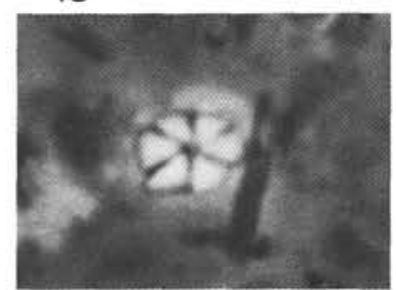

22

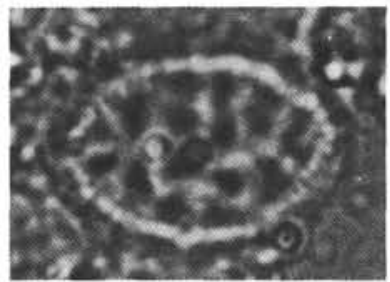

4

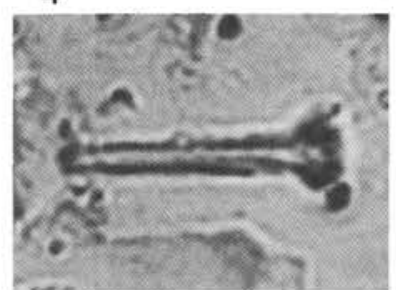

8



14

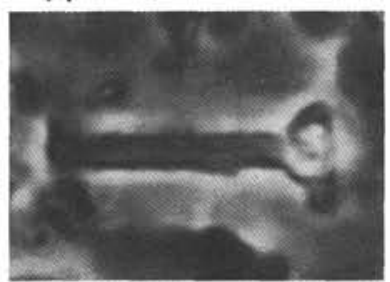

15

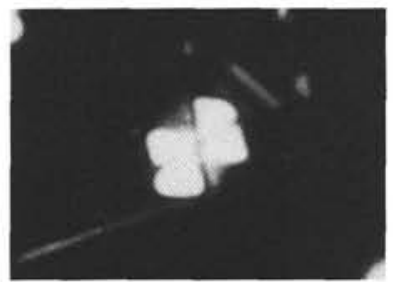

19

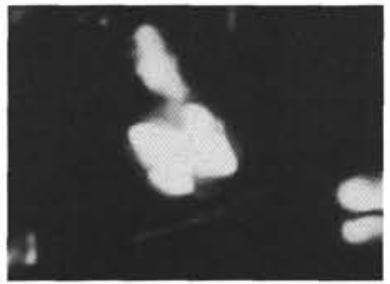

23

Plate 29. 1, 2, and 5. Darwinilithus pentarhethum Watkins, Sample 103-641A-6, CC (29 cm), 3700 x, (1, Tr; 2, Pol; 5, Ph). 3, 4, and 7. Octopodorhabdus plethotretus Wind and Cepek, Sample 103-638B-24R-3, 11-12 cm, 2800,$(3, \mathrm{Ph} ; 4, \mathrm{Tr} ; 7$, Pol). 6. Rhombolithion rhombicum (Stradner and Adamiker), Sample 103-638B-27R-5, 54-55 cm, 3800×, Tr. 8, 14, and 15. Vekshinella pseudocarinolithus Applegate and Bergen n. sp., Sample 103-638B-42R-2, 12-13 cm, 2700 $\times,(8, \mathrm{Tr} ; 14, \mathrm{Pol} ; 15, \mathrm{Ph}) .9$ and 10. Cruciellipsis cuvillieri ssp. grandis holotype, Applegate and Bergen n. ssp., Sample 103-638C-6R-1, 17-18 cm, 1800×, (9, Pol; 10, Tr). 11 and 12. Cretarhabdus sp. Sample 103-638B-44R-2, 115-116 cm 4100×, (11, $\mathrm{Tr} ; 12, \mathrm{Pol})$. 13. Micrantholithus sp., Sample 103-638B-25R-4, 48-49 cm, 1400 $\times$, Pol. 16-20. Rucinolithus wisei Thierstein, Sample 103-638B$44 \mathrm{R}-3,9-10 \mathrm{~cm},\left(16,17\right.$, and 20) $4400 \times,\left(16, \mathrm{Ph} ; 19^{\mathrm{si}}\right.$, Pol). 21-23. Rucinolithus cf. wisei Thierstein, Sample 103-638B-45R-3, 28-29 cm, 4500 $\times$, (21, $\mathrm{Tr} ; 22, \mathrm{Ph} ; 23, \mathrm{Pol})$. 